\title{
Lokale Algebren und Operatorprodukte am Punkt
}

\author{
Dissertation \\ zur Erlangung des Doktorgrades \\ der Mathematisch-Naturwissenschaftlichen Fakultäten \\ der Georg-August-Universität zu Göttingen
}

vorgelegt von

Henning Bostelmann

aus Soltau

Göttingen 2000 
D 7

Referent: Prof. Dr. D. Buchholz

Korreferent: Prof. Dr. H.-J. Borchers

Tag der mündlichen Prüfung: 1.11.2000 


\section{Inhaltsverzeichnis}

\begin{tabular}{llr}
\hline & Einleitung & 7
\end{tabular}

1.1 Punktfelder in der Quantenfeldtheorie . . . . . . . . . . . . . . . . . . . . . 8

1.2 Übersicht über die Arbeit . . . . . . . . . . . . . . . . . . . . . . . . 10

1.3 Technischer Hintergrund . . . . . . . . . . . . . . . . . . . . . . . . . . . . . . . . . . . .

1.3 .1 Algebraische Quantenfeldtheorie . . . . . . . . . . . . . . . . . . . . 12

1.3 .2 Wightman'sche Quantenfeldtheorie . . . . . . . . . . . . . . . . . . 14

\begin{tabular}{lr}
\hline Punktfelder & 17
\end{tabular}

2 Phasenraumstruktur $\quad 19$

2.1 Das Kurzabstandsverhalten von Quantenfeldtheorien . . . . . . . . . . . . 20

2.2 Asymptotische Phasenraumeigenschaften . . . . . . . . . . . . . . . . . . . . . . . . . . . . 23

2.3 Mathematische Formalisierung . . . . . . . . . . . . . . . . . . . . . . . . . 27

2.4 Ein Phasenraumkriterium . . . . . . . . . . . . . . . . . . . . . 30

2.A Garbenstrukturen . . . . . . . . . . . . . . . . . . . . 32

2.B Bilinearformen von endlichem Rang . . . . . . . . . . . . . . . . . . . . 36

2.C Abbildungen von asymptotisch endlichem Rang . . . . . . . . . . . . . . . 41

2.D Asymptotisches Verhalten . . . . . . . . . . . . . . . . . . . . . 45

3 Konstruktion von Punktfeldern 449

B.1 Feldinhalt . . . . . . . . . . . . . . . . . . . . . . . . . . . . . . . . . . . . . . . . . .

3.2 Analyse des $r$-Verhaltens . . . . . . . . . . . . . . . . . . . . . . 53

3.3 Felder als Grenzwerte lokaler Operatoren . . . . . . . . . . . . . . . . . . . 59

3.4 Lokalitätseigenschaften . . . . . . . . . . . . . . . . . . . 64

3.A Lokalisierte Zustände im Limes kleiner Abstände . . . . . . . . . . . . . . . 73

3.B Eine Erweiterung des Satzes von Hahn-Banach . . . . . . . . . . . . . . . 77

3.C Linearformen mit polynomialen Energieschranken . . . . . . . . . . . . . . 77

\begin{tabular}{llr}
\hline & Symmetrien & 81
\end{tabular}

4.1 Reformulierung der Struktur . . . . . . . . . . . . . . . . . . . . . . . . . . 82

4.2 Gruppendarstellungen . . . . . . . . . . . . . . . . . . . . . . . . . . . . . . . . . . . . . . . . .

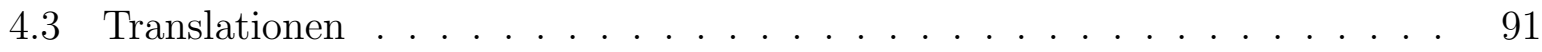

4.4 Differentiation der Felder . . . . . . . . . . . . . . . . . . . . . . . . 93

4. A Reguläre Projektionen in $\bar{\Sigma}^{*}$. . . . . . . . . . . . . . . . . . . . . . 97 
$\begin{array}{ll}\text { III Produkte von Feldern } & 99\end{array}$

5 Produktentwicklungen 101

5.1 Problemstellung . . . . . . . . . . . . . . . . . . . . . . . 102

5.2 Raumartige Produkte . . . . . . . . . . . . . . . . . . . . . . . . . . 103

5.3 Produktentwicklung für raumartige Abstände . . . . . . . . . . . . . . . . 109

5.4 Symmetrieeigenschaften der Approximationsterme . . . . . . . . . . . . . . 112

5.5 Produktentwicklung im Sinne von Distributionen . . . . . . . . . . . . . . 115

5. A Funktionentheoretische Ergänzungen . . . . . . . . . . . . . . . . . . . . 118

5.A.1 Ein Phragmén-Lindelöf-Argument . . . . . . . . . . . . . . . . . 118

5. A.2 Das Edge-ot-the-Wedge-Theorem . . . . . . . . . . . . . . . . . . 120

6 Normalprodukte 125

6.1 Das Konzept ${ }_{\text {NNormalprodukt }}{ }^{6}$. . . . . . . . . . . . . . . . . 126

6.2 Definition von Normalprodukten . . . . . . . . . . . . . . . . . . . . . . . . 127

6.3 bowenstein's rule . . . . . . . . . . . . . . . . . . . . . . . . . . . . . . . . . . . . . . . . . . . . . . . . . . . . . . . . . .

6.4 Feldgleichungen . . . . . . . . . . . . . . . . . . . . . 136

$\begin{array}{lll}\text { III Beispiele } & 141\end{array}$

7 Freie Feldtheorie $\quad 143$

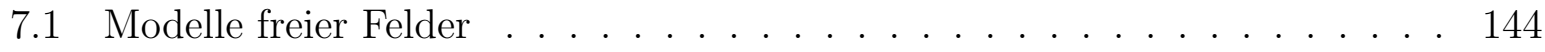

7.1 Einteilchenraum . . . . . . . . . . . . . . . . . . . . 144

7.1 .2 Fockraum . . . . . . . . . . . . . . . . . . . . 145

7.1 .3 Lokale Algebren . . . . . . . . . . . . . . . . . . . . . . . . 146

7.2 Nachweis des Phasenraumkriteriums . . . . . . . . . . . . . . . . . . 146

7.2 .1 Vorgehensweise . . . . . . . . . . . . . . . . . . . . . . 147

7.2 .2 Entwicklung nach skalenunabhängigen Funktionen . . . . . . . . . . 148

7.2 .3 Entwicklung nach skalenabhängigen Funktionen . . . . . . . . . . . 152

7.2 .4 Autspaltung der Weyloperatoren . . . . . . . . . . . . . . . . . 153

7.2 .5 Erweiterung und Normkonvergenz der Reihe . . . . . . . . . . . . . 155

$7.2 .6 \quad$ Anwendung auf die Abbildung $\exists$. . . . . . . . . . . . . . . . . . 159

7.2.A Multiindex-Schreibweise . . . . . . . . . . . . . . . . . . . 161

7.2.B Spezielle Funktionale . . . . . . . . . . . . . . . . . . . . . . 162

7.2.C Spurnorm der Operatoren $T^{ \pm}(E, r)$ für das reelle skalare freie Feld . 166

7.2.D Die kleinste obere Schranke zweier Operatoren . . . . . . . . . . . . 169

7.3 Bestimmung des Feldinhalts . . . . . . . . . . . . . . . . . . . . . . . . . . . . . . . . .

7.3 .1 Vorgehensweise . . . . . . . . . . . . . . . . . 172

7.3 .2 Funktionen im Einteilchenraum . . . . . . . . . . . . . . . . . . 174

7.3 .3 Verallgemeinertes Gram-Schmidt-Verfahren . . . . . . . . . . . . . . 177

7.3 .4 Zweite Quantisierung . . . . . . . . . . . . . . . . . . . 180

7.3. A Ein Hilfssatz im Einteilchenraum . . . . . . . . . . . . . . . . . . . 183

7.4 Freie Wightman-Felder . . . . . . . . . . . . . . . . . . . . . . . . . . . . . 184

7.4 .1 Das reelle skalare Feld im Wightman-Rahmen . . . . . . . . . . . . 184

7.4 .2 Rekonstruktion des Feldes . . . . . . . . . . . . . . . . . . . . . . . 186 
7.5 Produktentwicklung und Normalproduktraum . . . . . . . . . . . . . . . . 189

8 Erweiterungen und Ausblick 193

8.1 Weitere Aspekte der treien Theorie . . . . . . . . . . . . . . . . . . . . . . 194

8.2 Wechselwirkung, Eichtheorien . . . . . . . . . . . . . . . . . . . . . . . . 197

\begin{tabular}{ll}
\hline Notationskonventionen & 201
\end{tabular}

$\begin{array}{ll}\text { Literaturverzeichnis } & 205\end{array}$ 



\section{Kapitel 1}

\section{Einleitung}

Bei der Formulierung von Modellen in der relativistischen Quantenphysik spielen punktartig lokalisierte Meßgrößen (Quantenfelder) eine zentrale Rolle. Sie bilden die begriffliche Grundlage für Feldgleichungen oder Wirkungsprinzipien, mit deren Hilfe sich spezielle Modelle auszeichnen und störungstheoretisch behandeln lassen.

Die scharfe Lokalisierung der Quantenfelder im Ortsraum führt aufgrund der quantenmechanischen Unschärferelation jedoch zu Singularitäten, die eine mathematisch rigorose Formulierung erschweren. Insbesondere sind Produkte solcher Felder am gleichen RaumZeit-Punkt im allgemeinen nicht wohldefiniert, sondern weisen Divergenzen auf. Dies führt zu Problemen etwa bei der Behandlung nichtlinearer Feldgleichungen, von denen man erwartet, daß sie die Dynamik wechselwirkender Modelle bestimmen.

Die vorliegende Arbeit stellt eine neue Methode zur modellunabhängigen Analyse der Eigenschaften von Punktfeldern vor. Ausgehend von einer Formulierung der Theorie durch Meßgrößen, die in endlichen Raum-Zeit-Gebieten lokalisiert sind (und damit keine Singularitäten aufweisen), untersuchen wir deren Kurzabstandsverhalten. Wir geben ein natürliches Phasenraumkriterium an, das uns erlaubt, den Feldinhalt der Theorie zu bestimmen, indem wir Punktfelder als Limiten immer besser lokalisierter Observablen konstruieren. Deren singuläres Verhalten wird analysiert.

Dasselbe Phasenraumkriterium ermöglicht auch die Behandlung von Produkten der konstruierten Felder: Im Limes kleiner Abstände kann eine asymptotische Reihenentwicklung der Feldprodukte (Operatorproduktentwicklung) etabliert werden. Diese gestattet die Untersuchung von Normalprodukten und damit die Formulierung nichtlinearer Feldgleichungen im vorliegenden Rahmen.

Das genannte modellübergreifende Kriterium wird beispielhaft in Modellen der freien Feldtheorie explizit hergeleitet und untersucht; die Ergebnisse lassen Extrapolationen auch auf den wechselwirkenden Fall zu. 


\subsection{Punktfelder in der Quantenfeldtheorie}

Der historisch älteste und auch heute noch am weitesten verbreitete Zugang zur Quantenfeldtheorie führt über punktartig lokalisierte Quantenfelder, die diesem Gebiet letztlich auch den Namen gaben. Diese Punktfelder $\phi(x)$ beschreiben im einfachsten Fall (soweit es sich um neutrale Bose-Felder handelt) physikalische Messungen, die an einem Raum-ZeitPunkt $x$ lokalisiert sind. Alle anderen Observablen der Theorie werden aus Funktionen (Integralen, Ableitungen) dieser Felder aufgebaut. Symbolisch wird eine Quantenfeldtheorie also charakterisiert durch eine Zuordnung

$$
x \mapsto\{\phi(x)\},
$$

wobei die Menge $\{\phi(x)\}$ alle am Punkt $x$ lokalisierten Observablen enthält, also auch Ströme, Energiedichten u.ä.

Die Idealisierung einer punktartig lokalisierten Messung ergibt sich zwar in natürlicher Weise, wenn man zur Quantenfeldtheorie durch „Quantisierung“ einer klassischen Theorie gelangt; sie führt jedoch zu Problemen bei der mathematischen Formulierung. Es stellt sich heraus, daß die Punktfelder sehr singuläre Objekte sind; mathematisch handelt es sich um unbeschränkte quadratische Formen, was physikalisch bedeutet, daß der Erwartungswert der zugehörigen Messung nicht in allen denkbaren Zuständen des Systems angegeben werden kann. Man kann dies als Auswirkung der Unschärferelation deuten: Eine im Ortsraum beliebig gut lokalisierte Messung muß einen beliebig großen Energie-Impuls-Übertrag aufweisen, d.h. ihr Hochenergieverhalten wird singulär.

Dies macht nicht nur eine mathematisch stringente Beschreibung technisch aufwendig, sondern führt spätestens dann zu manifesten Problemen, wenn Produkte dieser Felder am selben Raum-Zeit-Punkt $x$ definiert werden sollen, wie es z.B. zur Formulierung von Wechselwirkungstermen in Lagrangefunktionen notwendig ist. Auch die Behandlung nichtlinearer Feldgleichungen wird hierdurch sehr erschwert.]

Zur axiomatischen Formulierung von Feldtheorien wurden daher andere Methoden als die der punktartig lokalisierter Felder entwickelt. Man hob die scharfe Lokalisierung auf, indem die Felder mit Testfunktionen $f$, verschmiert" wurden, so daß man Operatoren $\phi(f)$ erhält (Wightman-Distributionen). Eine Theorie wird in diesem Bild also grob gesprochen durch eine Zuordnung

$$
f \mapsto\{\phi(f)\}
$$

beschrieben. Die Operatoren $\phi(f)$ sind zwar im allgemeinen unbeschränkt, aber dennoch "regulär" genug, um ein Produkt zwischen ihnen definieren zu können. Dieser Zugang [SW64] ermöglichte es, gewisse Eigenschaften von Quantenfeldtheorien, wie das PCTTheorem oder den Spin-Statistik-Zusammenhang, auf allgemeine Axiome zurückzuführen.

Später löste man sich ganz vom Begriff der Quantenfelder und ging im Rahmen der sogenannten algebraischen Formulierung zur Betrachtung allgemeiner Observablen über, die in endlichen Raum-Zeit-Gebieten $\mathcal{O}$ lokalisiert sind [HK64]; zu jedem solchen Gebiet erhält man eine von den Observablen erzeugte Algebra $\mathfrak{A}(\mathcal{O})$ :

$$
\mathcal{O} \mapsto \mathfrak{A}(\mathcal{O})
$$

\footnotetext{
1 "This problem has plagued quantum field theory throughout its history." [Haa.96, p. 45]
} 
Die Elemente der Algebren sind hier beschränkte Operatoren, so daß die algebraischen Relationen (insbesondere die Multiplikation) zwischen ihnen wohldefiniert sind. Konzeptionell wichtiger als dieser technische Punkt ist aber, daß man die „Koordinatisierung“ der Observablen durch Felder aufgibt: Als relevant erweist sich nur ihre Lokalisierung in der Raum-Zeit und die „abstrakte“ algebraische Struktur.] Dieser Formalismus hat sich zur modellunabhängige Analyse von Quantenfeldtheorien sehr bewährt, etwa für Untersuchungen zu Superauswahlsektoren und Austauschstatistik [DHR74, BF87], zur Teilcheninterpretation [BPS91, Por99], zur Renormierungsanalyse [BV95] und zu thermodynamischen Aspekten [BW86, B.18.9].

Allerdings ist im Rahmen der lokalen Algebren die Punktfeldstruktur der Theorie nicht a priori sichtbar. Zwar existieren Ergebnisse zum Punktfeldinhalt solcher Theorien [FH81], doch hat man über die Struktur dieser Felder relativ wenig detaillierte Aussagen, und insbesondere der Aspekt der nichtlinearen Feldgleichungen hat keine direkte Entsprechung. Aus diesem Grund gibt es bisher kaum Methoden zur Auszeichnung konkreter Theorien im algebraischen Rahmen [Haa.93].

Die Analyse von Punktfeldern und ihren Produkten bewegt sich insofern bislang meist im störungstheoretischen Kontext oder im Rahmen exakt lösbarer zweidimensionaler Modelle. Eine Möglichkeit zur Beschreibung der bei der Produktbildung auftretenden Divergenzen bieten sogenannte Operatorproduktentwicklungen [Wil69], das sind asymptotische Reihenentwicklungen von Punktfeld-Produkten der Art

$$
\phi(x) \cdot \phi^{\prime}(y)=\sum_{j} c_{j}(x-y) \phi_{j}\left(\frac{x+y}{2}\right)
$$

mit $c$-Zahl-Funktionen $c_{j}(x)$ und Punktfeldern $\phi_{j}(x)$, die im Limes kleiner $x-y$ gültig sind. Solche Entwicklungen wurden zunächst störungstheoretisch eingeführt [Zim70] und spielen in der Lagrange'schen Feldtheorie eine wichtige Rolle [Wei96, ch. 20]. Axiomatische Ansätze im Rahmen der Wightman-Theorie wurden zwar untersucht [WZ72, SSe73], aber eine Herleitung „from first principles“ konnte nie ganz erreicht werden: Die Existenz der Reihe scheint nicht aus den Wightman-Axiomen zu folgen; sie läßt sich unter der zusätzlichen Annahme einer konformen Symmetrie etablieren [SSV75], doch ist unklar, welche physikalischen Eigenschaften im allgemeinen Fall eine wohldefinierte Operatorproduktentwicklung nach sich ziehen.

Solche Produktentwicklungen kann man nun auswerten, um einen „endlichen Anteil“ der im allgemeinen für $x \rightarrow y$ divergenten Approximationsterme $c_{j}(x-y) \phi_{j}\left(\frac{x+y}{2}\right)$ auszuzeichnen und diesen als Normalprodukt der Felder zu verwenden, in Verallgemeinerung des Wickprodukts der freien Feldtheorie. Dieses Normalprodukt kann dann z.B. zur Formulierung nichtlinearer (wechselwirkender) Feldgleichungen dienen [Zim67, Low70]. Diese Ergebnisse sind jedoch eng an die Struktur bestimmter Modelle gebunden und bisher kaum systematisch (unabhängig von konkreten Modellen oder Zusatzannahmen) analysiert worden.

\footnotetext{
${ }^{2}$ Genauer ist der physikalische Inhalt der Theorie nicht in den Algebren bei festem $\mathcal{O}$ codiert - sie sind unter plausiblen Voraussetzungen an die Phasenraumstruktur alle isomorph [BDF87] -, sondern in der Inklusionsstruktur $\mathfrak{A}\left(\mathcal{O}_{1}\right) \subset \mathfrak{A}\left(\mathcal{O}_{2}\right)$ für $\mathcal{O}_{1} \subset \mathcal{O}_{2}$.
} 


\section{2 Übersicht über die Arbeit}

Das Anliegen dieser Arbeit ist eine modellunabhängige, mathematisch rigorose Analyse der Eigenschaften von Punktfeldern und ihrer Produkte. Hierzu gehen wir von einem qualitativen Bild des Verhaltens einer Quantenfeldtheorie bei kleinen Phasenraumvolumina aus. Auf dieser Grundlage bestimmen wir den Feldinhalt der Theorie und untersuchen das singuläre Verhalten der Punktfelder. Weiter etablieren wir eine Operatorproduktentwicklung, betrachten Normalprodukte und Feldgleichungen zwischen den konstruierten Größen.

Als mathematische Grundlage für eine solche Analyse eignet sich der oben schon erwähnte Rahmen der algebraischen Feldtheorie..$^{3}$ Wir gehen aus von einem Netz $\mathcal{O} \mapsto \mathfrak{A}(\mathcal{O})$, das von beschränkten und in endlich großen Gebieten $\mathcal{O}$ lokalisierten Observablen erzeugt wird. Dieser Rahmen ist weit allgemeiner als der einer Quantenfeldtheorie aus Punktfeldern - er könnte auch nicht-punktartig lokalisierte Objekte beschreiben, wie etwa MandelstamStrings oder Wilson-Loops. Wir analysieren hier jedoch das Kurzabstandsverhalten solch einer Theorie und untersuchen insbesondere, ob sich im Limes kleiner Gebiete $\mathcal{O}$ lokale Punktfelder aus den Algebren konstruieren lassen.

Teil I der Arbeit beschäftigt sich mit der Konstruktion von Punktfeldern in diesem Rahmen.

Hierzu formulieren wir ein natürliches Phasenraumkriterium, das in Kapitel 2 motiviert und mathematisch präzisiert wird. Es beruht auf der heuristischen Vorstellung, daß sich bei gleichzeitiger Einschränkung im Orts- und Impulsraum die Struktur der Theorie sehr gut durch nur endlich viele unabhängige Meßgrößen beschreiben läßt.

Genauer betrachten wir folgende Situation: Die Auswertung lokaler Observablen $A$ auf energiebeschränkten Zuständen $\sigma$, in Formeln

$$
(\sigma, A) \mapsto \sigma(A)
$$

sollte sich im Limes kleiner Lokalisationsgebiete und nicht zu großer Energien durch Approximation mit endlich vielen „Standardobservablen“ $\phi_{j}$ und „Standardzuständen“ $\sigma_{j}$ beschreiben lassen:

$$
\sigma(A) \approx \sum_{j} \sigma\left(\phi_{j}\right) \sigma_{j}(A)
$$

Die $\phi_{j}$ sind dann Kandidaten für lokale Punktfelder. In der Tat erlaubt es das präzise formulierte Phasenraumkriterium, eine Folge von eindeutig definierten endlichdimensionalen Räumen zu konstruieren, deren Elemente sich als am Punkt $x=0$ lokalisierte Wightman-Felder herausstellen. Die Felder sind nach der Stärke ihrer Singularität (ihrer „Dimension“) geordnet. Der berechnete Feldinhalt stimmt mit dem in der Literatur bekannten [FH81] überein. Diese Analyse findet sich in Kapitel 3 .

Die konstruierten Punktfelder „erben“ eine Reihe von Eigenschaften von der zugrundeliegenden algebraischen Theorie; insbesondere sind sie z.B. kovariant unter Lorentztransformationen und Translationen. Außerdem ist der Feldinhalt abgeschlossen unter Differentiation. Diese Aspekte werden in Kapitel 4 in allgemeinerem Rahmen untersucht.

Teil II der Arbeit beschäftigt sich dann mit der Untersuchung von Produktstrukturen zwischen den konstruierten Feldern.

\footnotetext{
${ }^{3}$ Eine genaue Auflistung des zugehörigen Axiomensystems findet man in Abschnitt 1.3.1.
} 
In Kapitel 5 etablieren wir in unserem Rahmen eine rigorose Version der Operatorproduktentwicklung (1.1.4). Wir verwenden dazu erneut das Phasenraumkriterium. Die Ergebnisse gestatten eine weitere Untersuchung der Koeffizientenfunktionen $c_{j}(x)$, die trotz des modellunabhängigen Ansatzes sehr direkt bekannt sind. Im Fall raumartig getrennter Argumente der multiplizierten Felder sind die Koeffizienten holomorph fortsetzbare Funktionen, deren Divergenzgrad im Limes koinzidierender Punkte abgeschätzt werden kann. Für beliebige Abstände der Felder existieren die Koeffizienten noch als Distributionen. Wir untersuchen außerdem die Kovarianzeigenschaften der $c_{j}(x)$ unter anderem unter Lorentztransformationen und inneren Symmetrien.

Die Operatorproduktentwicklung erlaubt die Definition und Analyse von Normalprodukten, die als Verallgemeinerungen des Wick-Produkts der freien Theorie verstanden werden können (Kapitel 6). Wir leiten damit eine nicht-perturbative Fassung von Ergebnissen her, die in der Literatur in speziellen Modellen und mit störungstheoretischen Methoden gewonnen wurden. Hierbei wird deutlich, daß ein Normalprodukt von Feldern sich im allgemeinen nicht eindeutig definieren läßt. Vielmehr kann ein gewisser endlichdimensionaler Vektorraum als „Spur der Produktentwicklung“ ausgezeichnet werden; die konkrete Definition des Normalprodukts entspricht einer Auswahl von Elementen in diesen Raum. Die Normalprodukte erlauben uns auch die Betrachtung nichtlinearer Feldgleichungen: Wir bestimmen den „Feldgleichungsinhalt" einer gegebenen Theorie.

In Teil III der Arbeit diskutieren wir die Anwendung des entwickelten (modellübergreifenden) Formalismus auf konkrete Modelle. Dazu überprüfen wir zunächst in Kapitel Ø das untersuchte Phasenraumkriterium in der Theorie eines freien reellen skalaren Teilchens. Abgesehen von Infrarotdivergenzen bei niederdimensionalen Systemen $(d \leq 2+1)$ lassen sich die untersuchten Strukturen hier explizit etablieren; man erhält durch unsere Konstruktion die bekannten Punktfelder der Theorie (inklusive Ableitungen und Wickprodukte) zurück.

Kapitel 8 diskutiert dann Erweiterungen auf allgemeinere freie Theorien sowie heuristische Extrapolationen auf wechselwirkende Theorien (deren rigorose Konstruktion in physikalischer Raumzeit bisher nicht gelungen ist). Wir skizzieren ein Kriterium zur Charakterisierung von Eichtheorien anhand ihres Punktfeldinhalts.

Auf diese Weise erhalten wir, ausgehend von einem sehr allgemeinen (dem algebraischen) Rahmen, nicht-perturbative und modellunabhängige Aussagen über das Kurzabstandsverhalten von Quantenfeldtheorien, was sich explizit im Verhalten von Punktfeldern und ihren Produkten wiederspiegelt. Betrachtet man das genannte Phasenraumkriterium als plausible physikalische Annahme, dann sollten unsere Ergebnisse - wie etwa die Existenz der Operatorproduktentwicklung - in allen physikalisch realistischen Theorien gültig sein.

Abstrakter zeichnen wir durch Angabe des Phasenraumkriteriums eine bestimmte Klasse von Wightman-Theorien mit besonders angenehmen Eigenschaften aus (unter anderem einem „regulären“ Verhalten der Feldprodukte im Limes scharfer Lokalisierung). Insofern ist die Arbeit auch ein Beitrag zum Verständnis der Feldtheorie im Wightman'schen Formalismus.

Umgekehrt könnte die vorliegende Analyse zu einem besseren Verständnis des algebraischen Rahmens führen. Bisher werden konkrete Modelle der Feldtheorie fast ausschließlich im Kontext von Punktfeldern konstruiert; im Rahmen der algebraischen Theorie ist dies nur schwer möglich, da die gesamte heuristische Information über feldtheoretische Modelle in Form von Feldgleichungen oder Lagrangefunktionen, d.h. als Relationen zwischen punkt- 
artig lokalisierten Größen, vorliegt. Das Wissen um die Einbettung der Punktfelder in den algebraischen Kontext sollte auch hier neue Erkenntnisse ermöglichen.

Von einem mehr mathematischen Standpunkt gesehen, leistet die vorliegende Arbeit eine Klassifikation von lokalen Netzen (Präkogarben) aus von-Neumann-Algebren. Unter Zuhilfenahme einer einparametrigen Symmetriegruppe mit positivem Generator zeichnen wir eine gewisse Klasse solcher Präkogarben aus und konstruieren dort eine Folge von endlichdimensionalen Vektorräumen, die Invarianten des Netzes sind. Die Elemente der Vektorräume sind, als operatorwertige Distributionen aufgefaßt, affiliiert zum lokalen Netz; die Räume selbst sind abgeschlossen unter Symmetrietransformationen, Differentiation und in gewissem Sinne unter Produktbildung.

Die Arbeit baut auf Ergebnissen aus [Bos98] auf bzw. übernimmt diese zum Teil in leicht verändertem Kontext.

\subsection{Technischer Hintergrund}

Wir führen unsere Analyse zunächst im Rahmen der algebraischen Quantenfeldtheorie durch, und zwar im Vakuumsektor. Die hier verwendeten Axiome sind in Abschnitt 1.3.1 aufgeführt. Für einige Teilbereiche (insbesondere für die präzise Definition des PunktfeldBegriffs) beziehen wir uns auf die Wightman-Axiome, die in Abschnitt 1.3.2 zusammengestellt sind.

Die Relationen zwischen den beiden Formalismen werden in den Kapiteln 2 und 3 ausführlich diskutiert.

\subsubsection{Algebraische Quantenfeldtheorie}

Der algebraische Zugang zur Quantenfeldtheorie („Local Quantum Physics“ [Haa.96]) verwendet als Grundobjekt ein Netz von Algebren, d.h. eine Abbildung

$$
\mathcal{O} \mapsto \mathfrak{A}(\mathcal{O}),
$$

die jeder offenen Teilmenge $\mathcal{O}$ des Minkowskiraums $\mathcal{M}:=\mathbb{R}^{s+1}$ eine $C^{*}$-Algebra $\mathfrak{A}(\mathcal{O})$ zuordnet; dabei fordert man Isotonie:

$$
\mathfrak{A}\left(\mathcal{O}_{1}\right) \subset \mathfrak{A}\left(\mathcal{O}_{2}\right) \quad \text { für } \mathcal{O}_{1} \subset \mathcal{O}_{2} .
$$

Physikalisch stehen die lokalen Algebren $\mathfrak{A}(\mathcal{O})$ - respektive ihre selbstadjungierten Elemente - für die im Gebiet $\mathcal{O}$ meßbaren Observablen. Deren relativistische Lokalisierung läßt sich dann durch die algebraischen Relationen beschreiben: Sind $\mathcal{O}_{1}$ und $\mathcal{O}_{2}$ raumartig getrennt, dann stellt man die Lokalitätsbedingung

$$
\left[A_{1}, A_{2}\right]=0 \quad \text { für } A_{i} \in \mathfrak{A}\left(\mathcal{O}_{i}\right) .
$$

Weiterhin ist eine Darstellung $\alpha(x, \Lambda)$ der Poincaré-Gruppef $\mathfrak{P}$ durch Automorphismen des Netzes $\mathfrak{A}(\mathcal{O})$ gegeben, die geometrisch auf den lokalen Algebren wirkt:

$$
\alpha(x, \Lambda) \mathfrak{A}(\mathcal{O})=\mathfrak{A}(\Lambda \mathcal{O}+x) .
$$

\footnotetext{
${ }^{4}$ Präziser ist hier und im folgenden mit $\mathfrak{P}$ die Zusammenhangskomponente der $\mathbf{1}$ in der Poincaré-Gruppe gemeint (sonst oft mit $\mathfrak{P}_{+}^{\uparrow}$ bezeichnet). Analog ist $\mathfrak{L}$ die 1 -Zusammenhangskomponente der Lorentzgruppe.
} 
Die vorliegende Arbeit beschränkt sich auf die Analyse des Vakuumsektors eines solchen Netzes: Wir nehmen die $\mathfrak{A}(\mathcal{O})$ als schwach abgeschlossene Unteralgebren von $\mathfrak{B}(\mathcal{H})$ für einen Hilbertraum $\mathcal{H}$ an, also als von-Neumann-Algebren. Weiter sei die Darstellung $\alpha$ unitär implementiert, d.h. sie rühre von einer unitären Darstellung $U(x, \Lambda)$ von $\mathfrak{P}$ auf $\mathcal{H}$ her:

$$
\alpha(x, \Lambda)=U(x, \Lambda) \cdot U(x, \Lambda)^{*} .
$$

Wir verlangen, daß $U(x, \Lambda)$ bezüglich der starken Operatortopologie stetig in $x, \Lambda$ ist. Dann kann $U(x, \Lambda)$ als Exponentialfunktion seiner selbstadjungierten Generatoren geschrieben werden; man hat etwa für die Translationen

$$
U(x, \mathbf{1}) \equiv U(x)=e^{i P_{\mu} x^{\mu}} .
$$

Die unbeschränkten selbstadjungierten Operatoren $P_{\mu}(\mu=0 \ldots s)$ werden als EnergieImpuls-Operatoren interpretiert; speziell ist $H=P_{0}$ der Hamilton-Operator. Seine Spektralprojektoren auf das Intervall $[0, E]$ bezeichnen wir mit $P(E)$. Die Darstellung $U$ soll die Spektrumsbedingung erfüllen, d.h. das gemeinsame Spektrum der kommutierenden Operatoren $P_{\mu}$ liege im abgeschlossenen Vorwärtslichtkegel $\bar{V}_{+}$(„Positivität der Energie“). Schließlich gebe es in $\mathcal{H}$ einen unter allen $U(x, \Lambda)$ invarianten Vektor $\Omega$ („das Vakuum“), der bis auf skalare Faktoren eindeutig bestimmt sei. Er erfüllt dann $P(E) \Omega=\Omega \forall E>0$.

Wir setzen voraus, daß die lokalen Algebren irreduzibel auf dem Hilbertraum wirken: Ist $\mathfrak{A}^{\text {lok }}$ die Vereinigung aller $\mathfrak{A}(\mathcal{O})$ für beschränkte Gebiete $\mathcal{O}$, so soll $\mathfrak{A}^{\text {lok }} \Omega$ dicht in $\mathcal{H}$ sein.

In unserem Zusammenhang werden wir oft nicht allgemeine Gebiete $\mathcal{O}$, sondern spezieller um den Koordinatenursprung zentrierte Standard-Doppelkegel mit Radius $r$ betrachten, die wir als

$$
\mathcal{O}_{r}=\left\{x \in \mathcal{M}|| x^{0}|+| \vec{x} \mid<r\right\}
$$

notieren. Die zugehörigen Algebren bezeichnen wir kurz mit $\mathfrak{A}(r):=\mathfrak{A}\left(\mathcal{O}_{r}\right)$.

Wir haben die lokalen Algebren $\mathfrak{A}(\mathcal{O})$ als von-Neumann-Algebren vorausgesetzt, um eine weitere mathematische Struktur zur Verfügung zu haben, nämlich ihren Prädualraum: Der Raum der normalen (d.h. linearen und ultraschwach stetigen) Funktionale auf $\mathfrak{B}(\mathcal{H})$ sei mit $\Sigma$ bezeichnet; er ist identisch mit dem Raum der Spurklasseoperatoren $\mathfrak{B}_{1}(\mathcal{H})$ auf $\mathcal{H}$, wobei $\rho \in \mathfrak{B}_{1}(\mathcal{H})$ ein $\sigma \in \Sigma$ induziert durch

$$
\sigma(\cdot)=\operatorname{tr}(\rho \cdot) \text {. }
$$

$\Sigma$ wird mit der Supremumsnorm (entsprechend der Spurnorm auf $\mathfrak{B}_{1}(\mathcal{H})$ ) zu einem Banachraum, dessen Dualraum gerade $\mathfrak{B}(\mathcal{H})$ ist:

$$
\Sigma^{*}=\mathfrak{B}(\mathcal{H}), \quad \text { auch notiert als } \Sigma=\mathfrak{B}(\mathcal{H})_{*} .
$$

Die positiven normierten Elemente von $\Sigma$ können als physikalische Zustände des betrachteten Systems gedeutet werden. In unserer Analyse ist noch der Raum der energiebeschränkten normalen Funktionale wichtig: Für $E>0$ sei

$$
\Sigma(E):=P(E) \Sigma P(E) ;
$$

dabei schreiben wir $P(E) \sigma P(E)=\sigma(P(E) \cdot P(E))$ für $\sigma \in \Sigma$. Man hat dann

$$
\Sigma(E)^{*}=P(E) \mathfrak{B}(\mathcal{H}) P(E) .
$$

Die $\Sigma(E)$ bilden eine Präkogarbe im Sinne von Anhang 2.A. 


\subsubsection{Wightman'sche Quantenfeldtheorie}

Die Wightman'sche Quantenfeldtheorie [SW64] formuliert man mit Hilfe von Feldern (unbeschränkten Operatoren) $\phi(f)$. Wir beschränken uns hier auf den Fall von Bose-Feldern; sie bzw. ihre selbstadjungierten Funktionen repräsentieren dann die physikalischen Observablen. Genauer betrachtet man folgende Struktur:

Zunächst ist ein Hilbertraum $\mathcal{H}$ gegeben, auf dem eine unitäre, stark stetige Darstellung $U(x, \Lambda)$ von $\mathfrak{P}$ wirkt. Wie in Abschnitt 1.3 .1 bezeichnen wir die Generatoren der Translation mit $P_{\mu}$; sie mögen der Spektrumsbedingung genügen. Außerdem gebe es einen bis auf einen Faktor eindeutigen $U$-invarianten Vektor $\Omega \in \mathcal{H}$.

Unter einem Quantenfeld bzw. einem Satz von Quantenfeldern $\phi_{1}, \ldots, \phi_{n}$ versteht man nun folgendes:

- Die $\phi_{j}$ sind operatorwertige temperierte Distributionen, d.h. sie sind lineare Abbildungen von $\mathcal{S}(\mathcal{M})$ in die Menge der (nicht notwendig beschränkten) linearen Operatoren auf $\mathcal{D}$, wobei $\mathcal{D} \subset \mathcal{H}$ ein dichter Unterraum ist. Man betrachtet also unbeschränkte Operatoren $\phi_{j}(f)$ auf einem gemeinsamen Definitionsbereich $\mathcal{D}$. Für $\xi, \xi^{\prime} \in \mathcal{D}$ ist das Matrixelement $\left(\xi \mid \phi_{j}(f) \xi^{\prime}\right)$ stetig in $f$ bezüglich der LaurentSchwartz-Topologie (,Temperiertheit“).

- Der Definitionsbereich $\mathcal{D}$ ist nicht nur dicht in $\mathcal{H}$, sondern auch stabil unter Anwendung der $U(x, \Lambda)$ und $\phi_{j}(f)$; ferner ist $\Omega \in \mathcal{D}$.

- Die adjungierten Operatoren $\phi_{j}(f)^{*}$ sind ebenfalls in der Menge der Felder enthalten:

$$
\phi_{j}(f)^{*}=\phi_{k}(\bar{f}) \quad \text { mit zu } j \text { geeignet gewähltem } k .
$$

Dies gilt als Operatorgleichung auf $\mathcal{D}$.

- Die $\phi_{j}$ erfüllen kausale Vertauschungsrelationen, d.h. für Testfunktionen $f, g$ mit raumartig getrenntem Träger gilt

$$
\left[\phi_{j}(f), \phi_{k}(g)\right]=0, \quad j, k=1 \ldots n,
$$

wieder als Gleichung auf $\mathcal{D}$.

- Es gibt eine endlichdimensionale Matrixdarstellung $S_{j k}$ von $\mathfrak{L}$, so daß

$$
U(x, \Lambda) \phi_{j}(f) U(x, \Lambda)^{*}=\sum_{k=1}^{n} S_{j k}\left(\Lambda^{-1}\right) \phi_{k}\left(f_{x, \Lambda}\right),
$$

wobei

$$
f_{x, \Lambda}(y)=f\left(\Lambda^{-1}(y-x)\right) .
$$

Zusätzlich wird oft noch gefordert, daß die $\phi_{j}(f)$ eine Vollständigkeits- oder Irreduzibilitätseigenschaft besitzen, analog zu dem, was wir im algebraischen Rahmen für die Algebra $\mathfrak{A}^{\text {lok }}$ gefordert hatten: Die Menge der Polynome der $\phi_{j}(f)$ (mit beliebigen Testfunktionen $f$ ) soll, angewandt auf $\Omega$, eine in $\mathcal{H}$ dichte Menge ergeben. Dies läßt sich ggf. immer erreichen, indem man $\mathcal{H}$ durch einen abgeschlossenen Teilraum ersetzt. 
Häufig betrachtet man statt der $\phi(f)$ auch punktartig lokalisierte Felder $\phi(x)$; diese sind dann nicht als Operatoren, sondern nur als quadratische Formen auf $\mathcal{D} \times \mathcal{D}$ definiert. Die $\phi(f)$ ergeben sich aus ihnen durch „Verschmieren“ mit einer Testfunktion:

$$
\phi(f)=\int d^{s+1} x f(x) \phi(x) .
$$

Wir werden diese heuristische Formel in Abschnitt 3.4 genauer präzisieren. Die obigen Bedingungen lassen sich fast alle in analoger Weise auch für die $\phi(x)$ aufstellen. Probleme bereitet dabei aber die Lokalitätsforderung: Da das Produkt zweier quadratischer Formen sich im allgemeinen nicht definieren läßt, machen Kommutatorrelationen wie (1.3.13) für die $\phi(x)$ keinen Sinn. Für eine exakte Formulierung muß man hier auf die Operatoren $\phi(f)$ ausweichen. 



\section{Teil I}

\section{Punktfelder}





\section{Kapitel 2}

\section{Phasenraumstruktur}

Wir beginnen unsere Analyse der Punktfeldeigenschaften von Quantenfeldtheorien mit einer Untersuchung des Kurzabstandsverhaltens der Observablenalgebren.

Während die Konstruktion von ausgedehnten Observablen aus Punktfeldern in der Literatur gut untersucht ist [DSW86, BY90], sind die Ergebnisse zum umgekehrten Prozeß, nämlich der Beschreibung von Punktfeldern durch Approximation mit immer besser lokalisierten Observablen [FH81, Wol85, RW86, Sum87], bisher unvollständig: Sie lassen nur wenig detaillierte Aussagen über die betreffenden Punktfelder zu. Wir nähern uns diesem Problem durch Analyse der Phasenraumstruktur der Theorie.

Phasenraumkriterien [HS65, BW86, BP90] sind im Rahmen der algebraischen Feldtheorie ein wohlbekanntes Mittel zur Beschreibung des Verhaltens von Theorien bei sehr großen oder sehr kleinen Abständen. Sie verlangen grob gesagt folgendes: Bei gleichzeitiger Einschränkung der auszuführenden Messungen im Impuls- und Ortsraum lassen sich die Meßergebnisse sehr gut durch Auswertung auf einem endlichdimensionalen Raum von Observablen bzw. Zuständen approximieren.

Wir untersuchen solche Phasenraumeigenschaften hier speziell im Hinblick auf das Kurzabstandsverhalten der Theorie - zunächst heuristisch und an einem nichtrelativistischen Beispiel, dann mathematisch präzisiert. Wir geben dann ein Kriterium an, das speziell auf asymptotisch kleinen Längenskalen sensitiv ist und das solche Quantenfeldtheorien selektiert, die sich in diesem Bereich in gewisser Weise „regulär“ verhalten und deren Observablenalgebren hier quasi durch endlich viele Observablen erzeugt werden.

Dieses Kriterium wird uns in späteren Kapiteln ermöglichen, zu der algebraischen Theorie assoziierte Punktfelder zu konstruieren. 


\subsection{Das Kurzabstandsverhalten von Quantenfeldtheorien}

Zur Analyse des Kurzabstandsverhaltens einer allgemeinen Quantenfeldtheorie gehen wir, wie angekündigt, von einer Theorie in der algebraischen Formulierung aus. Wir betrachten physikalisch also Observablen, die in endlich großen Gebieten der Raumzeit (etwa Doppelkegeln) lokalisiert sind, und untersuchen die Struktur der Theorie für immer kleinere Durchmesser der Lokalisationsgebiete.

In einfachen Fällen, wie der freien Feldtheorie oder (näherungsweise) dilatationsinvarianten Modellen, wird man dabei vermuten, daß im Kurzabstandslimes die der Theorie zugrundeliegenden Punktfelder als „idealisierte Observablen“ sichtbar sind; tatsächlich erwartet man hier sogar eine Eins-zu-eins-Beziehung zwischen dem algebraischen Modell und dem unterliegenden Punktfeld-Formalismus.

Im allgemeinen allerdings ist der algebraische Rahmen deutlich reichhaltiger als der $\mathrm{Zu}-$ gang über Punktfelder: Er könnte auch Observablen beschreiben, die nicht aus punktartig lokalisierten Größen aufgebaut sind, sondern die grundsätzlich ausgedehnte Lokalisierungsgebiete benötigen; man kann hierbei an feldartige Größen, die längs Wegen (MandelstamStrings), Schleifen (Wilson-Loops) etc. lokalisiert sind, denken. Man wird also nicht erwarten, daß solche Theorien durch die Angabe zugehöriger Punktfelder eindeutig charakterisiert sind.

Wir interessieren uns hier allerdings nicht für solche ausgedehnt lokalisierten Größen, sondern sind lediglich an den in der Theorie enthaltenen (respektive dazu assoziierten) Punktfeldern, also am „Feldinhalt" interessiert. Dieser beschreibt die Theorie zwar möglicherweise nicht vollständig, liefert aber zumindest eine Möglichkeit zur Klassifikation.

Es ist also zunächst unsere Aufgabe, die Relationen zwischen dem algebraischen Bild und dem Punktfeld-Formalismus genauer zu klären. Dabei ist die eine Richtung, nämlich die Konstruktion von algebraischen Theorien aus gegebenen Systemen von Punktfeldern, in der Literatur gut untersucht (siehe [BY90] und die dort zitierten Referenzen). Die Punktfelder $\phi(x)$ werden zunächst mit Testfunktionen $f$ zu i.allg. unbeschränkten Operatoren (Wightman-Feldern) $\phi(f)$ ausintegriert:

$$
\phi(f)=\int f(x) \phi(x) d^{s+1} x, \quad f \in \mathcal{S}(\mathcal{M}) .
$$

Dann geht man zu beschränkten Funktionen dieser Operatoren über; im Fall selbstadjungierter Wightman-Felder werden die lokalen Algebren $\mathfrak{A}(\mathcal{O})$ von den unitären Operatoren

$$
e^{i \phi(f)}, \quad \operatorname{supp} f \subset \mathcal{O}
$$

mit reellwertigen Testfunktionen $f$ erzeugt.

Weit weniger untersucht ist die umgekehrte Richtung, also die Konstruktion gegebener Punktfelder aus einem vorgegebenen lokalen Netz $\mathfrak{A}(\mathcal{O})$. Hier hat man offenbar die Algebren für Gebiete $\mathcal{O}$ zu analysieren, die sich auf einen Punkt $x$ zusammenziehen. Heuristisch könnte man die gesuchten Punktfelder einfach im Durchschnitt der Algebren $\mathfrak{A}(\mathcal{O})$ über alle Umgebungen $\mathcal{O}$ von $x$ vermuten:

$$
\phi(x) \stackrel{?}{\in} \bigcap_{\mathcal{O} \ni x} \mathfrak{A}(\mathcal{O}) .
$$


Tatsächlich stellt sich aber unter recht allgemeinen Bedingungen heraus, daß der Durchschnitt auf der rechten Seite nur Vielfache des Einsoperators enthält [BV95, p.1215]. Dies ist insofern nicht weiter verwunderlich, als die gesuchten Punktfelder singuläre Objekte sein sollten, nicht beschränkte Operatoren wie die Elemente der lokalen Algebren.

Diese Singularität der Felder kann heuristisch als Folge der Unschärferelation verstanden werden: Eine in Raum und Zeit beliebig gut lokalisierte Messung muß im Impulsraum völlig delokalisiert sein, also einen „unendlich großen“ Energie-Impuls-Übertrag besitzen. Das singuläre Verhalten der Felder ist also in ihrem Hochenergieverhalten begründet; dämpft man dieses durch einen geeigneten Operator, etwa einen Spektralprojektor $P(E)$ des Hamiltonoperators, dann sollten die resultierenden Felder $P(E) \phi(x) P(E)$ beschränkt sein. In allen bisher konstruierten Modellen weiß man sogar, daß die Felder Schranken der Art

$$
\|P(E) \phi(x) P(E)\|<E^{l} \cdot \text { const. }
$$

mit gewissem $l>0$ erfüllen (siehe z.B. [DF77]).

Aus diesem Grund scheint es natürlich, auch in (2.1.3) eine Energiedämpfung einzuführen, um das Scheitern des naiven Ansatzes zu umgehen. So betrachteten Fredenhagen und Hertel [FH8]] für $l>0$ und $R=(1+H)^{-1}$ die Räume

$$
\bigcap_{\mathcal{O} \ni x} \overline{R^{l} \mathfrak{A}(\mathcal{O}) R^{l}}
$$

und zeigten, daß man die Elemente dieser Durchschnitte als energiegedämpfte lokale Punktfelder $R^{l} \phi(x) R^{l}$ auffassen kann. In gewisser Weise erhält man sogar alle mit der Theorie assoziierten Punktfelder mit Hilfe der Struktur (2.1.5). Diese Analysemethoden wurde später von verschiedenen Autoren [RW86, Wol85, Sum87] noch auf größere Klassen von Feldern ausgedehnt. Alle diese Arbeiten zeigen, daß man die Felder als Grenzwerte lokaler Observablen unter Energiedämpfung suchen muß. Sie ergeben jedoch kaum Aussagen über weitere Strukturen der erhaltenen Wightman-Felder.

Die Idee der Energiedämpfung ist noch in einem anderen Zusammenhang von Bedeutung, der zunächst unabhängig von der Frage der Punktfelder untersucht wurde, nämlich den sogenannten Kompaktheits- oder Nuklearitätseigenschaften. Zuerst diskutierten Haag und Swieca [HS65] die Struktur lokaler Algebren bei endlichen Radien und beschränkten Energien, ausgehend von folgender heuristischer Idee:

Im Rahmen eines halbklassischen Bilds der (nichtrelativistischen) Quantenmechanik erwartet man, daß zu jedem endlichen Gebiet des klassischen Phasenraums nur eine endliche Zahl von Zuständen des Quantensystems gehören: Jeder Quantenzustand eines Einteilchensystems mit $s$ Freiheitsgraden belegt eine „Phasenraumzelle“ der Größe $h^{s}$. I In der Quantenfeldtheorie würde man analog die Zustände betrachten, die durch lokale Operationen aus dem Vakuum erzeugt werden können, und diese dann in der Energie beschränken: Die Räume

$$
P(E) \mathfrak{A}(r) \Omega
$$

\footnotetext{
${ }^{1}$ Eine quantitativere Fassung dieser Aussage ist z.B. die Bohr-Sommerfeld'sche Quantisierungsbedingung $\oint p_{i} d q_{i}=n h$.
} 
von Zuständen gehören in diesem Sinn zu einem endlichen Phasenraumvolumen $E \cdot r$ und sollte daher endlichdimensional sein, wenn sich die Ergebnisse der nichtrelativistischen Theorie direkt übertragen lassen. Tatsächlich ist dies aber nicht der Fall, wie der Satz von Reeh und Schlieder zeigt: Es gilt $\overline{P(E) \mathfrak{A}(r) \Omega}=P(E) \mathcal{H}$, d.h. mit den Zuständen (2.1.6) lassen sich beliebige energiebeschränkte Zustände approximieren (sicher nicht nur endlich viele).

Dennoch sollten nach Haag und Swieca die endlichdimensionalen Strukturen aus der Quantenmechanik zumindest in einem approximativen Sinn sichtbar sein. Die Autoren postulierten, daß die Mengen

$$
P(E) \mathfrak{A}(r)_{1} \Omega
$$

„fast endlichdimensional“, nämlich in der Hilbertraum-Norm präkompakt sein sollten, um eine sinnvolle Teilcheninterpretation der Quantenfeldtheorie zuzulassen.

Diese Ideen wurden später von vielen anderen Autoren ausgebaut. Wegen des problematischen Begriffs der Lokalisierung von Zuständen empfiehlt es sich dabei, zur Lokalisierung von Observablen überzugehen bzw. allgemein energiegedämpfte Zustände zu betrachten, die aber nur auf lokalisierten Operatoren ausgewertet werden. Hier lassen sich analoge Resultate erzielen. So zeigten Buchholz und Porrmann [BP90], daß in gewissen Modellen der freien Feldtheorie die Abbildungen

$$
A \mapsto e^{-\beta H} A e^{-\beta H} \quad(A \in \mathfrak{A}(r))
$$

nuklear sind (d.h. als Summe von Rang-1-Abbildungen geschrieben werden können), und konnten Abschätzungen für ihren Nuklearitätsindex angeben.

Kompaktheits- und Nuklearitätskriterien wurden in der Literatur einerseits zur Kurzabstandsanalyse von Quantenfeldtheorien eingesetzt, etwa zur Beschreibung eines Skalenlimes [BV.95, Buc.96], andererseits auch zur Analyse von Feldtheorien bei großen Abständen $r$, insbesondere zur Untersuchung thermodynamischer Aspekte. So folgt aus Nuklearitätsbedingungen die Existenz von KMS-Temperaturzuständen [B.J89] sowie die sogenannte Split-Eigenschaft [BW86], welche die Interpretation endlich ausgedehnter Teilsysteme in der Theorie erlaubt. Diese Eigenschaften werden nicht durch die allgemeinen Axiome der Quantenfeldtheorie (z.B. die Wightman-Axiome) garantiert, sondern man findet Gegenbeispiele z.B. in freien Feldtheorien mit unendlich vielen Teilchensorten, deren Massen nur sehr langsam anwachsen [B.I86]. Insofern scheinen Nuklearitätsbedingungen geeignet, um aus der Menge aller mathematisch möglichen Quantenfeldtheorien diejenigen auszuzeichnen, die eine sinnvolle physikalische Interpretation zulassen.

Der Zusammenhang zwischen den Phasenraumeigenschaften und der Existenz von Punktfeldern wurde zuerst von Haag erwähnt [Haa.93] und später von Haag und Ojima genauer formuliert [H()96]: Die Autoren vermuteten (und begründeten im Beispiel des masselosen freien Feldes heuristisch), daß die analog zum Obigen gebildeten Räume

$$
P(E) \mathfrak{A}(r) P(E)
$$

nicht nur "fast endlichdimensional" sein sollten, sondern daß die gesuchten Punktfelder quasi eine Basis dieser beinahe endlichdimensionalen Räume bilden. Genauer betrachteten sie die hierzu dualen Räume

$$
P(E) \Sigma(r) P(E)
$$


(wobei $\Sigma(r)=\Sigma\lceil\mathfrak{A}(r)$ ), von denen sie postulierten, daß sie durch eine endlichdimensionale Basis von $E$ - und $r$ - unabhängigen Zuständen $\sigma_{j}$ bis zu einer vorgegebenen Genauigkeit im Limes $r \rightarrow 0$ gut approximiert werden können. Die Zahl der Elemente dieser „Basis“ erhöht sich, wenn man eine bessere Approximation fordert; so erhält man eine Hierarchie von Zuständen $\sigma_{j}$ als Basis der Räume (2.1.10), und die gesuchten Punktfelder ergeben sich quasi als Basis des Dualraums. Diese Ideen wurden in [Bos.98] aufgegriffen und präzisiert; das zu fordernde Kriterium konnte in Modellen der freien Feldtheorie in mindestens 3 räumlichen Dimensionen mathematisch streng etabliert werden. Die vorliegende Arbeit baut auf diesen Konzepten auf.

Nicht unerwähnt sollen einige konkretere Ansätze bleiben, die in gewissen Modellen eine direktere Konstruktion der Punktfelder erlauben: Im Fall von dilatationsinvarianten Theorien hat man als zusätzliches Analysemittel die Darsteller $D(\lambda)$ der Dilatationen zur Verfügung. So bemerkten Buchholz und Fredenhagen [BF77], daß man (heuristisch) folgendes Verhalten der $A \in \mathfrak{A}(\mathcal{O})$ finden sollte:

$$
D(\lambda) A D(\lambda)^{-1}=(\Omega \mid A \Omega) \mathbf{1}+\lambda \phi(0)+o(\lambda) \quad \text { für } \lambda \rightarrow 0
$$

mit einem Punktfeld $\phi(0)$. Fredenhagen und Jörß [F.I.96] führten eine ähnliche Konstruktion in einem speziellen Modell, der zweidimensionalen konformen chiralen Theorie eines reellen skalaren Teilchens, sehr explizit durch; sie erhalten Punktfelder $\phi(0)$ als Limiten

$$
\lambda^{-n} D(\lambda) A \Omega \underset{\lambda \rightarrow 0}{\longrightarrow} \phi(0) \Omega .
$$

Diese Vorgehensweise läßt noch Verallgemeinerungen zu [Jör96], bleibt aber prinzipiell auf dilatationsinvariante Modelle beschränkt: Unitäre Darsteller $D(\lambda)$ der Dilatationen sind im allgemeinen nicht verfügbar, nicht einmal in Theorien freier massiver Teilchen.

\subsection{Asymptotische Phasenraumeigenschaften}

Der im letzten Abschnitt zusammengefaßten Wissensstand über die Struktur von Quantenfeldtheorien bei kleinen Abständen läßt sich im wesentlichen auf folgende drei Aspekte reduzieren:

- Neben der Lokalisierung der Observablen benötigt man als weitere Struktur eine Energiebeschränkung, um den Zusammenhang zwischen Feldern und lokalen Algebren herzustellen.

- Die gleichzeitig in Ort und Energie beschränkten Objekte der Theorie - Räume von Observablen oder Zuständen - besitzen eine sehr einfache, beinahe endlichdimensionale Gestalt.

- Punktfelder erhält man aus diesen Strukturen, indem man zum Limes kleiner Abstände $r$ übergeht.

Unser Ziel ist es, unter Verwendung dieser Aspekte ein Kriterium zu entwickeln, das es erlaubt, Punktfelder aus einem Netz von Algebren zu konstruieren. Dies geschieht zunächst heuristisch. 
Wir gehen dazu aus von einem lokalen Netz $\mathfrak{A}(\mathcal{O})$ bzw. den zu Doppelkegeln $\mathcal{O}_{r}$ gehörenden Algebren $\mathfrak{A}(r) \equiv \mathfrak{A}\left(\mathcal{O}_{r}\right)$. (Es reicht für die Analyse der Punktfeldeigenschaften aus, um den Koordinatenursprung zentrierte Doppelkegel zu betrachten, da die betrachtete Theorie als translationsinvariant angenommen wird.) Die Operatoren in $\mathfrak{A}(r)$ entsprechen also Messungen, die innerhalb des Raum-Zeit-Gebietes $\mathcal{O}_{r}$ stattfinden. Mit $\Sigma$ bezeichnen wir

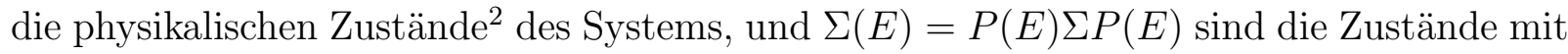
eingeschränkter Energie $E$. Wir interessieren uns für das Verhalten der Erwartungswerte

$$
\sigma(A), \quad \text { wobei } \sigma \in \Sigma(E), A \in \mathfrak{A}(r)
$$

bei kleinen Abständen $r$ und nicht allzu großen Energien $E$; diese Erwartungswerte sollen analysiert werden, und zwar in einer Weise, die nicht vom speziell gewählten Zustand $\sigma$ bzw. der Observablen $A$ abhängt.

Die zu erwartende Situation skizzieren wir zunächst an einem Beispiel aus der Quantenmechanik: Wir betrachten ein nichtrelativistisches, freies Teilchen in einer Dimension. Die energiebeschränkten Zustände entsprechen hier Hilbertraum-Vektoren der Form $\varphi=P(E) \varphi_{0}$, und als lokalisierte Observablen verwenden wir Funktionen $f(Q)$ des Ortsoperators mit kompaktem Träger. Die Funktionen $\varphi$ haben beschränkten Träger im Impulsraum und sind daher im Ortsraum holomorph, d.h. wir können sie als Potenzreihe schreiben:

$$
\varphi(x)=\sum_{n} a_{n} x^{n}=\sum_{n} \frac{\varphi^{(n)}(0)}{n !} x^{n} .
$$

Die Koeffizienten der Reihe lassen sich dabei als „Skalarprodukte“ mit Deltafunktionen darstellen:

$$
\varphi^{(n)}(0)=(-1)^{n} \int \delta^{(n)}(x) \varphi(x) d x=(-1)^{n}\left\langle\delta^{(n)} \mid \varphi\right\rangle .
$$

Ist nun $\psi$ eine weitere Wellenfunktion, die im Ortsraum beschränkten Träger besitzt, so erhält man für ihr Skalarprodukt mit $\varphi$ die Entwicklung

$$
\langle\psi \mid \varphi\rangle=\sum_{n} a_{n}\left\langle\psi \mid x^{n}\right\rangle=\sum_{n} \frac{(-1)^{n}}{n !}\left\langle\psi \mid x^{n}\right\rangle\left\langle\delta^{(n)} \mid \varphi\right\rangle .
$$

Aufgrund des beschränkten Trägers von $\psi$ sind die Skalarprodukte $\left\langle\psi \mid x^{n}\right\rangle$ hier wohldefiniert.

Nun sei $A=f(Q)$ eine lokalisierte Observable. Im interessierenden Erwartungswert $\sigma(A)=\langle\varphi|f(Q)| \varphi\rangle$ können wir die Formel (2.2.4) zweimal anwenden und erhalten

$$
\begin{aligned}
\sigma(A)=\langle\varphi \mid f(Q) \varphi\rangle & =\sum_{m, n} \frac{(-1)^{m+n}}{m ! n !}\left\langle\varphi \mid \delta^{(m)}\right\rangle\left\langle x^{m} \mid f(Q) x^{n}\right\rangle\left\langle\delta^{(n)} \mid \varphi\right\rangle \\
= & \sum_{m, n}\left\langle\varphi \mid \frac{(-1)^{m+n}}{m ! n !} \delta^{(m)}\right\rangle\left\langle\delta^{(n)} \mid \varphi\right\rangle\left\langle x^{m}|f(Q)| x^{n}\right\rangle=\sum_{m, n} \sigma\left(A_{m n}\right) \sigma_{m n}(A) \\
& \text { mit } A_{m n}:=\frac{(-1)^{m+n}}{m ! n !}\left|\delta^{(m)}\right\rangle\left\langle\delta^{(n)}\right| \quad \text { und } \quad \sigma_{m n}:=\left\langle x^{m}|\cdot| x^{n}\right\rangle .
\end{aligned}
$$

${ }^{2}$ Technisch ist $\Sigma$ die Menge der schwach-*-stetigen Funktionale, also der Spurmatrizen, über $\mathfrak{B}(\mathcal{H})$.

${ }^{3}$ Indem wir im Ausdruck $\left\langle\delta^{(n)} \mid \varphi\right\rangle$ einen Energieprojektor $P(E)$ auf die linke Seite bringen, können wir die Deltafunktion hier durch einen regulären Zustandsvektor im Hilbertraum ersetzen. 
Wir haben den Erwartungswert $\sigma(A)$ also in eine Reihe aufgespalten, deren einzelne Terme durch Auswertung von $\sigma$ auf „Standardobservablen“ $A_{m n}$ und von $A$ auf zugehörigen „Standardfunktionalen“ $\sigma_{m n}$ entstehen. Diese $A_{m n}$ und $\sigma_{m n}$ hängen nicht von der speziellen Wahl von $\sigma$ und $A \mathrm{ab}$, sondern können quasi als intrinsische Größen der Theorie angesehen werden.

Weiterhin sieht man, daß die einzelnen Terme der Reihe im betrachteten asymptotischen Bereich ein charakteristisches Verhalten zeigen: Betrachtet man nur Observablen $A$, die in einem Intervall der Länge $r$ lokalisiert sind, so verhält sich die korrespondierende Norm des Funktionals $\sigma_{m n}$ offenbar wie $r^{m+n+1}$. Ist umgekehrt $p$ der für den Zustand $\sigma$ maximal zulässige Impulswert, so divergiert der Anteil von $A_{m n}$ im Limes großer $p$ wie $p^{m+n+1}$. (Im nichtrelativistischen Fall ist dabei $p \sim \sqrt{E}$, während wir im unten zu betrachtenden relativistischen Fall $p \sim E$ erwarten.) Wir erhalten also eine Art Hierarchie von Approximationstermen $\sigma_{m n} A_{m n}$ für den Erwartungswert $\sigma(A)$, wobei jeweils nur endlich viele zu einem gegebenen Kurzabstandsverhalten $r^{\gamma}$ gehören.

Wir werden diese Überlegungen nun auf die Situation in der Quantenfeldtheorie übertragen und dort allgemein lokale Algebren $\mathfrak{A}(r)$ und Räume von energiebeschränkten Funktionalen $\Sigma(E)$ betrachten. Uns interessiert das Verhalten bei kleinen Abständen, also $r \rightarrow 0$; gleichzeitig darf die Energie $E$ der zulässigen Zustände anwachsen, jedoch nicht zu stark, so daß das „Phasenraumvolumen“, d.h. das Produkt $E \cdot r$, klein bleibt. Am einfachsten läßt sich dies so formulieren, daß wir den Grenzfall $E r \rightarrow 0$ betrachten. (Es erscheint sinnvoll, sich hier auf die skaleninvariante Größe $E \cdot r$ zu stützen, da in dem interessierenden Hochenergielimes die Massen der in der Theorie enthaltenen Teilchen keine Rolle spielen sollten.)

Unsere Vorstellung ist nun, ausgehend vom eben diskutierten nichtrelativistischen Modell, folgende: Je kleiner $E r$ wird, desto ungenauer wird man verschiedene Observablen $A$ bei einer gegebenen Meßauflösung unterscheiden können. In erster Näherung kann die Messung $A$ gut durch den zugehörigen Anteil des Einsoperators $\omega(A) \mathbf{1}$ beschrieben werden; hier ist $\omega$ ein gewisses Funktional, das in konkreten Modellen durch den Vakuumzustand gegeben ist. Es sollte also gelten, daß

$$
\sigma(A) \approx \sigma(\omega(A) \mathbf{1}) \text { für kleine } \operatorname{Er} .
$$

In der Tat findet man im allgemeinen, daß etwas präziser

$$
\sup _{\sigma \in \Sigma(E)_{1}} \sup _{A \in \mathfrak{A}(r)_{1}}|\sigma(A)-\sigma(\mathbf{1}) \omega(A)| \underset{E r \rightarrow 0}{\longrightarrow} 0 .
$$

In diesem Sinne tritt im Grenzfall nur eine unabhängige Observable (der Einsoperator) auf. Will man mehr Observablen unterscheiden, so muß man die Meßaufösung mit Verkleinerung der Skala erhöhen. Wir berücksichtigen dies durch einen Faktor $(E r)^{-1}$ in (2.2.7); man stellt fest, daß

$$
\sup _{\sigma, A} \frac{1}{E r}|\sigma(A)-\sigma(\mathbf{1}) \omega(A)| \not \rightarrow 0
$$

Auf diese Weise entdeckt man also weitere Anteile der Erwartungswerte. Um diese zu kompensieren, subtrahiert man (in Beispielen) eine weitere Standardobservable $\phi$ mit einem 
passenden Koeffizienten $\tau(A)$ und erhält

$$
\sup _{\sigma, A} \frac{1}{E r}|\sigma(A)-\sigma(\mathbf{1}) \omega(A)-\sigma(\phi) \tau(A)| \underset{E r \rightarrow 0}{\longrightarrow} 0 .
$$

Dieses Verfahren läßt sich nun für immer höhere Meßauflösungen fortsetzen; man ersetzt den Faktor $(E r)^{-1}$ durch ein allgemeineres $(E r)^{-\gamma}$ und erhält dazu $n$ Approximationsterme, so daß

$$
\sup _{\sigma, A} \frac{1}{(E r)^{\gamma}}\left|\sigma(A)-\sum_{j=1}^{n} \sigma\left(\phi_{j}\right) \sigma_{j}(A)\right| \underset{E r \rightarrow 0}{\longrightarrow} 0 .
$$

Dabei wird $n$ sich typischerweise mit wachsendem $\gamma$ vergrößern.

Wir finden in unserem Grenzwert kleinen Phasenraumvolumens also, daß sich die Erwartungswerte $\sigma(A)$ durch eine Summe bzw. Reihe von Erwartungswerten von StandardObservablen $\phi_{j}$ darstellen lassen, also etwa

$$
\sigma(A) \approx \sum_{j} \sigma\left(\phi_{j}\right) \sigma_{j}(A) .
$$

Je höher man im Limes $E r \rightarrow 0$ die Meßauflösung wählt, desto mehr Terme der (im allgemeinen unendlichen) Reihe benötigt man, um die Meßergebnisse zu approximieren.

(Im oben besprochenen nichtrelativistischen Beispiel haben wir die Reihe (2.2.11) konkret durch eine Potenzreihenentwicklung erhalten; auch in relativistischen Modellen freier Teilchen ist dies sehr ähnlich. Es sei aber betont, daß der hier gewählte Zugang allgemeiner ist - er läßt auch Approximationsterme zu, deren Er-Verhalten nicht durch ganzzahlige Potenzen $(E r)^{\gamma}$ beschrieben wird, sondern z.B. durch irrationale Exponenten oder logarithmische Faktoren.)

Da die Güte der Approximation nicht vom speziellen $\sigma$ oder $A$ abhängt, könnte man auch von einer Entwicklung nicht der einzelnen Erwartungswerte, sondern „der Bildung von Erwartungswerten an sich" sprechen. Die Messungen in den lokalen Algebren $\mathfrak{A}(r)$ reduzieren sich auf die Messung endlich vieler Standard-Observablen $\phi_{j}$, die quasi als $r$ unabhängige „Erzeuger“ der Algebren angesehen werden können. Insofern liegt es nahe, daß es sich dabei um Objekte handelt, die am Punkt $x=0$ lokalisiert sind; tatsächlich handelt es sich im Beispiel der freien Feldtheorie außer dem Einsoperator um die der Theorie zugrundeliegenden Punktfelder sowie ihre Ableitungen und Normalprodukte (Wick-Produkte).

Wir werden das hier angedeutete Kriterium im nächsten Abschnitt zu präzisieren und in eine mathematisch auswertbare Form zu bringen haben. Dies wird uns später erlauben, die auftretenden Standard-Observablen $\phi_{j}$ als lokale Punktfelder zu identifizieren, die den Wightman-Axiomen genügen. Zunächst sei aber bezüglich des heuristischen Inhalts auf folgende Punkte hingewiesen, welche die hier untersuchte Struktur von derjenigen unterscheiden, die im Zusammenhang mit Phasenraumkriterien in der Literatur häufig betrachtet wird:

- Das halbklassische Argument einer Aufteilung des Phasenraums in „Zellen“ der Größe $h^{s}$, das ursprünglich die Motivation für die Einführung von Kompaktheitskriterien lieferte [HS65], spielt im hier betrachteten Zugang keine Rolle. Da unsere Analyse 
sich im Bereich kleiner „Phasenraumvolumina“ $E \cdot r$ abspielt, befindet man sich quasi in dem Bereich, in dem die Theorie nur noch von einer einzigen halbklassischen Phasenraumzelle bestimmt wird. Man sollte also nur noch einen einzigen Zustand des Systems (das Vakuum der Quantenfeldtheorie) vorfinden. Dies ist insofern tatsächlich realisiert, als die Reihe (2.2.11) in Beispielen nur einen führenden (im Limes $E r \rightarrow 0$ nicht verschwindenden) Term besitzt; interessant ist aber vor allem die Analyse der restlichen, unterschiedlich schnell verschwindenden Approximationsterme.

- Wir interessieren uns nicht für die Struktur der Theorie bei einem festen Radius $r$ und fester Energie $E$, sondern ausschließlich für das asymptotische Verhalten im Limes verschwindenden Phasenraumvolumens $(E \cdot r \rightarrow 0)$. Dies unterscheidet den hier gewählten Zugang von den Phasenraumbedingungen, die beispielsweise in [BW86] und [BP90] vorgeschlagen wurden. Von der postulierten Reihe (2.2.11) ist etwa im Beispiel der freien Feldtheorie nicht bekannt, ob sie bei festem $E$ und $r$ konvergiert - dies erweist sich für die Konstruktion der Punktfelder auch als irrelevant.

\subsection{Mathematische Formalisierung}

Wir werden nun die heuristischen Überlegungen des letzten Abschnitts zusammenfassen und in ihren mathematischen Kontext stellen. Dabei wird jedoch nicht auf alle technischen Details der verwendeten Strukturen eingegangen; eine ausführlichere Darstellung der Hintergründe findet sich in den Anhängen 2.A bis 2.0.

Unser Ausgangspunkt ist eine Quantenfeldtheorie in algebraischer Formulierung $(\mathcal{O} \mapsto$ $\mathfrak{A}(\mathcal{O})$, die den üblichen, in Abschnitt 1.3.1 genannten Axiomen genügt. Wie bisher betrachten wir insbesondere die Algebren $\mathfrak{A}(r)=\mathfrak{A}\left(\mathcal{O}_{r}\right)$. Mit den zwischen ihnen bestehenden Inklusionen $\left(\mathfrak{A}(r) \hookrightarrow \mathfrak{A}\left(r^{\prime}\right)\right.$ für $\left.r<r^{\prime}\right)$ bilden sie eine Präkogarbe

$$
\overline{\mathfrak{A}}=\{\mathfrak{A}(r)\} \quad \text { auf dem Intervall }(0,1] .
$$

(Das Intervall ist so gewählt, da wir das Verhalten der Theorie bei kleinen $r$ analysieren wollen.)

Ähnlich verfahren wir mit den energiebeschränkten normalen Funktionalen $\Sigma(E):=P(E) \Sigma P(E)$ : Sie bilden eine Präkogarbe

$$
\bar{\Sigma}=\{\Sigma(E)\} \quad \text { auf dem Intervall }[1, \infty)
$$

mit den Inklusionen $\Sigma(E) \hookrightarrow \Sigma\left(E^{\prime}\right)$ für $E<E^{\prime}$. (Wir betrachten das Hochenergieverhalten der Theorie, deshalb haben wir uns auf das Intervall $[1, \infty)$ eingeschränkt.) Dementsprechend bildet das mengentheoretische Produkt

$$
\bar{\Sigma} \times \overline{\mathfrak{A}}=\{\Sigma(E) \times \mathfrak{A}(r)\}
$$

eine Präkogarbe über $[1, \infty) \times(0,1]$. Die uns interessierende Operation „Auswertung lokaler Messungen" kann als bilineare Abbildung auf $\bar{\Sigma} \times \overline{\mathfrak{A}}$ verstanden werden:

$$
\begin{array}{ll}
\Xi: \quad \bar{\Sigma} \times \overline{\mathfrak{A}} \rightarrow \mathbb{C}, \\
(\sigma, A) \mapsto \sigma(A) .
\end{array}
$$


Sie ist verträglich mit den Inklusionsabbildungen und kann insofern als Abbildung auf der Präkogarbe bezeichnet werden.

Zur Formulierung des Kriteriums geht es darum, Approximation dieser Abbildung $\Xi \mathrm{zu}$ betrachten. Nach Abschnitt 2.2 sollen die approximierenden Terme „Rang-1-Abbildungen“ sein, die in unserem Kontext ebenfalls als bilineare Abbildungen auf der Präkogarbe $\bar{\Sigma} \times \overline{\mathfrak{A}}$ dargestellt werden können:

$$
\begin{aligned}
\psi_{A_{0} \sigma_{0}}: & \bar{\Sigma} \times \overline{\mathfrak{A}} \rightarrow \mathbb{C}, \\
& (\sigma, A) \mapsto \sigma\left(A_{0}\right) \sigma_{0}(A),
\end{aligned}
$$

wobei $\sigma_{0} \in \Sigma$ ein festes Funktional ist und $A_{0}$ eine Linearform über jedem der $\Sigma(E)$, die mit deren Inklusionen verträglich ist. $\left(A_{0}\right.$ ist also eine evtl. unbeschränkte quadratische Form auf $\bigcup_{E} \Sigma_{E}$ mit der Eigenschaft, daß $P(E) A_{0} P(E)$ für jedes $E>0$ beschränkt ist. Die später zu identifizierenden Punktfelder werden von diesem Typ sein.) Die obige Abbildung $\psi_{A_{0} \sigma_{0}}$ bezeichnen wir im folgenden oft abgekürzt mit $A_{0} \sigma_{0}$.

Wir werden unseren Formalismus also in der Sprache bilinearer Abbildungen auf $\bar{\Sigma} \times \overline{\mathfrak{A}}$ aufbauen. Allerdings dürfen nicht alle solchen Abbildungen in der Analyse zugelassen werden, sondern wir müssen uns auf in gewisser Weise „reguläre“ Abbildungen beschränken, um ausreichende Stetigkeitseigenschaften für die zu konstruierenden Punktfelder zu erhalten. Dazu soll eine geeignete Topologie auf $\bar{\Sigma} \times \overline{\mathfrak{A}}$ eingeführt werden; die „regulären“ bilinearen Abbildungen sind dann gerade stetig in dieser Topologie. Wir müssen an diesen Stetigkeitsbegriff folgende Anforderungen stellen:

- Die oben erwähnten Abbildungen $\Xi$ und $A_{0} \sigma_{0}$ sollen stetig sein, wenn $\sigma_{0}$ schwach-*stetig ist und $A_{0}$ auf jedem $\Sigma(E)$ beschränkt.

- Umgekehrt soll aus der Stetigkeit einer Abbildung $A_{0} \sigma_{0}$ folgen, daß $\sigma_{0}$ schwach-*stetig ist, und daß $\left\|P(E) A_{0} P(E)\right\|<\infty$.

Wie in Anhang 2.A argumentiert wird, gibt es Topologien auf $\bar{\Sigma} \times \overline{\mathfrak{A}}$, die diese Forderungen erfüllen; die für unsere Zwecke natürlichste ist die Finaltopologie bezüglich der Abbildungen

$$
\begin{aligned}
& m_{\mathrm{L}, \sigma}: \quad \mathfrak{A}(r) \rightarrow \Sigma(E) \times \mathfrak{A}(r), \quad A \mapsto(\sigma, A) \quad(\sigma \in \Sigma(E) \text { fest }), \\
& m_{\mathrm{R}, A}: \quad \Sigma(E) \rightarrow \Sigma(E) \times \mathfrak{A}(r), \quad \sigma \mapsto(\sigma, A) \quad(A \in \mathfrak{A}(r) \text { fest }) ;
\end{aligned}
$$

dies ist in Anhang [2.A näher erläutert und hier zunächst nicht weiter von Bedeutung. Wir bezeichnen die Menge aller bezüglich dieser Topologie stetigen, bilinearen Abbildungen auf $\bar{\Sigma} \times \overline{\mathfrak{A}} \operatorname{mit} \bar{\Psi}$.

Unsere Vorstellung ist es also, daß sich die Auswertung lokaler Messungen, also die Abbildung $\Xi \in \bar{\Psi}$, durch Summen von Termen der Form $A_{0} \sigma_{0} \in \bar{\Psi}$ approximieren läßt, allgemeiner gesprochen durch Abbildungen in $\bar{\Psi}$ von endlichem Rang.

Auch hier ist zunächst genauer zu klären, was wir unter „endlichem Rang“ verstehen wollen. Für eine solche Abbildung bei festem $E$ und $r$ ist das relativ klar: Zu jeder bilinearen Abbildung $\psi_{E, r}: \Sigma(E) \times \mathfrak{A}(r) \rightarrow \mathbb{C}$ können wir die Linksadjungierte $\psi_{\mathrm{L} E, r}$ definieren als

$$
\begin{aligned}
\psi_{\mathrm{L} E, r}: & \Sigma(E) \rightarrow \mathfrak{A}(r)_{*}, \\
& \sigma \mapsto \psi_{E, r}(\sigma, \cdot)
\end{aligned}
$$


und dann den Rang von $\psi_{E, r}$ als Dimension des Bildes dieser Abbildung (welches wir mit $\operatorname{Bild}_{\mathrm{L}} \psi_{E, r}$ bezeichnen) festlegen, sofern es endlichdimensional ist. Analog läßt sich natürlich auch eine Rechtsadjungierte definieren,

$$
\begin{aligned}
\psi_{\mathrm{R} E, r}: & \mathfrak{A}(r) \rightarrow \Sigma(E)^{*}, \\
& A \mapsto \psi_{E, r}(\cdot, A),
\end{aligned}
$$

und die Dimension ihres Bildes Bild ${ }_{\mathrm{R}} \psi_{E, r}$ zur Definition von Rang $\psi_{E, r}$ heranziehen. Es stellt sich (wie zu erwarten) heraus, daß stets $\operatorname{dim} \operatorname{Bild}_{\mathrm{L}} \psi_{E, r}=\operatorname{dim} \operatorname{Bild}_{\mathrm{R}} \psi_{E, r}$ gilt, siehe Anhang 2.B.

Uns interessieren aber nicht die Abbildungen bei festem $E$ und $r$, sondern ihr Verhalten im Grenzfall $E r \rightarrow 0$. Es ist hier nicht ausreichend, nur zu fordern, daß der Rang von $\psi_{E, r}$ für $E r \rightarrow 0$ beschränkt bleibt oder konstant ist, da es uns hauptsächlich auf die Interpretation des Bildes als Punktfelder $\left(\operatorname{Bild}_{\mathrm{R}} \psi\right)$ bzw. als deren duale Objekte $\left(\operatorname{Bild}_{\mathrm{L}} \psi\right)$ ankommt. Wir müssen daher von den approximierenden Abbildungen verlangen, daß Bild ${ }_{\mathrm{L}} \psi_{E, r}$ und $\operatorname{Bild}_{\mathrm{R}} \psi_{E, r}$ nicht nur endlichdimensional, sondern für kleine $E \cdot r$ sogar „als Vektorraum konstant" sind. Wir bemerken hier nur, daß sich solche Abbildungen für kleine $E \cdot r$ gerade als endliche Summen von Rang-1-Termen der Form (2.3.5) schreiben lassen:

$$
\psi=\sum_{j=1}^{J} \phi_{j} \sigma_{j} \quad \text { mit } \sigma_{j} \in \Sigma, \phi_{j} \in \bar{\Sigma}^{*} .
$$

Für eine „,basisfreie“ Definition dieser Eigenschaft sei auf Anhang 2.C verwiesen. Solche Abbildungen $\psi \in \bar{\Psi}$ nennen wir Abbildungen von asymptotisch endlichem Rang. Die Menge bzw. den Vektorraum aller dieser Abbildungen bezeichnen wir mit $\bar{\Psi}_{0}$.

Polynomiale Energieschranken Wir werden häufig noch eine weitere Bedingungen an die betrachteten Abbildungen $\psi \in \bar{\Psi}_{0}$ stellen, und zwar eine Einschränkung an ihr Hochenergieverhalten: Wir sagen, $\psi \in \bar{\Psi}_{0}$ genüge polynomialen Energieschranken (oder: ist polynomial energiebeschränkt), wenn für jedes $\phi \in \operatorname{Bild}_{\mathrm{R}} \psi$ ein $l>0$ existiert, so daß

$$
\|\phi\|_{E}=\|P(E) \phi P(E)\| \leq E^{l} \cdot \text { const. }
$$

Es ist dann (durch Wahl einer Basis) klar, daß im endlichdimensionalen Raum Bild ${ }_{\mathrm{R}} \psi$ dieser Wert $l$ gleichmäßig gewählt werden kann. Den Teilraum von $\bar{\Psi}_{0}$, dessen Elemente polynomialen Energieschranken genügen, notieren wir als $\bar{\Psi}_{0}^{P}$.

Die physikalische Bedeutung dieser Einschränkung ist folgende: In allen bisher konstruierten quantenfeldtheoretischen Modellenf erfüllen die Punktfelder solche Energieschranken [DF77]. Da wir vermuten, daß $\operatorname{Bild}_{\mathrm{R}} \psi$ in der interessierenden Situation aus solchen Punktfeldern besteht, erscheint eine derartige Einschränkung an das Hochenergieverhalten plausibel.

In der Literatur sind auch Feldtheorien bekannt, die nicht-polynomiales Hochenergieverhalten aufweisen [.Jaf67, Sum87], wobei allerdings die Temperiertheit der WightmanDistributionen aufgegeben werden muß. Wir werden solche Modelle hier nicht betrachten und unsere Analyse der Existenz lokaler Punktfelder nur unter der zusätzlichen Annahme

\footnotetext{
4 genauer in allen Modellen, die eine verschärfte Version der Osterwalder-Schrader-Axiome erfüllen
} 
polynomialer Hochenergieschranken ausführen. Tatsächlich geht diese Annahme aber nur an wenigen Stellen in die Argumentation ein, so daß viele Aspekte unabhängig von dieser Zusatzvoraussetzung betrachtet werden können.

Verhalten bei kleinen Wirkungen Wir benötigen noch einen quantitativen Begriff für das Verhalten der Abbildungen $\psi \in \bar{\Psi}$ im Bereich kleiner Wirkungen $E \cdot r$. Dazu definieren wir (analog zu heuristischen Motivation in Abschnitt 2.2) zunächst

$$
\|\psi\|_{E, r}:=\sup _{\sigma \in \Sigma(E)} \sup _{A \in \mathfrak{A}(r)} \frac{\left|\psi_{E, r}(\sigma, A)\right|}{\|\sigma\|\|A\|}
$$

wobei das Supremum nicht notwendigerweise endlich sein muß. Wie in Abschnitt 2.2 verwenden wir zur Analyse skaleninvariante Begriffe und betrachten daher für $w>0$

$$
\sup _{E \cdot r \leq w}\|\psi\|_{E, r}
$$

Dies wird ebenfalls eventuell $\infty$; diese technische Schwierigkeit umgehen wir durch Anwendung einer Funktion $B(x)=\frac{x}{1+x}$ mit der Konvention $B(\infty)=1$, die die Werte von (2.3.12) auf das Intervall $[0,1]$ „kontrahiert“. Wir wollen also

$$
f_{\psi}(w):=\sup _{E \cdot r \leq w} B\left(\|\psi\|_{E, r}\right)
$$

im Limes $w \rightarrow 0$ untersuchen. Hierzu definieren wir den „asymptotischen Exponenten“ von $f_{\psi}$ als

$$
\gamma\left(f_{\psi}\right):=\sup \left\{\lambda \mid w^{-\lambda} f_{\psi}(w) \underset{w \rightarrow 0}{\longrightarrow} 0\right\} \in[0, \infty]
$$

Es handelt sich hier also im Wesentlichen um den Exponenten, mit dem $f_{\psi}(w)$ für $w \rightarrow 0$ abfällt. (Details dazu findet man in Anhang 2.D.) Wir schreiben statt $\gamma\left(f_{\psi}\right)$ im folgenden einfach

$$
\gamma(\psi):=\gamma\left(f_{\psi}\right)
$$

Um auch hier die technische Schwierigkeit „unendlicher“ Werte zu umgehen, betrachten wir gelegentlich

$$
\delta(\psi):=\left\{\begin{array}{lll}
\frac{1}{1+\gamma(\psi)} & , & \gamma(\psi)<\infty \\
0 & , & \gamma(\psi)=\infty
\end{array}\right.
$$

Dann hat $\delta\left(\psi-\psi^{\prime}\right)$ die Eigenschaften einer Pseudometrik auf $\bar{\Psi}$ (siehe Anhang 2.D), liefert also einen vernünftigen Konvergenzbegriff.

\subsection{Ein Phasenraumkriterium}

Wir wollen nun eine allgemeine Theorie, die die grundlegenden Axiome der algebraischen Quantenfeldtheorie erfüllt, mit Hilfe der oben eingeführten Begriffe analysieren. 
Unsere Vorstellung ist, daß sich die lokale Meßauswertung $\Xi \in \bar{\Psi}$ durch Abbildungen von asymptotisch endlichem Rang approximieren läßt, und zwar um so besser, je höher deren Rang ist. Wir betrachten daher im allgemeinen Fall folgende Größen (für $n \in \mathbb{N}_{0}$ ):

$$
\gamma_{n}:=\max _{\psi_{(n)}} \gamma\left(\Xi-\psi_{(n)}\right) \in[0, \infty]
$$

wobei das Maximum über alle $\psi_{(n)} \in \bar{\Psi}_{0}$ mit $\operatorname{Rang} \psi_{(n)} \leq n$ genommen wird. Wie oben setzen wir

$$
\delta_{n}:=\frac{1}{1+\gamma_{n}} \in[0,1]
$$

Analog werden $\gamma_{n}^{P}$ und $\delta_{n}^{P}$ definiert, wobei das Maximum in diesem Fall nur über $\psi_{(n)} \in \bar{\Psi}_{0}^{P}$ genommen wird.

Es ist unmittelbar klar, daß $\gamma_{n} \geq \gamma_{n-1}$, daß die $\gamma_{n}$ also monoton wachsen und die $\delta_{n}$ monoton fallen. Weiterhin gilt stets

$$
\|\Xi\|_{E, r}=1 \forall E, r \quad \Rightarrow \quad \gamma_{0}=0, \delta_{0}=1 .
$$

Das weitere Verhalten der $\gamma_{n}, \delta_{n}$ respektive $\gamma_{n}^{P}, \delta_{n}^{P}$ charakterisiert die betrachtete Theorie. Wir können die auftretenden Fälle grob klassifizieren:

1. Es gilt $\delta_{n} \rightarrow 0$ für $n \rightarrow \infty$, jedoch $\delta_{n}>0 \forall n$. Dies entspricht dem in der heuristischen Motivation betrachteten Fall: Die Approximation wird mit zunehmendem Rang der Approximationsterme immer besser. Dieses Verhalten erwarten wir in allen Theorien, die aus Punktfeldern (ggf. mit polynomialen Energieschranken) aufgebaut sind. Konkret ist das in freien Feldtheorien mit endlich vielen Teilchensorten explizit der Fall (siehe Beispiel in Kapitel 7). Wir vermuten also beim beschriebenen Verhalten der $\delta_{n}$, daß die approximierenden Terme $\psi_{(n)}$ bzw. ihr Rechts-Bild aus Punktfeldern aufgebaut sind; es wird zu klären sein, inwieweit dies tatsächlich der Fall ist.

2. Es gilt $\delta_{n}=0$ für große $n$. Dies bedeutet, daß die Abbildung $\Xi$ beliebig gut (bzw. besser als jedes Polynom) durch nur endlich viele Rang-1-Terme approximiert werden kann. Solche Theorien besitzen also bei kleinem Er im Vergleich zur freien Feldtheorie eine Art „ausgedünnten Phasenraum“. Dies wird man bei Modellen erwarten, die nicht aus Punktfeldern aufgebaut sind, sondern aus anderen, auf endlich großen Gebieten lokalisierten Objekten. Solch ein Modell, in dem bereits ein einzelner Term $\psi_{(1)}=\omega \mathbf{1}$ ausreicht, um $\delta(\Xi-\omega \mathbf{1})=0 \mathrm{zu}$ erreichen, wurde von Lutz betrachtet [Lut.97] - siehe auch die Diskussion in Abschnitt 8.1.

Das Lutz'sche Modell ist für den beschriebenen Fall in folgendem Sinne generisch: Gibt es im Feldinhalt der Theorie (siehe Abschnitt 3.4) mindestens ein nichttriviales Quantenfeld, dann ist er tatsächlich unendlichdimensional, denn er muß auch alle Ableitungen dieses Feldes enthalten (Abschnitt 4.4). Đ Der Fall „, $\delta_{n}=0$ für große $n^{\prime \prime}$

\footnotetext{
5 Dies kann man folgendermaßen präzisieren: Unter sehr allgemeinen Bedingungen kann für lokale Punktfelder $\phi$ (außer dem Einsoperator) der Ausdruck $\phi(0) \Omega$ nicht beschränkt sein [BV95]. Nimmt man an, daß $\left\|R^{l} \phi(0) \Omega\right\|<\infty$ für geeignetes $l>0$, dann läßt sich unter Verwendung von $\partial_{t}=i[H, \cdot]$ zeigen, daß das Hochenergieverhalten von $\partial_{t}^{k} \phi(0) \Omega$ mit wachsendem $k$ divergenter wird; die Zeitableitungen des Feldes sind also nicht linear abhängig.
} 
führt aber, wie wir sehen werden, zu einem endlichdimensionalen Feldinhalt. Als Punktfelder werden also nur „klassische Observablen“ (Vielfache des Einsoperators) auftreten.

3. $\delta_{n} \geq \delta_{\infty}>0$ ist durch eine positive Konstante $\delta_{\infty}$ von unten beschränkt. Die Auswertung lokaler Messungen $\Xi$ kann also auch durch beliebig (aber endlich) viele Rang-1Terme nicht besser als $(E r)^{\delta_{\infty}}$ approximiert werden; der Phasenraum ist besonders "dicht gefüllt". Dies könnte folgende Ursachen haben:

- Es gibt in der Theorie unendlich viele Punktfelder der gleichen Energiedimension (beispielsweise in einer freien Feldtheorie mit unendlich vielen Teilchensorten). In diesem Fall reichen endlich viele Approximationsterme nicht aus, um den Anteil dieser Felder zu kompensieren.

- Im Fall $\delta_{n}^{P} \geq \delta_{\infty}^{P}>0$ könnte es sein, daß die zur Approximation benötigten Felder nicht polynomialen Energieschranken genügen.

Wir interessieren uns im folgenden für den Fall $\delta_{n} \rightarrow 0$, was den obigen Fall 1 und trivialerweise - auch 2 umfaßt. Dies läßt sich als Kriterium wie folgt formulieren:

Definition 2.1. Eine Theorie erfüllt das asymptotische Phasenraumkriterium [mit polynomialen Energieschranken], wenn es eine Folge $\left(\psi_{n}\right) \subset \bar{\Psi}_{0}\left[\left(\psi_{n}\right) \subset \bar{\Psi}_{0}^{P}\right]$ gibt, so daß

$$
\delta\left(\Xi-\psi_{n}\right) \rightarrow 0 .
$$

Das so formulierte Phasenraumkriterium sollte nach unserer heuristischen Motivation in der Lage sein, eine physikalisch interessante Klasse von Quantenfeldtheorien auszuzeichnen, nämlich solche mit einem „regulären Kurzabstandsverhalten“. Theorien dieser Art werden wir im folgenden betrachten. Wir werden in Kapitel [3 analysieren, inwieweit sich die Approximationsterme $\psi_{n}$ aus lokalen Punktfeldern zusammensetzen.

$\mathrm{Zu}$ fragen ist natürlich, ob das jetzt mathematisch präzisierte Kriterium tatsächlich in physikalisch relevanten Modellen streng erfüllt ist. Wir werden es in Kapitel [ zumindest in einer großen Klasse $e^{[}$freier Feldtheorien etablieren können. Mögliche Erweiterungen werden in Kapitel 8 diskutiert.

Es sei noch einmal darauf hingewiesen, daß das formulierte Kriterium nur darauf abzielt, Theorien mit einem wohldefinierten Punktfeldinhalt auszuzeichnen. Es ist nicht sensitiv dafür, zu entscheiden, inwiefern dieser Feldinhalt die Theorie vollständig bestimmt, inwiefern also sämtliche physikalischen Observablen im Verhalten des Systems bei kleinen Abständen codiert sind. Wie oben bereits erwähnt (Fall 2), kann es ohne weiteres vorkommen, daß das Kriterium in nichttrivialen Beispielen nur den Einsoperator als „Punktfeld“ liefert; solche Theorien lassen sich nicht aus ihrem Feldinhalt rekonstruieren. Wir werden dies in Abschnitt 8.2 näher ausführen.

\section{A Garbenstrukturen}

Im Rahmen unserer Analyse von Quantenfeldtheorien am Punkt betrachten wir oft Räume von normalen Zuständen mit einer Maximalenergie $E$ sowie die lokalen Algebren eines

\footnotetext{
${ }^{6}$ Probleme ergeben sich i.W. nur bei Theorien in niedrigen Raum-Zeit-Dimensionen.
} 
Doppelkegels $\mathcal{O}_{r}$. Ihre mathematische Struktur bei Variation von $E$ und $r$ soll hier näher untersucht werden.

Zunächst zur allgemeinen Situation: Die lokalen Algebren $\mathfrak{A}(r) \equiv \mathfrak{A}\left(\mathcal{O}_{r}\right)$ sind Unteralgebren von $\mathfrak{B}(\mathcal{H})$ für einen gewissen Vektorraum $\mathcal{H}$. Der mit $\Sigma$ bezeichnete Raum der normalen Funktionale über $\mathfrak{B}(\mathcal{H})$ ist identisch mit dem Prädualraum von $\mathfrak{B}(\mathcal{H})$; d.h. $\Sigma$ trägt eine Banachraumstruktur, deren Dualraum $\Sigma^{*}$ mit $\mathfrak{B}(\mathcal{H})$ übereinstimmt. Weiterhin ist $(P(E) \Sigma P(E))^{*}=P(E) \mathfrak{B}(\mathcal{H}) P(E)$. Wir werden im folgenden $\mathfrak{B}(\mathcal{H})$ und seine Unteralgebren meist mit der schwach-*-Topologie ausstatten (deren Dualitäten wir mit einem Stern unten kennzeichnen); es gilt dann $\mathfrak{B}(\mathcal{H})_{*}=\Sigma$ und $\mathfrak{A}(r)_{*}=\Sigma\lceil\mathfrak{A}(r)$.

Nun betrachten wir die $E$-Abhängigkeit der Räume $\Sigma(E)$ genauer. Offensichtlich gilt $\Sigma(E) \subset \Sigma\left(E^{\prime}\right)$ für $E \leq E^{\prime}$, so daß die Räume $\Sigma(E)$ mit den kanonischen Inklusionsabbildungen $\Sigma(E) \hookrightarrow \Sigma\left(E^{\prime}\right)$ zu einer Präkogarbe auf z.B. dem Intervall [1, $\left.\infty\right)$ werden; dieses Intervall wird gewählt, da wir uns lediglich für das Hochenergieverhalten der Theorie interessieren. Wir bezeichnen diese Präkogarbe mit $\bar{\Sigma}$.

(Der Begriff der Präkogarbe wird hier etwas anders als üblich verwendet. Üblicherweise würde man Räume $\Sigma(U)$ für alle offenen beschränkten Mengen $U \subset[1, \infty)$ und Inklusionen zwischen diesen betrachten. Indem wir aber statt $\Sigma(U)$ stets $\Sigma(E)$ mit $E=\sup U$ einsetzen, können wir uns auf die Angabe eines reellen Parameters beschränken.)

Ganz analog liefern die Algebren $\mathfrak{A}(r)$ mit den per Axiom geforderten Inklusionen $\mathfrak{A}(r) \hookrightarrow \mathfrak{A}\left(r^{\prime}\right)$ für $r<r^{\prime}$ eine Präkogarbe $\overline{\mathfrak{A}}$ auf $(0,1]$ - wir schränken durch diese Wahl unsere Analyse auf den Bereich kleiner $r$ ein.

Diese Strukturen übertragen sich auch auf die Dualräume der $\Sigma(E)$ und $\mathfrak{A}(r)$ : Wir erhalten Prägarben

$$
\bar{\Sigma}^{*}=\left\{\Sigma(E)^{*}\right\} \quad \text { und } \quad \overline{\mathfrak{A}}_{*}=\left\{\mathfrak{A}(r)_{*}\right\}
$$

auf den entsprechenden Intervallen; die Garbenabbildungen sind hierbei durch die gewöhnlichen Restriktionen gegeben.

Unser Formalismus zur Analyse von Punktfeldern verwendet bilineare Abbildungen $\Sigma(E) \times \mathfrak{A}(r) \rightarrow \mathbb{C}$. Genauer gesagt sollen diese Abbildungen für alle $E, r$ gegeben und verträglich sein mit den Inklusionen $\Sigma(E) \hookrightarrow \Sigma\left(E^{\prime}\right)$ und $\mathfrak{A}(r) \hookrightarrow \mathfrak{A}\left(r^{\prime}\right)$. Dazu bemerken wir, daß automatisch auch

$$
\bar{\Sigma} \times \overline{\mathfrak{A}}=\{\Sigma(E) \times \mathfrak{A}(r)\}
$$

zu einer Prägarbe auf $[1, \infty) \times(0,1]$ wird. (Hier ist statt einer offenen Menge $U \subset[1, \infty) \times$ $(0,1]$ wieder das Supremum der Menge in der jeweiligen Variablen einzusetzen.)

Die betrachteten Abbildungen $\psi$ sollen bilineare Formen auf dieser Präkogarbe sein, d.h. zu jedem Paar $(E, r)$ hat man eine bilineare Abbildung

$$
\psi_{E, r}: \Sigma(E) \times \mathfrak{A}(r) \rightarrow \mathbb{C}
$$


und diese $\psi_{E, r}$ sollen mit den Inklusionen $\Sigma(E) \times \mathfrak{A}(r) \hookrightarrow \Sigma\left(E^{\prime}\right) \times \mathfrak{A}\left(r^{\prime}\right)$ verträglich sein:[च

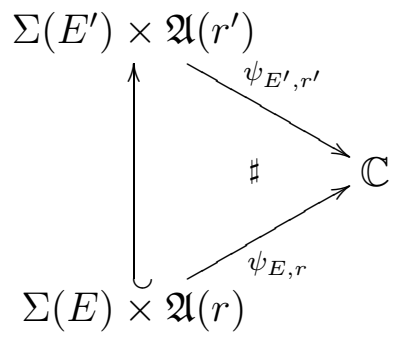

Außerdem sollen diese Abbildungen in einem gewissen Sinne regulär sein; das heißt, wir wollen Topologien auf den $\Sigma(E) \times \mathfrak{A}(r)$ einführen und fordern, daß die Abbildungen $\psi_{E, r}$ bezüglich dieser Topologien stetig sind. Es geht nun darum, diese Topologien geeignet zu definieren. Aus dem Kontext des zu entwickelnden Konstruktionsverfahrens heraus ergeben sich folgende Anforderungen:

1. In jedem Fall sollen folgende Bilinearformen zur Menge der stetigen Abbildungen gehören, da sie für die gewünschte Analyse benötigt werden:

$$
\begin{aligned}
\Xi:(\sigma, A) & \mapsto \sigma(A) \quad \text { und } \\
\psi_{A_{0} \sigma_{0}}:(\sigma, A) & \mapsto \sigma\left(A_{0}\right) \sigma_{0}(A)
\end{aligned}
$$

mit festem $\sigma_{0} \in \Sigma$ und $A_{0} \in \mathfrak{B}(\mathcal{H}) .8$

2. Umgekehrt wollen wir aus der Stetigkeit einer Rang-1-Abbildung der obigen Form $\psi_{A_{0} \sigma_{0}} \neq 0$ (oder kurz $A_{0} \sigma_{0} \neq 0$ ) folgern können, daß $\sigma_{0}$ schwach-*-stetig auf jedem $\mathfrak{A}(r)$ und $A_{0}$ normstetig auf jedem $\Sigma(E)$ ist. Diese Eigenschaft erreicht man gerade, indem man fordert, daß die Abbildungen

$$
\begin{array}{rlll}
m_{\mathrm{L}, \sigma}: & \mathfrak{A}(r) \rightarrow \Sigma(E) \times \mathfrak{A}(r), & A \mapsto(\sigma, A) & (\sigma \in \Sigma(E) \text { fest }), \\
m_{\mathrm{R}, A}: & \Sigma(E) \rightarrow \Sigma(E) \times \mathfrak{A}(r), & \sigma \mapsto(\sigma, A) & (A \in \mathfrak{A}(r) \text { fest })
\end{array}
$$

stetig sind, wenn man $\Sigma(E)$ mit der Normtopologie und $\mathfrak{A}(r)$ mit der schwach-*Topologie versieht. (Dann wird $\psi_{A_{0} \sigma_{0}} \circ m_{\mathrm{L}, \sigma}=\sigma\left(A_{0}\right) \sigma_{0}$ stetig, und folglich ist $\sigma_{0}$ schwach-*-stetig; analoges gilt für $m_{\mathrm{R}, A}$.)

Die zu wählende Topologie auf $\Sigma(E) \times \mathfrak{A}(r)$ sollte beide Anforderungen 1 und 2 erfüllen. Man stellt allerdings fest, daß die „üblichen“ Topologien diesen Anforderungen nicht genügen: So scheint es natürlich, $\Sigma(E) \times \mathfrak{A}(r)$ mit dem Produkt der beiden Normtopologien auf $\Sigma(E)$ und $\mathfrak{A}(r)$ zu versehen; doch läßt sich damit die schwach-*-Stetigkeit der Funktionale $\sigma_{0}$ nicht sicherstellen. (Wir werden die schwach-*-Stetigkeit später benötigen, um Fortsetzungen gewisser Funktionale mit Hilfe des Satzes von Hahn-Banach zu erhalten.) Verwendet man andererseits das Produkt aus Normtopologie auf $\Sigma(E)$ und schwach-*-Topologie auf $\mathfrak{A}(r)$, dann wird die Abbildung $\Xi$ nicht stetig: Wenn $\sigma \rightarrow 0$ in der Normtopologie und $A \rightarrow 0$ im schwach-*-Sinn, dann folgt im allgemeinen nicht, daß $\sigma(A) \rightarrow 0$.

Allerdings bieten sich aus den beiden Forderungen heraus die folgenden zwei Topologien unmittelbar an:

\footnotetext{
7 Zur Notation kommutativer Diagramme siehe Seite 201.

8 Tatsächlich benötigen wir diese Abbildungen noch für allgemeinere $A_{0}$; es reicht aber aus, hier als schwächere Bedingung die Stetigkeit für $A_{0} \in \mathfrak{B}(\mathcal{H})$ zu fordern.
} 
1. Man betrachtet die initiale Topologie $\tau_{i}$ auf $\Sigma(E) \times \mathfrak{A}(r)$ bezüglich aller Abbildungen $\Xi$ und $\psi_{A_{0} \sigma_{0}}$ mit $\sigma_{0} \in \Sigma$ und $A_{0} \in \mathfrak{B}(\mathcal{H})$. In dieser Topologie sind alle diese Abbildungen stetig (Forderung 11 ist also erfüllt), und sie ist die gröbste aller Topologien mit dieser Eigenschaft.

2. Man verwendet die finale Topologie $\tau_{f}$ bezüglich aller Abbildungen $m_{\mathrm{L}, \sigma}$ und $m_{\mathrm{R}, A}$. In dieser Topologie sind die $m_{\mathrm{L}, \sigma}$ und $m_{\mathrm{R}, A}$ stetig (sie genügt also Forderung 2), und $\tau_{f}$ ist die feinste aller Topologien, die dies erfüllen.

Wir zeigen nun, daß sowohl $\tau_{f}$ wie $\tau_{i}$ tatsächlich beide Anforderungen erfüllen. Die Abbildungen $m_{\mathrm{L}, \sigma}$ sind nämlich auch $\tau_{i}$-stetig: Dazu ist zu zeigen, daß die Kompositionen $\Xi \circ m_{\mathrm{L}, \sigma}$ und $\psi_{A_{0} \sigma_{0}} \circ m_{\mathrm{L}, \sigma}$ stetig sind. Man hat für $A \in \mathfrak{A}(r)$

$$
\Xi \circ m_{\mathrm{L}, \sigma}(A)=\Xi(\sigma, A)=\sigma(A)
$$

was bei festem $\sigma$ sicher stetig in $A$ ist, und

$$
\psi_{A_{0} \sigma_{0}} \circ m_{\mathrm{L}, \sigma}(A)=\psi_{A_{0} \sigma_{0}}(\sigma, A)=\sigma\left(A_{0}\right) \sigma_{0}(A)
$$

was ebenfalls in $A$ stetig ist. Also ist $m_{\mathrm{L}, \sigma}$ stetig bezüglich $\tau_{i}$, und ebenso sind es die $m_{\mathrm{R}, A}$; daher erfüllt $\tau_{i}$ auch Forderung 2, und es gilt

$$
\tau_{i} \subset \tau_{f}
$$

Hieraus folgt außerdem, daß $\tau_{f}$ auch Forderung 1 erfüllt (sie ist feiner als $\tau_{i}$ ).

Damit sind die für unsere Zwecke geeigneten Topologien vollständig charakterisiert: Eine Topologie $\tau$ auf $\Sigma(E) \times \mathfrak{A}(r)$ erfüllt die Forderungen 1 und 2 genau dann, wenn sie feiner als $\tau_{i}$ und gröber als $\tau_{f}$ ist, also wenn

$$
\tau_{i} \subset \tau \subset \tau_{f}
$$

Für unsere Zwecke erscheint es natürlicher, mit $\tau_{f}$ zu arbeiten, und wir werden im folgenden stets annehmen, daß $\Sigma(E) \times \mathfrak{A}(r)$ mit der Topologie $\tau_{f}$ versehen ist.

Wir bemerken noch, daß $\tau_{f}$ auch mit der Kogarbenstruktur auf $\bar{\Sigma} \times \overline{\mathfrak{A}}$ verträglich ist: Für $E<E^{\prime}, r<r^{\prime}$ liest man aus den kommutativen Diagrammen
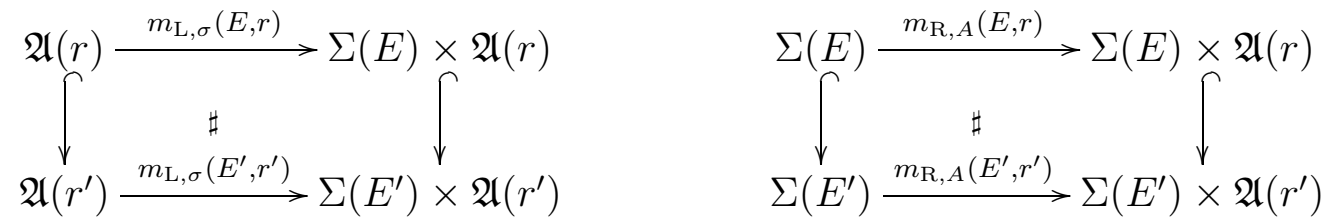

leicht ab, daß die Inklusionen $\Sigma(E) \times \mathfrak{A}(r) \hookrightarrow \Sigma\left(E^{\prime}\right) \times \mathfrak{A}\left(r^{\prime}\right)$ stetige Abbildungen sind (denn ihre Kompositionen mit den $m_{\mathrm{L}, \sigma}$ und $m_{\mathrm{R}, A}$ sind es). Insofern können wir sagen, daß $\tau_{f}$ eine Topologie auf der Präkogarbe $\bar{\Sigma} \times \overline{\mathfrak{A}}$ liefert. 9

Wir werden im folgenden mit $\bar{\Psi}$ die Menge aller bilinearen, $\tau_{f}$-stetigen Abbildungen auf $\bar{\Sigma} \times \overline{\mathfrak{A}}$ bezeichnen.

\footnotetext{
${ }^{9}$ Für $\tau_{i}$ wäre dies allerdings auch erfüllt.
} 
Es gilt nun, die Elemente $\psi \in \bar{\Psi}$ weiter zu analysieren, und insbesondere den Spezialfall einer Abbildung von endlichem Rang zu formulieren. Dies werden wir zunächst für festes $E$ und $r$ tun, d.h. wir werden die $\tau_{f}$-stetigen Abbildungen

$$
\psi_{E, r}: \Sigma(E) \times \mathfrak{A}(r) \rightarrow \mathbb{C}
$$

betrachten. Es sei daran erinnert, daß hier $\Sigma(E)$ stets mit der Normtopologie und $\mathfrak{A}(r)$ mit der schwach-*-Topologie versehen ist.

Es lohnt sich, die Analyse der Abbildungen von endlichem Rang etwas allgemeiner durchzuführen, was im nächsten Abschnitt geschehen soll.

\section{B Bilinearformen von endlichem Rang}

Im folgenden seien $U, V$ zwei hausdorffsche, lokal konvexe topologische Vektorräume mit Topologien $\tau_{U}, \tau_{V}$. (Wir werden die Dualräume usw. bezüglich $\tau_{U}$ mit dem Symbol * kennzeichnen, die bezüglich $\tau_{V}$ mit ${ }_{*}$.) Unser Ziel ist es, bilineare Abbildungen $\psi: U \times V \rightarrow$ $\mathbb{C}$ zu analysieren, die gewissen Stetigkeitsbedingungen genügen. Wir definieren dazu die Abbildungen

$$
\begin{aligned}
& m_{\mathrm{L}, u}: \quad V \rightarrow U \times V, \quad v \mapsto(u, v) \quad(u \in U \text { fest }), \\
& m_{\mathrm{R}, v}: \quad U \rightarrow U \times V, \quad u \mapsto(u, v) \quad(v \in V \text { fest })
\end{aligned}
$$

und versehen $U \times V$ mit der finalen Topologie bezüglich aller dieser Abbildungen. Mit $\Psi$ bezeichnen wir die Menge aller hierin stetigen, bilinearen Abbildungen $U \times V \rightarrow \mathbb{C}$.

Zur Analyse der Abbildungen in $\Psi$ führen wir sie zunächst auf lineare Abbildungen zurück. $\mathrm{Zu} \psi \in \Psi$ definieren wir dazu die Linksadjungierte $\psi_{\mathrm{L}}$ :

$$
\psi_{\mathrm{L}}: U \rightarrow V_{*}, \quad u \mapsto \psi(u, \cdot) .
$$

Das ist wohldefiniert, d.h. die Bilder $\psi_{\mathrm{L}}(u)$ sind stetige Funktionale auf $V$ : Man hat nämlich $\psi_{\mathrm{L}}(u)=\psi \circ m_{\mathrm{L}, u}$, und beide Abbildungen auf der rechten Seite sind stetig.

Wir versehen $V_{*}$ mit der von $V$ induzierten schwachen Topologie, so daß $\left(V_{*}\right)^{*}=V$ wird. $\square$ Dann ist $\psi_{\mathrm{L}}$ nicht nur linear, sondern auch stetig: Man hat für $v \in V$

$$
\left|\left(\psi_{\mathrm{L}}(u)\right)(v)\right|=|\psi(u, v)|=\left|\psi \circ m_{\mathrm{R}, v}(u)\right| \rightarrow 0
$$

für $u \rightarrow 0$, da $m_{\mathrm{R}, v}$ stetig ist.

Ebenso erhält man die Rechtsadjungierte $\psi_{\mathrm{R}}$ als stetige, lineare Abbildung

$$
\psi_{\mathrm{R}}: V \rightarrow U^{*}, \quad v \mapsto \psi(\cdot, v),
$$

wobei $U^{*}$ analog mit der von $U$ gelieferten schwachen Topologie versehen ist, so daß $\left(U^{*}\right)_{*}=$ $U$.

Die beiden adjungierten Abbildungen $\psi_{\mathrm{L}}$ und $\psi_{\mathrm{R}}$ hängen sehr eng zusammen; es ist nämlich $\psi_{\mathrm{L}}^{*}=\psi_{\mathrm{R}}$ und $\psi_{\mathrm{R} *}=\psi_{\mathrm{L}}$. Man sieht dies leicht durch eine kurze Rechnung: Für $v \in V=V_{*}^{*}$ und $u \in U$ ist

$$
\begin{aligned}
& \left(\psi_{\mathrm{L}}^{*} v\right)(u)=v\left(\psi_{\mathrm{L}} u\right)=v(\psi(u, \cdot))=\psi(u, v) ; \\
& \left(\psi_{\mathrm{R}} v\right)(u)=(\psi(\cdot, v))(u)=\psi(u, v) \quad \Rightarrow \quad \psi_{\mathrm{L}}^{*}=\psi_{\mathrm{R}} .
\end{aligned}
$$

\footnotetext{
${ }^{10}$ Die „Dualitäten“ bzgl. der Topologie auf $V_{*}$ bezeichnen wir mit*.
} 
Analog folgt $\psi_{\mathrm{R} *}=\psi_{\mathrm{L}}$. Wir erhalten also folgendes Bild:

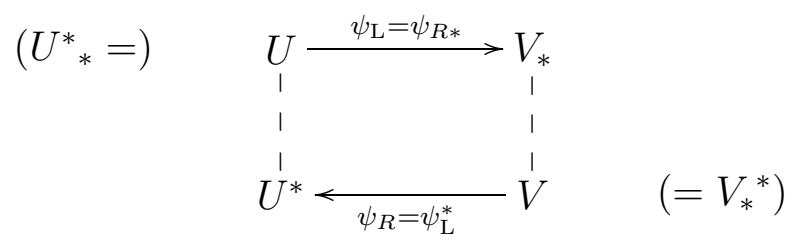

Weiter interessieren wir und für Kern und Bild von $\psi_{\mathrm{L}}$ und $\psi_{\mathrm{R}}$. Wir nennen

$$
\operatorname{Bild}_{\mathrm{L}} \psi:=\operatorname{Bild} \psi_{\mathrm{L}} \subset V_{*} \quad \text { und } \quad \operatorname{Kern}_{\mathrm{L}} \psi:=\operatorname{Kern} \psi_{\mathrm{L}} \subset U
$$

das Links-Bild respektive den Links-Kern von $\psi$; analog werden das Rechts-Bild und der Rechts-Kern definiert. Man hat unmittelbar das kommutative Diagramm

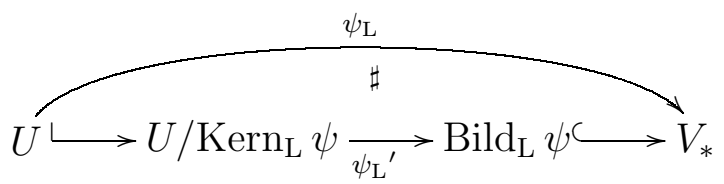

wobei $\psi_{\mathrm{L}}{ }^{\prime}=\psi_{\mathrm{L}} / \operatorname{Kern}_{\mathrm{L}} \psi$ stetig wird, wenn man $U / \operatorname{Kern}_{\mathrm{L}} \psi$ mit der Quotiententopologie und $\operatorname{Bild}_{\mathrm{L}} \psi$ mit der Teilraum-Topologie versieht. Die bijektive Abbildung $\psi_{\mathrm{L}}{ }^{\prime}$ werden wir, wenn keine Verwechslungsmöglichkeit besteht, meist auch mit $\psi_{\mathrm{L}}$ oder sogar einfach mit $\psi$ bezeichnen (es handelt sich um dieselbe Abbildung, die nur „zwischen anderen Räumen“ definiert ist).

Ganz analog erhält man für $\psi_{\mathrm{R}}$ :

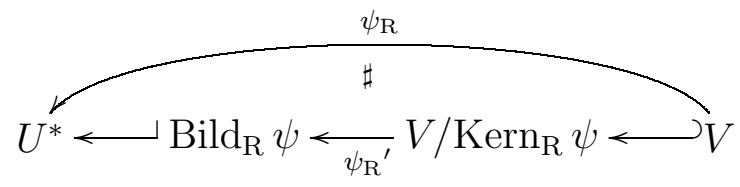

Wir wollen die beiden Diagramme (2.B.8) und (2.B.9) nun zusammenfassen und zeigen, daß $\operatorname{Bild}_{\mathrm{L}} \psi=\left(V / \operatorname{Kern}_{\mathrm{R}} \psi\right)_{*}$. (V/Kern $\mathrm{R} \psi$ trägt hierbei wieder die Quotiententopologie.)

Zunächst macht man sich klar, daß die Linearformen $v_{*} \in \operatorname{Bild}_{\mathrm{L}} \psi$ auf dem Quotientenraum $V / \operatorname{Kern}_{\mathrm{R}} \psi$ wohldefiniert sind: Es gilt $v_{*}=\psi_{\mathrm{L}} u$ mit gewissem $u \in U$, und für $v \in \operatorname{Kern}_{\mathrm{R}} \psi$ gilt dann

$$
v_{*}(v)=\psi_{\mathrm{L}} u(v)=\psi(u, v)=\underbrace{\psi_{\mathrm{R}} v}_{=0}(u)=0 .
$$

Wir können also Restklassen aus $V / \operatorname{Kern}_{\mathrm{R}} \psi$ in Linearformen aus $\operatorname{Bild}_{\mathrm{L}} \psi$ einsetzen, oder mit anderen Worten: $\left(\operatorname{Bild}_{\mathrm{L}} \psi, V / \operatorname{Kern}_{\mathrm{R}} \psi\right)$ ist ein Bilinearsystem bzgl. der Einsetzung. Es handelt sich sogar um ein Dualsystem, d.h. die Einsetzung ist „definit": Für festes $v_{*} \in \operatorname{Bild}_{\mathrm{L}} \psi$ folgt

$$
v_{*}\left(v+\operatorname{Kern}_{\mathrm{R}} \psi\right)=0 \quad \forall v \in V / \operatorname{Kern}_{\mathrm{R}} \psi \quad \Rightarrow \quad v_{*}=0
$$


für festes $v+\operatorname{Kern}_{\mathrm{R}} \psi \in V / \operatorname{Kern}_{\mathrm{R}} \psi$ gilt

$$
\begin{aligned}
& v_{*}\left(v+\operatorname{Kern}_{\mathrm{R}} \psi\right)=0 \\
& \Rightarrow \quad \psi_{\mathrm{L}} u\left(v+\operatorname{Kern}_{\mathrm{R}} \psi\right)=0 \\
& \forall v_{*} \in \operatorname{Bild}_{\mathrm{L}} \psi \\
& \forall u \in U \\
& \Rightarrow u(\psi_{\mathrm{R}} v+\underbrace{\psi_{\mathrm{R}} \operatorname{Kern}_{\mathrm{R}} \psi}_{=0})=0 \\
& \forall u \in U \\
& \Rightarrow \quad \psi_{\mathrm{R}} v=0 \text { in } U^{*} \\
& \Rightarrow \quad v \in \operatorname{Kern}_{\mathrm{R}} \psi \quad \text { bzw. } v=0 \text { in } V / \operatorname{Kern}_{\mathrm{R}} \psi \text {. }
\end{aligned}
$$

$\operatorname{Bild}_{\mathrm{L}} \psi$ ist zunächst mit der Relativtopologie versehen (induziert von der schwachen Topologie auf $V_{*}$ ); man sieht durch Betrachtung der definierenden Halbnormen aber leicht, daß diese mit der von $V / \operatorname{Kern}_{\mathrm{R}} \psi$ gelieferten schwachen Topologie übereinstimmt. Damit ist wegen der Dualsystem-Eigenschaft klar, daß in dieser Topologie

$$
\left(\operatorname{Bild}_{\mathrm{L}} \psi\right)^{*}=V / \operatorname{Kern}_{\mathrm{R}} \psi
$$

Ganz analog zeigt man, daß

$$
\left(\operatorname{Bild}_{\mathrm{R}} \psi\right)_{*}=V / \operatorname{Kern}_{\mathrm{L}} \psi .
$$

Wir erhalten damit folgendes Diagramm von dualen Räumen und Abbildungen:

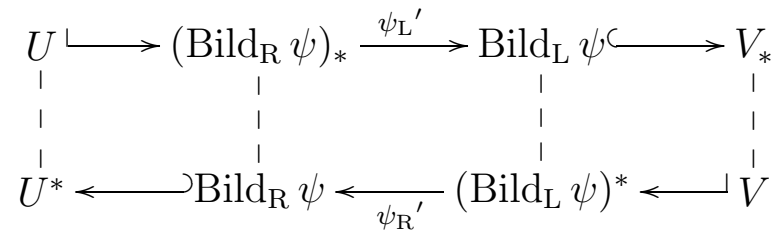

Es ist allerdings im allgemeinen nicht bekannt, daß $\left(\operatorname{Bild}_{\mathrm{R}} \psi\right)_{*}{ }^{*}=\operatorname{Bild}_{\mathrm{R}} \psi$ usw. Dies ist nur im unten betrachteten Spezialfall gesichert.

Wir interessieren uns nun besonders für den Fall, daß einer der Räume Bild $_{\mathrm{L}} \psi$ und $\operatorname{Bild}_{\mathrm{R}} \psi$ endlichdimensional ist. Als Teilraum eines hausdorffschen, lokal konvexen topologischen Vektorraums trägt er dann die Standardtopologie. Da $\psi_{\mathrm{L}}^{\prime}$ und $\psi_{\mathrm{R}}{ }^{\prime}$ Isomorphismen sind, ist sofort klar, daß dann $\operatorname{Bild}_{\mathrm{L}} \psi, \operatorname{Bild}_{\mathrm{R}} \psi$ sowie die zugehörigen Dualräume alle endlichdimensional sind und dieselbe Dimension besitzen.

Wir sagen in diesem Fall, daß die bilineare Abbildung $\psi$ von endlichem Rang ist und definieren

$$
\operatorname{Rang} \psi:=\operatorname{dim} \operatorname{Bild}_{\mathrm{L}} \psi=\operatorname{dim} \operatorname{Bild}_{\mathrm{R}} \psi
$$

Dieser Typ von Abbildungen ist besonders einfach, da er sich auf die Abbildungen $\psi_{\mathrm{L}}{ }^{\prime}$ bzw. $\psi_{\mathrm{R}}{ }^{\prime}$ zwischen endlichdimensionalen Räumen reduzieren läßt. (Durch Wahl geeigneter Basen in $\left(\operatorname{Bild}_{\mathrm{L}} \psi\right)_{*}$ und $\operatorname{Bild}_{\mathrm{R}} \psi$ können wir erreichen, daß $\psi_{\mathrm{L}}$ durch die Diagonalmatrix $\operatorname{diag}(1, \ldots, 1)$ beschrieben wird.)

Ein wichtiger Spezialfall ist der einer Rang-1-Abbildung $\psi$ : In diesem Fall sind $\left(\text { Bild }_{\mathrm{R}} \psi\right)_{*}$ und $\operatorname{Bild}_{\mathrm{L}} \psi$ eindimensional; die Abbildung $\psi_{\mathrm{L}}$ ist zwischen diesen Räumen nur die Multiplikation mit einem Skalar, durch geeignete Basiswahl mit dem Skalar 1. Bezeichnen wir die kanonische Projektion $U \longrightarrow\left(\operatorname{Bild}_{\mathrm{R}} \psi\right)_{*} \cong \mathbb{C}$ als $u^{*}$ (sie ist eine stetige Linearform, 
also $u^{*} \in U^{*}$ ) und ein dazu passend gewähltes Basiselement in $\operatorname{Bild}_{\mathrm{L}} \psi$ als $v_{*} \in V_{*}$, dann erhalten wir also

$$
\psi_{\mathrm{L}}=u^{*} v_{*} \quad \text { bzw. } \quad \psi=u^{*} v_{*}
$$

in dem Sinne, daß $\psi(u, v)=u^{*}(u) v_{*}(v)$ und $\psi_{\mathrm{L}}(u)=u^{*}(u) v_{*}$.

Wir interessieren uns weiterhin für eine Zerlegung von Bilinearformen von endlichem Rang in solche von kleinerem Rang. Dazu sei $\psi \in \Psi$ vom Rang $n$. Gilt für Abbildungen $\psi_{1}, \ldots, \psi_{k} \in \Psi$ vom Rang $n_{1}, \ldots, n_{k}$ die Gleichung

$$
\psi=\psi_{1}+\ldots+\psi_{k}, \quad n=n_{1}+\ldots+n_{k}
$$

so nennen wir dies eine (direkte) Zerlegung von $\psi$. Aus Gründen, die weiter unten ausgeführt werden, schreiben wir oft auch $\psi=\psi_{1} \oplus \ldots \oplus \psi_{k}$. Der Einfachheit halber werden wir im folgenden direkte Zerlegungen von $\psi$ in nur zwei Abbildungen genauer betrachten; für Zerlegungen in mehr als 2 Abbildungen gilt aber entsprechendes.

$\mathrm{Zu}$ einer gegebenen direkten Zerlegung $\psi=\psi_{1}+\psi_{2}$ (und damit $\psi_{L}=\psi_{1, L}+\psi_{2, L}$ ) gehört offenbar eine Zerlegung $\operatorname{Bild}_{\mathrm{L}} \psi=\operatorname{Bild}_{\mathrm{L}} \psi_{1} \oplus \operatorname{Bild}_{\mathrm{L}} \psi_{2}$ als direkte Summe. Analoges gilt für $\operatorname{Bild}_{\mathrm{R}} \psi$. Die Inklusionsabbildungen $\operatorname{Bild}_{\mathrm{R}} \psi_{j} \hookrightarrow \operatorname{Bild}_{\mathrm{R}} \psi$, die das Diagramm

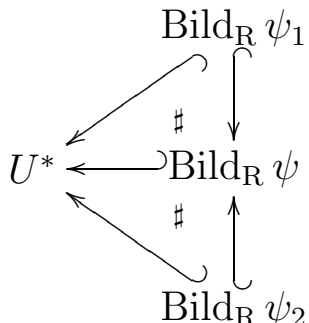

kommutativ machen, liefern Projektionen zwischen den zugehörigen Dualräumen, so daß

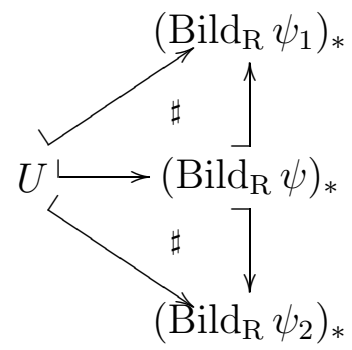

kommutiert, was bedeutet, daß $\psi_{1, \mathrm{~L}}$ und $\psi_{2, \mathrm{~L}}$ auf $\left(\operatorname{Bild}_{\mathrm{R}} \psi\right)_{*}$ wohldefiniert sind. Wir können diese „Aufteilung“ von $\left(\text { Bild }_{\mathrm{R}} \psi\right)_{*}$ noch verfeinern: Aus Dimensionsgründen ist $\left(\operatorname{Bild}_{\mathrm{R}} \psi\right)_{*}=$ $\operatorname{Kern}_{\mathrm{L}} \psi_{1} \oplus \operatorname{Kern}_{\mathrm{L}} \psi_{2}$, und $\psi_{1, \mathrm{~L}}$ wirkt dabei nur auf $\operatorname{Kern}_{\mathrm{L}} \psi_{2}$ usw., so daß bezüglich dieser Zerlegung gilt $\psi=\psi_{1} \oplus \psi_{2}$. Die Inklusionen in dieser direkten Summe liefern Projektionen Bild $_{\mathrm{R}} \psi \longleftrightarrow \operatorname{Bild}_{\mathrm{R}} \psi_{j}$ in den Dualräumen, und entsprechendes mit vertauschtem L und R. 
Wir erhalten dann insgesamt das folgende Diagramm:

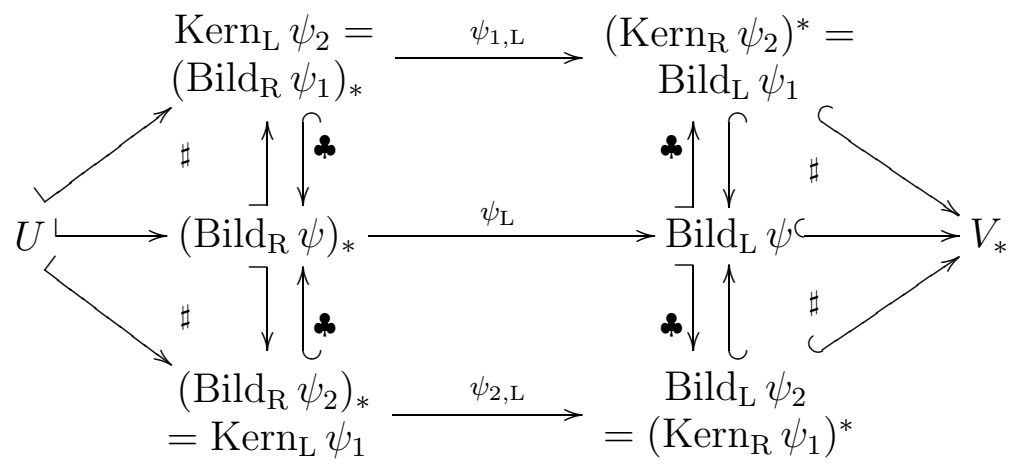

Hierzu sei bemerkt, daß zwar die äußeren Dreiecke kommutativ sind, daß die mit os bezeichneten Abbildungen die Vierecke in der Mitte jedoch im allgemeinen nicht kommutativ machen; diese Abbildungen hängen außerdem nicht nur von einer einzelnen zugehörigen Bilinearform $\psi_{j, \mathrm{~L}}$, sondern stets von der gesamten Aufspaltung ab.

Ein analoges Bild ergibt sich zwischen den Dualräumen bzw. für die duale Abbildung $\psi_{\mathrm{R}}=\psi_{1, \mathrm{R}} \oplus \psi_{2, \mathrm{R}}$.

Auf diese Weise führt uns eine direkte Zerlegung $\psi=\psi_{1}+\psi_{2}$ zu einer (eindeutig bestimmten) Zerlegung der Räume $\operatorname{Bild}_{\mathrm{L}} \psi, \operatorname{Bild}_{\mathrm{R}} \psi$ und ihrer Dualräume in direkte Summen, so daß $\psi=\psi_{1} \oplus \psi_{2}$.

Umgekehrt induziert z.B. eine gegebene Zerlegung $\operatorname{Bild}_{\mathrm{L}} \psi=V_{1} \oplus V_{2}$ sofort eine Zerlegung der anderen drei Räume und eine direkte Zerlegung der Abbildung $\psi$ in $\psi_{1} \oplus \psi_{2}$, so daß wieder das Diagramm (2.B.21) gilt. Analoges gilt, wenn man von einer Zerlegung eines beliebigen anderen der endlichdimensionalen Räume startet.

Es ist zweckmäßig, diesen Fall noch weiter $\mathrm{zu}$ formalisieren: $\mathrm{Zu} \operatorname{Bild}_{\mathrm{L}} \psi=V_{1} \oplus V_{2}$ sei $W_{1} \oplus W_{2}$ die zugehörige Zerlegung von $\operatorname{Bild}_{\mathrm{R}} \psi$. Anhand der direkten Zerlegung von $\psi$ rechnet man leicht nach, daß für $\sigma \in \bar{\Sigma}$ folgende Äquivalenz gilt:

$$
\psi(\sigma, \cdot) \in V_{1} \quad \Leftrightarrow \quad \sigma\left\lceil W_{2}=0\right.
$$

Der Raum $W_{2}$ ist also durch Angabe von $V_{1}$ eindeutig bestimmt (und umgekehrt), unabhängig von der Wahl von $V_{2}$ bzw. $W_{1}$. Wir nennen daher $W_{2}$ den adjungierten Kern $\square$ von $V_{1}$. Analog ist $W_{1}$ der adjungierte Kern von $V_{2}$.

Mit den obigen Ergebnissen ist es insbesondere möglich, eine Rang- $n$-Abbildung $\psi \in$ $\Psi$ in eine direkte Summe von Rang-1-Abbildungen aufzuteilen: Jede Wahl einer Basis $\left\{v_{* 1}, \ldots, v_{* n}\right\}$ von $\operatorname{Bild}_{\mathrm{L}} \psi$ entspricht nämlich eine Zerlegung von $\operatorname{Bild}_{\mathrm{L}} \psi$ in eine Summe eindimensionaler Räume und liefert damit nach obigen Ergebnissen

$$
\psi=u_{1}^{*} v_{* 1} \oplus \ldots \oplus u_{n}^{*} v_{* n}
$$

mit linear unabhängigen $u_{1}^{*}, \ldots, u_{n}^{*} \in U^{*}$ (einer Basis von $\operatorname{Bild}_{\mathrm{R}} \psi$ ), welche durch die Vorgabe der $v_{* 1}, \ldots, v_{* n}$ eindeutig bestimmt sind.

\footnotetext{
${ }^{11}$ Diese Begriffsbildung bezieht sich immer auf eine festgehaltene bilineare Abbildung $\psi$.
} 


\section{C Abbildungen von asymptotisch endlichem Rang}

Wir kehren nun zu dem uns interessierenden Spezialfall $U=\Sigma(E), V=\mathfrak{A}(r)$ zurück und betrachten wieder bilineare Abbildungen auf der Präkogarbe $\bar{\Sigma} \times \overline{\mathfrak{A}}$.

Bisher haben wir geklärt, was wir bei festem $E$ und $r$ unter einer Abbildung von endlichem Rang verstehen und wie sie zu analysieren ist. Für die Analyse von Punktfeldern interessiert uns jedoch der Grenzwert „verschwindenden Phasenraumvolumens" $E \cdot r \rightarrow 0$; es ist zu präzisieren, was in diesem Fall die interessierenden Abbildungen „von endlichem Rang" sind.

Man könnte hier sicherlich fordern, daß z.B.

$$
\limsup _{E r \rightarrow 0} \operatorname{Rang} \psi_{E, r}<\infty,
$$

daß also der Rang von $\psi_{E, r}$ im Grenzfall gleichmäßig beschränkt bleibt, doch wäre dies für unsere Analyse zu schwach; es schließt nicht aus, daß der Rang für $E r \rightarrow 0$ oszilliert. Zwar ließe sich durch Übergang zu einer geeigneten diskreten Folge $\left(E_{n}, r_{n}\right)$ erreichen, daß sogar Rang $\psi_{E_{n}, r_{n}}=$ const. für große $n$; wir sind jedoch nicht so sehr an der numerischen Größe des Rangs interessiert, sondern am (Links-/Rechts-)Bild von $\psi_{E, r}$ als Vektorraum. Um ihn im Limes $E r \rightarrow 0$ analysieren zu können, verlangen wir, daß nicht nur die Dimension des Bildes, sondern das Bild selbst für kleine $E r$ „konstant“ ist. Dabei ist „konstant“ als „mit den Garbenabbildungen verträglich" zu verstehen.

Wir wollen dies etwas genauer ausführen. Es sei dazu $\psi \in \bar{\Psi}$ so, daß alle $\psi_{E, r}$ vom gleichen endlichen Rang sind, und $E^{\prime} \geq E, r^{\prime} \geq r$ seien fest gegeben. Dann kommutiert das Diagramm

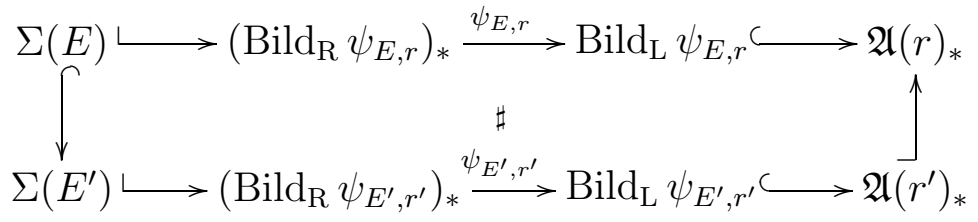

wegen der Verträglichkeit von $\psi$ mit den Garbenstrukturen. Das Bild der Abbildung 우우웅ㅇㅇ können wir wegen der Kommutativität von (2.C.2) durch die Inklusion $\operatorname{Bild}_{\mathrm{L}} \psi_{E, r} \hookrightarrow \mathfrak{A}(r)_{*}$ hindurch „zurückziehen“ und erhalten damit eine aus Dimensionsgründen bijektive Abbildung $\operatorname{Bild}_{\mathrm{L}} \psi_{E^{\prime}, r^{\prime}} \longleftrightarrow \operatorname{Bild}_{\mathrm{L}} \psi_{E, r}$, so daß das Diagramm

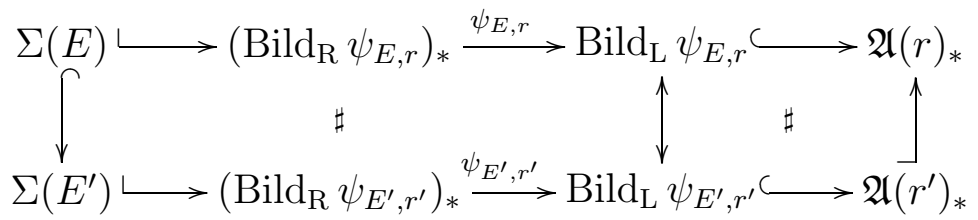

kommutiert. Analog erhalten wir auf der dualen Seite eine Bijektion $\operatorname{Bild}_{\mathrm{R}} \psi_{E^{\prime}, r^{\prime}} \longleftrightarrow$ Bild $_{\mathrm{R}} \psi_{E, r}$, deren duale Abbildung das Diagramm

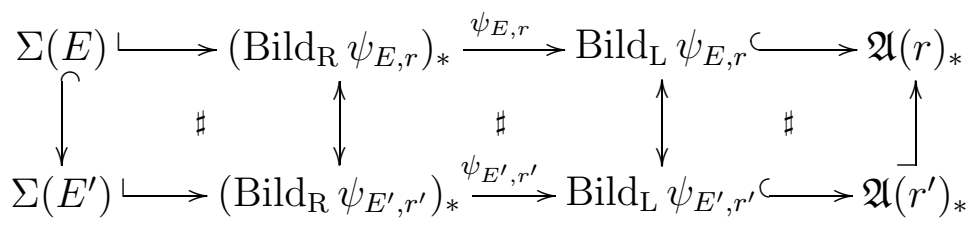


kommutativ ergänzt. In diesem Sinne sind das Links- und das Rechts-Bild von $\psi$ dann konstant.

Allerdings gilt obige Analyse nur für den Fall $E^{\prime} \geq E$ und $r^{\prime} \geq r$. Dieser Fall ist jedoch im Grenzwert $E r \rightarrow 0$ eher irrelevant, typischerweise hat man $r \rightarrow 0, E \rightarrow \infty$, also z.B. $E^{\prime} \geq E$ und $r^{\prime} \leq r$. In diesem Fall läßt sich also die „Konstanz" der Bilder nicht wie oben folgern, sondern wir müssen sie explizit fordern:

Definition 2.2. Eine Abbildung $\psi \in \bar{\Psi}$ heiße Abbildung von asymptotisch endlichem Rang, wenn für kleine $E \cdot r, E^{\prime} \cdot r^{\prime}$ folgende Bedingungen erfüllt sind:

- $\psi_{E, r}$ ist von endlichem Rang;

- Zu E, $E^{\prime}, r, r^{\prime}$ existieren Isomorphismen $j_{L}$ und $j_{R}$, die die Diagramme

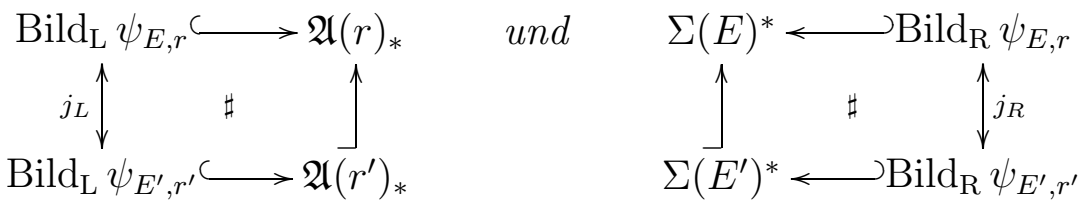

kommutativ machen. (Die Pfeile zwischen $\mathfrak{A}(r)_{*}$ und $\mathfrak{A}\left(r^{\prime}\right)_{*}$ sowie $\Sigma(E)^{*}$ und $\Sigma\left(E^{\prime}\right)^{*}$ sind je nach Größenverhältnis von E, $E^{\prime}, r, r^{\prime}$ ggf. umzukehren.)

Den Vektorraum aller Abbildungen aus $\bar{\Psi}$ von asymptotisch endlichem Rang bezeichnen wir $\operatorname{mit} \bar{\Psi}_{0}$.

In unserer Analyse der Punktfeldstruktur von Quantenfeldtheorien stützen wir uns auf solche Abbildungen von asymptotisch endlichem Rang. Sie besitzen für kleine Er ein „konstantes Bild“ und lassen sich, wie wir unten sehen werden, kanonisch in eine Summe von Rang-1-Abbildungen aus $\bar{\Psi}$ zerlegen.

Wir wollen jetzt untersuchen, wieweit sich die Existenz des Diagramms (2.C.4) für Abbildungen von asymptotisch endlichem Rang jetzt auch auf beliebige $E, E^{\prime}, r, r^{\prime}$ ausdehnen läßt. Von den vier zu behandelnden Fällen $E^{\prime} \leq E, r^{\prime} \leq r$ sind zwei sofort klar; wir behandeln nur $E^{\prime}>E, r^{\prime}<r$, der vierte Fall ergibt sich analog. Für kleine $E r, E^{\prime} r^{\prime}$ hat man per Definition

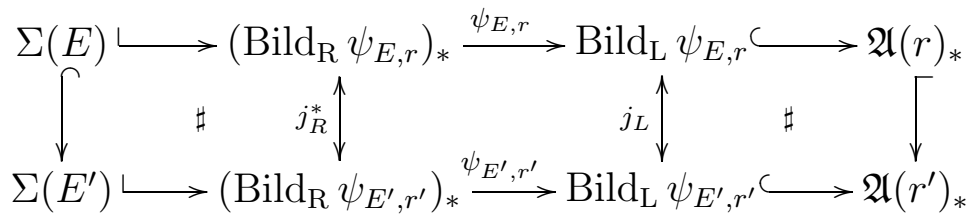

mit den geforderten Isomorphismen. Die beiden äußeren Vierecke kommutieren; fraglich ist lediglich, ob dies auch für das mittlere Viereck gilt.

Problematisch ist hier, daß wir wegen $E^{\prime}>E$, jedoch $r^{\prime}<r$ die Garbenverträglichkeit von $\psi$ nicht direkt ausnutzen können. Wir wählen uns daher $E^{\prime \prime} \geq E^{\prime}>E$ und $r^{\prime \prime} \geq r>r^{\prime}$ 
und behandeln dann das Diagramm:푼

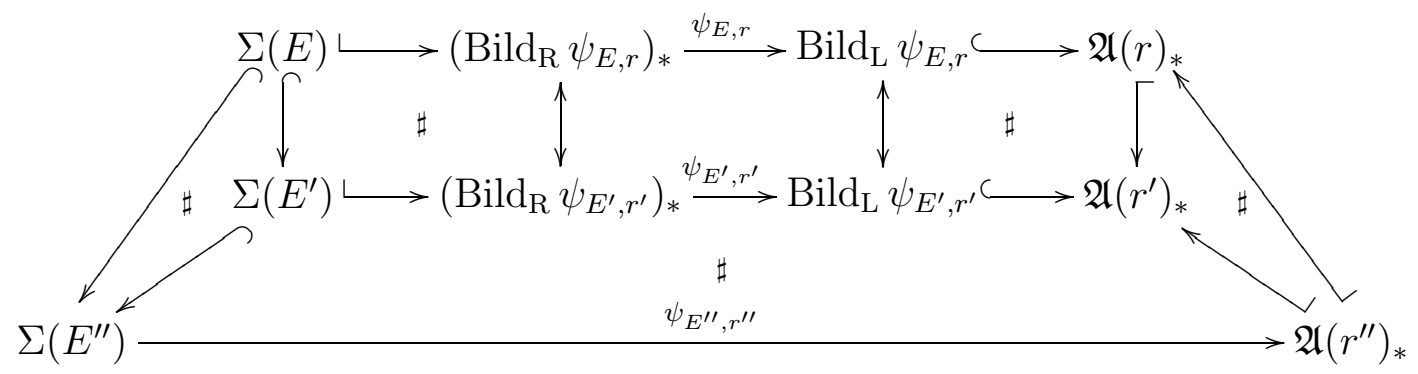

Wegen der Verträglichkeit von $\psi$ mit den Garbenstrukturen kommutiert hier

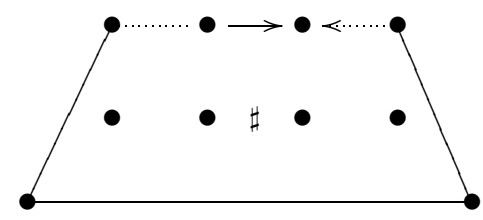

wobei mit gepunkteten Linien „zurückgezogene Abbildungen“ bezeichnet sind (Wahl eines Urbilds) - man muß sich dabei überzeugen, daß dies wohldefiniert ist (in diesem Fall ist das allerdings sehr einfach). Wegen der geforderten Kommutativität in wir (2.C.7) umformen zu

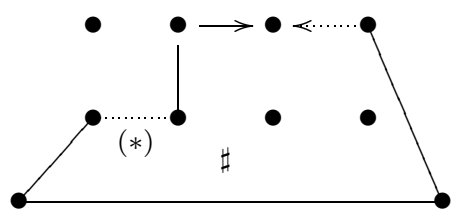

- hier wurde zusätzlich die Prägarben-Eigenschaft von $\bar{\Sigma}$ benutzt; bei der Zurückziehung $(*)$ ist ein Urbild zu wählen, das im Bild der Inklusion $\Sigma(E) \hookrightarrow \Sigma\left(E^{\prime}\right)$ liegt.

Weiter kann man die Urbildwahl $\bullet \bullet \bullet$ • (auf dem interessierenden Teilraum, wo Urbilder existieren) wegen der geforderten Kommutativität ersetzen durch $\mathbf{\bullet}: \mathbf{\imath} \mathfrak{\bullet}$; hier geht die Existenz der geforderten Isomorphismen ein. Zusammen mit der Garbeneigenschaft von $\overline{\mathfrak{A}}_{*}$ läßt sich damit (2.C.8) umrechnen in:

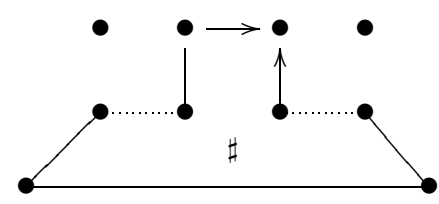

Verwendet man hier noch einmal die Garbenverträglichkeit von $\psi$, so erhält man die Kommutativität von

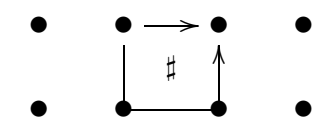

\footnotetext{
${ }^{12}$ Es sei bemerkt, daß wir für $E^{\prime \prime}, r^{\prime \prime}$ nicht fordern müssen, daß $\psi_{E^{\prime \prime}, r^{\prime \prime}}$ von endlichem Rang ist.
} 
wie gewünscht. Wir haben also gezeigt, daß in (2.C.5) auch das mittlere Quadrat kommutiert, was uns gewissermaßen ein „definiertes Verhalten“ von $\psi_{E, r}$ bei Änderung von $E$ und $r$ sicherstellt.

Wir bemerken insbesondere, daß $\operatorname{Bild}_{\mathrm{L}} \psi_{E, r}$, nicht von $E$ abhängt" in dem Sinne, daß bei festem $r$ und kleinen $E r, E^{\prime} r$ das Diagramm

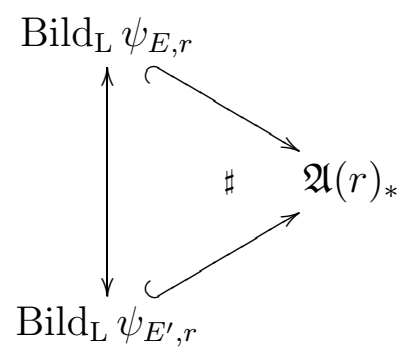

kommutiert; die Räume $\operatorname{Bild}_{\mathrm{L}} \psi_{E, r}$ und $\operatorname{Bild}_{\mathrm{L}} \psi_{E^{\prime}, r}$ sind also als Teilräume von $\mathfrak{A}(r)_{*}$ gleich. In derselben Weise ist Bild ${ }_{\mathrm{R}} \psi_{E, r}$ unabhängig von $r$.

Besonders interessiert auch hier wieder der Fall von (asymptotischen) Rang-1-Abbildungen. Eine solche Abbildung $\psi$ sei im folgenden gegeben. Wir wählen zunächst $E, r$ fest (Er genügend klein) und je ein Basiselement $\sigma_{r} \in \operatorname{Bild}_{\mathrm{L}} \psi_{E, r}$ und $\phi_{E} \in \operatorname{Bild}_{\mathrm{R}} \psi_{E, r}$, so daß $\psi_{E, r}=\phi_{E} \sigma_{r}$ (vgl. (2.B.17) ). Wir können $\psi_{E, r}$ also schreiben als

$$
\Sigma(E) \stackrel{\phi_{E}}{\longrightarrow}\left(\operatorname{Bild}_{\mathrm{R}} \psi_{E, r}\right)_{*} \cong \mathbb{C} \stackrel{\psi_{E, r}=1}{\longrightarrow} \mathbb{C} \cong \operatorname{Bild}_{\mathrm{L}} \psi_{E, r} \stackrel{\sigma_{r}}{\longrightarrow} \mathfrak{A}(r)_{*}
$$

wobei $\psi_{E, r}$ zwischen den beiden eindimensionalen Räumen einfach der Multiplikation mit dem Skalar 1 entspricht.

Nun definieren wir $\sigma_{r}^{\prime}$ für $r^{\prime}<r$ durch die für die Eigenschaft „von asymptotisch endlichem Rang“ geforderten Isomorphismen $j_{L}$ : Sie sollen $\sigma_{r}$ auf $\sigma_{r^{\prime}}$ transportieren, d.h. das Diagramm

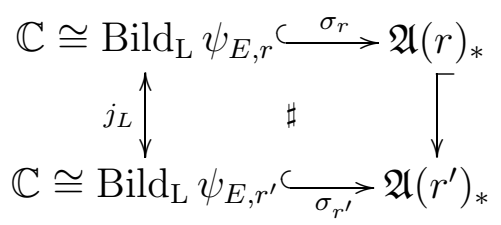

wird kommutativ gemacht. Für $r^{\prime}>r$ setzen wir $\sigma_{r}$ beliebig als Linearform auf $\mathfrak{A}\left(r^{\prime}\right)$ fort (das ist möglich, da $\mathfrak{A}(r)_{*}=\Sigma\lceil\mathfrak{A}(r)$ ). Damit wird $\sigma$ insgesamt zu einer wohldefinierten Linearform auf der Präkogarbe $\overline{\mathfrak{A}}$, also $\sigma \in \overline{\mathfrak{A}}_{*}$.

Ganz analog definieren wir $\phi_{E}$ auf anderen Räumen $\Sigma\left(E^{\prime}\right)$, indem wir die Isomorphismen $j_{R}$ verwenden bzw. verlangen, daß

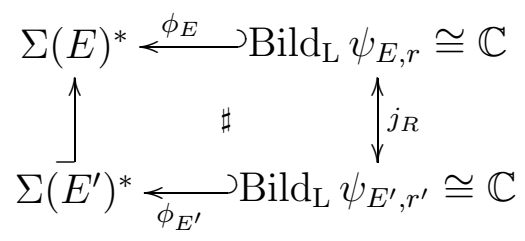

kommutativ wird. (Nach dem oben Gesagten können wir hier $r^{\prime}<r$ beliebig wählen, so daß wir sicherstellen können, daß $E^{\prime} r^{\prime}$ klein bleibt.) Damit ist also auch $\phi \in \bar{\Sigma}^{*}$ wohldefiniert. 
Diese Definition von $\sigma$ und $\phi$ ermöglicht es uns, für beliebige $E^{\prime}, r^{\prime}$ (jedoch kleine $E^{\prime} r^{\prime}$ ) die Abbildung $\psi_{E^{\prime}, r^{\prime}}$ folgendermaßen darzustellen:

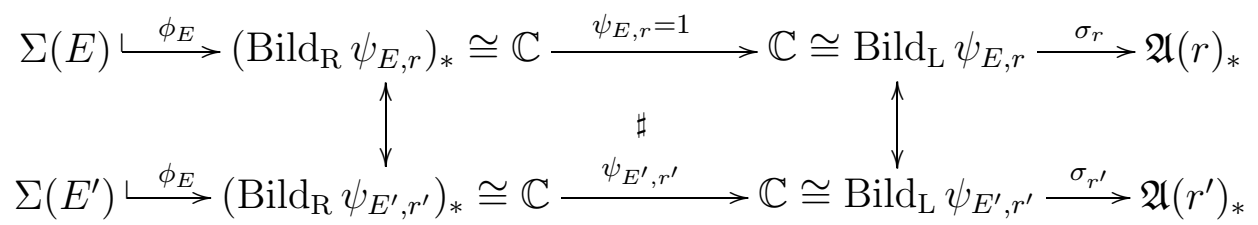

Dabei entspricht auch $\psi_{E^{\prime}, r^{\prime}}$ hier nur der Multiplikation mit einem Skalar; da wir nach (2.C.10) aber wissen, daß das mittlere Quadrat kommutiert, und da die Isomorphismen die gewählten Basiselemente aufeinander abbilden, ist auch dieser Skalar gleich 1. Wir erhalten auf diese Weise

$$
\psi \underset{\text { as }}{=} \phi \sigma
$$

mit $\sigma \in \overline{\mathfrak{A}}_{*}$ und $\phi \in \bar{\Sigma}^{*}$, wobei $\underset{\text { as }}{=}$ bedeutet, daß die Gleichung eventuell nur für kleine $E r$ gilt (asymptotische Gleichheit).

Abbildungen $\psi \in \bar{\Psi}_{0}$ mit asymptotischem Rang $n>1$ lassen sich entsprechend in Rang-1-Abbildungen zerlegen: Bei festem E,r zerlegen wir $\psi_{E, r}$ wie in Formel (2.B.23) und setzen diese Zerlegung dann mittels der Isomorphismen fort. Auf diese Weise erhalten wir:

Satz 2.3. Es sei $\psi \in \bar{\Psi}_{0}$ eine Abbildung vom asymptotischen Rang $n$. Dann gibt es linear unabhängige $\sigma_{j} \in \overline{\mathfrak{A}}_{*}$ und $\phi_{j} \in \bar{\Sigma}^{*}(j=1, \ldots, n)$, so daß

$$
\psi \underset{\text { as }}{=} \sum_{j=1}^{n} \phi_{j} \sigma_{j} .
$$

Da wir $\mathfrak{A}(r)_{*}=\Sigma\left\lceil\mathfrak{A}(r)\right.$ haben, können wir hier statt $\sigma_{j} \in \overline{\mathfrak{A}}_{*}$ auch $\sigma_{j} \in \Sigma$ annehmen und das Element der Prägarbe ggf. durch Restriktion zurückgewinnnen.

\section{D Asymptotisches Verhalten}

In diesem Abschnitt sei $F$ die Menge der Funktionen $\mathbb{R}^{+} \rightarrow \mathbb{R}^{+}$, die bei $x=0$ beschränkt sind. Wir werden für $f \in F$ in verschiedenem Zusammenhang das asymptotische Verhalten von $f(x)$ für $x \rightarrow 0$ analysieren und quantifizieren müssen; dazu sollen hier einige Begriffe eingeführt werden.

Asymptotische Ordnung Es seien $f, g \in F$ zwei Funktionen. Wir schreiben

$$
f \underset{\text { as }}{\leq} g \quad: \Leftrightarrow \quad f(x) \leq g(x) \cdot \text { const. für kleine } x(\text {,asymptotisch“ }
$$

Diese Relation liefert eine Prä-Ordnung auf der Menge $F$, d.h. wir erhalten eine Halbordnung modulo der Äquivalenzrelation

$$
f \underset{\text { as }}{\sim} g \quad: \Leftrightarrow \quad f \underset{\text { as }}{\sim} g \wedge g \underset{\text { as }}{\leq} f
$$


Weiter schreiben wir

$$
f \underset{\text { as }}{\ll} g \quad: \Leftrightarrow \quad \frac{f(x)}{g(x)} \underset{x \rightarrow 0}{\longrightarrow} 0 .
$$

Diese Begriffe lassen sich auch bezüglich einer festen Nullfolge $\rho$ definieren, d.h. in (2.D.1) und (2.D.3) wird nur $x \in \rho$ zugelassen; wir schreiben die entsprechenden Relationen als $\underset{\rho}{\leq}$, $\underset{\rho}{\sim}$ und $\underset{\rho}{\ll}$. Interessant ist das Verhalten beim Übergang zu Teilfolgen von $\rho$ : Gilt $f \underset{\rho}{\leq} g$ mit einer Nullfolge $\rho$, dann ist auch $f \leq \frac{1}{\rho^{\prime}}$ für jede Teilfolge $\rho^{\prime} \subset \rho$. Die Umkehrung ist aber im allgemeinen falsch; insbesondere könnte $f \underset{\rho^{\prime}}{\sim} g$ sein, aber $f \underset{\rho}{\sim} g$ (entsprechendes gilt für $\underset{\rho}{\ll}$ ). Durch Übergang zu Teilfolgen kann man die Ordnung also verfeinern: Gilt etwa $f \underset{\rho}{\ngtr} g$, dann gibt es offenbar eine Teilfolge $\rho^{\prime} \subset \rho$, so daß $f \underset{\rho^{\prime}}{\ll} g$. Wir können also nicht vergleichbare Elemente durch Übergang zu Teilfolgen vergleichbar machen.

Asymptotischer Exponent Einen quantitativeren Begriff des asymptotischen Verhaltens gibt folgende Definition: Für $f \in F$ setzen wir

$$
\gamma(f):=\sup \left\{\lambda \mid x^{-\lambda} f(x) \underset{x \rightarrow 0}{\longrightarrow} 0\right\}
$$

Wir nennen $\gamma(f)$ den asymptotischen Exponenten von $f$; er nimmt Werte im Bereich [0, $]$ an. (Wir lassen den Wert $\infty$ des Supremums hier zu.) Der Name „asymptotischer Exponent" ist gerechtfertigt, denn es gilt offenbar

$$
\gamma\left(x^{\lambda}\right)=\lambda \quad \forall \lambda \geq 0
$$

Weiter haben wir für beliebiges (aber festes) $\epsilon>0$, daß

$$
f \underset{\text { as }}{\ll} x^{\gamma(f)-\epsilon}, \quad f \underset{\rho}{\gg} x^{\gamma(f)+\epsilon} \text { mit einer geeigneten Nullfolge } \rho \text {. }
$$

Daraus ergibt sich sofort folgende Charakterisierung von $\gamma(\cdot)$ :

$$
\gamma(f) \geq \bar{\gamma} \quad \Leftrightarrow \quad x^{-\bar{\gamma}+\epsilon} f(x) \rightarrow 0 \forall \epsilon>0
$$

$\gamma(\cdot)$ respektiert die asymptotische Anordnung in dem Sinne, daß

$$
f \underset{\mathrm{as}}{\leq} g \quad \Rightarrow \quad \gamma(f) \geq \gamma(g)
$$

Bezüglich der Addition zweier Funktionen sieht man leicht, daß

$$
\gamma(f+g)=\min \{\gamma(f), \gamma(g)\}
$$

Eine weitere interessante Eigenschaft von $\gamma$ ist diese: Sei $\mu \in \mathbb{R}^{+}$fest, dann definieren wir zu $f \in F$ die skalierte Funktion $f_{\mu}(x):=f(\mu x)$. Für diese Funktion ist

$$
x^{-\lambda} f_{\mu}(x)=x^{-\lambda} f(\mu x) \underset{\left(x^{\prime}:=\mu x\right)}{=} \mu^{\lambda} x^{\prime-\lambda} f\left(x^{\prime}\right),
$$


was im Limes $x \rightarrow 0$ genau dann verschwindet, wenn $x^{-\lambda} f(x) \rightarrow 0$; es gilt also

$$
\gamma\left(f_{\mu}\right)=\gamma(f)
$$

d.h. der asymptotische Exponent ändert sich nicht bei Skalierung.

Um das potentielle Auftreten des Wertes $\gamma(\cdot)=\infty$ zu vermeiden, betrachten wir häufig auch

$$
\delta(f):= \begin{cases}\frac{1}{1+\gamma(f)} & \text { für } \gamma(f)<\infty \\ 0 & \text { für } \gamma(f)=\infty\end{cases}
$$

was dann im Intervall [0,1] liegt. Gleichung (2.D.9) liefert

$$
\delta(f+g)=\max \{\delta(f), \delta(g)\} \leq \delta(f)+\delta(g),
$$

also erfüllt $\delta(\cdot)$ die Dreiecksungleichung, und $(f, g) \mapsto \delta(f-g)$ ist eine Pseudometrik auf $F$.

Wir benötigen in der Anwendung noch einige Verallgemeinerungen dieses Schemas. Es sei dazu $V$ ein Vektorraum und $\varphi: V \rightarrow F$ eine Abbildung, die $\varphi(v)=\varphi(-v)$ erfülle und zusätzlich einer „,asymptotischen Dreiecksungleichung“ genüge:

$$
\varphi(u+v) \underset{\text { as }}{\leq} \varphi(u)+\varphi(v) \quad \text { für } u, v \in V .
$$

(Z.B. könnte $\varphi$ eine Halbnorm auf $V$ sein.) Wir definieren dann den asymptotischen Exponenten von $v \in V$ durch

$$
\gamma^{\prime}(v):=\gamma(\varphi(v))
$$

Als leicht abgeschwächtes Analogon von (2.D.9) haben wir dann

$$
\gamma^{\prime}(u+v)=\gamma(\varphi(u+v)) \underset{(2 . \bar{D} .8)}{\geq} \gamma(\varphi(u)+\varphi(v))=\min \left\{\gamma^{\prime}(u), \gamma^{\prime}(v)\right\} \text {. }
$$

Ist hier $\gamma^{\prime}(u) \neq \gamma^{\prime}(v)$ (wir können „,< annehmen), dann berechnet man analog

$$
\gamma^{\prime}(u)=\gamma^{\prime}((u+v)+(-v)) \geq \min \left\{\gamma^{\prime}(u+v), \gamma^{\prime}(v)\right\}
$$

also ist tatsächlich $\gamma^{\prime}(u+v)=\gamma^{\prime}(u)$, d.h. in (2.D.16) gilt Gleichheit.

Dementsprechend liefert $\delta^{\prime}:=\delta \circ \varphi$ eine Pseudometrik auf $V$.

Eine andere Verallgemeinerung ergibt sich wiederum dadurch, die Konvergenz auf eine bestimmte Nullfolge $\rho$ einzuschränken: Wir setzen

$$
\gamma_{\rho}(f):=\sup \left\{\lambda \mid x^{-\lambda} f(x) \underset{x \rightarrow 0}{\longrightarrow} 0\right\}
$$

$\gamma_{\rho}$ und das entsprechend definierte $\delta_{\rho}$ haben dann analoge Eigenschaften, wie sie zuvor für $\gamma$ und $\delta$ hergeleitet wurden. Auch eine Komposition mit einer Abbildung $\varphi$ des obigen Typs läßt sich analog bilden. 
Anwendung auf Zustände und bilineare Abbildungen Wir wollen die obigen Strukturen (insbesondere die Definition des asymptotischen Exponenten) nun auf die für unsere Analyse interessanten Objekte anwenden, nämlich auf die Elemente von $\overline{\mathfrak{A}}_{*}$ und $\bar{\Psi}$.

Das asymptotische Verhalten eines Funktionals $\sigma \in \overline{\mathfrak{A}}_{*}$ (respektive $\sigma \in \Sigma$ ) für $r \rightarrow 0$ können wir durch die Norm

$$
\|\sigma\|_{r}:=\| \sigma\lceil\mathfrak{A}(r) \|
$$

beschreiben. Sie liegt als Funktion von $r$ in unserer Funktionenklasse $F$, weshalb der asymptotische Exponent

$$
\gamma(\sigma):=\gamma\left(\|\sigma\|_{r}\right)
$$

wohldefiniert ist (man beachte, daß $\|\cdot\|_{r}$ die oben an die Abbildung $\varphi$ gestellten Voraussetzungen erfüllt).

Bilineare Abbildungen $\psi \in \bar{\Psi}$ wollen wir im Limes kleiner Wirkungen analysieren und betrachten dazu (wie zuvor in Abschnitt 2.3)

$$
\|\psi\|_{E, r}:=\sup _{\sigma \in \Sigma(E)_{1}} \sup _{A \in \mathfrak{A}(r)_{1}}\left|\psi_{E, r}(\sigma, A)\right|
$$

und

$$
f_{\psi}(w)=\sup _{E \cdot r \leq w} B\left(\|\psi\|_{E, r}\right) \quad \text { mit } \quad B(x)=\frac{x}{1+x}
$$

durch die „Kappung“ der großen Werte durch die Funktion $B$ liegt auch $f_{\psi}$ in $F$, und man überzeugt sich, daß $\varphi: \psi \mapsto f_{\psi}$ den obigen Voraussetzungen genügt. Damit können wir auch hier den asymptotischen Exponenten

$$
\gamma(\psi):=\gamma\left(f_{\psi}\right)
$$

betrachten.

(Das Einfügen der Funktion $B$ hat in den für uns relevanten Fällen auf den asymptotischen Exponenten keinen Einfluß: Für $f \in F$ gilt $\gamma(f)=\gamma(B \circ f)$, und falls $f$ eine positive Funktion mit $\gamma(B \circ f)>0$ ist, dann folgt $f \in F$.)

Der Vollständigkeit halber erwähnen wir noch die Definition

$$
\|\phi\|_{E}:=\| \phi\left\lceil\Sigma(E) \| \quad \text { für } \phi \in \bar{\Sigma}^{*} ;\right.
$$

damit wird für Rang-1-Abbildungen $\phi \sigma \in \bar{\Psi}_{0}$ offenbar

$$
\|\phi \sigma\|_{E, r}=\|\phi\|_{E}\|\sigma\|_{r} .
$$

Asymptotische Exponenten für $\|\phi\|_{E}$ werden wir nicht betrachten. 


\section{Kapitel 3}

\section{Konstruktion von Punktfeldern}

In diesem Kapitel behandeln wir die Bestimmung des Feldinhalts und die explizite Konstruktion von Wightman-Feldern im Rahmen der algebraischen Feldtheorie.

Wir gehen dazu aus von einer Theorie, die das im vorangegangenen Kapitel entwickelte asymptotische Phasenraumkriterium erfüllt. Anhand einer Analyse der approximierenden bilinearen Abbildungen im Limes kleiner Abstände zeichnen wir zunächst eine Hierarchie von endlichdimensionalen Vektorräumen aus, die anhand eines reellen Parameters geordnet sind. Diese Räume wollen wir als Feldinhalt etablieren; der reelle Parameter beschreibt die „asymptotische Dimension“ der Felder.

Unter der Zusatzvoraussetzung polynomialer Energieschranken können wir die Elemente dieser Räume (es handelt sich um quadratische Formen) durch Folgen beschränkter lokaler Operatoren approximieren. Die quadratischen Formen lassen sich dann zu WightmanFeldern ausintegrieren, wobei sich die Lokalitätseigenschaften der approximierenden Operatoren auf die Distributionen übertragen.

Ein Vergleich mit dem in der Literatur bekannten Fredenhagen-Hertel-Feldinhalt [FH81] zeigt nicht nur die Gleichheit mit den von uns berechneten Feldern, sondern wir erhalten außerdem explizitere Aussagen über die Kurzabstandsdivergenz bei Approximation der Felder durch beschränkte Operatoren.

Die konstruierten Felder sind affiliiert zum lokalen Netz und erfüllen die WightmanAxiome, wobei wir die Behandlung der Symmetrieaspekte auf Kapitel 1 verschieben. 


\subsection{Feldinhalt}

Wir hatten im letzten Kapitel ein Phasenraumkriterium definiert, das es uns ermöglichen sollte, Punktfelder im Rahmen der algebraischen Feldtheorie zu konstruieren. Wir betrachten nun eine Theorie, die dieses Kriterium erfüllt, und wollen ihren Feldinhalt bestimmen.

Das Kriterium besagt grob, daß die Auswertung lokaler Messungen (Abbildung $\Xi$ ) sich durch bilineare Abbildungen $\psi$ von endlichem Rang approximieren läßt; und die heuristische Motivation in Abschnitt 2.1 legt nahe, daß der Feldinhalt gerade aus dem Rechts-Bild dieser Abbildungen besteht. Dies ist aus verschiedenen Gründen im allgemeinen nicht der Fall, wie wir gleich sehen werden. Wir werden jedoch im Rechts-Bild gewisse Unterräume auszeichnen, die sich tatsächlich als der Feldinhalt der Theorie herausstellen.

Die Details stellen sich wie folgt dar: Das asymptotische Phasenraumkriterium „ $\delta_{n} \rightarrow 0$ “ (nach Definition 2.1) garantiert uns, daß zu jedem $\bar{\gamma}>0$ eine Abbildung $\psi \in \bar{\Psi}_{0}$ (respektive $\left.\bar{\Psi}_{0}^{P}\right)$ existiert, so daß

$$
\gamma(\Xi-\psi) \geq \bar{\gamma}
$$

Wir halten im folgenden $\bar{\gamma}$ und zunächst auch $\psi$ fest. Um die zu erwartende Situation zu klären, gehen wir zunächst zu einer Basisdarstellung von $\psi$ über und schreiben die Abbildung als eine (nicht eindeutig bestimmte) direkte Summe von Rang-1-Operatoren:

$$
\psi=\underset{\text { as }}{=} \sum_{j=1}^{\text {Rang } \psi} \phi_{j} \sigma_{j} \quad \text { mit } \phi_{j} \in \bar{\Sigma}^{*}, \sigma_{j} \in \Sigma \text {. }
$$

Unsere Vermutung ist, daß es sich bei den quadratischen Formen $\phi_{j}$ um lokale Punktfelder handelt. Dies kann aber im allgemeinen sicher nicht für alle der $\phi_{j}$ richtig sein. Gilt beispielsweise $\gamma\left(\sigma_{j}\right) \geq \bar{\gamma}$ für ein $j$, dann ist für beliebiges $B \in \mathfrak{B}(\mathcal{H})$

$$
\left\|B \sigma_{j}\right\|_{E, r} \leq\|B\|\left\|\sigma_{j}\right\|_{r} \quad \Rightarrow \quad \gamma\left(B \sigma_{j}\right) \geq \bar{\gamma}
$$

daher gilt auch

$$
\gamma\left(\Xi-\psi-B \sigma_{j}\right) \geq \bar{\gamma}
$$

und wir könnten in der Approximationssumme (3.1.2) folglich $\phi_{j}$ durch $B+\phi_{j}$ ersetzen, ohne die Eigenschaft (3.1.1) zu ändern. Sicher muß $B$ und damit $B+\phi_{j}$ aber im allgemeinen nicht am Koordinatenursprung lokalisiert sein.

Wir müssen solche Terme also ausschließen, wollen wir lokale Punktfelder im RechtsBild von $\psi$ identifizieren. Wir abstrahieren dazu von der Basisdarstellung (3.1.2) und definieren folgenden Unterraum von $S:=\operatorname{Bild}_{\mathrm{L}} \psi$ :

$$
S^{I I}:=\{\sigma \in S \mid \gamma(\sigma) \geq \bar{\gamma}\}
$$

Dann wählen wir einen weiteren Raum $S^{I} \subset S$, so daß

$$
S=S^{I} \oplus S^{I I}
$$

Wir nennen $S^{I}$ den primären, $S^{I I}$ den sekundären Anteil von $S$ (der Grund für diese Bezeichnung wird gleich noch klarer werden). Diese Aufspaltung ist hinsichtlich der Wahl 
von $S^{I}$ willkürlich und hängt außerdem vom gewählten Wert $\bar{\gamma}$ ab; dies unterdrücken wir allerdings in der Notation.

Die Zerlegung von $S$ in eine direkte Summe induziert (siehe Anhang 2.C) auch eine Zerlegung von $\operatorname{Bild}_{\mathrm{R}} \psi=: \Phi$ in zwei Räume

$$
\Phi=\Phi^{I} \oplus \Phi^{I I}
$$

so daß

$$
\psi=\psi^{I} \oplus \psi^{I I}
$$

bezüglich dieser Zerlegung in eine direkte Summe zerfällt. Dabei ist $\Phi^{I}$ als adjungierter Kern円 von $S^{I I}$ durch die Definition (3.1.5) eindeutig bestimmt, während $\Phi^{I I}$ von der Wahl von $S^{I}$ abhängt.

Die Zerlegung $\psi=\psi^{I} \oplus \psi^{I I}$ in primäre und sekundäre Anteile ist nun gerade die Aufspaltung, die oben am Beispiel der konkreten Basisdarstellung (3.1.2) erläutert wurde: Ein $\phi \in \Phi^{I I}=\operatorname{Bild}_{\mathrm{R}} \psi^{I I}$ korrespondiert gerade mit einem für $r \rightarrow 0$ schnell verschwindenden $\sigma \in S^{I I}$, weshalb man im allgemeinen nicht erwarten kann, daß $\phi$ ein lokales Punktfeld ist. Wir werden daher im folgenden nur den Raum $\Phi^{I}$ untersuchen („primäre Felder“). Für das Auftreten der „,sekundären“ Felder aus $\Phi^{I I}$, deren genauere Analyse in unserem Rahmen nicht möglich scheint, kann es folgende Ursachen geben:

1. Sie stammen aus „redundanten“ Terme, d.h. direkten Summanden $\hat{\psi}^{I I}$ von $\psi^{I I}$, die $\gamma\left(\hat{\psi}^{I I}\right) \geq \bar{\gamma}$ erfüllen. Konkret können dies Rang-1-Terme $\phi \sigma$ mit nicht zu singulärem Hochenergieverhalten, nämlich $\|\phi\|_{E} \leq E^{\bar{\gamma}} \cdot$ const., sein. Solche Terme könnte man stets zu $\psi$ hinzuaddieren, ohne die Approximationseigenschaft (3.1.1) zu ändern, bzw. man könnte ihr Auftreten vermeiden, indem man in (3.1.1) die Minimierung von $\operatorname{Rang} \psi$ fordert.

2. Es könnte außerdem der Fall auftreten, daß $\gamma\left(\psi^{I I}\right)<\bar{\gamma}$, so daß sich $\psi^{I I}$ nicht vollständig durch eine Minimierung von Rang $\psi$ eliminieren läßt. Konkret ist dabei an Rang-1-Terme $\phi \sigma$ zu denken, die etwa $\|\sigma\|_{r} \sim r^{\bar{\gamma}-\epsilon},\|\phi\|_{E} \sim E^{\bar{\gamma}+\epsilon}$ erfüllen $(\epsilon>0)$. Diese Terme, die man „energiedominiert“ nennen könnte, da sie in $E$ singulärer sind, als sie in $r$ abfallen, zählen zum Anteil $\psi^{I I}$, sind aber für die Approximation von $\Xi$ im Sinne der Gleichung (3.1.1) eventuell nicht verzichtbar.

Wir rechnen in physikalisch relevanten Modellen (explizit in der freien Feldtheorie, allgemeiner in allen asymptotisch dilatationsinvarianten Modellen) damit, daß keine sekundären Terme von Typ 2 auftreten - im allgemeinen Fall läßt sich ihr Vorhandensein aber wohl nicht ausschließen. Es sei jedoch bemerkt, daß sie in jedem Fall nicht zum Feldinhalt beitragen: Wir werden in Abschnitt 3.4 zeigen, daß die untersuchten primären Felder den Fredenhagen-Hertel-Feldinhalt der Theorie ausschöpfen, der in gewisser Weise „alle Punktfelder der Theorie“ enthält.

Wie angekündigt, werden wir uns für die folgende Analyse nur auf den Raum $\Phi^{I}$ beziehen. Wir hatten bereits gesehen, daß er unabhängig von der (willkürlichen) Wahl von $S^{I}$ ist; allerdings könnte er von der Wahl der approximierenden Abbildung $\psi$ abhängen,

\footnotetext{
${ }^{1}$ Zum Begriff des adjungierten Kerns siehe Anhang 2.B.
} 
die bisher festgehalten war. Tatsächlich ist $\Phi^{I}$ aber auch bei Variation von $\psi$ eindeutig bestimmt (bei festen $\bar{\gamma}$ ) und insofern eine intrinsische Eigenschaft der Theorie. Wir zeigen dies, indem wir folgende Charakterisierung von $\Phi^{I}$ herleiten:

Satz 3.1. Für $\sigma \in \Sigma(E)$, E beliebig, gilt:

$$
\sigma\left\lceil\Phi^{I}=0 \quad \Leftrightarrow \quad \gamma\left(\Xi_{\mathrm{L}} \sigma\right) \geq \bar{\gamma} .\right.
$$

Beweis. $\Phi^{I}$ ist bezüglich $\psi$ der adjungierte Kern von $S^{I I}$, und nach dessen Charakterisierung (siehe (2.B.22) ) gilt

$$
\psi_{\mathrm{L}} \sigma \in S^{I I} \Leftrightarrow \sigma\left\lceil\Phi^{I}=0 .\right.
$$

Nach Definition von $S^{I I}$ ist also

$$
\sigma\left\lceil\Phi^{I}=0 \quad \Leftrightarrow \quad \gamma\left(\psi_{\mathrm{L}} \sigma\right) \geq \bar{\gamma} .\right.
$$

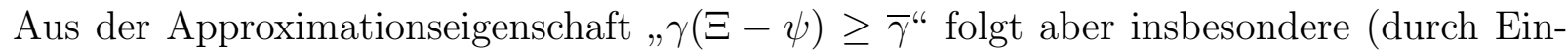
schränkung auf einen festen Wert von $E)$, daß

$$
\gamma\left(\psi_{\mathrm{L}} \sigma-\Xi_{\mathrm{L}} \sigma\right) \geq \bar{\gamma},
$$

und damit ergibt sich laut (2.D.16):

$$
\gamma\left(\psi_{\mathrm{L}} \sigma\right) \geq \bar{\gamma} \quad \Leftrightarrow \quad \gamma\left(\Xi_{\mathrm{L}} \sigma\right) \geq \bar{\gamma} .
$$

(3.1.10) und (3.1.12) liefern zusammen die Behauptung.

Die Eigenschaft,$\gamma\left(\Xi_{\mathrm{L}} \sigma\right) \geq \bar{\gamma}^{“}$ ist nun aber völlig unabhängig von der Wahl der approximierenden Abbildung $\psi$. Da der endlichdimensionale Raum $\Phi^{I}$ durch die Angabe der auf ihm verschwindenden Funktionale vollständig charakterisiert wird, ist er ebenfalls unabhängig von $\psi$ und hängt nur noch vom gewählten Wert $\bar{\gamma}$ ab.

Wir erhalten also (das asymptotische Phasenraumkriterium vorausgesetzt) zu jedem $\bar{\gamma}$ einen eindeutig festgelegten Raum $\Phi_{\bar{\gamma}}$ von „primären Feldern“. Der Vergleich dieser Räume bei verschiedenen $\bar{\gamma}$ ist sehr einfach. Ist $\bar{\gamma}^{\prime}>\bar{\gamma}$, dann erhalten wir nach dem Kriterium eine Abbildung $\psi \in \bar{\Psi}_{0}$ mit

$$
\gamma(\Xi-\psi) \geq \bar{\gamma}^{\prime}>\bar{\gamma}
$$

Da jetzt offenbar

$$
S^{I I\left(\bar{\gamma}^{\prime}\right)} \subset S^{I I(\bar{\gamma})}
$$

nach Definition dieser Räume (wobei $S^{I I(\bar{\gamma})}$ bezüglich der Zerlegung zu $\bar{\gamma}$ gebildet wird usw.), folgt sofort die umgekehrte Inklusion für die adjungierten Kerne:

$$
\Phi_{\bar{\gamma}}=\operatorname{Bild}_{\mathrm{R}} \psi^{I \bar{\gamma}} \subset \operatorname{Bild}_{\mathrm{R}} \psi^{I \bar{\gamma}^{\prime}}=\Phi_{\bar{\gamma}^{\prime}} .
$$

Damit haben wir folgenden Satz bewiesen: 
Satz 3.2. Die betrachtete Theorie erfülle das asymptotische Phasenraumkriterium. Dann gibt es zu jedem $\bar{\gamma}>0$ einen endlichdimensionalen Raum $\Phi_{\bar{\gamma}} \subset \bar{\Sigma}^{*}$ mit folgenden Eigenschaften:

- $\Phi_{\bar{\gamma}} \subset \Phi_{\bar{\gamma}^{\prime}}$ für $\bar{\gamma} \leq \bar{\gamma}^{\prime}$.

- Ist $\psi \in \bar{\Psi}_{0}$ eine Abbildung mit $\gamma(\Xi-\psi) \geq \bar{\gamma}$, dann ist $\Phi_{\bar{\gamma}}$ der adjungierte Kern des Raums

$$
S^{I I}:=\left\{\sigma \in \operatorname{Bild}_{\mathrm{L}} \psi \mid \gamma(\sigma) \geq \bar{\gamma}\right\}
$$

- Für $\sigma \in \bar{\Sigma}$ gilt

$$
\sigma\left\lceil\Phi_{\bar{\gamma}}=0 \quad \Leftrightarrow \quad \gamma\left(\Xi_{\mathrm{L}} \sigma\right) \geq \bar{\gamma}\right.
$$

Es sei bemerkt, daß durch das Phasenraumkriterium nicht garantiert wird, daß die Räume $\Phi_{\bar{\gamma}}$ nicht trivial sind: Auch falls $\delta_{n}>0 \forall n$, d.h. wenn man für die Approximation $\gamma(\Xi-\psi) \geq \bar{\gamma}$ immer einen gewissen minimalen Rang von $\psi$ benötigt, der mit $n$ ggf. sogar wächst, könnte trotzdem $\operatorname{dim} \Phi_{\bar{\gamma}}=0$ sein - nämlich dann, wenn $\operatorname{Bild}_{\mathrm{R}} \psi$ nur sekundäre Felder enthält. Bei gegebenem $\psi$ allerdings läßt sich die Dimension von $\Phi_{\bar{\gamma}}$ jedoch verhältnismäßig leicht bestimmen, indem man die Dimension von $S^{I I}$ ermittelt.

\subsection{Analyse des $r$-Verhaltens}

Unser Ziel ist es, die Elemente der Räume $\Phi_{\bar{\gamma}}$ als lokale Punktfelder zu etablieren. Dazu sei im folgenden wieder $\bar{\gamma}$ fest. Weiter sei $\psi \in \bar{\Psi}_{0}$ eine Abbildung mit $\gamma(\Xi-\psi) \geq \bar{\gamma}-$ ihre Existenz wird durch das asymptotische Phasenraumkriterium garantiert. Wir verwenden die Notation $S, S^{I}, S^{I I}, \Phi, \Phi^{I}, \Phi^{I I}$ wie zuvor; dabei ist $\Phi^{I}=\Phi_{\bar{\gamma}}$. Für $S^{I}$ bzw. $\Phi^{I I}$ bestehen noch gewisse Wahlfreiheiten, die wir auch ausnutzen werden.

Bei der Analyse von $\Phi^{I}$ tritt folgendes Problem auf: Wie wir noch explizit sehen werden, benötigt man sehr detaillierte Aussagen über das $r$-Verhalten der Funktionale $\sigma \in S^{I}$, um die quadratischen Formen $\phi \in \Phi^{I}$ durch beschränkte lokale Operatoren approximieren zu können. Bisher wissen wir nur, daß

$$
\sigma \in S^{I} \Rightarrow \gamma(\sigma)<\bar{\gamma}
$$

allerdings bedeutet dies im allgemeinen nicht, daß $\|\sigma\|_{r} \geq r^{\bar{\gamma}-\epsilon} \cdot$ const. für ein $\epsilon>0$; solch eine explizite Abschätzung läßt sich ggf. nur auf einer bestimmten Nullfolge $\rho$ etablieren, deren Auswahl aber von $\sigma$ abhängen kann. Dieser Umstand erschwert den technischen Teil der Analyse.

Wir werden aus diesem Grund auch noch andere Aufspaltungen von $S$ als das oben betrachtete $S^{I} \oplus S^{I I}$ betrachten. Es sei dazu $\rho \subset \mathbb{R}^{+}$eine Nullfolge. Wir definieren analog $\mathrm{zu}(3.1 .5)$ :

$$
S^{I I \rho}:=\left\{\sigma \in S \mid \gamma_{\rho}(\sigma) \geq \bar{\gamma}\right\}
$$


wobei

$$
\gamma_{\rho}(\sigma)=\sup \left\{\lambda \mid r^{-\lambda}\|\sigma\|_{r} \underset{\rho}{\rightarrow} 0\right\}
$$

und erhalten bei geeigneter Wahl eines Raums $S^{I \rho} \subset S$ eine Aufspaltung

$$
S=S^{I \rho} \oplus S^{I I \rho}, \quad \Phi=\Phi^{I \rho} \oplus \Phi^{I I \rho}, \quad \text { so daß } \psi=\psi^{I \rho} \oplus \psi^{I I \rho} .
$$

Wir sprechen hier von einer Zerlegung in $\rho$-primäre und $\rho$-sekundäre Anteile. Wiederum sind $S^{I I \rho}$ und sein adjungierter Kern $\Phi^{I \rho}$ durch die Definition (3.2.2) eindeutig bestimmt, während bei $S^{I \rho}$ bzw. $\Phi^{I I \rho}$ noch Wahlfreiheiten bestehen.

Aus der Definition ist sofort klar, daß

$$
S^{I \rho} \supset S^{I I} \quad \text { und damit } \quad \Phi^{I \rho} \subset \Phi^{I}
$$

ist weiterhin $\rho^{\prime} \subset \rho$ eine Teilfolge, dann gilt

$$
S^{I I \rho^{\prime}} \supset S^{I I \rho} \quad \text { und } \quad \Phi^{I \rho^{\prime}} \subset \Phi^{I \rho} \text {. }
$$

Im allgemeinen kann diese Inklusion echt sein; ist jedoch

$$
S^{I I \rho^{\prime}}=S^{I I \rho} \quad \text { und daher } \quad \Phi^{I \rho^{\prime}}=\Phi^{I \rho} .
$$

für alle Teilfolgen $\rho^{\prime} \subset \rho$, so nennen wir die Folge $\rho$ stabil. Offenbar kann man in jeder Nullfolge $\rho_{0}$ eine stabile Teilfolge $\rho$ finden, indem man unter allen Teilfolgen von $\rho_{0}$ eine auswählt, die die Dimension von $S^{I \rho}$ maximiert.

Die besondere Eigenschaft dieser stabilen Nullfolgen wird uns in die Lage versetzen, die Elemente von $\Phi^{I \rho}$ als lokale Punktfelder im Wightman-Sinne zu etablieren. (Dies benötigt allerdings noch einige Vorarbeiten.) Unsere Strategie ist also zunächst, $\Phi^{I}$ durch solche Räume $\Phi^{I \rho}$ mit unterschiedlichen $\rho$ zu überdecken oder zumindest aufzuspannen.

Eine gewisse Schwierigkeit dabei ist, daß die Folge $\rho$ offenbar nicht abhängig von einem festen $\phi \in \Phi^{I}$, sondern vielmehr zu einem gegebenem $\sigma \in S^{I}$ gewählt werden muß - die Auswahl der Folge soll ja zur Analyse des $r$-Verhaltens dienen. Dieser Umstand erfordert einige Umwege bei der Konstruktion der gesuchten Überdeckung von $\Phi^{I}$. Der folgende, etwas technisch wirkende Satz bildet hierfür die Grundlage.

Satz 3.3. Sei $\hat{\Phi} \subsetneq \Phi$ ein echter Teilraum von $\Phi$, so daß $\Phi^{I} \not \subset \hat{\Phi}$. Dann gibt es eine stabile Nullfolge $\rho$, so daß $\Phi^{I \rho} \not \subset \hat{\Phi}$.

Beweis. Wir dürfen ohne Einschränkung annehmen, daß $\hat{\Phi}$ in $\Phi$ die Kodimension 1 besitzt. (Andernfalls ergänzen wir $\hat{\Phi}$ vorher durch geeignete Basiselemente aus $\Phi$, ohne die Bedingung $\Phi^{I} \not \subset \hat{\Phi}$ zu verändern.) Dann gilt mit einem im folgenden festen $\phi_{0} \in \Phi^{I}$, $\phi_{0} \notin \hat{\Phi}$ :

$$
\Phi=\mathbb{C} \phi_{0} \oplus \hat{\Phi}
$$

Das induziert eine Zerlegung

$$
S=\mathbb{C} \sigma_{0} \oplus \hat{S}
$$


mit einem Raum $\hat{S} \subset S$ der Kodimension 1 und einem $\sigma_{0} \in S$, das durch $\hat{\Phi}$ eindeutig bestimmt wird. Da $\phi_{0} \in \Phi^{I}$, ist $S^{I I}$ Teil des adjungierten Kerns von $\mathbb{C} \phi_{0}$, also $S^{I I} \subset \hat{S}$; es gilt also $\sigma_{0} \notin S^{I I}$ und damit $\gamma\left(\sigma_{0}\right)<\bar{\gamma}$. Wir wählen nun eine Nullfolge $\rho$, so daß $\left\|\sigma_{0}\right\|_{r} \geq$ $r^{\bar{\gamma}-\epsilon}$ für ein $\epsilon>0$. Durch eventuellen Übergang zu einer Teilfolge können wir annehmen, daß $\rho$ stabil ist (die Abschätzung an $\left\|\sigma_{0}\right\|_{r}$ bleibt dabei erhalten). Es gilt insbesondere

$$
\sigma_{0} \notin S^{I I \rho} \text {. }
$$

Wir können dann $S^{I}$ und $S^{I \rho}$ so wählen (hier bestand ja noch Wahlfreiheit), daß

$$
\mathbb{C} \sigma_{0} \subset S^{I \rho} \subset S^{I}
$$

Weiter können wir $S^{I I \rho}$ so zu einem Raum $\check{S}$ ergänzen, daß

$$
S=\mathbb{C} \sigma_{0} \oplus \check{S}, \quad \check{S} \supset S^{I I \rho} .
$$

Wir haben damit folgendes Bild:

$$
\begin{aligned}
& S=\quad S^{I} \oplus S^{I I} \\
& \cup \quad \cap \\
& S=\quad S^{I \rho} \oplus S^{I I \rho} \\
& S=\quad \mathbb{C} \sigma_{0} \oplus \quad \check{S}
\end{aligned}
$$

Übertragen wir dies durch Übergang zu den adjungierten Kernen in den Raum $\Phi$, so erhalten wir:

$$
\begin{aligned}
& \Phi=\quad \Phi^{I} \quad \oplus \quad \Phi^{I I} \\
& \cup \quad \cap \\
& \Phi=\quad \Phi^{I \rho} \quad \oplus \quad \Phi^{I I \rho} \\
& \Phi=\quad \mathbb{C} \phi_{0}^{\prime} \oplus \quad \hat{\Phi}
\end{aligned}
$$

Hierbei ist $\phi_{0}^{\prime}$ als Basisvektor des eindimensionalen adjungierten Kerns von $\check{S}$ gegeben. Wir haben damit ein $\phi_{0}^{\prime} \in \Phi^{I \rho}, \phi_{0}^{\prime} \notin \hat{\Phi}$ gefunden; also ist $\Phi^{I \rho} \not \subset \hat{\Phi}$.

Dieser Satz läßt sich nun folgendermaßen anwenden: Wir konstruieren eine Basis von $\Phi^{I}$ induktiv aus Elementen von Räumen $\Phi^{I \rho}$ zu verschiedenen Nullfolgen $\rho$. Wir beginnen mit dem nulldimensionalen Raum $\hat{\Phi}_{0}:=\{0\} \subset \Phi^{I}$. Ist $\hat{\Phi}_{j} \subsetneq \Phi^{I}$, dann finden wir nach dem obigen Satz eine stabile Nullfolge $\rho_{j}$ und ein $\phi_{j} \in \Phi^{I \rho_{j}}, \phi_{j} \notin \hat{\Phi}_{j}$. Der Raum $\hat{\Phi}_{j+1}:=$ $\operatorname{Span}\left\{\phi_{j}, \hat{\Phi}_{j}\right\}$ ist also größer als $\hat{\Phi}_{j}$ (explizit ist $\operatorname{dim} \hat{\Phi}_{j}=j$ ). Nach endlich vielen Schritten muß das Verfahren abbrechen $\left(\hat{\Phi}_{J}=\Phi^{I}\right.$ mit einem gewissen $\left.J\right)$. Da $\hat{\Phi}_{J}$ per Konstruktion eine Basis aus primären Feldern bezüglich gewisser stabiler Nullfolgen besitzt, haben wir also gezeigt: 
Satz 3.4. Sei $\psi \in \bar{\Psi}_{0}$, so daß $\gamma(\Xi-\psi) \geq \bar{\gamma}$. Dann gilt

$$
\Phi^{I}=\operatorname{Span}\left(\bigcup_{\rho} \Phi^{I \rho}\right)
$$

wobei die Vereinigung über alle bezüglich $\psi$ und $\bar{\gamma}$ stabilen Nullfolgen $\rho$ läuft.

Unser Fernziel war es, die Elemente von $\Phi^{I}$ als lokale Punktfelder zu identifizieren; da dies ein „linearer Begriff“" ist, können wir uns dabei also auf die Räume $\Phi^{I \rho}$ konzentrieren.

Im folgenden sei die Folge $\rho$ fest gegeben. Wir betrachten die Aufspaltung von $\psi$ in $\rho$-primäre und $\rho$-sekundäre Anteile:

$$
\psi=\psi^{I \rho} \oplus \psi^{I I \rho} .
$$

Wir interessieren uns dabei nur für die Anteile $\psi^{I \rho}$; wie aber oben schon bemerkt, kann auch der Anteil $\psi^{I I \rho}$ für die Approximation $\gamma(\Xi-\psi) \geq \bar{\gamma}$ wesentlich sein. Wir können diesen Anteil allerdings dann vernachlässigen, wenn wir im Limes die Dämpfung der hochenergetischen Anteile erhöhen, und zwar in folgendem Sinne: Wir schreiben

$$
\psi^{I I \rho}=\sum_{j} \phi_{j} \sigma_{j} \quad \text { mit } \sigma_{j} \in S^{I I \rho} \text { und } \phi_{j} \in \Phi^{I I \rho}
$$

Dann gilt offenbar

$$
\lim _{r \rightarrow 0} r^{-\bar{\gamma}+\epsilon}\left\|\sigma_{j}\right\|_{r}=0 \quad \forall \epsilon>0 .
$$

Setzen wir

$$
g(E)=\max \left\{\left\|\phi_{j}\right\|_{E}, E^{\bar{\gamma}}\right\}
$$

dann ist trivialerweise

$$
\frac{\left\|\phi_{j}\right\|_{E}}{g(E)} \leq \text { const. für alle } E \text { und } j
$$

und deshalb wird?

$$
\limsup _{E r \rightarrow 0} \frac{\left\|\psi^{I I \rho}\right\|_{E, r}}{g(E) r^{\bar{\gamma}-\epsilon}} \leq \sum_{j} \limsup _{E r \rightarrow 0} \frac{\left\|\phi_{j}\right\|_{E}\left\|\sigma_{j}\right\|_{r}}{g(E) r^{\bar{\gamma}-\epsilon}}=0 .
$$

${ }^{2}$ Hier sollte

$$
\limsup _{E r \rightarrow 0} f(E, r)
$$

genauer geschrieben werden als

$$
\lim _{w \rightarrow 0} \sup \{f(E, r) \mid E \in[1, \infty) ; r \in \rho ; E r \leq w\}
$$


Andererseits haben wir

$$
\gamma(\Xi-\psi) \geq \bar{\gamma}>0 \stackrel{(\text { 2.D.7) }}{\Rightarrow} \limsup _{E r \rightarrow \rho} \frac{\|\Xi-\psi\|_{E, r}}{g(E) r \bar{\gamma}-\epsilon}=0 .
$$

Die Dreiecksungleichung liefert aus (3.2.20) und (3.2.21) sofort

$$
\limsup _{E r \rightarrow 0} \frac{\left\|\Xi-\psi^{I \rho}\right\|_{E, r}}{g(E) r^{\bar{\gamma}-\epsilon}}=0 \quad \forall \epsilon>0 .
$$

In diesem Sinne der Approximation von $\Xi$ sind die $\rho$-sekundären Anteile von $\psi$ also redundant; die „energiedominierten Terme“ wurden quasi durch den Faktor $g(E)$, weggedämpft“. Bemerkt sei, daß im Fall $\psi \in \bar{\Psi}_{0}^{P}$ offenbar $g(E)$ polynomial beschränkt ist - siehe dazu (3.2.18) -, d.h. wir können $g(E)=E^{l}$ mit einem gewissen $l>0$ annehmen.

Der nächste zu klärende Punkt ist die genaue Struktur des Raums $S^{I \rho}$. Bisher wissen wir nur, daß für $\sigma \in S^{I \rho}$ stets gilt

$$
\gamma_{\rho}(\sigma)<\bar{\gamma}
$$

was aber für unsere Zwecke nicht ausreicht. Zur Analyse können wir Satz 3.23 aus Anhang 3.A verwenden: Danach gibt es eine Teilfolge $\rho^{\prime} \subset \rho$ und eine Zerlegung

$$
S^{I \rho}=S_{1} \oplus \ldots \oplus S_{J}
$$

mit gewissem $J \in \mathbb{N}$, wobei die Räume $S_{j}$ bezüglich der Teilfolge $\rho^{\prime}$ homogen sind, d.h. es gibt Funktionen $\eta_{j}$, so daß

$$
\|\sigma\|_{r} \underset{\rho^{\prime}}{\sim} \eta_{j} \quad \forall \sigma \in S_{j}, \quad \text { was wir auch notieren als: } S_{j} \underset{\rho^{\prime}}{\sim} \eta_{j} .
$$

Zusätzlich gilt

$$
\eta_{1} \underset{\rho^{\prime}}{\gg} \eta_{2} \underset{\rho^{\prime}}{\gg} \ldots \underset{\rho^{\prime}}{\gg} \eta_{J}
$$

In diesem Sinne haben wir jetzt das Links-Bild von $\psi^{I \rho}$ vollständig nach dem $r$-Verhalten der enthaltenen Funktionale geordnet. Da $\rho$ eine stabile Nullfolge ist, wissen wir wegen (3.2.23) und (3.2.25), daß

$$
\gamma_{\rho^{\prime}}\left(\eta_{j}\right)<\bar{\gamma} \quad \forall j
$$

Durch weiteren Übergang zu einer Teilfolge $\rho^{\prime \prime} \subset \rho^{\prime}$ können wir dann erreichen (Anhang 2.D), daß

$$
\eta_{j} \underset{\rho^{\prime \prime}}{\gg} r^{\bar{\gamma}-\delta} \quad \text { mit gewissem } \delta>0
$$

Alle anderen oben erhaltenen Eigenschaften bleiben auch bezüglich $\rho^{\prime \prime}$ erhalten. Wir formalisieren diese in einem eigenen Begriff:

Definition 3.5. Ein Tripel $\left(\rho, g,\left\{\psi_{j}\right\}\right)$, bestehend aus 
- einer Nullfolge $\rho$,

- einer monoton wachsenden Funktion $g(E):[1, \infty) \rightarrow \mathbb{R}^{+}$,

- einer endlichen Menge $\left\{\psi_{j}\right\} \subset \bar{\Psi}_{0}$ von bilinearen Abbildungen von asymptotisch endlichem Rang (wir numerieren $j=1 \ldots J$ )

heiße homogene Approximation zur Genauigkeit $\bar{\gamma}>0$, wenn

- $\psi_{1}+\ldots+\psi_{J}=\psi_{1} \oplus \ldots \oplus \psi_{J} \quad$ direkte Summe ist,

- die Räume $\operatorname{Bild}_{\mathrm{L}} \psi_{j}$ homogen sind, und mit $\operatorname{Bild}_{\mathrm{L}} \psi_{j} \underset{\rho}{\sim} \eta_{j}$ gilt $\eta_{1} \gg \ldots \gg_{\rho} \eta_{J} \gg r^{\bar{\gamma}-\delta}$ mit einem $\delta>0$

$$
\limsup _{E r \rightarrow 0} \frac{\left\|\Xi-\oplus \psi_{j}\right\|_{E, r}}{g(E) r^{\bar{\gamma}-\epsilon}}=0 \quad \forall \epsilon>0 .
$$

Im Falle $\left\{\psi_{j}\right\} \subset \bar{\Psi}_{0}^{P}$ und $g(E)=E^{l}$ mit gewissem $l>0$ sprechen wir von einer homogenen Approximation mit polynomialen Energieschranken.

Wir werden im Zusammenhang mit homogenen Approximationen die Elemente der endlichen Menge $\left\{\psi_{j}\right\}$ meist stillschweigend mit $j=1 \ldots J$ durchnumerieren und ebenso die Notation $\operatorname{Bild}_{\mathrm{L}} \psi_{j} \underset{\rho}{\sim} \eta_{j}$ verwenden. Es sei aber betont, daß $J$ und die Funktionen $\eta_{j}$ keine eigentlich neuen Daten sind, sondern durch die Angabe der $\phi_{j}$ schon festgelegt werden (bis auf Äquivalenz $\underset{\rho}{\sim}$ ).

Unsere oben erhaltene Aussage läßt sich jetzt so formulieren:

Satz 3.6. Es sei $\bar{\gamma}>0$ fest, $\psi \in \bar{\Psi}_{0}$ eine Abbildung mit $\gamma(\Xi-\psi) \geq \bar{\gamma}$ und $\rho$ eine bezüglich $\bar{\gamma}$ und $\psi$ stabile Nullfolge. Dann gibt es eine homogene Approximation

$$
\left(\rho^{\prime}, g,\left\{\psi_{j}\right\}\right)
$$

wobei $\rho^{\prime} \subset \rho$ eine Teilfolge ist und

$$
\Phi^{I \rho}=\bigoplus_{j} \operatorname{Bild}_{\mathrm{R}} \psi_{j}
$$

Zusammengefaßt mit Satz 3.4 erhält man:

Korollar 3.7. Die betrachtete Theorie erfülle das asymptotische Phasenraumkriterium. Dann gibt es bei festem $\bar{\gamma}>0$ homogene Approximationen $\left(\rho_{i}, g_{i},\left\{\psi_{j, i}\right\}\right)$ zur Genauigkeit $\bar{\gamma}$ (i durchläuft eine endliche Indexmenge $I$ ), so daß

$$
\Phi_{\bar{\gamma}}=\operatorname{Span}\left(\bigcup_{i \in I} \bigoplus_{j=1}^{J_{i}} \operatorname{Bild}_{\mathrm{R}} \psi_{j, i}\right) .
$$

Ist das asymptotische Phasenraumkriterium mit polynomialen Energieschranken erfüllt, dann können hier auch homogene Approximationen mit polynomialen Energieschranken gewählt werden.

Wir haben im folgenden also lediglich nachzuweisen, daß die Rechts-Bilder solcher homogenen Approximationen aus lokalen Punktfeldern bestehen. 


\subsection{Felder als Grenzwerte lokaler Operatoren}

Nachdem uns die Analyse der Approximationsabbildungen auf den Begriff der homogenen Approximationen geführt hat, gehen wir nun daran, die Elemente ihres Rechts-Bildes als Grenzwerte lokaler Observablen darzustellen. Wir konstruieren Folgen von beschränkten Operatoren, die in immer kleineren Gebieten lokalisiert sind, und die unter einer geeigneten Energiedämpfung gegen die in Rede stehenden quadratischen Formen konvergieren. Dies wird uns später ermöglichen, die Lokalitätseigenschaften der approximierenden Observablen auf die Grenzwerte zu übertragen und diese dann als lokale Wightman-Felder zu interpretieren.

Dieser Grenzprozeß läßt sich allerdings in der gewünschten Weise nur dann ausführen, wenn zusätzliche Einschränkungen an das Hochenergieverhalten der Abbildungen $\psi \in \bar{\Psi}_{0}$ gestellt werden. (Dies wird unten noch genauer diskutiert.) Wir werden dabei stets nur polynomiale Hochenergieschranken behandeln.

Motivation und Ansatzpunkt Wir betrachten eine homogene Approximation $\Pi=$ $\left(\rho, g,\left\{\psi_{j}\right\}\right)$ im Sinne von Definition 3.5. Für ein gegebenes $\phi \in \Phi_{j}:=\operatorname{Bild}_{\mathrm{R}} \psi_{j}$ suchen wir eine Folge von lokalen Operatoren, die gegen $\phi$ konvergiert. Genauer soll zunächst $P(E) \phi P(E) \in \Sigma(E)^{*}$ durch $P(E) A_{r} P(E)$ mit lokalen $A_{r} \in \mathfrak{A}(r)$ approximiert werden. Wir können den letzteren Ausdruck so schreiben:

$$
P(E) A_{r} P(E)=\Xi_{E, r}\left(\cdot, A_{r}\right)=\Xi_{\mathrm{R} E, r} A_{r} .
$$

Die Approximationseigenschaft von $\Pi$ bedeutet grob, daß

$$
\Xi_{\mathrm{R} E, r} A_{r} \approx\left(\oplus \psi_{j}\right)_{\mathrm{R} E, r} A_{r} \quad \text { im Limes } r \rightarrow 0 ;
$$

es liegt daher nahe, $A_{r}$ als ein Urbild von $\phi$ unter $\left(\bigoplus \psi_{j}\right)_{\mathrm{R} E, r}$ zu wählen, so daß dann

$$
P(E) \phi P(E)=\left(\oplus \psi_{j}\right)_{\mathrm{R} E, r} A_{r} \approx \Xi_{\mathrm{R} E, r} A_{r}=P(E) A_{r} P(E)
$$

gilt. Wir werden von dieser Idee etwas abweichen und zunächst nur einen einzelnen Summanden $\psi_{j}$ mit homogenem Links-Bild betrachten - die notwendige Erweiterung wird später diskutiert.

Es sei also allgemein $\hat{\psi} \in \bar{\Psi}_{0}$ mit homogenem Links-Bild gegeben. Unser Ziel ist es, zu einem gegebenen $\phi \in \operatorname{Bild}_{\mathrm{R}} \hat{\psi}$ ein Urbild unter $\hat{\psi}_{\mathrm{R}}$ in $\mathfrak{A}(r)$ zu finden, dessen Norm wir kontrollieren können. Wir erhalten bei festem $E$ und $r$ folgendes Diagramm:

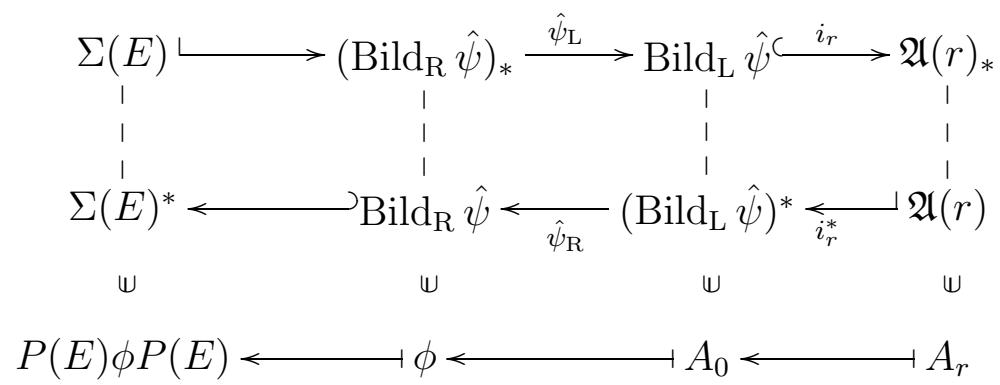

Wir können dabei zu $\phi$ sicher ein (eindeutiges) Urbild $A_{0} \in\left(\operatorname{Bild}_{\mathrm{L}} \hat{\psi}\right)^{*}$ wählen, da $\hat{\psi}_{\mathrm{R}}$ im obigen Diagramm ein Isomorphismus ist. Diese Wahl ist „unabhängig von $r$ ". Zu klären ist, 
welches Urbild $A_{r}$ man unter der jeweiligen Projektion $\mathfrak{A}(r) \longleftrightarrow\left(\operatorname{Bild}_{\mathrm{L}} \hat{\psi}\right)^{*}$ zu wählen hat, wenn man dabei seine Norm als Funktion von $r$ abschätzen möchte. Außerdem soll ein solches Urbild linear in $\phi$ gewählt werden, d.h. wir suchen eine Rechtsinverse zur Projektion $i_{r}^{*}$. Der folgende Satz garantiert ihre Existenz:

Satz 3.8. Sei $\hat{\psi} \in \bar{\Psi}_{0}$ eine Abbildung mit homogenem Links-Bild bezüglich einer Nullfolge $\rho$ :

$$
\operatorname{Bild}_{\mathrm{L}} \hat{\psi}=: S \underset{\rho}{\sim} \eta
$$

Dann gibt es ein $c>0$ und zu jedem $r \in \rho$ einen linearen Operator $F_{r}: S^{*} \rightarrow \mathfrak{A}(r)$ mit

$$
i_{r}^{*} \circ F_{r}=\operatorname{id}_{S^{*}} \quad \text { und } \quad\left\|F_{r}\right\| \leq \frac{c}{\eta(r)}
$$

Beweis. Wir definieren zunächst einen Operator $F_{r *}$ vom Teilraum $S \subset \mathfrak{A}(r)_{*}$ (genauer $\left.i_{r} S \subset \mathfrak{A}(r)_{*}\right)$ nach $S$ als Umkehrung der Inklusion $i_{r}$. Für $\sigma \in i_{r} S$ gilt dann

$$
\left\|F_{r *} \sigma\right\|_{S}=\|\sigma\|_{S} \leq \frac{c}{\eta(r)}\|\sigma\|_{r}
$$

mit einem festen $c>0$, da $S$ homogen ist (siehe Satz 3.21 in Anhang 3.A). Abstrakter ist also

$$
\| F_{r *}\left\lceil i_{r} S \| \leq \frac{c}{\eta(r)}\right.
$$

Wir setzen diesen Operator $F_{r *}$ nun mit Hilfe des Satzes von Hahn-Banach fort (bzw. mit einer leichten Erweiterung davon, siehe Satz 3.24 in Anhang 3.B) zu einem Operator $F_{r *}$ auf ganz $\mathfrak{A}(r)_{*}$ mit gleicher Normschranke (bis auf eine multiplikative Konstante). Für diesen fortgesetzten Operator gilt per Konstruktion

$$
F_{r *} \circ i_{r}=\mathrm{id}_{S}
$$

Setzen wir nun $F_{r}=\left(F_{r *}\right)^{*}$, dann haben wir einen Operator mit den im Satz behaupteten Eigenschaften gefunden, denn $\left\|F_{r}\right\|=\left\|\left(F_{r *}\right)^{*}\right\|=\left\|F_{r *}\right\|$.

Für den Fall einer homogenen Approximation mit nur einem Summanden $(J=1)$ haben wir also das gewünschte Urbild gefunden, und man wird nach (3.3.3) vermuten, daß $A_{r}:=F_{r} \circ \hat{\psi}_{R}^{-1} \phi$ für $r \rightarrow 0$ gegen $\phi$ konvergiert. Die genaue Formulierung dieser Konvergenz sowie die Erweiterung auf $J \neq 1$ stellen wir zunächst zurück, da vorher noch die verwendete Energiedämpfung genauer betrachtet werden muß.

\footnotetext{
${ }^{3}$ Dabei bezeichnet $\|\cdot\|_{S}$ eine Norm auf $S$. Zwischen dem Funktional $\sigma \in i_{r} S$ und seinem Urbild in $S$ unterscheiden wir in der Notation nicht.
} 
Aufweichung der Energieschranken Wir haben bisher die quadratischen Formen $\phi$ stets mit einer scharfen Energieschranke $P(E)$ betrachtet. Es scheint in diesem Zusammenhang natürlich, die Konvergenz einer Folge $A_{r} \in \mathfrak{A}(r)$ gegen eine quadratische Form $\phi$ „unter Energiedämpfung“" zu formulieren als

$$
P(E) A_{r} P(E) \rightarrow P(E) \phi P(E) \quad \forall E>0
$$

also i.W. als Konvergenz im Sinne von Linearformen auf $\bar{\Sigma}$. Eine solche Aussage erweist sich aber nicht als ausreichend, um später die Lokalität der Punktfelder zu gewährleisten. Wir gehen daher zu den schon von Fredenhagen und Hertel [FH81] verwendeten polynomialen Energieschranken über: Wir setzen

$$
R:=\frac{1}{1+H}
$$

und suchen dann nach einer Konvergenz der Art

$$
R^{l} A_{r} R^{l} \rightarrow R^{l} \phi R^{l} \quad \text { für ein } l>0 \text {. }
$$

Offenbar setzt dies Restriktionen an das Hochenergieverhalten von $\phi$ voraus, und wir werden deshalb im folgenden nur noch quadratische Formen bzw. homogene Approximationen mit polynomialen Energieschranken betrachten. Für die physikalische Anwendung sollte dies keine große Einschränkung darstellen, wie in Abschnitt 2.3 bereits diskutiert. Zwar sind die Ideen aus [FH81] von verschiedenen Autoren auch auf Felder mit anderem, z.B. exponentiellem Hochenergieverhalten verallgemeinert worden [RW86, Wol85, Sum87]; jedoch werden wir diese Überlegungen hier nicht aufgreifen, zumal auch das Phasenraumkriterium auf die Beschreibung von Theorien mit polynomialem Kurzabstandsverhalten ausgelegt ist - wir approximieren die Abbildung $\Xi$ stets mit einer „polynomialen Genauigkeit" $(E r)^{\bar{\gamma}}$.

Wir diskutieren nun den formalen Übergang von der bisher verwendeten scharfen Energiedämpfung zu derjenigen mit Faktoren $R^{l}$ (nach Fredenhagen und Hertel). Zunächst ist die Beschränktheit von $R^{l} \phi R^{l}$ äquivalent mit dem bisher diskutierten polynomialen Hochenergieverhalten:

Lemma 3.9. Für festes $\phi \in \bar{\Sigma}^{*}$ sind folgende Aussagen äquivalent:

1. Es gibt ein $k>0$, so daß $\|\phi\|_{E} \leq E^{k}$. const. für alle $E \geq 1$.

2. Es gibt ein $l>0$, so daß $\left\|R^{l} \phi R^{l}\right\|<\infty$.

Beweis. Die Richtung $1 \Rightarrow 2$ folgt durch Ausnutzen der Spektraldarstellung von $R^{l}$ - siehe Lemma 3.27 in Anhang $3 . \mathrm{C}$ (dabei ist $l=k+1$ ). Um die umgekehrte Richtung zu zeigen, berechnen wir

$$
\|P(E) \phi P(E)\| \leq\left\|P(E) R^{-l}\right\|\left\|R^{l} \phi R^{l}\right\|\left\|R^{-l} P(E)\right\| \leq E^{2 l} \cdot \text { const. }(E \geq 1),
$$

also folgt Aussage 1 aus 2 mit $k=2 l$.

Wir betrachten nun die Verbindung zwischen der Konvergenz im Phasenraumlimes $E r \rightarrow 0$ und der Konvergenz unter Energiedämpfung $R^{l} \cdot R^{l}$. 
Satz 3.10. Es sei $k>0, \psi \in \bar{\Psi}$ mit $\left\|R^{k} \psi_{\mathrm{R}} R^{k}\right\|<\infty$, $\rho$ eine Nullfolge, $\eta: \mathbb{R}^{+} \rightarrow \mathbb{R}^{+}$eine Funktion und $\lambda>0$, so daß

$$
\limsup _{E r \rightarrow 0} \frac{\|\psi\|_{E, r}}{E^{k} \eta(r)}=0 \quad \text { und } \quad \eta(r) \underset{\rho}{\gg r^{\lambda} .}
$$

Weiter sei $A_{r} \in \mathfrak{A}(r)(r \in \rho)$ eine Folge von Operatoren mit

$$
\left\|A_{r}\right\| \leq \frac{1}{\rho} \frac{1}{\eta(r)}
$$

Dann gibt es ein $l>0$, so daß

$$
\left\|R^{l} \psi_{R}\left(A_{r}\right) R^{l}\right\| \underset{\rho}{\rightarrow} 0
$$

Beweis. Für $r \in \rho$ setzen wir im folgenden $E_{r}=\frac{1}{\sqrt{r}}$, so daß dann $E_{r} \cdot r \rightarrow 0$. Im Limes $r \rightarrow 0$ haben wir den Ausdruck

$$
\left\|R^{l} \psi_{\mathrm{R}}\left(A_{r}\right) R^{l}\right\|
$$

zu kontrollieren, der zunächst wegen der polynomialen Hochenergieschranken von $\psi$ wohldefiniert ist (für $l \geq k$ ). Man erhält mit $Q(E):=1-P(E)$ durch Anwenden der Dreiecksungleichung:

$$
\begin{aligned}
\left\|R^{l} \psi_{\mathrm{R}}\left(A_{r}\right) R^{l}\right\| & \leq\left\|R^{l} Q\left(E_{r}\right) \psi_{\mathrm{R}}\left(A_{r}\right) R^{l}\right\|+\left\|R^{l} P\left(E_{r}\right) \psi_{\mathrm{R}}\left(A_{r}\right) Q\left(E_{r}\right) R^{l}\right\| \\
& +\left\|R^{l} P\left(E_{r}\right) \psi_{\mathrm{R}}\left(A_{r}\right) P\left(E_{r}\right) R^{l}\right\|
\end{aligned}
$$

Der erste Summand läßt sich dabei abschätzen durch

$$
\begin{aligned}
\left\|R^{l} Q\left(E_{r}\right) \psi_{\mathrm{R}}\left(A_{r}\right) R^{l}\right\| & \leq\left\|R^{l-k} Q\left(E_{r}\right)\right\|\left\|R^{k} \psi_{\mathrm{R}} R^{k}\right\|\left\|R^{l-k}\right\|\left\|A_{r}\right\| \\
& \leq\left(\frac{1}{1+E_{r}}\right)^{l-k} \frac{1}{\eta(r)} \cdot \text { const. } \leq\left(\frac{1}{1+\sqrt{r}}\right)^{l-k} r^{-\lambda} \cdot \text { const. } \underset{\rho}{\rightarrow} 0
\end{aligned}
$$

für genügend großes $l$. Der zweite Summand in (3.3.13) verschwindet im Limes analog. $\mathrm{Zu}$ zeigen bleibt, daß auch für den dritten Term gilt

$$
\left\|R^{l} P\left(E_{r}\right) \psi_{\mathrm{R}}\left(A_{r}\right) P\left(E_{r}\right) R^{l}\right\| \underset{\rho}{\rightarrow} 0 .
$$

Dazu betrachten wir die Operatoren $B_{r}:=P\left(E_{r}\right) \psi_{\mathrm{R}}\left(A_{r}\right) P\left(E_{r}\right)$. Es sei $E^{\prime} \geq 1$ beliebig. Mit $E_{\min }:=\min \left\{E_{r}, E^{\prime}\right\}$ ist dann

$$
P\left(E^{\prime}\right) B_{r} P\left(E^{\prime}\right) \in \Sigma_{E_{\min }}^{*}
$$

so daß

$$
\left\|P\left(E^{\prime}\right) B_{r} P\left(E^{\prime}\right)\right\| \leq\left\|\psi_{\mathrm{R}}\left(A_{r}\right)\right\|_{E_{\min }} \leq\left\|\psi_{\mathrm{R}}\right\|_{E_{\min }, r}\left\|A_{r}\right\| \leq \frac{1}{\eta(r)}\|\psi\|_{E_{\min }, r}
$$


Da nach Voraussetzung gilt

$$
\lim _{r \rightarrow 0} \frac{\|\psi\|_{E_{\min }, r}}{E_{\min }^{k} \eta(r)}=0 \quad\left(\text { denn } E_{\min } \cdot r \leq E_{r} \cdot r \rightarrow 0\right),
$$

wobei die Konvergenz gleichmäßig in $E_{\min } \leq E_{r}$ ist, können wir zu gegebenem $\epsilon>0$ ein $r_{(0)} \in \rho$ finden, so daß für $r \leq r_{(0)}$ gilt:

$$
\left\|P\left(E^{\prime}\right) B_{r} P\left(E^{\prime}\right)\right\| \leq \epsilon E_{\min }^{k} \leq \epsilon\left(E^{\prime}\right)^{k} .
$$

Dies gilt für alle $E^{\prime} \geq 1 ; \epsilon$ und $r_{(0)}$ sind von $E^{\prime}$ unabhängig. Nach Lemma 3.26 in Anhang $3 . \mathrm{C}$ können wir dann ein nicht von $\epsilon$ und $r$ abhängiges $d>0$ finden, so daß

$$
\left\|R^{k+1} B_{r} R^{k+1}\right\| \leq \epsilon \cdot d \quad \text { für } r \leq r_{(0)}, r \in \rho .
$$

Damit verschwindet auch dieser Term für $r \rightarrow 0$, womit der Satz bewiesen ist.

Konvergenz Nach diesen Vorbereitungen sind wir nun in der Lage, das Rechts-Bild einer gegebenen homogenen Approximation durch lokale Operatoren zu approximieren. Es sei dazu $\left(\rho, g,\left\{\psi_{j}\right\}_{j=1}^{J}\right)$ eine homogene Approximation mit polynomialen Energieschranken. Wir zeigen durch Induktion nach $K$ (für $K \leq J$ ), daß

$$
R^{l}\left(\bigoplus_{j=1}^{K} \operatorname{Bild}_{\mathrm{R}} \psi_{j}\right) R^{l}=\bigcap_{r>0}{\overline{R^{l} \mathfrak{A}(r) R^{l}}}^{\|\cdot\|}
$$

wobei $\ldots\|\cdot\|$ den Normabschluß bezeichnet.

Den Induktionsanfang machen wir bei $K=0$ (also mit der „trivialen Aussage“). Ist (3.3.21) für $K-1$ statt $K$ bereits bekannt, dann gehen wir so vor: Nach Voraussetzung gilt

$$
\limsup _{E r \rightarrow 0} \frac{\left\|\Xi-\oplus_{j=1}^{J} \psi_{j}\right\|_{E, r}}{g(E) r^{\bar{\gamma}-\epsilon}}=0,
$$

wobei wir $\epsilon>0$ so klein wählen können, daßt

$$
\eta_{K} \underset{\rho}{\geq} \eta_{J} \gg r^{\bar{\gamma}-\epsilon}
$$

Die Dreiecksungleichung liefert aufgrund der „asymptotischen Anordnung“ der $\eta_{j}$, daß

$$
\limsup _{E r \rightarrow \rho} \frac{\left\|\Xi-\oplus_{j=1}^{K} \psi_{j}\right\|_{E, r}}{g(E) \eta_{K}(r)}=0 .
$$

Wir können dabei ohne Einschränkung annehmen, daß $g(E)=E^{k}$ mit genügend großem $k>0$. Zu gegebenem $\phi \in \operatorname{Bild}_{\mathrm{R}} \psi_{K}$ verschaffen wir uns nun nach Satz 3.8 eine Folge $A_{r} \in \mathfrak{A}(r)(r \in \rho)$ und ein $c>0$, so daß

$$
\psi_{K}\left(A_{r}\right)=\phi, \quad\left\|A_{r}\right\| \leq \frac{c}{\eta_{K}(r)}
$$

\footnotetext{
${ }^{4}$ Die Funktionen $\eta_{j}$ werden hier wie in Definition 3.5 verwendet.
} 
Damit können wir Satz 3.10 anwenden; wir erhalten

$$
\left\|R^{l}\left(\Xi_{\mathrm{R}}\left(A_{r}\right)-\bigoplus_{j=1}^{K} \psi_{j, \mathrm{R}}\left(A_{r}\right)\right) R^{l}\right\| \underset{\rho}{\rightarrow} 0
$$

für große $l$. Nach Auswertung der Abbildungen bedeutet dies

$$
R^{l} \phi R^{l}=\underset{r \rightarrow 0}{\lim } \underset{r \rightarrow}{\lim }\left(R^{l} A_{r} R^{l}-\bigoplus_{j=1}^{K-1} \psi_{j, \mathrm{R}}\left(A_{r}\right)\right) .
$$

Nach Induktionsvoraussetzung ist $\psi_{j, \mathrm{R}}\left(A_{r}\right) \in \operatorname{Bild}_{\mathrm{R}} \psi_{j}$ für $j<K$ bereits durch beliebig gut lokalisierte Operatoren approximierbar; wir haben also für große $l$

$$
R^{l} \phi R^{l} \in \overline{R^{l} \mathfrak{A}(r) R^{l}+{\overline{R^{l} \mathfrak{A}(r) R^{l}}}^{\|\cdot\|}}\|\cdot\|{\overline{R^{l} \mathfrak{A}(r) R^{l}}}^{\|\cdot\|} \quad \forall r>0 .
$$

Damit haben wir insgesamt gezeigt:

Satz 3.11. Sei $\left(\rho, g,\left\{\psi_{j}\right\}\right)$ eine homogene Approximation mit polynomialen Energieschranken. Dann gilt für genügend große $l$ :

$$
R^{l}\left(\operatorname{Bild}_{\mathrm{R}} \psi_{j}\right) R^{l} \subset \bigcap_{r>0}{\overline{R^{l} \mathfrak{A}(r) R^{l}}}^{\|\cdot\|} \quad \forall j .
$$

Zusammenfassung Die Ergebnisse der vorangegangenen Abschnitte lassen sich folgendermaßen zusammenfassen: Wir waren ausgegangen von einer Theorie, die das asymptotische Phasenraumkriterium erfüllt; das hatte uns zur Definition der Räume $\Phi_{\bar{\gamma}}$ geführt (Satz 3.2). In diesen Räumen konnten wir eine Basis finden aus Elementen, die zu gewissen (,stabilen“) Nullfolgen $\rho$ assoziiert waren (Satz 3.4); die zugehörigen bilinearen Abbildungen konnten wir als homogene Approximationen genauer analysieren (Satz 3.6). Unter der zusätzlichen Voraussetzung polynomialer Energieschranken konnten wir das RechtsBild dieser Approximationen schließlich als Grenzwerte lokaler Operatoren darstellen (Satz 3.11). Wir erhalten also:

Korollar 3.12. Die betrachtete Theorie erfülle das asymptotische Phasenraumkriterium mit polynomialen Energieschranken. Dann gilt für die in Satz 3.9 beschriebenen Räume $\Phi_{\bar{\gamma}}$ :

$$
R^{l} \Phi_{\bar{\gamma}} R^{l} \subset \bigcap_{r>0}{\overline{R^{l} \mathfrak{A}(r) R^{l}}}^{\|\cdot\|} \quad \text { für } z u \bar{\gamma}>0 \text { ausreichend groß gewähltes } l \text {. }
$$

Diese Eigenschaft ist genau die, welche die Elemente der $\Phi_{\bar{\gamma}}$ zu lokalen Punktfeldern macht, wie wir unten noch sehen werden.

\subsection{Lokalitätseigenschaften}

Im letzten Abschnitt haben wir die Approximation der erhaltenen quadratischen Formen $\phi \in \Phi_{\bar{\gamma}}$ durch lokale Operatoren hergeleitet. Es liegt nahe, daß dies auch Lokalitätseigenschaften der quadratischen Formen $\phi$ nach sich zieht. Wir formulieren diese Eigenschaften 
nun genauer und untersuchen außerdem die Eigenschaften der $\phi$ als operatorwertige Distributionen (im Wightman-Sinn).

Dazu werden wir den Raum der von uns erhaltenen Felder mit dem von Fredenhagen und Hertel [FH81] betrachteten vergleichen. Dies verschafft uns neben Lokalitätsaussagen auch Einsicht in weitere Eigenschaften der betrachteten Felder.

Fredenhagen und Hertel betrachteten folgende Klasse von quadratischen Formen (und etablierten sie als lokale Punktfelder): :

$$
\Phi_{\mathrm{FH}}=\left\{\phi \in \bar{\Sigma}^{*} \mid \exists l>0:\left\|R^{l} \phi R^{l}\right\|<\infty, R^{l} \phi R^{l} \in \bigcap_{r>0}{\overline{R^{l} \mathfrak{A}(r) R^{l}}}^{w}\right\} .
$$

Die Autoren zeigten, daß $\Phi_{\mathrm{FH}}$ gerade die polynomial energiebeschränkten quadratischen Formen enthält, die einer sehr schwachen Kommutativitätsbedingung mit den lokalen Algebren genügen. Insofern kann $\Phi_{\mathrm{FH}}$ als Raum aller zum Netz $\mathfrak{A}$ assoziierten Punktfelder verstanden werden, wenn man die polynomialen Energieschranken als Obervoraussetzung betrachtet.

Nach den Ergebnissen des letzten Abschnitts ist sofort klar, daß unsere Felder die Bedingungen von Fredenhagen und Hertel erfüllen, daß also $\Phi_{\bar{\gamma}} \subset \Phi_{\mathrm{FH}}$ für alle $\bar{\gamma}>0$. Zunächst scheint - da in (3.4.1) der schwache und nicht der Normabschluß gewählt ist - die Klasse $\Phi_{\mathrm{FH}}$ aber echt größer zu sein. Im Laufe der Diskussion werden wir jedoch zeigen, daß die Vereinigung aller $\Phi_{\bar{\gamma}}$ den Raum $\Phi_{\mathrm{FH}}$ tatsächlich ausschöpft, daß also unser Konstruktionsverfahren sämtliche Fredenhagen-Hertel-Felder liefert.

Die dabei verwendeten Methoden lehnen sich zum Teil sehr dicht an [FH81] an; wir reproduzieren sie hier der Vollständigkeit halber.

Distributionen Wir beschäftigen uns zunächst mit den Eigenschaften der quadratischen Formen auf $\Phi_{\mathrm{FH}}$ als Distributionen (durch „Ausschmieren“ mit einer Testfunktion). Sei $\phi \in \bar{\Sigma}^{*}$ fest, und es gelte $\left\|R^{l} \phi R^{l}\right\|<\infty$ für große $l$. (Die andere Fredenhagen-HertelBedingung benötigen wir zunächst nicht.) Für $f \in \mathcal{S}(\mathcal{M})$ definieren wir

$$
\phi(f):=\int d^{s+1} x f(x) U(x) \phi U(x)^{*}
$$

zunächst als Linearform auf $\bar{\Sigma}$, d.h. im Sinne eines schwachen Integrals. Die Form $\phi(f)$ ist durch die Integration jedoch soweit „regularisiert“, daß sie sich zu einem (im allgemeinen unbeschränkten) Operator erweitern läßt, und zwar auf dem Definitionsbereich

$$
\mathcal{C}^{\infty}(\mathcal{H}):=\bigcap_{l \in \mathbb{N}} R^{l} \mathcal{H}
$$

Um dies zu sehen, verwenden wir die in Lemma 3.28 in Anhang $3 . \mathrm{C}$ berechneten Vertauschungsrelation

$$
[R, \phi(f)]=-i R \phi\left(\partial_{t} f\right) R
$$

\footnotetext{
${ }^{5}$ Schreiben wir im folgenden $\left\|R^{l} \phi R^{l}\right\|<\infty$, so ist damit immer gemeint, daß sich die Linearform $R^{l} \phi R^{l} \in \bar{\Sigma}^{*} \mathrm{zu}$ einem beschränkten Operator erweitern läßt. Da diese Erweiterung dann eindeutig ist, verzichten wir auf eine neue Bezeichnung.
} 
mit ihrer Hilfe erhält man aus $\left\|R^{l} \phi(f) R^{l}\right\|$ (was für große $l$ aus der Konstruktion klar ist) sofort $\left\|\phi(f) R^{2 l}\right\|<\infty$. Für $\xi \in \mathcal{C}^{\infty}(\mathcal{H})$ ist also die Abbildung

$$
\chi \mapsto(\chi|\phi(f)| \xi)
$$

stetig und wird daher vom Skalarprodukt mit einem Vektor $\phi(f) \xi \in \mathcal{H}$ induziert. Eine erneute Anwendung der Vertauschungsrelation (3.4.4) liefert $\phi(f) \xi \in \mathcal{C}^{\infty}(\mathcal{H})$, d.h. der Operator $\phi(f)$ überführt seinen Definitionsbereich $\mathcal{C}^{\infty}(\mathcal{H})$ in sich.

Mit $\left\|R^{l} \phi R^{l}\right\|<\infty$ gilt auch $\left\|R^{l} \phi^{*} R^{l}\right\|<\infty$, wenn $\phi^{*}$ die zu $\phi$ adjungierte quadratische Form bezeichnet; damit existiert auch $\phi^{*}(f)$. Für $\xi, \xi^{\prime} \in \mathcal{H}_{E}$ ist sofort klar, daß

$$
\left(\xi \mid \phi(f) \xi^{\prime}\right)=\overline{\left(\xi^{\prime} \mid \phi^{*}(\bar{f}) \xi\right)}
$$

diese Relation (die definierende Gleichung für $\left.\phi(f)^{*}\right)$ läßt sich auf Vektoren $\xi \in \mathcal{C}^{\infty}(\mathcal{H}), \xi^{\prime} \in$ $\mathcal{H}$ stetig erweitern, deshalb ist

$$
\phi^{*}(\bar{f}) \subset \phi(f)^{*}
$$

und $\phi(f)^{*}$ ist dicht definiert. Damit ist $\phi(f) \subset \phi(f)^{* *}$ abschließbar [Kat84, Ch. III Thm. $5.28]$.

Die Abbildung $f \mapsto \phi(f)$ ist nach obigen Ergebnissen eine operatorwertige Distribution auf $\mathcal{C}^{\infty}(\mathcal{H})$; diese ist sogar temperiert, denn für festes $\chi, \xi \in \mathcal{C}^{\infty}(\mathcal{H})$ und genügend großes $l$ gilt

$$
\begin{aligned}
|(\chi \mid \phi(f) \xi)| \leq \int d^{s+1} x|f(x)| \mid & \left(\chi \mid U(x) \phi U(x)^{*} \xi\right) \mid \\
& \leq\left\|R^{-l} \chi\right\|\left\|R^{-l} \xi\right\|\left\|R^{l} \phi R^{l}\right\| \int|f(x)| d^{s+1} x \rightarrow 0,
\end{aligned}
$$

wenn $f \rightarrow 0$ in der $\mathcal{S}$-Topologie. Damit haben wir folgendes Resultat:

Satz 3.13. Sei $\phi \in \bar{\Sigma}^{*}$, und es gelte $\left\|R^{l} \phi R^{l}\right\|<\infty$ für große l. Dann läßt sich die Linearform

$$
\phi(f):=\int d^{s+1} x f(x) U(x) \phi U(-x) \quad(f \in \mathcal{S}(\mathcal{M}))
$$

zu einem abschließbaren Operator mit dichtem Definitionsbereich

$$
\mathcal{C}^{\infty}(\mathcal{H})=\bigcap_{l \in \mathbb{N}} R^{l} \mathcal{H}
$$

erweitern. Es gilt $\phi(f) \mathcal{C}^{\infty}(\mathcal{H}) \subset \mathcal{C}^{\infty}(\mathcal{H})$. Die Abbildung $f \mapsto \phi(f)$ ist eine temperierte operatorwertige Distribution.

Insbesondere erlaubt uns dieser Satz, Produkte von Operatoren $\phi(f) \phi^{\prime}(g)$ für $f, g \in$ $\mathcal{S}(\mathcal{M})$ und $\phi, \phi^{\prime} \in \Phi_{\mathrm{FH}}$ zu betrachten.

Intuitiv ist klar, daß man $\phi$ aus den Operatoren $\phi(f)$,zurückgewinnen“ kann, indem man für $f$ eine Delta-Folge $f_{n}$ einsetzt. Die Details zeigt folgendes Lemma:

\footnotetext{
${ }^{6} \mathrm{Zu}$ Konvergenzfragen des auftretenden Integrals siehe (3.C.10).
} 
Lemma 3.14. Sei $\phi \in \Phi_{\mathrm{FH}}$ mit $\left\|R^{l} \phi R^{l}\right\|<\infty$. Sei ferner $\left(f_{n}\right) \subset \mathcal{S}(\mathcal{M})$ eine Folge mit

$$
f_{n}(x) \geq 0, \quad \int f_{n}(x) d^{s+1} x=1 \forall n \quad \text { und } \quad \operatorname{supp} f_{n} \subset \mathcal{O}_{r_{n}} \text { mit } r_{n} \rightarrow 0 .
$$

Dann gilt

$$
\begin{aligned}
& R^{l} \phi\left(f_{n}\right) R^{l} \underset{s}{\longrightarrow} R^{l} \phi R^{l}, \\
& R^{l+1} \phi\left(f_{n}\right) R^{l+1} \underset{\|\cdot\|}{\longrightarrow} R^{l+1} \phi R^{l+1}, \quad \text { genauer: }\left\|R^{l+1}\left(\phi\left(f_{n}\right)-\phi\right) R^{l+1}\right\| \leq r_{n} \cdot \text { const. }(\phi) .
\end{aligned}
$$

Beweis. Für festes $\xi \in \mathcal{H}$ hat man

$$
\begin{aligned}
\left.\| R^{l}\left(\phi\left(f_{n}\right)-\phi\right) R^{l}\right) \xi\left\|\leq \int d^{s+1} x f_{n}(x)\right\| R^{l}\left(U(x) \phi U(x)^{*}-\phi\right) R^{l} \xi \| \\
\leq \sup _{x \in \mathcal{O}_{r_{n}}}\left\|U(x) R^{l} \phi R^{l}\left(U(x)^{*}-\mathbf{1}\right) \xi\right\|+\sup _{x \in \mathcal{O}_{r_{n}}}\left\|(U(x)-\mathbf{1}) R^{l} \phi R^{l} \xi\right\| .
\end{aligned}
$$

Dann gilt für den ersten Summanden (der zweite wird analog behandelt):

$$
\sup _{x \in \mathcal{O}_{r_{n}}}\left\|U(x) R^{l} \phi R^{l}\left(U(x)^{*}-\mathbf{1}\right) \xi\right\| \leq\left\|R^{l} \phi R^{l}\right\| \sup _{x \in \mathcal{O}_{r_{n}}}\|(U(-x)-\mathbf{1}) \xi\|,
$$

was wegen der starken Stetigkeit der Translationen für $n \rightarrow \infty$ verschwindet. Die starke Konvergenz ist damit gezeigt.

Zum Beweis der behaupteten Normkonvergenz berechnet man analog

$$
\begin{aligned}
& \left.\| R^{l+1}\left(\phi\left(f_{n}\right)-\phi\right) R^{l+1}\right) \| \\
& \quad \leq \sup _{x \in \mathcal{O}_{r_{n}}}\left\|U(x) R^{l+1} \phi R^{l} R\left(U(x)^{*}-\mathbf{1}\right)\right\|+\sup _{x \in \mathcal{O}_{r_{n}}}\left\|(U(x)-\mathbf{1}) R R^{l} \phi R^{l+1}\right\| .
\end{aligned}
$$

Daraus folgt sicher die behauptete Abschätzung, wenn noch gezeigt werden kann, daß

$$
\|(U(x)-\mathbf{1}) R\| \leq\|x\| \cdot \text { const. }
$$

Um diese Relation einzusehen, berechnet man mit Hilfe der gemeinsamen Spektraldarstellung der Translationsoperatoren, daß

$$
\|(U(x)-\mathbf{1}) R\|=\sup _{p \in \bar{V}_{+}}\left|\frac{e^{i p_{\mu} x^{\mu}}-1}{1+p_{0}}\right| .
$$

Wegen der Gültigkeit der Abschätzung

$$
\left|e^{i p_{\mu} x^{\mu}}-1\right| \leq\left|\sin \left(p_{\mu} x^{\mu}\right)\right|+\left|\cos \left(p_{\mu} x^{\mu}\right)-1\right| \leq 2\left|p_{\mu} x^{\mu}\right| \leq 8 p_{0}\|x\| \quad\left(p \in \bar{V}_{+}\right)
$$

folgt dann

$$
\|(U(x)-\mathbf{1}) R\| \leq\|x\| \cdot \sup _{p_{0} \geq 0} \frac{8 p_{0}}{1+p_{0}}
$$

womit das Lemma bewiesen ist. 
Lokalität Wir werten nun die Lokalitätseigenschaften der Felder $\phi \in \Phi_{\mathrm{FH}}$ aus, d.h. die geforderte Approximierbarkeit durch lokale Operatoren. Zunächst sind die $\phi$ relativ lokal zueinander:

Satz 3.15. Seien $\phi, \phi^{\prime} \in \Phi_{\mathrm{FH}}$ und $f, g \in \mathcal{S}(\mathcal{M})$; dabei seien $\operatorname{supp} f$ und $\operatorname{supp} g$ raumartig getrennt. Dann gilt

$$
\left[\phi(f), \phi^{\prime}(g)\right]=0
$$

als Operatorgleichung auf $\mathcal{C}^{\infty}(\mathcal{H})$.

Beweis. Wir nehmen zunächst an, daß supp $f$ kompakt ist. Dann kann man eine Nullumgebung $\mathcal{O}$ finden, so daß auch $\operatorname{supp} f+\mathcal{O}$ und $\operatorname{supp} g+\mathcal{O}$ raumartig getrennt sind. Wir wählen Netze $A_{\lambda}$ und $B_{\mu}$ in $\mathfrak{A}(\mathcal{O})$, so daß

$$
R^{l} A_{\lambda} R^{l} \underset{w}{\rightarrow} R^{l} \phi R^{l}, \quad R^{l} B_{\mu} R^{l} \underset{w}{\rightarrow} R^{l} \phi^{\prime} R^{l} .
$$

Dann gilt auch

$$
R^{l} A_{\lambda}(f) R^{l} \underset{w}{\rightarrow} R^{l} \phi(f) R^{l}, \quad R^{l} B_{\mu}(g) R^{l} \underset{w}{\rightarrow} R^{l} \phi^{\prime}(g) R^{l},
$$

denn im Ausdruck

$$
\left(\xi \mid R^{l} \phi(f) R^{l} \xi^{\prime}\right)=\int d^{s+1} x f(x) \lim _{\lambda}\left(\xi \mid U(x) R^{l} A_{\lambda} R^{l} U(x)^{*} \xi^{\prime}\right) \quad\left(\xi, \xi^{\prime} \in \mathcal{H}\right)
$$

können Limes und Integration vertauscht werden, da der Integrand gleichmäßig beschränkt ist (analog für $B_{\mu}$ ). Man hat dann für $\xi, \xi^{\prime} \in \mathcal{C}^{\infty}(\mathcal{H})$ :

$$
\left(\xi \mid\left[\phi(f), \phi^{\prime}(g)\right] \xi^{\prime}\right)=\lim _{\lambda} \lim _{\mu}\left(\xi \mid\left[A_{\lambda}(f), B_{\mu}(g)\right] \xi^{\prime}\right)=0,
$$

denn $A_{\lambda}(f)$ liegt in $\mathfrak{A}(\mathcal{O}+\operatorname{supp} f), B_{\mu}(g)$ in $\mathfrak{A}(\mathcal{O}+\operatorname{supp} g)$.

Für den Fall, daß supp $f$ nicht kompakt ist, führt eine Zerlegung $f=\sum f_{i}$ in Testfunktionen mit kompaktem Träger supp $f_{i} \subset \operatorname{supp} f$ zum Ziel.

Dies zeigt die relative Lokalität der Felder untereinander. Wie zu erwarten, erhält man ähnliche Lokalitätseigenschaften auch zwischen einem Feld und Elementen der lokalen Algebren $\mathfrak{A}(r)$. Allerdings sollen diese Eigenschaften etwas anders formuliert werden. Wir sagen, ein abgeschlossener Operator $C$ mit Definitionsbereich $\mathcal{D}_{C} \subset \mathcal{H}$ ist affiliiert zur von-Neumann-Algebra $\mathfrak{A}(r)$, wenn für $A^{\prime} \in \mathfrak{A}(r)^{\prime}$ stets gilt

$$
\begin{aligned}
A^{\prime} \mathcal{D}_{C} & =\mathcal{D}_{C} \\
\text { und } \quad\left[C, A^{\prime}\right] & =0 \quad \text { als Gleichung auf } \mathcal{D}_{C} .
\end{aligned}
$$

Wir notieren dies als $C \eta \mathfrak{A}(r)$.

In unserem Fall gilt:

Lemma 3.16. Sei $\phi \in \Phi_{\mathrm{FH}}$, und seien $f \in \mathcal{S}(\mathcal{M}), r>0$ gegeben, so daß supp $f \subset \mathcal{O}_{r}$. Dann gilt für den Abschluß $\phi(f)^{-}$von $\phi(f)$ :

$$
\phi(f)^{-} \eta \mathfrak{A}(r) .
$$


Beweis. Der Träger von $f$ liegt kompakt in der offenen Menge $\mathcal{O}_{r}$, also können wir Radien $r^{\prime}, r^{\prime \prime}>0$ wählen, so daß $\operatorname{supp} f+\mathcal{O}_{r^{\prime}}+\mathcal{O}_{r^{\prime \prime}} \subset \mathcal{O}_{r}$. Wir wählen eine nichtnegative Testfunktion $g$ mit Träger in $\mathcal{O}_{r^{\prime}}$ und $\int g(x) d^{s+1} x=1$; setzen wir dann $g_{n}:=n^{s+1} g\left(n^{-1} x\right)$, dann gilt auch $\int g_{n}(x) d^{s+1} x=1$.

Ist nun $A^{\prime} \in \mathfrak{A}(r)^{\prime}$, dann erfüllt $A^{\prime}$ als beschränkter Operator insbesondere die Bedingungen von Satz 3.13 mit $l=0$, und es ist deshalb $A^{\prime}\left(g_{n}\right) \mathcal{C}^{\infty}(\mathcal{H}) \subset \mathcal{C}^{\infty}(\mathcal{H})$. Eine analoge Überlegung wie im Beweis zu Satz 3.15 zeigt

$$
\left[\phi(f), A^{\prime}\left(g_{n}\right)\right]=0 \forall n \quad \text { als Gleichung auf } \mathcal{C}^{\infty}(\mathcal{H})
$$

wobei die Lokalisierung von $\phi(f)$ und $A^{\prime}\left(g_{n}\right)$ sowie deren „Trennung“ durch einen weiteren Doppelkegel $\mathcal{O}_{r^{\prime \prime}}$ ausgenutzt wird.

Es bezeichne $\mathcal{D}^{-}$den Definitionsbereich von $\phi(f)^{-}$; zu $\xi \in \mathcal{D}^{-}$gibt es also eine Folge $\left(\xi_{n}\right) \subset \mathcal{C}^{\infty}(\mathcal{H})$ mit

$$
\xi_{n} \rightarrow \xi, \quad \phi(f) \xi_{n} \rightarrow \phi(f)^{-} \xi
$$

Nun gilt

$$
\begin{aligned}
\left\|A^{\prime}\left(g_{n}\right) \xi_{n}-A^{\prime} \xi\right\| \leq\left\|A^{\prime}\left(g_{n}\right)\right\|\left\|\left(\xi_{n}-\xi\right)\right\| & +\left\|\left(A^{\prime}-A^{\prime}\left(g_{n}\right)\right) \xi\right\| \\
& \leq\left\|A^{\prime}\right\|\left\|\left(\xi_{n}-\xi\right)\right\|+\left\|\left(A^{\prime}-A^{\prime}\left(g_{n}\right)\right) \xi\right\| .
\end{aligned}
$$

Da $\xi_{n} \rightarrow \xi$ und nach Lemma 3.14 auch $A^{\prime}\left(g_{n}\right) \underset{s}{\rightarrow} A^{\prime}$, erhalten wir also

$$
A^{\prime}\left(g_{n}\right) \xi_{n} \rightarrow A^{\prime} \xi
$$

Ganz analog folgt

$$
A^{\prime}\left(g_{n}\right) \phi(f) \xi_{n} \rightarrow A^{\prime} \phi(f)^{-} \xi
$$

die Vertauschungsrelation (3.4.22) liefert also

$$
\phi(f) A^{\prime}\left(g_{n}\right) \xi_{n} \rightarrow A^{\prime} \phi(f)^{-} \xi,
$$

und wegen der Abgeschlossenheit von $\phi(f)^{-}$bedeutet das

$$
A^{\prime} \xi \in \mathcal{D}^{-} \quad \text { und } \quad \phi(f)^{-} A^{\prime} \xi=A^{\prime} \phi(f)^{-} \xi
$$

Wir wissen also, daß $\phi(f)^{-} \eta \mathfrak{A}(r)$. Für den Fall beschränkter $\phi(f)^{-}=\phi(f)$ hieße dies $\phi(f) \in \mathfrak{A}(r)^{\prime \prime}=\mathfrak{A}(r)$. Für unbeschränkte $\phi(f)$ kann das so natürlich nicht gelten; ist aber

$$
\phi(f)^{-}=V\left|\phi(f)^{-}\right|=V \int_{0}^{\infty} \lambda d E(\lambda)
$$

die Polarzerlegung von $\phi(f)^{-}$[Kat84, Ch. VI $\S 2.7$ ], dann gilt stets $V \in \mathfrak{A}(r), E(\lambda) \in \mathfrak{A}(r)$ - zum Beweis siehe [BR79, Lemma 2.5.8]. 
Lokale Approximation Wir zeigen nun, daß sich die quadratischen Formen aus $\Phi_{\mathrm{FH}}$ aufgrund der gewonnenen Erkenntnisse noch besser durch lokale Operatoren approximieren lassen, als wir dies in der Definition (3.4.1) gefordert hatten, nämlich (unter Energiedämpfung) in der Operatornorm, wobei auch die Norm der approximierenden Operatoren kontrolliert werden kann.

Satz 3.17. Sei $\phi \in \Phi_{\mathrm{FH}}$. Dann gibt es eine Folge $A_{r} \in \mathfrak{A}(r)(0<r<1)$, ein $l>0$ und ein $\kappa>0$, so daß

$$
\left\|R^{l}\left(A_{r}-\phi\right) R^{l}\right\| \leq r \cdot \text { const. }, \quad\left\|A_{r}\right\| \leq r^{-\kappa} \cdot \text { const. }
$$

Beweis. Es sei $f \in \mathcal{S}(\mathcal{M})$ eine feste Testfunktion mit $f(x) \geq 0, \int f(x) d^{s+1} x=1, \operatorname{supp} f \subset$ $\mathcal{O}_{r=1}$. Wir setzen

$$
f_{r}=r^{-(s+1)} f\left(\frac{x}{r}\right)
$$

so daß dann supp $f_{r} \subset \mathcal{O}_{r}$ und ebenfalls $\int f_{r}(x) d^{s+1} x=1$. Zunächst sei $r$ fest. Wir wissen, daß $\phi\left(f_{r}\right)^{-}$affiliiert zu $\mathfrak{A}(r)$ ist, und schreiben die Polarzerlegung als

$$
\begin{aligned}
\phi\left(f_{r}\right)=V_{r} \cdot D_{r} \quad \text { mit } & V_{r} \in \mathfrak{A}(r), D_{r}^{*}=D_{r}, D_{r} \geq 0, \\
& g\left(D_{r}\right) \in \mathfrak{A}(r) \text { für jede beschränkte Funktion } g .
\end{aligned}
$$

Wir setzen für $\epsilon>0$

$$
A_{r, \epsilon}=\frac{1}{\epsilon} V_{r} \sin \left(\epsilon D_{r}\right) \in \mathfrak{A}(r)
$$

und schätzen im folgenden die Norm von $R^{4 l}\left(A_{r, \epsilon}-\phi\left(f_{r}\right)\right) R^{4 l}$ ab. (Dabei sei $l$ so groß, daß $\left\|R^{l} \phi R^{l}\right\|<\infty$.) Man hat

$$
\left\|\left(A_{r, \epsilon}-\phi\left(f_{r}\right)\right) R^{4 l}\right\|=\left\|V_{r}\left(\frac{1}{\epsilon} \sin \left(\epsilon D_{r}\right)-D_{r}\right) R^{4 l}\right\| \leq\left\|\left(D_{r}-\frac{1}{\epsilon} \sin \left(\epsilon D_{r}\right)\right) R^{4 l}\right\| .
$$

Aus der leicht nachzuprüfenden Ungleichung

$$
|x-\sin x| \leq x^{2} \quad \forall x \geq 0
$$

erhält man durch Ersetzung $x \rightarrow \epsilon x$ :

$$
\left|x-\frac{1}{\epsilon} \sin \epsilon x\right| \leq \epsilon x^{2} \quad \forall x \geq 0, \epsilon>0 .
$$

Entsprechendes gilt für die quadrierte Ungleichung; daher folgt

$$
\left\|\left(D_{r}-\frac{1}{\epsilon} \sin \left(\epsilon D_{r}\right)\right) R^{4 l}\right\| \leq \epsilon\left\|D_{r}^{2} R^{4 l}\right\|=\epsilon\left\|\phi\left(f_{r}\right)^{*} \phi\left(f_{r}\right) R^{4 l}\right\| .
$$

Durch 4l-maliges Ausnutzen der aus Lemma 3.28 in Anhang 3.0 folgenden Identität

$$
\left[R, \phi\left(f_{r}\right)\right]=-i R \phi\left(\partial_{t} f_{r}\right) R=-\frac{i}{r} R \phi\left(r^{-(s+1)}\left(\partial_{t} f\right)\left(\frac{x}{r}\right)\right) R
$$


(bzw. analog für $\left.\phi\left(f_{r}\right)^{*}\right)$ und unter Verwendung der Abschätzung

$$
\left\|R^{l} \phi(\hat{f})^{(*)} R^{l}\right\| \leq \int|\hat{f}(x)| d^{s+1} x \cdot \text { const. } \forall \hat{f} \in \mathcal{S}(\mathcal{M})
$$

erhält man

$$
\left\|\phi\left(f_{r}\right)^{*} \phi\left(f_{r}\right) R^{4 l}\right\| \leq r^{-4 l} \cdot c,
$$

wobei $c>0$ nicht von $r$ abhängt. Damit ist schließlich

$$
\left\|R^{4 l}\left(A_{r, \epsilon}-\phi\left(f_{r}\right)\right) R^{4 l}\right\| \leq r^{-4 l} \cdot \epsilon \cdot c .
$$

Nun müssen wir noch die Konvergenz „, $\phi\left(f_{r}\right) \rightarrow \phi(0)$ “ betrachten. Lemma 3.14 liefert

$$
\left\|R^{l+1}\left(\phi\left(f_{r}\right)-\phi(0)\right) R^{l+1}\right\| \leq c^{\prime} \cdot r, \quad c^{\prime}>0 \text { konstant }
$$

so daß wir zusammen mit (3.4.40) erhalten (wobei $l>1$ angenommen werden darf):

$$
\left\|R^{4 l}\left(A_{r, \epsilon}-\phi\right) R^{4 l}\right\| \leq\left(\epsilon r^{-4 l}+r\right) \cdot c^{\prime \prime}, \quad c^{\prime \prime}>0 \text { konstant. }
$$

Setzen wir nun $\epsilon=r^{4 l+1}$, dann erhalten wir

$$
R^{4 l} A_{r, \epsilon} R^{4 l} \underset{r \rightarrow 0}{\stackrel{\|\cdot\|}{\longrightarrow}} R^{4 l} \phi R^{4 l}, \quad\left\|A_{r, \epsilon}\right\| \leq r^{-(4 l+1)} \cdot \text { const. }
$$

Damit ist die gewünschte Folge $A_{r} \equiv A_{r, \epsilon}$ gefunden.

Die Wahl der „Approximationsgüte“ $r$ c const. für die Norm der Differenzfolge ist hier willkürlich; durch eine Neuzuordnung der Folgenglieder, etwa $A_{r}^{\prime}:=A_{r^{\lambda}}(\lambda \geq 1)$ läßt sich genauso $\left\|R^{l}\left(A_{r}^{\prime}-\phi\right) R^{l}\right\| \leq r^{\lambda}$. const. für große $l$ erreichen, wobei die Normschranken der $A_{r}^{\prime}$ entsprechend skalieren. Wir können sogar noch weitere Eigenschaften der approximierenden Folgen fordern:

Satz 3.18. Die Operatoren $A_{r}$ in Satz 3.17 können so gewählt werden, daß mit einer geeigneten Testfunktion $f \in \mathcal{S}(\mathcal{M})$ und $f_{r}:=r^{-(s+1)} f\left(\frac{x}{r}\right)$ gilt:

$$
A_{r}\left(f_{r}\right) \in \mathfrak{A}(r), \quad\left\|A_{r}\left(f_{r}\right)\right\| \leq r^{-\kappa} \cdot \text { const. }, \quad\left\|R^{l}\left(A_{r}\left(f_{r}\right)-\phi\right) R^{l}\right\| \leq r \cdot \text { const. }
$$

Dieser Zusatz ist für spätere Anwendungen wichtig, da wir nun die Vertauschungsrelation aus Lemma 3.28 auf die approximierenden Folgen anwenden können.

Beweis. Durch Skalierung können wir annehmen, daß in Satz 3.17 schon $A_{r} \in \mathfrak{A}\left(\frac{r}{2}\right)$ gilt. Wir wählen eine Testfunktion $f \geq 0$ mit kompaktem Träger so, daß $\int f(x) d^{s+1} x=1$. Ist $f$ genügend gut lokalisiert, dann gilt sicher $A_{r}\left(f_{r}\right) \in \mathfrak{A}(r)$. Die Eigenschaft

$$
\left\|A_{r}\left(f_{r}\right)\right\| \leq\left\|A_{r}\right\|\left\|f_{r}\right\|_{1} \leq r^{-\kappa} \cdot \text { const. }
$$

folgt sofort aus der entsprechenden für $A_{r}$. Ebenso gilt

$$
\left\|R^{l}\left(\phi\left(f_{r}\right)-A_{r}\left(f_{r}\right)\right) R^{l}\right\| \leq\left\|f_{r}\right\|_{1} \cdot\left\|R^{l}\left(\phi-A_{r}\right) R^{l}\right\| \leq r \cdot \text { const. }
$$

Aus Lemma 3.14 erhalten wir

$$
\left\|R^{l}\left(\phi\left(f_{r}\right)-\phi\right) R^{l}\right\| \leq r \cdot \text { const. }
$$

nachdem $l$ eventuell vergrößert wurde. (3.4.45) und (3.4.46) liefern zusammen die dritte der behaupteten Relationen. 
Aufgrund der ,verbesserten“ Approximationseigenschaften können wir nun die Fredenhagen-Hertel-Felder mit unserem Phasenraumkriterium in Verbindung bringen. Wir betrachten im folgenden eine Theorie, die dem asymptotischen Phasenraumkriterium mit polynomialen Energieschranken genügt. Zu einem festen $\phi \in \Phi_{\mathrm{FH}}$ verschaffen wir uns nach Satz 3.17 eine Folge $A_{r} \in \mathfrak{A}(r)$, so daß

$$
\left\|A_{r}\right\| \leq r^{-\bar{\gamma}} \cdot \text { const. mit gewissem } \bar{\gamma}>0 ; \quad R^{l} A_{r} R^{l} \underset{n}{\rightarrow} R^{l} \phi R^{l} \text { für große } l>0 .
$$

Wir werden dann zeigen, daß $\phi \in \Phi_{\bar{\gamma}+1}$. Dazu verschaffen wir uns nach Satz 3.6 eine homogene Approximation $\left(\rho, g,\left\{\psi_{j}\right\}\right)$ zur Genauigkeit $\bar{\gamma}+1$. Wir setzen $\psi:=\oplus \psi_{j}$. Dann gilt

$$
\limsup _{E r \rightarrow 0} \frac{\|\Xi-\psi\|_{E, r}}{g(E) r^{\bar{\gamma}+1-\epsilon}}=0 \quad \forall \epsilon>0 \quad \text { und } \quad \operatorname{Bild}_{\mathrm{R}} \psi \subset \Phi_{\bar{\gamma}+1}
$$

Wir wählen $\epsilon=\frac{1}{2}$. Weiter sei $E \geq 1$ fest. Dann ist

$$
\lim _{r \rightarrow 0} \frac{\|\Xi-\psi\|_{E, r}}{r^{\bar{\gamma}+\frac{1}{2}}}=0
$$

insbesondere

$$
\lim _{\substack{r \rightarrow 0 \\ \rho}}\left\|(\Xi-\psi)\left(A_{r}\right)\right\|_{E} \leq \lim _{\substack{\rho \\ \rho}}\|(\Xi-\psi)\|_{E, r}\left\|A_{r}\right\|=0 .
$$

Zusammen mit (3.4.47) ergibt das

$$
\psi_{\mathrm{R}}\left(A_{r}\right) \rightarrow \phi \quad \text { in } \Sigma(E)^{*} .
$$

Andererseits bewegt sich die linke Seite im endlichdimensionalen Teilraum $P(E)\left(\operatorname{Bild}_{\mathrm{R}} \psi\right) P(E) \subset \Sigma(E)^{*}$; dieser ist abgeschlossen, also ist

$$
P(E) \phi P(E) \in P(E)\left(\operatorname{Bild}_{\mathrm{R}} \psi\right) P(E) .
$$

Dies gilt für alle $E \geq 1$, weshalb

$$
\phi \in \operatorname{Bild}_{\mathrm{R}} \psi \subset \Phi_{\bar{\gamma}+1},
$$

wie zuvor behauptet. Dieses Verfahren läßt sich für jedes $\phi \in \Phi_{\mathrm{FH}}$ durchführen (mit jeweils angepaßtem $\bar{\gamma}$ ); daher gilt:

Satz 3.19. Die betrachtete Theorie erfülle das asymptotische Phasenraumkriterium mit polynomialen Energieschranken. Dann ist

$$
\Phi_{\mathrm{FH}}=\bigcup_{\bar{\gamma}>0} \Phi_{\bar{\gamma}} .
$$

Der von uns bestimmte Feldinhalt stimmt also mit dem von Fredenhagen und Hertel betrachteten überein; wir erhalten durch unsere Konstruktion alle polynomial energiebeschränkten Punktfelder der Theorie. 
Zusammenfassung Wir haben in diesem Abschnitt verschiedene Eigenschaften der Fredenhagen-Hertel-Felder hergeleitet und konnten schließlich feststellen, daß die von uns konstruierte Klasse von Feldern mit der von Fredenhagen und Hertel betrachteten übereinstimmt. Wir stellen nun noch einmal zusammen, was wir im Laufe der Konstruktion also für unsere Punktfelder etabliert haben:

- Die $\phi \in \Phi_{\bar{\gamma}}$ werden durch Integration zu operatorwertigen temperierten Distributionen $f \mapsto \phi(f)$. Die Operatoren $\phi(f)$ sind abschließbar und besitzen den gemeinsamen invarianten dichten Definitionsbereich $\mathcal{C}^{\infty}(\mathcal{H})$.

- Die $\phi(f)$ sind lokal und relativ lokal; ihr Abschluß $\phi(f)^{-}$ist affiliiert zu den lokalen Algebren.

- Jedes $\phi \in \Phi_{\bar{\gamma}}$ läßt sich (unter Energiedämpfung) in der Operatornorm durch eine Folge $A_{r} \in \mathfrak{A}(r)$ approximieren. Die Norm der $A_{r}$ divergiert im Limes $r \rightarrow 0$ höchstens wie eine inverse Potenz.

\section{A Lokalisierte Zustände im Limes kleiner Abstände}

Wir untersuchen in diesem Abschnitt das Kurzabstandsverhalten von Räumen lokalisierter Zustände, d.h. endlichdimensionaler Teilräume $S \subset \overline{\mathfrak{A}}_{*}$, die in der Anwendung als LinksBilder gewisser Abbildungen von endlichem Rang auftreten. Wir wollen eine Zerlegung von $S$ in eine Summe von Räumen herleiten, deren Elemente sich im Kurzabstandslimes jeweils gleich verhalten.

Wir betrachten zunächst den einfachsten Fall, nämlich den, in dem alle Elemente von $S$ dieselbe Asymptotik zeigen:

Definition 3.20. Ein endlichdimensionaler Teilraum $S \subset \overline{\mathfrak{A}}_{*}$ heiße homogen bezüglich einer Nullfolge $\rho$, wenn es eine Funktion $\eta$ auf $(0,1]$ gibt, so daß für alle $\sigma \in S \backslash\{0\}$

$$
\|\sigma\|_{r} \underset{\rho}{\sim} \eta(r)
$$

Wir schreiben in diesem Fall auch kurz $S \underset{\rho}{\sim} \eta$

Auf homogenen Teilräumen von $\overline{\mathfrak{A}}_{*}$ läßt sich das $r$-Verhalten der Zustände sogar gleichmäßig über den ganzen Raum abschätzen, wie der folgende Satz zeigt.

Satz 3.21. Sei $S \subset \overline{\mathfrak{A}}_{*}$ homogener Teilraum bezüglich $\rho(S \underset{\rho}{\sim} \eta)$, und sei $\|\cdot\|_{S}$ eine Norm auf $S$ (als endlichdimensionalem Raum). Dann gibt es eine Konstante $c>0$, so daß

$$
\forall \sigma \in S:\|\sigma\|_{r} \geq c\|\sigma\|_{S} \eta(r) \quad(r \in \rho)
$$

Beweis. Nach Voraussetzung ist $\|\sigma\|_{r} \underset{\rho}{\sim} \eta$ für alle betrachteten $\sigma$; es gibt also Konstanten $c(\sigma)$, so daß

$$
\|\sigma\|_{r} \geq c(\sigma) \eta(r) \quad(r \in \rho)
$$


Wir zeigen, daß diese Konstanten lokal gleichmäßig gewählt werden können. Dazu sei $\left\{\sigma_{1}, \ldots, \sigma_{n}\right\}$ eine Basis von $S$. Für $\epsilon \in \mathbb{R}^{n}$ und festes $\sigma \in S$ gilt

$$
\left\|\sigma+\sum_{i} \epsilon_{i} \sigma_{i}\right\|_{r} \geq\|\sigma\|_{r}-\sum_{i}\left|\epsilon_{i}\right|\left\|\sigma_{i}\right\|_{r} .
$$

Da $\left\|\sigma_{i}\right\| \underset{\rho}{\sim} \eta$, können wir Konstanten $d_{i}>0$ wählen, so daß $\left\|\sigma_{i}\right\|_{r} \leq d_{i} \eta(r)$ für $r \in \rho$; folglich wird

$$
\left\|\sigma+\sum_{i} \epsilon_{i} \sigma_{i}\right\|_{r} \geq c(\sigma) \eta(r)-\sum_{i}\left|\epsilon_{i}\right| d_{i} \eta(r)=\underbrace{\left(c(\sigma)-\sum_{i}\left|\epsilon_{i}\right| d_{i}\right)}_{\geq \frac{1}{2} c(\sigma) \text { für kleine } \epsilon} \eta(r),
$$

d.h. wir können die Konstanten $c(\sigma)$ lokal gleichmäßig wählen. Da die Einheitskugel bzgl. $\|\cdot\|_{S}$ kompakt ist,] können wir die Konstante dann auf der gesamten Einheitskugel gleichmäßig wählen; es gibt also $c>0$, so daß

$$
\|\sigma\|_{r} \geq c\|\sigma\|_{S} \eta(r) \quad \forall r \in \rho,
$$

was zu zeigen war.

Wir wollen nun allgemeinere endlichdimensionale Räume $S \subset \overline{\mathfrak{A}}_{*}$ in direkte Summen aus homogenen Räumen (wie oben beschrieben) zerlegen. Dies wird im allgemeinen nur möglich sein, indem man, ausgehend von einer gegebenen Nullfolge $\rho$, zu Unterfolgen übergeht.

Dazu zunächst eine Vorbemerkung: Ist

$$
S=S_{1} \oplus S_{2} \quad\left(S, S_{1}, S_{2} \subset \overline{\mathfrak{A}}_{*}\right),
$$

so haben wir für festes $r$ folgende Situation, wobei wir mit $i_{S}$ die Inklusion $S \hookrightarrow \mathfrak{A}(r)_{*}$ usw. bezeichnen:

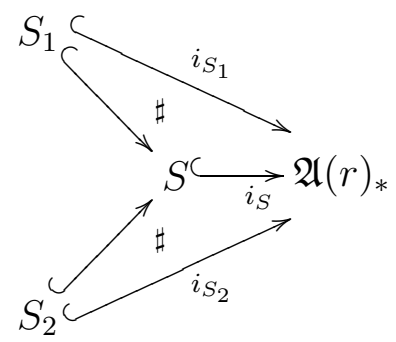

Im folgenden wollen wir die Normen der hier eingezeichneten Inklusionsabbildungen analysieren, und zwar ist

$$
\left\|i_{S}\right\|_{r}=\sup _{\sigma \in S} \frac{\left\|i_{S} \sigma\right\|_{r}}{\|\sigma\|_{S}}
$$

mit einer fest gewählten Norm $\|\cdot\|_{S}$ auf $S$. (Es ist für das später zu betrachtende asymptotische Verhalten gleichgültig, welche Norm auf $S$ gewählt wird: Da für eine andere Norm

\footnotetext{
${ }^{7}$ Jede Norm auf dem endlichdimensionalen Raum $S$ induziert die Standardtopologie.
} 
$\|\cdot\|_{S}^{\prime}$ stets Konstanten $a, b>0$ existieren, so daß $a\|\sigma\|_{S} \leq\|\sigma\|_{S}^{\prime} \leq b\|\sigma\|_{S}$, wird dann $\left\|i_{S}\right\|_{r} \sim\left\|i_{S}\right\|_{r}^{\prime}$, d.h. asymptotisch ergibt sich kein Unterschied.)

Für die Abbildungen $i_{S_{1}}, i_{S_{2}}$ haben wir zunächst zwei Normbegriffe: Einerseits ist analog $\mathrm{zu}(3 . \mathrm{A} .7)$

$$
\left\|i_{S_{j}}\right\|_{r}=\sup _{\sigma \in S_{j}} \frac{\left\|i_{S_{j}} \sigma\right\|_{r}}{\|\sigma\|_{S_{j}}}
$$

andererseits kann man die Norm der Inklusionen als Abbildungen auf $S$ (fortgesetzt durch 0 auf dem Komplement) betrachten:

$$
\left\|i_{S_{1}} \oplus 0\right\|_{r}=\sup _{\sigma \in S} \frac{\left\|\left(i_{S_{1}} \oplus 0\right) \sigma\right\|_{r}}{\|\sigma\|_{S}}=\sup _{\sigma_{j} \in S_{j}} \frac{\left\|\sigma_{1}\right\|_{r}}{\left\|\sigma_{1}+\sigma_{2}\right\|_{S}} .
$$

Diese beiden Begriffe sind jedoch für unsere Zwecke gleichwertig: Wir wählen dazu die Norm auf $S$ zunächst als

$$
\left\|\sigma_{1} \oplus \sigma_{2}\right\|_{S}:=\left\|\sigma_{1}\right\|_{S_{1}}+\left\|\sigma_{2}\right\|_{S_{2}}
$$

(das definiert tatsächlich eine Norm). Dann ist offenbar

$$
\left\|i_{S_{1}} \oplus 0\right\|_{r}=\sup _{\sigma_{1} \in S_{1}, \sigma_{2} \in S_{2}} \frac{\left\|\sigma_{1}\right\|_{r}}{\left\|\sigma_{1}\right\|_{S_{1}}+\left\|\sigma_{2}\right\|_{S_{2}}}=\sup _{\sigma_{1} \in S_{1}} \frac{\left\|\sigma_{1}\right\|_{r}}{\left\|\sigma_{1}\right\|_{S_{1}}}=\left\|i_{S_{1}}\right\|_{r} .
$$

Bei anderer Wahl der Normen auf $S, S_{j}$ gilt immerhin noch

$$
\left\|i_{S_{1}} \oplus 0\right\|_{r} \underset{\text { as }}{\sim}\left\|i_{S_{1}}\right\|_{r}
$$

wegen der Äquivalenz aller Normen auf endlichdimensionalen Räumen. Interessieren wir uns also nur für das asymptotische Verhalten, dann müssen wir die beiden Normbegriffe nicht unterscheiden, wovon wir im folgenden öfters Gebrauch machen.

Es seien nun ein endlichdimensionaler (mindestens eindimensionaler) Raum $S \subset \overline{\mathfrak{A}}_{*}$ und eine Nullfolge $\rho$ vorgegeben; wir wollen $S$ bezüglich einer geeignete Teilfolge von $\rho$ in eine direkte Summe homogener Teilräume zerlegen.

Dazu wählen wir zunächst eine Teilfolge $\rho^{\prime} \subset \rho$ und Unterräume $S_{1}, S_{2} \subset S$ derart, daß

$$
\begin{gathered}
S=S_{1} \oplus S_{2}, \\
\left\|i_{S_{1}}\right\|_{r} \underset{\rho^{\prime}}{\gg}\left\|i_{S_{2}}\right\|_{r}
\end{gathered}
$$

und so, daß $\operatorname{dim} S_{1}$ unter diesen Nebenbedingungen minimal wird. (Mit $S_{1}=S, S_{2}=\{0\}$ läßt sich sicherlich eine Zerlegung finden, die (3.A.13) und (3.A.14) erfüllt, so daß wir das Minimum nicht über die leere Menge bilden.) Durch diese Wahl, so behaupten wir, wird $S_{1}$ homogen.

Dies begründet sich folgendermaßen: Sicher ist $\operatorname{dim} S_{1}>0$ (wegen (3.A.14), solange $\operatorname{dim} S>0$ ). Für $\operatorname{dim} S_{1}=1$ ist $S_{1}$ trivialerweise homogen. Sei also $\operatorname{dim} S_{1}>1$. Wir nehmen an, daß es $\sigma \in S_{1}$ gibt, so daß

$$
\|\sigma\|_{r} \underset{\rho^{\prime}}{\not}\left\|i_{S_{1}}\right\|_{r}
$$


Sicher gilt aber $\|\sigma\|_{r} \leq\left\|i_{\rho_{1}}\right\|_{r}$, so daß bezüglich einer gewissen Teilfolge $\rho^{\prime \prime} \subset \rho^{\prime}$ gelten muß

$$
\|\sigma\|_{r} \underset{\rho^{\prime \prime}}{\ll}\left\|i_{S_{1}}\right\|_{r}
$$

Wir zerlegen dann $S_{1}=S_{1}^{\prime} \oplus \mathbb{C} \sigma$ mit geeignetem $S_{1}^{\prime} \subset S_{1}$ und zeigen, daß

$$
S=S_{1}^{\prime} \oplus \underbrace{\left(\mathbb{C} \sigma \oplus S_{2}\right)}_{S_{2}^{\prime}}
$$

ebenfalls eine Zerlegung von $S$ ist, die (3.A.14) erfüllt: Es ist nämlich

$$
\left\|i_{S_{1}^{\prime}}\right\|_{r} \underset{\rho^{\prime \prime}}{\sim}\left\|i_{S_{1}^{\prime}} \oplus 0\right\|_{r}=\left\|i_{S_{1}}-0 \oplus i_{\mathbb{C} \sigma}\right\|_{r} \geq\left\|i_{S_{1}}\right\|_{r}-\underbrace{\left\|0 \oplus i_{\mathbb{C} \sigma}\right\|_{r}}_{\substack{\ll \prime \rho^{\prime \prime} \\ \|} i_{S_{1}} \|_{r}} \underset{\rho^{\prime \prime}}{\sim}\left\|i_{S_{1}}\right\|_{r}
$$

und

$$
\left\|i_{S_{2}^{\prime}}\right\| \underset{\rho^{\prime \prime}}{\leq}\left\|0 \oplus i_{S_{2}}\right\|_{r}+\left\|i_{\mathbb{C} \sigma}\right\|_{r} \underset{\rho^{\prime \prime}}{\ll}\left\|i_{S_{1}}\right\|_{r}
$$

also

$$
\left\|i_{S_{1}^{\prime}}\right\|_{r} \underset{\rho^{\prime \prime}}{\gg}\left\|i_{S_{2}^{\prime}}\right\|_{r}
$$

Damit ist ein Widerspruch zur geforderten Minimalität von $\operatorname{dim} S_{1}$ erreicht, und es gilt doch $\|\sigma\|_{r} \underset{\rho^{\prime}}{\sim}\left\|i_{S}\right\|_{r}$. Folglich ist $S_{1} \underset{\rho^{\prime}}{\sim}\left\|i_{S}\right\|_{r}=: \eta(r)$ homogen. Wir erhalten also folgende Aussage:

Satz 3.22. Sei $S \subset \overline{\mathfrak{A}}_{*}$ ein endlichdimensionaler Teilraum, $S \neq\{0\}$, und $\rho$ eine Nullfolge. Dann gibt es eine Teilfolge $\rho^{\prime} \subset \rho$, einen bezüglich $\rho^{\prime}$ homogenen Teilraum $S_{1} \subset S$ und einen weiteren Teilraum $S_{2} \subset S$, so daß

$$
S=S_{1} \oplus S_{2}, \quad\left\|i_{S_{1}}\right\|_{r} \underset{\rho^{\prime}}{\gg}\left\|i_{S_{2}}\right\|_{r}
$$

Wenden wir diesen Satz wiederum auf $S_{2}$ an (es gilt $\operatorname{dim} S_{2}<\operatorname{dim} S$ ) und iterieren dieses Verfahren, bis als zweiter Summand nur noch der Nullraum übrig bleibt, dann erhalten wir:

Satz 3.23. Sei $S \subset \overline{\mathfrak{A}}_{*}$ ein endlichdimensionaler Teilraum, $S \neq\{0\}$, und $\rho$ eine Nullfolge. Dann gibt es eine Teilfolge $\rho^{\prime} \subset \rho$ und bezüglich $\rho^{\prime}$ homogene Teilräume $S_{1}, \ldots, S_{n} \subset S$, so $d a \beta$

$$
S=S_{1} \oplus \ldots \oplus S_{n}, \quad\left\|i_{S_{1}}\right\|_{r} \underset{\rho^{\prime}}{\gg} \ldots \underset{\rho^{\prime}}{\gg}\left\|i_{S_{n}}\right\|_{r}
$$




\section{B Eine Erweiterung des Satzes von Hahn-Banach}

Der Satz von Hahn-Banach wird üblicherweise für die Fortsetzung linearer Funktionale auf einem Banachraum formuliert. Wir benötigen eine leicht erweiterte Fassung, die nicht lineare Abbildungen nach $\mathbb{C}$, sondern solche mit einem allgemeinen endlichdimensionalen Vektorraum $V$ als Bildraum behandelt. Der Satz läßt sich auf diese Situation leicht verallgemeinern:

Satz 3.24. Es sei $V$ ein endlichdimensionaler $\mathbb{C}$-Vektorraum mit Norm $\|\cdot\|_{V}$. Dann gibt es eine Konstante $c>0$ mit der folgenden Eigenschaft: Ist $\left(B,\|\cdot\|_{B}\right)$ ein Banachraum und $U \subset B$ ein abgeschlossener Unterraum, dann läßt sich jeder lineare stetige Operator $A: U \rightarrow V$ zu einem linearen stetigen Operator $\hat{A}: B \rightarrow V$ fortsetzen (d.h. $\hat{A}\lceil U=A)$, so $d a \beta$

$$
\|\hat{A}\| \leq c \cdot\|A\|
$$

(Die Konstante c hängt hier von der Wahl der Norm $\|\cdot\|_{V}$, nicht aber von $A$ oder $\|\cdot\|_{B}$ ab.)

Beweis. Wir wählen eine Basis $\left\{v_{i}\right\}_{i=1}^{n}$ von $V$ aus Einheitsvektoren und bezeichnen mit $p_{i}$ die Projektionen auf die zugehörigen Komponenten. Dann sind die Funktionale $\varphi_{i}=p_{i} \circ A$ stetige lineare Funktionale auf $U$; sie lassen sich nach dem Satz von Hahn-Banach also zu Funktionalen $\hat{\varphi}_{i}$ auf $B$ fortsetzen, ohne ihre Norm zu vergrößern. Wir setzen nun

$$
\hat{A}=\sum_{i=1}^{n} \hat{\varphi}_{i}(\cdot) v_{i}
$$

Dann gilt

$$
\hat{A}\left\lceil U=\sum_{i=1}^{n}\left(\hat{\varphi}_{i}(\cdot)\lceil U) v_{i}=\sum_{i=1}^{n}\left(p_{i}(\cdot) \circ A\right) v_{i}=A,\right.\right.
$$

und wir erhalten die Normabschätzung

$$
\|\hat{A}\| \leq \sum_{i=1}^{n}\left\|\hat{\varphi}_{i}(\cdot)\right\| \underbrace{\left\|v_{i}\right\|}_{=1}=\sum_{i=1}^{n}\left\|\varphi_{i}(\cdot)\right\| \leq \underbrace{\sum_{i=1}^{n}\left\|p_{i}(\cdot)\right\|}_{=: c}\|A\|=c\|A\|,
$$

wobei $c$ nur von der Wahl der Einheitsvektoren $v_{i}$ (und damit von $\|\cdot\|_{V}$ ) abhängt.

\section{C Linearformen mit polynomialen Energieschranken}

Für die Konstruktion punktartig lokalisierter Felder $\phi$ ist es wichtig, die scharfe Energieabschneidung $P(E) \phi P(E)$ abzuschwächen und etwa zu der von Fredenhagen und Hertel [FH81] verwendeten Energiedämpfung $R^{l} \phi R^{l}$ überzugehen. Dabei ist $R$ der wegen der Spektrumsbedingung beschränkte Operator $R=(1+H)^{-1}$. Als wesentliches Bindeglied zwischen den beiden Formen von Energieschranken erweist sich folgende Formel: 
Lemma 3.25. Im Sinne schwacher Konvergenz auf $\mathcal{H} \times \mathcal{H}$ gilt für $l \in \mathbb{N}$ :

$$
R^{l}=l \int_{0}^{\infty} d E\left(\frac{1}{1+E}\right)^{l+1} P(E)
$$

Beweis. Es seien $f(E)$ eine stetig differenzierbare, $g(E)$ eine monoton wachsende und beschränkte Funktion auf $\mathbb{R}$ derart, daß die im folgenden auftretenden Integrale existieren. $g$ ist dann bis auf abzählbar viele „Sprungstellen“ $E_{i}$ differenzierbar mit Ableitung $g^{\prime}$. Man wertet folgendes Riemann-Stieltjes-Integral aus:

$$
\int f(E) d g(E)=\int f(E) g^{\prime}(E) d E+\sum_{i} f\left(E_{i}\right)\left(g\left(E_{i}+\right)-g\left(E_{i}-\right)\right) .
$$

Außerdem berechnet man durch partielle Integration:

$$
\int-f^{\prime}(E) g(E) d E=\sum_{i}\left(\int_{E_{i}}^{E_{i+1}} f(E) g^{\prime}(E) d E-f\left(E_{i+1}\right) g\left(E_{i+1}-\right)+f\left(E_{i}\right) g\left(E_{i}+\right)\right) \text {. }
$$

(Dabei sind eventuelle Randterme bei $E= \pm \infty$ in suggestiver Weise notiert.) Die beiden Integrale (3.C.1) und (3.C.2) stimmen also überein. Setzt man nun

$$
f(E)=\left(\frac{1}{1+E}\right)^{l}, \quad g(E)=(\psi \mid P(E) \psi) \quad(\psi \in \mathcal{H})
$$

dann folgt die Behauptung zumindest auf Zuständen $(\psi \mid \cdot \psi)$. Die Polarisationsidentität liefert die Erweiterung auf alle Matrixelemente.

Wir können hieraus eine etwas technische, aber sehr nützliche Abschätzung folgern:

Lemma 3.26. $Z u l \in \mathbb{N}$ gibt es eine Konstante $d>0$ mit folgender Eigenschaft: Sei $B \in \mathfrak{B}(\mathcal{H})$, und sei $c>0$, so daß

$$
\|P(E) B P(E)\| \leq c \cdot E^{l-1} \quad \forall E \geq 1 .
$$

Dann folgt

$$
\left\|R^{l} B R^{l}\right\| \leq c \cdot d
$$

Ein wichtiger Aspekt ist hierbei, daß die Konstante $d$ universell gewählt werden kann und nicht von $B$ oder $c$ abhängt.

Beweis. Wir können aus der Abschätzung für $\|P(E) B P(E)\|$ folgern, daß für beliebige $E_{1}, E_{2}>0$

$$
\left\|P\left(E_{1}\right) B P\left(E_{2}\right)\right\| \leq\left(1+\max \left\{E_{1}, E_{2}\right\}\right)^{l-1} \cdot c .
$$


Nun berechnet man für Einheitsvektoren $\xi, \xi^{\prime} \in \mathcal{H}$ :

$$
\begin{aligned}
& \left|\left(\xi \mid R^{l} B R^{l} \xi^{\prime}\right)\right| \\
& \qquad \underset{(\text { Lemma } 3.25)}{=}\left|l^{2} \int_{0}^{\infty} d E_{1} \int_{0}^{\infty} d E_{2}\left(\frac{1}{1+E_{1}} \cdot \frac{1}{1+E_{2}}\right)^{l+1}\left(\xi\left|P\left(E_{1}\right) B P\left(E_{2}\right)\right| \xi^{\prime}\right)\right| \\
& \quad \leq c \cdot l^{2} \int_{0}^{\infty} d E_{1} \int_{0}^{\infty} d E_{2}\left(\frac{1}{1+E_{1}} \cdot \frac{1}{1+E_{2}}\right)^{l+1}\left(1+\max \left\{E_{1}, E_{2}\right\}\right)^{l-1}
\end{aligned}
$$

Dieses Integral existiert, da der Integrand mindestens wie $E_{i}^{-2}$ abfällt. Damit ist eine von $\xi, \xi^{\prime}, B$ und $c$ unabhängige Abschätzung gefunden.

Wir können dieses Lemma auch auf unbeschränkte Linearformen ausdehnen, solange deren Hochenergieverhalten polynomial ist:

Lemma 3.27. Sei $\phi \in \bar{\Sigma}^{*}$ eine Linearform mit

$$
\|\phi\|_{E} \leq E^{l-1} \cdot c \quad \text { für ein } l \in \mathbb{N} \text {, ein } c>0 \text { und alle } E \geq 1 \text {. }
$$

Dann läßt sich $R^{l} \phi R^{l} z u$ einem beschränkten Operator erweitern, und es gilt

$$
\left\|R^{l} \phi R^{l}\right\| \leq c \cdot d
$$

mit einer von $\phi$ und c unabhängigen Konstanten $d$.

Beweis. Sei zunächst $E^{\prime} \geq 1$ fest. Wir setzen $B=P\left(E^{\prime}\right) \phi P\left(E^{\prime}\right)$. Nach Voraussetzung ist

$$
\|P(E) B P(E)\| \leq E^{l-1} \cdot c .
$$

Lemma 3.26 liefert dann

$$
\left\|P\left(E^{\prime}\right) R^{l} \phi R^{l} P\left(E^{\prime}\right)\right\|=\left\|R^{l} B R^{l}\right\| \leq c \cdot d
$$

Da diese Abschätzung unabhängig von $E^{\prime}$ ist, läßt sich $R^{l} \phi R^{l}$ zu einem beschränkten Operator fortsetzen.

Wir interessieren uns außerdem für die Vertauschungsrelationen von $R$ mit den durch Testfunktionen $f$,verschmierten“ quadratischen Formen

$$
\phi(f)=\int d^{s+1} x f(x) U(x) \phi U(x)^{*}, \quad f \in \mathcal{S}(\mathcal{M}) .
$$

Dabei erfülle $\phi$ die Bedingungen aus Lemma 3.27; das Integral (3.C.8) ist dann im Sinne von Linearformen auf $\bar{\Sigma}$ erklärt. Da $f$ absolut integrierbar ist, gilt

$$
\|\phi(f)\|_{E} \leq \int d^{s+1} x|f(x)|\|\phi\|_{E}<E^{l-1} \cdot \text { const. }
$$


also existiert auch $R^{l} \phi(f) R^{l}$ als beschränkter Operator. Wir bemerken, daß für das energiegedämpfte Feld die Integralformel (3.C.8) sogar stärker gilt: Man hat

$$
R^{l+1} \phi(f) R^{l+1}=\int d^{s+1} x f(x) U(x) R^{l+1} \phi R^{l+1} U(x)^{*}
$$

im Sinne von Normkonvergenz, d.h. als Bochner-Integral. (Das sieht man so: Das Integral auf der rechten Seite existiert sicherlich, da $U(x) R$ normstetig ist, wie (3.4.12) zeigt. Auf Funktionalen $\sigma \in \bar{\Sigma}$ stimmen die linke und die rechte Seite wegen (3.C.8) überein, also sind sie auch als Operatoren gleich.) Wir zeigen nun:

Lemma 3.28. Sei $f \in \mathcal{S}(\mathcal{M})$; dann gilt im Sinne von Operatoren auf $\mathcal{C}^{\infty}(\mathcal{H})$ :

$$
[R, \phi(f)]=-i R \phi\left(\partial_{t} f\right) R .
$$

Beweis. Sei im folgenden $E \geq 1$ fest, und sei $\sigma \in \Sigma(E)$. Da $P(E) R^{-1}$ beschränkt ist, hat man

$$
\sigma([R, \phi(f)])=\sigma\left(\frac{1}{1+H}[\phi(f),(1+H)] \frac{1}{1+H}\right)=-\sigma(R[H, \phi(f)] R) .
$$

Wir setzen $\sigma^{\prime}:=\sigma(R \cdot R)$; auch dieses Funktional liegt in $\Sigma(E)$. Da die Operatoren $P(E) P_{\mu}$ beschränkt sind, können im Ausdruck

$$
\sigma^{\prime}\left(U(x) \phi U(x)^{*}\right)
$$

die Translationsoperatoren $U(x)=e^{i P_{\mu} x^{\mu}}$ als Potenzreihe ausgeschrieben werden, und (3.C.13) wird damit eine differenzierbare Funktion von $x$. Man berechnet nun:

$$
\begin{aligned}
& \partial_{t} \sigma^{\prime}\left(U(x) \phi U(x)^{*}\right)=\frac{\partial}{\partial x^{0}} \sigma^{\prime}\left(e^{i P_{\mu} x^{\mu}} \phi e^{-i P_{\mu} x^{\mu}}\right) \\
& =i \sigma^{\prime}\left(P_{0} U(x) \phi U(x)^{*}-U(x) \phi U(x)^{*} P_{0}\right)=i \sigma^{\prime}\left(\left[H, U(x) \phi U(x)^{*}\right]\right) .
\end{aligned}
$$

Damit ergibt sich schließlich

$$
\begin{array}{rl}
\sigma^{\prime}([H, \phi(f)])=\int d^{s+1} x & f(x) \sigma^{\prime}\left(\left[H, U(x) \phi U(x)^{*}\right]\right) \\
= & -i \int d^{s+1} x f(x) \partial_{t} \sigma^{\prime}\left(U(x) \phi U(x)^{*}\right) \\
& \stackrel{\text { (P.I.) }}{=} i \int d^{s+1} x \partial_{t} f(x) \sigma^{\prime}\left(U(x) \phi U(x)^{*}\right)=i \sigma^{\prime}\left(\phi\left(\partial_{t} f\right)\right) .
\end{array}
$$

Zusammen mit (3.C.12) liefert das die Behauptung zunächst im Sinne von Linearformen auf $\bar{\Sigma}$, was sich dann als Operatorgleichung fortsetzen läßt.

Für den Beweis des Lemmas mußte hinsichtlich der Funktion $f$ nur ausgenutzt werden, daß in (3.C.15) partiell integriert werden kann und daß $\partial_{t} f$ absolut integrabel ist. Wir müssen $f$ also nicht als Schwartzfunktion annehmen; es reicht, wenn $f$ eine verallgemeinerte Ableitung im Sinne von Sobolevräumen besitzt. In der Anwendung (Satz 3.13) bedeutet dies, daß man - bei oben festgehaltenem $l$ - die $\phi(f)$ zu unbeschränkten Operatoren erweitern kann, sobald $f$ verallgemeinerte Ableitungen $l$-ter Ordnung besitzt. Bei festem $l$ kann man die Klasse der zulässigen Testfunktionen für die operatorwertige Distribution $\phi$ also über $\mathcal{S}(\mathcal{M})$ hinaus erweitern. (Vgl. hierzu auch [BDH95, §8].) 


\section{Kapitel 4}

\section{Symmetrien}

Ein wichtiger Aspekt bei der Klassifikation und Analyse von Quantenfeldtheorien sind die Darstellungen von Symmetriegruppen, wie der Poincaré-Gruppe, der Dilatationsgruppe oder innerer Symmetrien der Theorie. Wir untersuchen in diesem Kapitel das Verhalten der konstruierten Punktfelder unter solchen Symmetrietransformationen.

Dazu geben wir zunächst eine vereinfachte Version des betrachteten Punktfeld-Formalismus an, der zwar nicht alle Aspekte des Phasenraumkriteriums enthält, sich für die Betrachtung von Symmetrien aber als geeignet erweist.

In diesem Rahmen untersuchen wir dann allgemein die Übertragung von Kovarianzeigenschaften der algebraischen Theorie auf die konstruierten Räume von Punktfeldern. Von den betrachteten Transformationen müssen wir voraussetzen, daß sie Lokalisierungseigenschaften in Orts- und Impulsraum nicht zu stark stören; in diesem Fall kann gezeigt werden, daß sie die Punktfeld-Vektorräume in sich überführen, daß sich die Felder also kovariant unter der Transformation verhalten.

Als konkrete Anwendung behandeln wir die Lorentzkovarianz der Punktfelder, deren Hermitezität sowie eine mögliche Kovarianz unter Dilatationen (als Zusatzstruktur).

Bis hierher wurden stets am Koordinatenursprung lokalisierte Punktfelder betrachtet; alle Strukturen wurden für diesen Spezialfall definiert. Das stellt wegen der Translationssymmetrie der Theorie keine Einschränkung dar. Diese Tatsache wird präzisiert: Wir geben explizit eine Wirkung der Translationen auf den Punktfeldern an und etablieren so die Kovarianz der Punktfelder bzw. Wightman-Distributionen unter der vollen Poincaré-Gruppe. Insbesondere sind damit die Wightman-Axiome für die konstruierten Punktfelder etabliert.

Außerdem untersuchen wir partielle Ableitungen der Punktfelder, die in der Wightman'schen Feldtheorie zur Formulierung von Feldgleichungen wichtig sind. Wir zeigen, daß die Menge der konstruierten Felder abgeschlossen unter Differentiation ist. 


\subsection{Reformulierung der Struktur}

Wir haben bisher unsere Strukturen in der Sprache bilinearer Abbildungen $\bar{\Sigma} \times \overline{\mathfrak{A}} \rightarrow \mathbb{C}$ formuliert und ihr Verhalten im Limes kleiner Wirkungen $E \cdot r \rightarrow 0$ betrachtet. Das asymptotische Phasenraumkriterium mit polynomialen Energieschranken, das wir in diesem und in folgenden Kapiteln stets als gegeben annehmen, erlaubt uns die Analyse des Punktfeldinhalts. Dabei war die gleichzeitige Betrachtung von $E$ - und $r$-Verhalten unbedingt notwendig, um die Lokalitätseigenschaften der Punktfelder zu etablieren, wie wir in Kapitel 3 gesehen hatten.

Jetzt, da wir diese Analyse durchgeführt haben, können wir den Formalismus etwas vereinfachen und das $E$ - vom $r$-Verhalten trennen. Dies führt uns zu einer Formulierung, die die Räume $\Phi_{\bar{\gamma}}$ statt der bilinearen Abbildungen als zentrales Element in den Mittelpunkt stellt, und die näher an den von Haag und Ojima [H(96] ursprünglich vorgeschlagenen Strukturen liegt.

Wir halten dazu im folgenden $E$ zunächst fest. Ist $E$ dabei hinreichend groß, dann können wir $\Phi_{\bar{\gamma}}$ als Teilraum von $\Sigma(E)^{*}$ betrachten; die Inklusionsabbildung nennen wir $j_{\bar{\gamma}}$ :

$$
\Sigma(E)^{*} \underset{j_{\bar{\gamma}}}{\longleftarrow} \Phi_{\bar{\gamma}}
$$

Diese Inklusion ist trivialerweise stetig (da auf $\Phi_{\bar{\gamma}}$ die Teilraumtopologie mit der Standardtopologie übereinstimmt). Betrachten wir also den (Prä-)Dualraum des endlichdimensionalen Raums $\Phi_{\bar{\gamma}}\left(\Phi_{\bar{\gamma} *}=: \Sigma_{\bar{\gamma}}\right)$, dann kann man die Abbildung $j_{\bar{\gamma}}$ als ,präduale“ Abbildung $j_{\bar{\gamma} *}$ auf die Prädualräume übertragen:

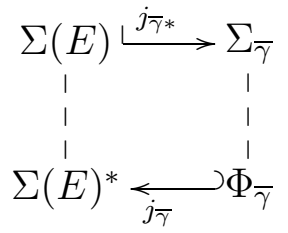

Bei genügend großem $E$ ist $j_{\bar{\gamma} *}$ dabei surjektiv, d.h. wir können $j_{\bar{\gamma} *}$ als Projektionsabbildung bezüglich einer Quotientenbildung verstehen. Diese wollen wir genauer untersuchen; es stellt sich also die Frage nach einer Charakterisierung von Kern $j_{\bar{\gamma} *}$. Die Antwort darauf gibt folgendes Lemma:

Lemma 4.1. Im Diagramm (4.1.2) gilt:

$$
\operatorname{Kern} j_{\bar{\gamma} *}=\left\{\sigma \in \Sigma(E) \mid \gamma\left(\Xi_{\mathrm{L}} \sigma\right) \geq \bar{\gamma}\right\}
$$

Beweis. Man hat per Definition

$$
\sigma \in \operatorname{Kern} j_{\bar{\gamma} *} \quad \Leftrightarrow \quad\left(j_{\bar{\gamma} *} \sigma\right)(\phi)=0 \quad \forall \phi \in \Phi_{\bar{\gamma}} \quad \Leftrightarrow \quad \sigma\left(j_{\bar{\gamma}} \phi\right)=0 \quad \forall \phi \in \Phi_{\bar{\gamma}},
$$

d.h. $\sigma$ liegt im Kern von $j_{\bar{\gamma} *}$ genau dann, wenn $\sigma$ auf dem Teilraum $\Phi_{\bar{\gamma}} \subset \bar{\Sigma}$ verschwindet. Nach Satz 3.2 ist das äquivalent zu

$$
\gamma\left(\Xi_{\mathrm{L}} \sigma\right) \geq \bar{\gamma}
$$

womit das Lemma bewiesen ist. 
Wir können damit schreiben:

$$
\Sigma_{\bar{\gamma}}=\Sigma(E) /\left\{\sigma \in \Sigma(E) \mid \gamma\left(\Xi_{\mathrm{L}} \sigma\right) \geq \bar{\gamma}\right\} .
$$

Der Raum $\Sigma_{\bar{\gamma}}$ ist also quasi der Raum aller energiebeschränkten Funktionale, wobei diejenigen identifiziert werden, deren Differenz im Limes $r \rightarrow 0$ auf $\mathfrak{A}(r)$ schnell verschwindet - man beachte hierzu, daß nach (2.D.7) gilt

$$
\gamma\left(\Xi_{\mathrm{L}} \sigma\right) \geq \bar{\gamma} \quad \Leftrightarrow \quad r^{-\bar{\gamma}+\epsilon}\|\sigma\|_{r} \stackrel{r \rightarrow 0}{\longrightarrow} 0 \quad \forall \epsilon>0 .
$$

Damit entsprechen die $\Sigma_{\bar{\gamma}}$ den Halmen der Prägarbe $\Sigma(E)\lceil\mathfrak{A}(r)$ bezüglich der betrachteten Äquivalenzrelation; ihre Elemente sind gerade die von Haag und Ojima in [H(0)6] betrachteten Zustandskeime („germs of states“). Die Dualräume $\Phi_{\bar{\gamma}}=\Sigma_{\bar{\gamma}}^{*}$ bilden den Punktfeldinhalt der Theorie (zu einem gegebenen Kurzabstandsverhalten $r^{\bar{\gamma}}$ ). Wir werden die $\Sigma_{\bar{\gamma}}$ daher auch als Zustandshalme und die $\Phi_{\bar{\gamma}}$ als Feldhalme bezeichnen.

Wir skizzieren noch die Situation bei Variation von $\bar{\gamma}$ und $E$. Für verschiedene $E, E^{\prime}$ (etwa $E<E^{\prime}$ ) erhält man, wie Lemma 4.1 zeigt, das folgende Diagramm von zueinander dualen Räumen und Abbildungen:

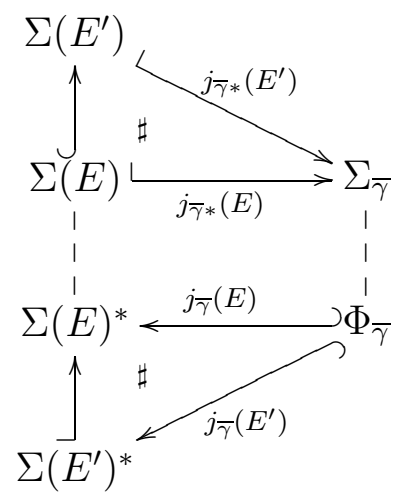

Das ist hier nur der Vollständigkeit halber erwähnt - die Abbildungen $j_{\bar{\gamma}}, j_{\bar{\gamma} *}$ kann man in

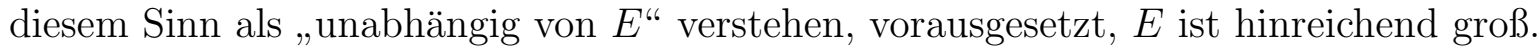

Bei Variation von $\bar{\gamma}$ - wir geben uns zwei Werte $\bar{\gamma}^{\prime}<\bar{\gamma}$ vor - kennen wir bereits aus früheren Kapiteln eine natürliche Inklusion $j_{\bar{\gamma}^{\prime} \bar{\gamma}}: \Phi_{\bar{\gamma}^{\prime}} \hookrightarrow \Phi_{\bar{\gamma}}$, die uns folgendes Diagramm liefert:

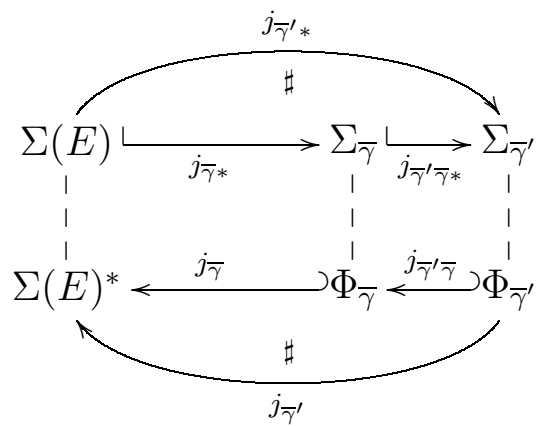

Wie von Haag und Ojima erwartet, erhalten wir also eine ganze „Kaskade“ der Räume von Zustandskeimen

$$
\ldots \Sigma_{n} \longrightarrow \Sigma_{n-1} \longleftrightarrow \Sigma_{n-2} \longrightarrow \cdots
$$


und eine entsprechende Kette für die Räume von Punktfeldern auf der dualen Seite:

$$
\ldots \Phi_{n-2} \longleftrightarrow \Phi_{n-1} \longleftrightarrow \Phi_{n} \longleftrightarrow \cdots
$$

Die Indizierung mit $n, n-1$ etc. soll dabei darauf hinweisen, daß man gewisse geordnete Werte für $\bar{\gamma}$ auswählt; die Schritte müssen nicht notwendigerweise ganzzahlig sein. Sinnvoll ist sicher, sie so zu wählen, daß alle in der Kette auftretenden Räume unterschiedliche Dimension haben, so daß die Inklusionen bzw. Projektionen „echt“ sind.

Wir wollen nun diese „Kette“ von Vektorräumen in Abhängigkeit von $\bar{\gamma}$ genauer untersuchen. Dazu betrachten wir die Funktion

$$
d(\bar{\gamma})=\operatorname{dim} \Phi_{\bar{\gamma}}: \mathbb{R}^{+} \rightarrow \mathbb{N}_{0} .
$$

Sie ist monoton wachsend und deshalb stückweise konstant; zwischen den konstanten Stücken liegen diskrete Unstetigkeitsstellen. Es sei $\bar{\gamma}_{0}$ eine solche Stelle, d.h. es gelte in einer Umgebung von $\bar{\gamma}_{0}$ :

$$
d(\bar{\gamma})= \begin{cases}n_{1} & \text { für } \bar{\gamma}<\bar{\gamma}_{0}, \\ n_{2} & \text { für } \bar{\gamma}=\bar{\gamma}_{0}, \\ n_{3} & \text { für } \bar{\gamma}>\bar{\gamma}_{0} .\end{cases}
$$

Dabei ist $n_{1} \leq n_{2} \leq n_{3}$. Um die Stetigkeitseigenschaften bei $\bar{\gamma}_{0}$ zu untersuchen, nehmen wir $n_{1}<n_{2}$ an; dann ist also $\Phi_{\left(\bar{\gamma}_{0}-\epsilon\right)} \subsetneq \Phi_{\bar{\gamma}_{0}}$ für kleine $\epsilon>0$; entsprechende echte Inklusionen gelten für die $\Sigma_{\bar{\gamma}}$ und Kern $j_{\bar{\gamma} *}$. Man beachte, daß auf den Intervallen, auf denen $d(\bar{\gamma})$ konstant ist, sich auch die Räume $\Phi_{\bar{\gamma}}, \Sigma_{\bar{\gamma}}$, Kern $j_{\bar{\gamma} *}$ selbst nicht ändern. Wir können also in unserer Situation ein $\sigma \in \bar{\Sigma}$ wählen, so daß

$$
\sigma \notin \operatorname{Kern} j_{\bar{\gamma}_{0} *}, \quad \sigma \in \operatorname{Kern} j_{\left(\bar{\gamma}_{0}-\epsilon\right) *} \quad \text { für kleine } \epsilon>0 .
$$

Nach Lemma 4.1$]$ bedeutet dies

$$
\gamma\left(\Xi_{\mathrm{L}} \sigma\right)<\bar{\gamma}_{0}, \quad \text { aber } \quad \gamma\left(\Xi_{\mathrm{L}} \sigma\right) \geq \bar{\gamma}_{0}-\epsilon \quad \text { für kleine } \epsilon>0,
$$

was offenbar ein Widerspruch ist. Also gilt $n_{1}=n_{2}$, und die Funktion $d(\bar{\gamma})$ ist linksseitig stetig. Ihr qualitativer Verlauf ist in Abbildung 4.1 gezeigt. Bezeichnen wir die Unstetigkeitsstellen mit $\bar{\gamma}_{1}<\bar{\gamma}_{2}<\bar{\gamma}_{3}<\ldots$, dann reicht es zur Analyse also meist aus, die Kette

$$
\Phi_{\bar{\gamma}_{1}} \longleftrightarrow \Phi_{\bar{\gamma}_{2}} \longleftrightarrow \Phi_{\bar{\gamma}_{3}} \longleftrightarrow \cdots
$$

zu betrachten.

$\mathrm{Zu}$ klären bleibt, wie sich die Approximationseigenschaft „, $\gamma(\Xi-\psi) \geq \bar{\gamma}^{\text {“ }}$ in den neu formulierten Rahmen übersetzt. Wir führen dazu den Begriff der regulären Projektion auf den endlichdimensionalen Raum $\Phi_{\bar{\gamma}}$ ein. Dieser ist in Anhang $4 . A$ näher erläutert; wir bemerken hier nur, daß es sich dabei um einen Projektionsoperator auf $\Phi_{\bar{\gamma}}$ handelt (also $p: \bar{\Sigma}^{*} \rightarrow \bar{\Sigma}^{*}$, Bild $p=\Phi_{\bar{\gamma}}, p^{2}=p$ ), der sich in der Form

$$
p=\sum_{j=1}^{J} \sigma_{j}(\cdot) \phi_{j} \quad \text { mit } \sigma_{j} \in \Sigma(E) \text { und } \phi_{j} \in \Phi_{\bar{\gamma}}
$$


Abbildung 4.1: Qualitativer Verlauf der Punktfeldraum-Dimensionen

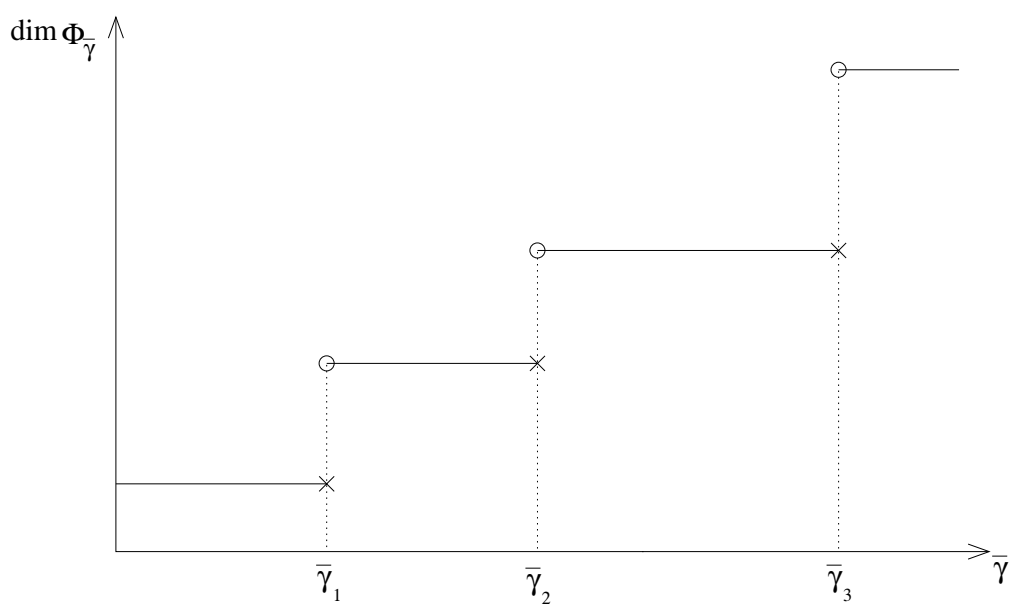

schreiben läßt ( $E, J$ geeignet). Eine solche reguläre Projektion existiert zu jedem endlichdimensionalen Teilraum von $\bar{\Sigma}^{*}$ (siehe Satz 4.11 in Anhang 4.A), muß aber nicht eindeutig sein.

Mit Hilfe dieser Projektionen läßt sich die Approximationseigenschaft umformulieren. Wir schreiben dazu etwas symbolisch $p_{\bar{\gamma}} \Xi=\Xi\left(p_{\bar{\gamma}^{*}} \cdot, \cdot\right)$ usw. und zeigen:

Satz 4.2. Sei $p_{\bar{\gamma}}$ ein regulärer Projektor auf $\Phi_{\bar{\gamma}}$, dann gilt

$$
\gamma\left(\left\|\Xi-p_{\bar{\gamma}} \Xi\right\|_{E, r}\right) \geq \bar{\gamma} \quad \forall E>0 .
$$

Hierbei ist $\|\cdot\|_{E, r}$ bei festem $E$ als Funktion von $r$ zu lesen.

Beweis. Wir wählen vermöge des Phasenraumkriteriums ein $\psi \in \bar{\Psi}_{0}^{P}$, so daß $\gamma(\Xi-\psi) \geq \bar{\gamma}$. Beachten wir Satz [3.2, dann können wir nach eventuellem Weglassen des "sekundären“ direkten Summanden annehmen, daß $\operatorname{Bild}_{\mathrm{R}} \psi=\Phi_{\bar{\gamma}}$, und daß bei festem $E$ gilt

$$
\gamma\left(\|\Xi-\psi\|_{E, r}\right) \geq \bar{\gamma} \text {. }
$$

Die reguläre Projektion $p_{\bar{\gamma}}$ schreiben wir als $p_{\bar{\gamma}}=\sum_{j=1}^{J} \sigma_{j}(\cdot) \phi_{j}$ mit $\sigma_{j} \in \Sigma\left(E^{\prime}\right)\left(E^{\prime}\right.$ geeignet), $\phi_{j} \in \Phi_{\bar{\gamma}}$. Dann folgt, daß

$$
\begin{aligned}
\left\|p_{\bar{\gamma}}(\Xi-\psi)\right\|_{E, r}=\| \sum_{j=1}^{J}(\Xi & -\psi)\left(\sigma_{j}, \cdot\right) \phi_{j} \|_{E, r} \\
& \leq \sum_{j=1}^{J}\left\|\sigma_{j}\right\|\left\|\phi_{j}\right\|_{E}\|\Xi-\psi\|_{E^{\prime}, r} \leq\|\Xi-\psi\|_{E^{\prime}, r} \cdot \text { const. }
\end{aligned}
$$

Zusammen mit (4.1.17) ergibt das also

$$
\gamma\left(\left\|p_{\bar{\gamma}}(\Xi-\psi)\right\|_{E, r}\right) \geq \bar{\gamma} \quad \forall E>0 .
$$

Wegen $\operatorname{Bild}_{\mathrm{R}} \psi=\Phi_{\bar{\gamma}}$ gilt $p_{\bar{\gamma}} \psi=\psi$; damit folgt die Behauptung per Dreiecksungleichung aus (4.1.17) und (4.1.19). 


\subsection{Gruppendarstellungen}

Wir interessieren uns nun für Darstellungen von Symmetriegruppen auf den Punktfeldräumen $\Phi_{\bar{\gamma}}$; dies ist unter anderem zur Nachprüfung der Wightman-Axiome von Bedeutung. Die Wirkung solcher Symmetrieoperationen, wie etwa der Lorentzgruppe円, der Involution $A \mapsto A^{*}$ oder der Dilatationsgruppe, sind auf $\mathfrak{B}(\mathcal{H})$ bereits vorgegeben. Die Frage ist nun, wie diese Operationen auf die Räume $\Phi_{\bar{\gamma}}$ wirken und insbesondere, ob sie diese in sich überführen; in diesem Fall können wir dann von einer Kovarianz der konstruierten Punktfelder sprechen. Das Prinzip der Übertragung solcher Operationen auf die $\Phi_{\bar{\gamma}}$ wird in diesem Abschnitt erläutert.

Wir befassen uns zunächst mit einer einzigen Transformation und ignorieren eine eventuell vorhandene Gruppenstruktur. Für unsere Zwecke soll solch eine Transformation durch eine Abbildung

$$
\alpha: \mathfrak{B}(\mathcal{H}) \rightarrow \mathfrak{B}(\mathcal{H})
$$

gegeben sein, von der wir voraussetzen, daß sie linearf, invertierbar und schwach-*-stetig ist. Außerdem sei sie isometrisch, d.h. es gelte $\|\alpha B\|=\|B\| \forall B \in \mathfrak{B}(\mathcal{H})$. In den Anwendungen sind solche Operationen typischerweise gegeben durch

$$
\alpha B=U B U^{*}
$$

mit einem unitären Operator $U \in \mathfrak{B}(\mathcal{H})$. Solch ein $\alpha$ erfüllt offenbar die geforderten Bedingungen.

Da wir die schwach-*-Stetigkeit von $\alpha$ gefordert hatten, können wir $\alpha$ auf den Prädualraum $\Sigma$ von $\mathfrak{B}(\mathcal{H})$, liften“, indem wir setzen

$$
\alpha_{*} \sigma=\sigma \circ \alpha \quad \text { bzw. } \quad \alpha_{*} \sigma(B)=\sigma(\alpha B) \quad(\sigma \in \Sigma, B \in \mathfrak{B}(\mathcal{H})) .
$$

Allerdings werden $\alpha$ und $\alpha_{*}$ im allgemeinen nicht verträglich sein mit den Garbenstrukturen von $\bar{\Sigma}$ bzw. $\overline{\mathfrak{A}}$. Um die Transformation in diesem Rahmen behandeln zu können, müssen wir zusätzlich fordern, daß $\alpha$ die Lokalisierung in Orts- und Impulsraum in gewisser Weise respektiert. Es ist sicher nicht vorteilhaft, dabei zu verlangen, daß etwa

$$
\alpha \mathfrak{A}(r) \subset \mathfrak{A}(r) \quad \forall 0<r<1,
$$

denn dies würde z.B. Lorentz-Boosts oder Dilatationen ausschließen. Wir schwächen daher (4.2.4) in folgender Definition etwas ab:

Definition 4.3. Eine lineare (oder antilineare) Abbildung $\alpha: \mathfrak{B}(\mathcal{H}) \rightarrow \mathfrak{B}(\mathcal{H})$ heiße lokale Transformation, wenn sie invertierbar und isometrisch ist, stetig bzgl. der schwach-*Topologie auf $\mathfrak{B}(\mathcal{H})$, und wenn es ein $\lambda \geq 1$ gibt, so daß

$$
\alpha \mathfrak{A}(r) \subset \mathfrak{A}(\lambda r) \quad \forall 0<r<1, \lambda r<1
$$

und

$$
\alpha_{*} \Sigma(E) \subset \Sigma(\lambda E) \quad \forall E>1, \lambda E>1
$$

\footnotetext{
1 Translationen bilden hier einen Sonderfall und werden erst in Abschnitt 4.3 behandelt, so daß erst dort die Kovarianz der Felder unter der vollen Poincaré-Gruppe untersucht werden kann.

${ }^{2}$ Wir werden unten eine Verallgemeinerung auf antilineare Transformationen diskutieren.
} 
Ein solches $\alpha$ können wir dann als Abbildung auf der Präkogarbe $\overline{\mathfrak{A}}$ auffassen: Wir erhalten Abbildungen $\alpha_{r r^{\prime}}: \mathfrak{A}(r) \rightarrow \mathfrak{A}\left(r^{\prime}\right)$, wenn $r^{\prime}$ genügend groß gewählt ist $\left(r^{\prime} / r \geq\right.$ $\lambda$ ), und diese sind sicher verträglich mit den Garbeninklusionen. Analoges gilt für die Präkogarbe $\bar{\Sigma}$.

Dementsprechend können wir auch von einer Wirkung von $\alpha$ auf $\bar{\Sigma}^{*}$ sprechen: Ein $\phi \in \bar{\Sigma}^{*}$ ist gegeben durch eine Linearform $\phi_{E}$ auf jedem $\Sigma(E)$, und $\alpha \phi_{E}: \Sigma(\lambda E) \rightarrow \mathbb{C}$ bildet dann auch eine wohldefinierte Linearform auf $\bar{\Sigma}$. (Dabei setzen wir ggf. durch die Garbenabbildungen zu „kleinen“ $E$ fort.) Ohne direkt auf die Garbenstruktur Bezug zu nehmen, können wir also $\alpha$ einfach als Abbildung auf dem Vektorraum $\bar{\Sigma}^{*}$ betrachten.

Wir wollen nach der Klärung dieser Begrifflichkeiten nun untersuchen, wie $\alpha$ auf $\Phi_{\bar{\gamma}}$ wirkt, und insbesondere zeigen, daß $\alpha \Phi_{\bar{\gamma}} \subset \Phi_{\bar{\gamma}}$. Dazu übertragen wir die Wirkung von $\alpha$ zunächst auf die Prädualräume.

Wir hatten schon gesehen, in welchem Sinne die präduale Abbildung $\alpha_{*}$ auf der Präkogarbe $\bar{\Sigma}$ bzw. auf die Räume $\Sigma(E)$ wirkt. Zusammengefaßt haben wir dort folgende Situation:

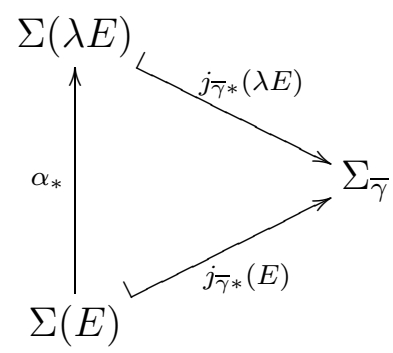

Hierbei wissen wir nach Lemma 4.1, daß

$$
\operatorname{Kern} j_{\bar{\gamma}^{*}}(E)=\{\sigma \in \Sigma(E) \mid \gamma(\sigma) \geq \bar{\gamma}\}
$$

Nun gilt aber wegen der Isometrie von $\alpha$, daß

$$
\left\|\alpha_{*} \sigma\right\|_{r}=\|\sigma \circ \alpha\|_{r} \leq\|\sigma\|_{\lambda r} \quad \text { für kleine } r,
$$

und deshalb wird nach (2.D.11):

$$
\gamma\left(\alpha_{*} \sigma\right) \geq \gamma(\sigma)
$$

Wir haben also $\alpha_{*} \operatorname{ker} j_{\bar{\gamma} *}(E) \subset \operatorname{ker} j_{\bar{\gamma} *}(\lambda E)$, und damit liefert $\alpha_{*}$ eine wohldefinierte lineare Abbildung auf dem „Quotientenraum“ $\Sigma_{\bar{\gamma}}$, die wir mit $\check{\alpha}_{*}$ bezeichnen. Wir können diese wiederum per Dualität auf den Dualraum $\Sigma_{\bar{\gamma}}^{*}=\Phi_{\bar{\gamma}}$ übertragen und erhalten so einen Operator $\check{\alpha}: \Phi_{\bar{\gamma}} \rightarrow \Phi_{\bar{\gamma}}$. Insgesamt haben wir vier "Darsteller" von $\alpha$ erhalten, die wir symbolisch so zusammenfassen:

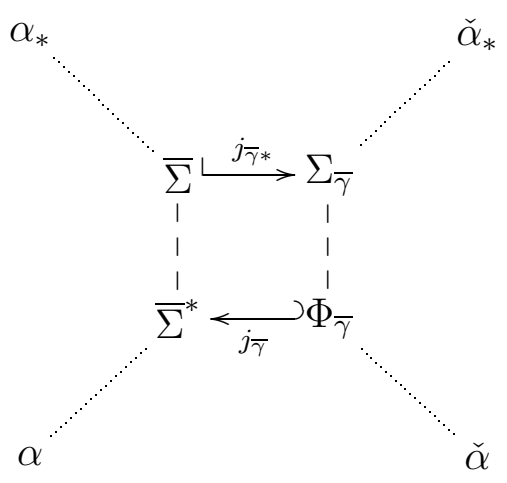


Man rechnet aus den Definitionen nun leicht nach, daß gilt

$$
j_{\bar{\gamma}} \circ \check{\alpha}=\alpha \circ j_{\bar{\gamma}}
$$

oder daß, wenn wir $\Phi_{\bar{\gamma}}$ als Teilraum von $\bar{\Sigma}^{*}$ begreifen, dieser stabil unter der Wirkung von $\alpha$ ist. Dies ist die gewünschte Ergebnis.

Wir erhalten solch einen Operator $\check{\alpha}$ natürlich für jedes $\bar{\gamma}$ (das oben festgehalten war), wobei per Konstruktion klar ist, daß diese verschiedenen Operatoren mit den Inklusionen $\Phi_{\bar{\gamma}} \hookrightarrow \Phi_{\bar{\gamma}^{\prime}}\left(\bar{\gamma}<\bar{\gamma}^{\prime}\right)$ verträglich sind. Wir können also auch von einem Operator auf $\Phi_{\mathrm{FH}}$ sprechen, der jedes $\Phi_{\bar{\gamma}}$ in sich überführt.

Wir bemerken noch, daß sich obige Überlegungen genauso auch für antilineare Operatoren $\alpha$ formulieren lassen, wobei der präduale Operator $\alpha_{*}$ hier abweichend definiert wird durch

$$
\left(\alpha_{*} \sigma\right)(\phi):=\overline{\sigma(\alpha \phi)}, \quad \sigma \in \bar{\Sigma}, \phi \in \bar{\Sigma}^{*}
$$

analog modifiziert man die Definition von $\check{\alpha}$ durch $\check{\alpha}_{*}$. Damit haben wir insgesamt folgenden Satz bewiesen:

Satz 4.4. Es sei $\alpha$ eine lokale Transformation (linear oder antilinear). Dann gibt es einen Operator $\check{\alpha}$ auf $\Phi_{\mathrm{FH}}$, der jedes $\Phi_{\bar{\gamma}}$ stabil läßt und der

$$
j_{\bar{\gamma}} \circ \check{\alpha}=\alpha \circ j_{\bar{\gamma}}
$$

erfüllt, d.h. als Teilräume von $\bar{\Sigma}^{*}$ sind die $\Phi_{\bar{\gamma}}$ stabil unter $\alpha$.

Da die Transformationen $\check{\alpha}$ auf den $\Phi_{\bar{\gamma}}$ im diskutierten Sinne nichts anderes sind als Restriktionen von $\alpha$ auf Teilräume von $\bar{\Sigma}^{*}$, ist sofort klar, daß die Zuordnung $\alpha \mapsto \check{\alpha}$ nicht nur linear ist, sondern auch die Produktstruktur erhält $\left((\alpha \beta)^{\swarrow}=\check{\alpha} \check{\beta}\right)$. Ist also $g \mapsto \alpha(g)$ eine Darstellung einer Gruppe $G$ auf $\mathfrak{B}(\mathcal{H})$, wobei die Darsteller $\alpha(g)$ lokale Transformationen sind, so erhalten wir unmittelbar Darstellungen $g \mapsto \check{\alpha}(g)$ der Gruppe auf den $\Phi_{\bar{\gamma}}$.

Entsprechend ergeben sich auch Darstellungen der Gruppe auf $\bar{\Sigma}$ und $\Sigma_{\bar{\gamma}}$, wobei wir allerdings $\alpha_{\times}(g):=\alpha_{*}\left(g^{-1}\right)$ als Darsteller auf $\bar{\Sigma}$ verwenden müssen. Wir werden diese Darstellungen aber hier nicht weiter betrachten.

Von Interesse ist noch die Stetigkeit der Darstellung $g \mapsto \check{\alpha}(g)$ auf $\Phi_{\bar{\gamma}}$. Dazu versehen wir den Raum der linearen Abbildungen $\alpha: \Sigma(E)^{*} \rightarrow \Sigma\left(E^{\prime}\right)^{*}$ mit folgenden Halbnormen:

$$
p_{\xi, \xi^{\prime}, A}(\alpha)=\left|\left(\xi \mid \alpha A \xi^{\prime}\right)\right|, \quad \xi, \xi^{\prime} \in P\left(E^{\prime}\right) \mathcal{H}, A \in \Sigma(E)^{*} .
$$

Das liefert eine lokal konvexe, hausdorffsche Topologie auf diesem Raum von Abbildungen.

Wir betrachten nun speziell den (für die Anwendungen wichtigen) Fall, daß die $\alpha(g)$ von einer stark stetigen unitären Darstellung $g \mapsto U(g)$ der Gruppe $G$ auf $\mathcal{H}$ herrühren. Es gelte außerdem

$$
U(g) P(E) \mathcal{H} \subset P(\lambda E) \mathcal{H} \quad \forall E \geq 1 \text { mit in } g \text { lokal gleichmäßig wählbarem } \lambda \text {; }
$$

das impliziert dann gerade die geforderte Verträglichkeit der $\alpha(g)$ mit der Energiebeschränkung. Mit $E^{\prime}=\lambda E$ ( $\lambda$ fest auf einer Umgebung von $\mathbf{1}_{G}$ ) erhalten wir in diesem Fall

$$
p_{\xi, \xi^{\prime}, A}(\alpha(g)-\mathbf{1}) \leq\left|\left(\xi \mid(U(g)-\mathbf{1}) A U(g)^{*} \xi^{\prime}\right)\right|+\left|\left(\xi \mid A\left(U(g)^{*}-\mathbf{1}\right) \xi^{\prime}\right)\right|,
$$


was wegen der starken Stetigkeit von $U(g)$ für $g \rightarrow \mathbf{1}_{G}$ verschwindet. Die Zuordnung $g \mapsto \alpha(g)$ ist also in der betrachteten Topologie stetig. Eingeschränkt auf den endlichdimensionalen Raum der linearen Abbildungen $\Phi_{\bar{\gamma}} \rightarrow \Phi_{\bar{\gamma}}$ stimmt diese aber mit der Standardtopologie überein, so daß $g \mapsto \check{\alpha}(g)$ stetig im üblichen Sinne ist. Unser Ergebnis lautet also:

Satz 4.5. Sei $\alpha$ eine Darstellung einer Gruppe $G$ auf $\mathfrak{B}(\mathcal{H})$, und die Darsteller $\alpha(g)(g \in$ $G)$ seien lokale Transformationen. Dann liefert die Restriktion von $\alpha$ auf $\Phi_{\mathrm{FH}} \subset \bar{\Sigma}^{*}$ dort eine Darstellung $\check{\alpha}$ von $G$, die jedes $\Phi_{\bar{\gamma}}$ stabil läßt. Ist speziell

$$
\alpha(g)=U(g) \cdot U(g)^{*}
$$

mit einer stark stetigen unitären Darstellung $U(g)$ von $G$ auf $\mathcal{H}$, und gilt

$$
U(g) P(E) \mathcal{H} \subset P(\lambda E) \mathcal{H} \quad \forall E \geq 1
$$

mit zu g lokal gleichmäßig wählbarem $\lambda$, dann ist $g \mapsto \check{\alpha}(g)$ auf jedem $\Phi_{\bar{\gamma}}$ eine stetige Darstellung.

Wir wollen noch die Transformation von regulären Projektionen auf $\Phi_{\bar{\gamma}}$ betrachten, die ja in unserem Kontext die „Phasenraumapproximation“ realisieren. Es sei $p_{\bar{\gamma}}$ eine solche Projektion. Auch auf sie wirken die Symmetrietransformationen in natürlicher Weise, nämlich durch Adjunktion:

$$
p_{\bar{\gamma}}^{\prime}=\alpha \circ p_{\bar{\gamma}} \circ \alpha^{-1}
$$

Wir zeigen, daß diese Operation reguläre Projektionen wieder in solche überführt.

Satz 4.6. Sei $\alpha$ eine lokale Transformation. Ist $p_{\bar{\gamma}}$ eine reguläre Projektion auf $\Phi_{\bar{\gamma}}$, so auch $p_{\bar{\gamma}}^{\prime}:=\alpha \circ p_{\bar{\gamma}} \circ \alpha^{-1}$.

Beweis. Da $\alpha \Phi_{\bar{\gamma}}=\Phi_{\bar{\gamma}}$ gilt, ist Bild $p_{\bar{\gamma}}^{\prime}=\Phi_{\bar{\gamma}}$. Die Eigenschaft $\left(p_{\bar{\gamma}}^{\prime}\right)^{2}=p_{\bar{\gamma}}^{\prime}$ folgt sofort aus $p_{\bar{\gamma}}^{2}=p_{\bar{\gamma}}$. Die Stetigkeitseigenschaft von $p_{\bar{\gamma}}^{\prime}$ ist ebenfalls gegeben, da $\alpha^{-1}$ die Energiebeschränktheit von Funktionalen respektiert.

Wir kommen nun zu den angekündigten Anwendungen.

Lorentz-Transformationen Sind $U(\Lambda)$ die Darsteller der Lorentztransformationen auf $\mathfrak{B}(\mathcal{H})$, so sind $\alpha(\Lambda)=$ ad $U(\Lambda)$ lokale Transformationen. Nachzuprüfen bleiben dabei nur die Lokalitätsbedingungen: Die Ortsraumlokalisierung bleibt wegen

$$
\alpha(\Lambda) \mathfrak{A}(r) \subset \mathfrak{A}\left(\Lambda \mathcal{O}_{r}\right) \subset \mathfrak{A}(2\|\Lambda\| r)
$$

erhalten. Im Impulsraum hat man

$$
P(E) U(\Lambda)=U(\Lambda) U(\Lambda)^{*} P(E) U(\Lambda)=U(\Lambda) P^{\Lambda}(E)
$$

hier ist $P^{\Lambda}(E)$ der Energieprojektor in einem lorentztransformierten Bezugssystem. Da sich das gemeinsame Spektrum der $P_{\mu}$ mit der bekannten $(s+1)$-dimensionalen Darstellung von $\mathfrak{L}$ transformiert, erhält man für genügend groß gewähltes $\lambda$

$$
P^{\Lambda}(E) P(\lambda E)=P^{\Lambda}(E) .
$$


Dies impliziert nach (4.2.17), daß

$$
P(E) U(\Lambda) P(\lambda E)=P(E) U(\Lambda) \quad \Rightarrow \quad U(\Lambda) P(E) \mathcal{H} \subset P(\lambda E) \mathcal{H}
$$

Wir können hier also Satz 4.5 anwenden und erhalten bei festem $\bar{\gamma}$ eine stetige endlichdimensionale Darstellung $\check{\alpha}$ von $\mathfrak{L}$ auf $\Phi_{\bar{\gamma}}$. Entwickeln wir sie nach einer Basis $\left\{\phi_{1}, \ldots, \phi_{J}\right\}$ von $\Phi_{\bar{\gamma}}$, dann erhalten wir eine stetige Matrixdarstellung $S_{j k}(\Lambda)$ von $\mathfrak{L}$, so daß

$$
U(\Lambda) \phi_{j} U(\Lambda)^{*}=\sum_{k=1}^{J} S_{j k}\left(\Lambda^{-1}\right) \phi_{k}
$$

im Sinne von Linearformen auf $\bar{\Sigma}$. Die Darstellung ist vollständig reduzibel [Boe55, IX §3]; durch Aufspaltung von $\Phi_{\bar{\gamma}}$ in irreduzible Teilräume kann man einzelne Skalar- und Vektorfelder sowie Tensorfelder höherer Ordnung identifizieren [Cor84, 17.2].

Zur Überprüfung der Wightman-Axiome ist Gleichung (4.2.20) noch auf die ausintegrierten Felder zu übertragen. Man hat für $\sigma \in \Sigma(E)$ :

$$
\begin{gathered}
\sigma\left(U(\Lambda) \phi_{j}(f) U(\Lambda)^{*}\right)=\int d^{s+1} x f(x) \sigma\left(U(\Lambda) U(x) \phi_{j} U(x)^{*} U(\Lambda)^{*}\right) \\
\stackrel{(1.2 .20)}{=} \sum_{k=1}^{J} S_{j k}\left(\Lambda^{-1}\right) \int d^{s+1} x f(x) \sigma\left(U(\Lambda x) \phi_{k} U(\Lambda x)^{*}\right) \\
=\sum_{k=1}^{J} S_{j k}\left(\Lambda^{-1}\right) \int d^{s+1} x f\left(\Lambda^{-1} x\right) \sigma\left(U(x) \phi_{k} U(x)^{*}\right) \\
=\sum_{k=1}^{J} S_{j k}\left(\Lambda^{-1}\right) \sigma\left(\phi_{k}\left(f_{\Lambda}\right)\right), \quad \text { wobei } f_{\Lambda}(y)=f\left(\Lambda^{-1} y\right) .
\end{gathered}
$$

Dies läßt sich stetig auf Vektorfunktionale $\sigma=\left(\xi \mid \cdot \xi^{\prime}\right)$ mit $\xi \in \mathcal{H}, \xi^{\prime} \in \mathcal{C}^{\infty}(\mathcal{H})$ erweitern, d.h. man hat

$$
U(\Lambda) \phi_{j}(f) U(\Lambda)^{*}=\sum_{k=1}^{J} S_{j k}\left(\Lambda^{-1}\right) \phi_{k}\left(f_{\Lambda}\right)
$$

als Operatorgleichung auf $\mathcal{C}^{\infty}(\mathcal{H})$.

Hermitesche Konjugation Als weitere Anwendung betrachten wir die antilineare Abbildung $\alpha: A \mapsto A^{*}$ (,hermitesche Konjugation“). Da $\left\|A^{*}\right\|=\|A\|, \mathfrak{A}(r)^{*}=\mathfrak{A}(r)$ und $(P(E) A P(E))^{*}=P(E) A^{*} P(E)$, ist $\alpha$ eine lokale Transformation. Die induzierte Wirkung auf ein $\phi \in \bar{\Sigma}^{*}$ stellt sich, ausgewertet zwischen Vektoren $\xi, \xi^{\prime} \in P(E) \mathcal{H}$, folgendermaßen dar:

$$
\left(\xi \mid(\alpha \phi) \xi^{\prime}\right)=\left(\xi^{\prime} \mid \phi \xi\right)=\left(\xi \mid \phi^{*} \xi^{\prime}\right) ;
$$

hier ist $\phi^{*}$ die zu $\phi$ adjungierte quadratische Form. Wir wissen also nach Satz 4.4, daß mit jedem Feld $\phi \in \Phi_{\gamma}$ auch die adjungierte Form in $\Phi_{\bar{\gamma}}$ liegt. Dies überträgt sich sofort auf die Operatoren $\phi(f)$ und ihre Adjungierten, denn wir wissen bereits aus (3.4.7), daß

$$
\phi(\bar{f})^{*}=\phi^{*}(f) \text { als Gleichung auf } \mathcal{C}^{\infty}(\mathcal{H}) .
$$


Wählen wir nun eine Basis von $\Phi_{\bar{\gamma}}$ aus $\alpha$-invarianten Vektoren $\phi_{j}$, dann ist mit $\phi_{j}(f)$ auch $\phi_{j}(\bar{f})^{*}$ in der Menge $\left\{\phi_{1}(f), \ldots, \phi_{J}(f)\right\}$ enthalten, wie dies die Wightman-Axiome auch fordern.

(Die Wahl einer solchen Basis ist immer möglich: Ist $\left\{\phi_{1}, \ldots, \phi_{J}\right\}$ eine beliebige Basis von $\Phi_{\bar{\gamma}}$, dann spannen die $2 J$ Vektoren

$$
\phi_{j}^{+}:=\left(\phi_{j}+\check{\alpha} \phi_{j}\right), \quad \phi_{j}^{-}:=\frac{1}{i}\left(\phi_{j}-\check{\alpha} \phi_{j}\right)
$$

ebenfalls den Raum $\Phi_{\bar{\gamma}}$ auf, sind aber $\alpha$-invariant. Wir können nun aus ihnen eine neue Basis auswählen.)

Dilatationen Wir diskutieren noch den Fall, daß die betrachtete Feldtheorie dilatationsinvariant ist, d.h. daß wir als zusätzliche Struktur auf $\mathfrak{B}(\mathcal{H})$ eine stark stetige unitäre Darstellung $U(\lambda)$ der Dilatationsgruppe gegeben haben, die geometrisch auf den lokalen Algebren wirkt:

$$
\alpha(\lambda) \mathfrak{A}(r)=\mathfrak{A}(\lambda r),
$$

wobei wieder $\alpha(\lambda)=\operatorname{ad} U(\lambda)$. Die Vertauschungsrelationen zwischen Lorentz- und Dilatationsgruppe bedingen, daß

$$
U(\lambda) P(E) U(\lambda)^{*}=P(E / \lambda) \quad \Rightarrow \quad U(\lambda) P(E) \mathcal{H} \subset P(E / \lambda) \mathcal{H}
$$

Damit ist $\alpha(\lambda)$ eine lokale Transformation, wobei man in Definition 4.3 ggf. $\lambda$ durch 1 ersetzt. Wir erhalten also eine stetige Darstellung $\check{\alpha}$ der Dilatationsgruppe auf jedem $\Phi_{\bar{\gamma}}$. Die endlichdimensionale Darstellungstheorie dieser Gruppe - die durch Logarithmieren des Parameters zur Gruppe $(\mathbb{R},+)$ isomorph ist - ist vollständig bekannt [Boe55, V §9]; der Darstellungsraum läßt sich in Unterräume zerlegen, auf denen die Darsteller als Matrizen der folgenden Form wirken:

$$
\check{\alpha}(\lambda)=\lambda^{q}\left(\begin{array}{ccccc}
1 & 0 & 0 & \ldots & 0 \\
\log \lambda & 1 & 0 & \ldots & 0 \\
\frac{(\log \lambda)^{2}}{2 !} & \log \lambda & 1 & \ldots & 0 \\
\vdots & \vdots & & \ddots & \vdots \\
\frac{(\log \lambda)^{n}}{n !} & \frac{(\log \lambda)^{n-1}}{(n-1) !} & \ldots & \log \lambda & 1
\end{array}\right), \quad n \in \mathbb{N}, q \in \mathbb{C} .
$$

Wir können den Feldern also eine wohldefinierte „Skalendimension“ Re $q$ zuordnen.

\subsection{Translationen}

In der gesamten bisherigen Konstruktion haben wir das Verhalten der Theorie am Koordinatenursprung betrachtet und dort lokalisierte Punktfelder $\phi=\phi(0)$ untersucht. Zur Nachprüfung der Wightman-Axiome müssen wir allerdings noch das Verhalten unter Translationen mit einbeziehen. (Diese hatten wir in Abschnitt 4.2 ausgespart, da sie den Koordinatenursprung des Minkowskiraums nicht invariant lassen.) 
Man könnte hierzu das asymptotische Phasenraumkriterium „an jedem Punkt" des Minkowskiraums fordern, entsprechende Feldhalme $\Phi_{\bar{\gamma}}(x)$ konstruieren und Relationen zwischen diesen Räumen für verschiedene $x$ untersuchen. Da die betrachtete Theorie aber translationssymmetrisch ist, erhalten wir dieselbe Struktur viel einfacher, indem wir direkt die unitären Darsteller der Translationen verwenden. Wir definieren für $\phi \in \Phi_{\mathrm{FH}}$ im Sinne von Linearformen auf $\bar{\Sigma}$ :

$$
\phi(x):=U(x) \phi U(x)^{*}, \quad x \in \mathcal{M} .
$$

Wir können dann die Kovarianz der $\phi(x)$ unter der gesamten Poincaré-Gruppe $\mathfrak{P}$ nachweisen: Sei dazu $\left\{\phi_{j}\right\}_{j=1}^{J}$ eine Basis von $\Phi_{\bar{\gamma}}$ bei festem $\bar{\gamma}$. Für $x \in \mathcal{M}, \Lambda \in \mathfrak{L}$ hat man dann als Relation in $\bar{\Sigma}^{*}$ :

$$
\begin{gathered}
U(x, \Lambda) \phi_{j}(y) U(x, \Lambda)^{*}=U(x) U(\Lambda) U(y) \phi_{j} U(y)^{*} U(\Lambda)^{*} U(x)^{*} \\
=U(x) U(\Lambda y) U(\Lambda) \phi_{j} U(\Lambda)^{*} U(\Lambda y)^{*} U(x)^{*} \stackrel{(4.2 .20)}{=} U(\Lambda y+x) \sum_{k=1}^{J} S_{j k}\left(\Lambda^{-1}\right) \phi_{k} U(\Lambda y+x)^{*} \\
=\sum_{k=1}^{J} S_{j k}\left(\Lambda^{-1}\right) \phi_{k}(\Lambda y+x) . \quad(4.3 .2)
\end{gathered}
$$

Dies ist gerade die gewünschte Kovarianzeigenschaft. Durch Integration mit einer Testfunktion - ähnlich wie in (4.2.21) - erhält man ebenso

$$
U(x, \Lambda) \phi_{j}(f) U(x, \Lambda)^{*}=\sum_{k=1}^{J} S_{j k}\left(\Lambda^{-1}\right) \phi_{k}\left(f_{x, \Lambda}\right) \quad \text { mit } \quad f_{x, \Lambda}(y)=f\left(\Lambda^{-1}(y-x)\right)
$$

zunächst im Sinne von Linearformen auf $\bar{\Sigma}$, dann durch Erweiterung als Operatorgleichung auf $\mathcal{C}^{\infty}(\mathcal{H})$.

Zusammen mit den Ergebnissen der vorangegangenen Abschnitte haben wir damit alle Wightman-Axiome für die Elemente von $\Phi_{\bar{\gamma}}$ nachgeprüft, mit Ausnahme der Invarianz der Wightman-Domäne $\mathcal{D}=\mathcal{C}^{\infty}(\mathcal{H})$ unter Poincaré-Transformationen. Man bemerkt dazu, daß wegen der Gruppenrelationen gilt

$$
U(x, \Lambda) \frac{1}{1+H} U(x, \Lambda)^{*}=\frac{1}{1+\Lambda_{0}^{\mu} P_{\mu}}
$$

und folglich für $\xi \in \mathcal{H}$ und $l \in \mathbb{N}_{0}$ :

$$
U(x, \Lambda)\left(\frac{1}{1+H}\right)^{l} \xi=\left(\frac{1}{1+\Lambda_{0}^{\mu} P_{\mu}}\right)^{l} U(x, \Lambda) \xi .
$$

Damit wird

$$
U(x, \Lambda) R^{l} \xi=R^{l}\left(\frac{1+H}{1+\Lambda_{0}^{\mu} P_{\mu}}\right)^{l} U(x, \Lambda) \xi \in R^{l} \mathcal{H} \quad \Rightarrow \quad U(x, \Lambda) \mathcal{C}^{\infty}(\mathcal{H}) \subset \mathcal{C}^{\infty}(\mathcal{H})
$$


sofern wir noch zeigen können, daß $\frac{1+H}{1+\Lambda_{0}^{\mu} P_{\mu}}$ als beschränkter Operator existiert. Dazu bemerkt man, daß es $\epsilon>0$ gibt mit

$$
\Lambda_{0}^{\mu} P_{\mu} \geq \epsilon H .
$$

Das ist zumindest in dem Fall klar, daß $\Lambda$ einen boost entlang der 1-Achse darstellt, denn dann ist mit gewissem $\chi \in \mathbb{R}$

$$
\Lambda_{0}^{\mu} P_{\mu}=\cosh \chi P_{0}+\sinh \chi P_{1} \geq \cosh \chi H-|\sinh \chi|\left|P_{1}\right| \geq \underbrace{(\cosh \chi-|\sinh \chi|)}_{=: \epsilon} H
$$

unter Verwendung der Spektrumsbedingung. Allgemeine Lorentztransformationen ergeben sich aus solchen $\Lambda$ durch Komposition mit räumlichen Drehungen, die aber die 0Komponenten der Minkowskivektoren nicht ändern. Wir erhalten also auch im allgemeinen Fall

$$
\frac{1}{1+\Lambda_{0}^{\mu} P_{\mu}} \leq \frac{1}{1+\epsilon H}
$$

Da sicher $\frac{1+x}{1+\epsilon x} \leq 1+\frac{1}{\epsilon}$ für $x \in \mathbb{R}_{0}^{+}$, folgt

$$
\frac{1+H}{1+\Lambda_{0}^{\mu} P_{\mu}} \leq \frac{1+H}{1+\epsilon H} \leq \text { const. } \Rightarrow\left\|\frac{1+H}{1+\Lambda_{0}^{\mu} P_{\mu}}\right\|<\infty .
$$

Für die obige Argumentation ist es natürlich wesentlich, daß die Operatoren $P_{\mu}$ eine gemeinsame Spektralschar besitzen, so daß man die Abschätzungen von reellen Zahlen direkt auf Operatoren übertragen kann.

Zusammenfassend können wir nun festhalten:

Satz 4.7. Wir betrachten eine Quantenfeldtheorie, die das asymptotische Phasenraumkriterium mit polynomialen Energieschranken erfüllt. Dann gibt es zu jedem $\bar{\gamma}>0$ eine Basis $\left\{\phi_{1}, \ldots, \phi_{J}\right\}$ von $\Phi_{\bar{\gamma}}$, die (nach Ausintegration) einen Satz von Wightman-Feldern im Sinne der in Abschnitt 1.3.8 aufgeführten Axiome bildet.

Dabei haben wir das in Abschnitt 1.3.2 erwähnte Irreduzibilitätsaxiom nicht betrachtet; durch eine geeignete Einschränkung des Hilbertraums läßt sich aber auch dieses Axiom immer erfüllen. (Wir werden in Abschnitt 8.2 noch näher auf diesen Aspekt eingehen.)

Damit lassen sich alle bekannten Resultate der Wightman-Theorie auch auf die von uns konstruierten Punktfelder anwenden, wie etwa das PCT-Theorem oder der Satz von Reeh und Schlieder. Da wir zusätzlich wissen, daß die Felder zum lokalen Netz affiliiert sind, gilt z.B. auch das Bisognano-Wichmann-Theorem, wenn man annimmt, daß das Vakuum zyklisch für die Felder ist (d.h. eine Einschränkung des Hilbertraums nicht notwendig ist).

\subsection{Differentiation der Felder}

Im Rahmen des Punktfeldformalismus in der Quantenfeldtheorie betrachtet man auch Ableitungen der Punktfelder $\phi(x)$, etwa um Feldgleichungen untersuchen zu können oder erhaltene Ströme zu definieren. Man arbeitet also mit Ausdrücken der Form

$$
\frac{\partial}{\partial x^{\mu}} \phi(x)
$$


Es stellt sich die Frage, wie diese Ableitungen in unserem Rahmen zu behandeln sind; insbesondere sollte die Menge $\Phi_{\mathrm{FH}}$ unter der Anwendung von Differentialoperatoren stabil sein, d.h. der Feldinhalt sollte zu jedem Feld $\phi$ auch seine Ableitungen $\partial_{\mu} \phi$ enthalten.

Ähnlich wie die Wirkung der Lorentzgruppe sind auch die Ableitungen der Felder in unserem Rahmen a priori definiert, und zwar unter Verwendung der Translationssymmetrie. Wir hatten die translatierten Felder definiert als

$$
\phi(x):=U(x) \phi U(x)^{*}
$$

mit den Darstellern $U(x)$ der Translationsgruppe. Dann ist mit $P(E) \phi P(E)$ auch $P(E) \phi(x) P(E)$ ein beschränkter Operator. Da die Generatoren $P_{\mu}$ der Translationen nach Multiplikation mit $P(E)$ beschränkt sind (die Spektrumsbedingung liefert $P_{\mu}^{2} \leq H^{2}, \mu=$ $0 \ldots s)$, kann man $U(x)=e^{i P_{\mu} x^{\mu}}$ in (4.4.2) als Potenzreihe ausschreiben, die dann absolut konvergiert:

$$
\begin{aligned}
P(E) \phi(x) P(E) & =\sum_{m, n=0}^{\infty} \frac{i^{m-n}}{m ! n !} P(E)\left(P_{\mu} x^{\mu}\right)^{m} P(E) \phi P(E)\left(P_{\mu} x^{\mu}\right)^{n} P(E) \\
& =P(E) \phi P(E)+i P(E)\left[P_{\mu}, \phi\right] P(E) x^{\mu}+O\left(\|x\|^{2}\right) .
\end{aligned}
$$

Genauer können wir (bei festem $E$ ) schreiben:

$$
\left\|P(E) \phi(x) P(E)-P(E) \phi(0) P(E)-i P(E)\left[P_{\mu}, \phi\right] P(E) x^{\mu}\right\| \leq\|x\|^{2} \cdot \text { const. }
$$

Folglich ist $P(E) \phi(x) P(E)$ bei $x=0$ in der Operatornorm differenzierbar, mit

$$
\left.\frac{\partial}{\partial x^{\mu}} P(E) \phi(x) P(E)\right|_{x=0}=i P(E)\left[P_{\mu}, \phi\right] P(E) .
$$

Im Sinne von Linearformen auf $\bar{\Sigma}$ gilt also

$$
\left.\frac{\partial}{\partial x^{\mu}} \phi(x)\right|_{x=0}=i\left[P_{\mu}, \phi\right] .
$$

Eine andere Möglichkeit, die Ableitungen einzuführen, ist die Differentiation auf Ebene der Wightman-Distributionen:

$$
\left(\partial_{\mu} \phi\right)(f)=-\phi\left(\partial_{\mu} f\right), \quad f \in \mathcal{S}(\mathcal{M}) .
$$

Wir werden unten zeigen, daß dies in unserem Kontext mit der oben gegebenen Definition verträglich ist.

Wir wollen die Differentiation nun in unseren Formalismus einordnen und dabei insbesondere zeigen, daß $\Phi_{\mathrm{FH}} \subset \bar{\Sigma}^{*}$ unter der Wirkung von Differentialoperatoren stabil ist. Es ist möglich, dies direkt im Kontext des Fredenhagen-Hertel-Feldinhalts nachzuweisen, indem man dessen Definition (3.4.1) und die Eigenschaften der approximierenden Folgen aus Satz 3.18 verwendet. Wir wählen hier jedoch einen Zugang, der dem Formalismus der Feldhalme angepaßt ist. Dazu betrachten wir zunächst folgenden Operator auf $\Sigma(E)$ :

$$
\begin{aligned}
D_{\mu *}: & \Sigma(E) \rightarrow \Sigma(E), \\
& D_{\mu *} \sigma(A)=\sigma\left(i\left[P_{\mu}, A\right]\right), \quad A \in \Sigma(E)^{*} .
\end{aligned}
$$


Das ist offenbar verträglich mit den Präkogarben-Inklusionen, liefert also zusammengenommen einen Operator $D_{\mu *}: \bar{\Sigma} \rightarrow \bar{\Sigma}$. Sein dualer Operator $\left(D_{\mu *}\right)^{*} \equiv D_{\mu}$ stimmt nach (4.4.6) gerade mit der eben betrachteten Ableitung überein:

$$
\sigma\left(D_{\mu} \phi\right)=\left.\frac{\partial}{\partial x^{\mu}} \sigma(\phi(x))\right|_{x=0} \quad \forall \sigma \in \Sigma(E) .
$$

Es stellt sich nun die Frage, ob sich $D_{\mu *}$ analog zu den Symmetrietransformationen aus Abschnitt 4.2 auf die Halme $\Sigma_{\bar{\gamma}}$ übertragen läßt. Hierbei ergibt sich eine Schwierigkeit, die dort nicht vorhanden war: Wegen der Relation „ „ $\partial_{\mu} \phi=i\left[P_{\mu}, \phi\right]$ " wird man erwarten, daß das Hochenergieverhalten von $\partial_{\mu} \phi$ um einen Faktor $E$ singulärer ist als das von $\phi$. Folglich sollten wir $\partial_{\mu}$ nicht als Operator $\Phi_{\bar{\gamma}} \rightarrow \Phi_{\bar{\gamma}}$ erhalten, sondern die Ableitung wird $\Phi_{\bar{\gamma}}$ in einen Raum $\Phi_{\bar{\gamma}^{\prime}}$ abbilden, wobei typischerweise $\bar{\gamma}^{\prime} \geq \bar{\gamma}+1$ ist. Dies wirkt sich auf die Konstruktion der verschiedenen Operatoren aus.

Konkret stehen wir dabei vor dem Problem, ob in

$$
\Sigma(E) \stackrel{j_{\bar{\gamma} *}}{\longrightarrow} \Sigma_{\bar{\gamma}}
$$

der Kern von $j_{\bar{\gamma} *}$ stabil unter $D_{\mu *}$ ist. Wie schon angedeutet, ist dies im allgemeinen nicht der Fall. Wir zeigen jedoch, daß wir zu jedem $\bar{\gamma}$ ein $\bar{\gamma}^{\prime}$ finden können, so daß (für jedes feste $E$ )

$$
D_{\mu *} \operatorname{ker} j_{\bar{\gamma} *} \subset \operatorname{ker} j_{\bar{\gamma}^{\prime} *} .
$$

Dies ergibt sich so: Es sei $\sigma \in \Sigma(E)$ fest. Wir haben dann per definitionem

$$
\left\|D_{\mu *} \sigma\right\|_{r}=\sup _{A \in \mathfrak{A}(r)_{1}}\left|\sigma\left(\left[P_{\mu}, A\right]\right)\right| .
$$

Zur Behandlung des Kommutators approximieren wir ihn durch Gleichung (4.4.4). Dazu setzen wir $\alpha_{q}=U\left(q e_{\mu}\right) \cdot U\left(q e_{\mu}\right)^{*}$ für $q>0$, wobei $e_{\mu}$ der Einheitsvektor entlang der $\mu$-Achse ist. Wir erhalten dann für kleine $q$ :

$$
\begin{aligned}
\left|\sigma\left(\left[P_{\mu}, A\right]\right)\right| \leq\left|\sigma\left(\frac{\alpha_{q} A-A}{q}\right)\right|+c \cdot q \leq \frac{1}{q}\|\sigma\|_{q+r}\left\|\alpha_{q} A-A\right\|+c \cdot q & \\
& \leq \frac{2}{q}\|\sigma\|_{q+r}+c \cdot q
\end{aligned}
$$

mit einer Konstanten $c>0$, denn man hat $\alpha_{q} A \in \mathfrak{A}(r+q)$, wenn $A \in \mathfrak{A}(r)$.

Setzen wir nun $q(r)=r^{\beta}$ mit einem $\beta \geq 1$, dann ergibt sich

$$
r^{-\bar{\gamma}+\epsilon}\left\|D_{\mu *} \sigma\right\|_{r} \leq r^{-\bar{\gamma}+\epsilon}\left(\frac{2}{q}\|\sigma\|_{r+q}+c \cdot q\right) \leq r^{-\bar{\gamma}-\beta+\epsilon} \cdot 2\|\sigma\|_{2 r}+r^{-\bar{\gamma}+\beta+\epsilon}
$$

Dies verschwindet für alle $\epsilon>0$, wenn wir annehmen, daß $\beta \geq \bar{\gamma}$ und $\gamma\left(\Xi_{\mathrm{L}} \sigma\right) \geq \bar{\gamma}+\beta$. Setzen wir $\bar{\gamma}^{\prime}:=\bar{\gamma}+\beta$, dann haben wir also die Implikation

$$
\gamma\left(\Xi_{\mathrm{L}} \sigma\right) \geq \bar{\gamma}^{\prime} \Rightarrow \gamma\left(\Xi_{\mathrm{L}} D_{\mu *} \sigma\right) \geq \bar{\gamma}
$$

Nach der Charakterisierung des Kerns der $j_{\bar{\gamma} *}$ aus Lemma 4.1 erhalten wir damit: 
Lemma 4.8. $Z u \bar{\gamma}>0$ gibt es $\bar{\gamma}^{\prime}>0$, so daß

$$
D_{\mu *} \operatorname{ker} j_{\bar{\gamma}^{\prime} *} \subset \operatorname{ker} j_{\bar{\gamma} *}
$$

Explizit kann man $\bar{\gamma}^{\prime}=\max \{\bar{\gamma}+1,2 \bar{\gamma}\}$ wählen.

Das bedeutet, daß wir für genügend großes $\bar{\gamma}^{\prime}$ eine Abbildung $\check{D}_{\mu *}: \Sigma_{\bar{\gamma}^{\prime}} \rightarrow \Sigma_{\bar{\gamma}}$ „,restklassenweise" definieren können:

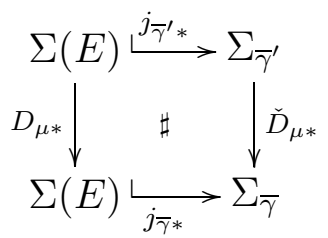

Betrachtet man die dazu duale Abbildung $\check{D}_{\mu} \equiv\left(\check{D}_{\mu *}\right)^{*}$, dann führt diese von $\Phi_{\bar{\gamma}}$ nach $\Phi_{\bar{\gamma}^{\prime}}$ :

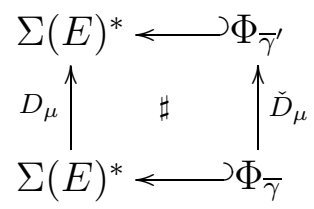

Per Kompatibilität mit den Inklusionsabbildungen können wir $\check{D}_{\mu}$ also als Restriktion von $D_{\mu}$ auf $\Phi_{\mathrm{FH}}$ verstehen, bzw. der Operator $D_{\mu}$ läßt den Feldinhalt $\Phi_{\mathrm{FH}}$ stabil. Allerdings erhalten wir, wie bereits angekündigt, keine Stabilität von $\Phi_{\bar{\gamma}}$ für festes $\bar{\gamma}$. Wir formulieren dies so:

Satz 4.9. Es gibt lineare Operatoren $D_{\mu}: \Phi_{\mathrm{FH}} \rightarrow \Phi_{\mathrm{FH}}$, die folgender Relation genügen:

$$
\sigma\left(D_{\mu} \phi\right)=\left.\frac{\partial}{\partial x^{\mu}} \sigma(\phi(x))\right|_{x=0} \quad \forall \phi \in \Phi_{\mathrm{FH}}, \sigma \in \bar{\Sigma} .
$$

Dabei gilt $D_{\mu} \Phi_{\bar{\gamma}} \subset \Phi_{\bar{\gamma}^{\prime}}$ mit $\bar{\gamma}^{\prime}=\max \{\bar{\gamma}+1,2 \bar{\gamma}\}$.

Das bedeutet, daß die Differentiation der Felder nicht aus dem berechneten Feldinhalt herausführt. Der Formalismus ist also sicher zur Formulierung von Kontinuitätsgleichungen und linearen Feldgleichungen geeignet; wir werden dies in Abschnitt 6.4 noch genauer ausführen.

Im Hinblick auf spätere Anwendungen betrachten wir auch Differentialoperatoren höherer Ordnung: Mit $\mathfrak{D}_{k}$ bezeichnen wir den von den Operatoren

$$
D_{\mu_{1}} \cdot \ldots \cdot D_{\mu_{n}}, \quad \mu_{j} \in\{0 \ldots s\}, 0 \leq n \leq k
$$

durch formale Linearkombinationen aufgespannten Vektorraum, wobei wir solche Operatoren identifizieren, die durch Permutation der Indizes auseinander hervorgehen. Durch den Fall $n=0$ ist oben der Einsoperator eingeschlossen. $\mathfrak{D}_{k}$ ist also der Raum der linearen Differentialoperatoren (höchstens) $k$-ter Ordnung. Durch mehrfache Anwendung von Satz 4.9 erhalten wir zu jedem $k$ und $\bar{\gamma}$ ein $\bar{\gamma}^{\prime}$, so daß

$$
\mathfrak{D}_{k} \Phi_{\bar{\gamma}} \subset \Phi_{\bar{\gamma}^{\prime}}
$$


Wir erwähnen kurz die Symmetrieeigenschaften der Differentialoperatoren: Durch die Festsetzung

$$
\alpha(\Lambda) D_{\mu}:=\Lambda_{\mu}^{\nu} D_{\nu}, \quad \alpha(\Lambda) 1:=1
$$

wirkt auf $\mathfrak{D}_{1}$ (und analog auf $\mathfrak{D}_{k}$ ) eine Darstellung der Lorentzgruppe. Man rechnet leicht nach, daß für $D \in \mathfrak{D}_{k}$ und $\phi \in \Phi_{\mathrm{FH}}$ gilt

$$
(\alpha(\Lambda) D)(\alpha(\Lambda) \phi)=\alpha(\Lambda) D \phi ;
$$

die Wirkung auf den Differentialoperatoren ist also kompatibel mit derjenigen auf $\Phi_{\mathrm{FH}}$.

Der Vollständigkeit halber zeigen wir noch, daß unsere Definition der Differentiation von Punktfeldern nach Ausintegrieren mit der üblichen Ableitung der WightmanDistributionen übereinstimmt. Dazu seien $\phi \in \Phi_{\mathrm{FH}}$ und $\sigma \in \Sigma(E), E$ fest. Wir integrieren das differenzierte Feld $D_{\mu} \phi$ aus:

$$
\begin{array}{rl}
\sigma\left(\left(D_{\mu} \phi\right)(f)\right)=\int d^{s+1} x & f(x) \sigma\left(U(x)\left(D_{\mu} \phi\right) U(x)^{*}\right) \\
=\left.\int d^{s+1} x f(x) \frac{\partial}{\partial y^{\mu}} \sigma\left(U(x)(U(y) \phi U(-y)) U(x)^{*}\right)\right|_{y=0} \\
=\int d^{s+1} x f(x) \frac{\partial}{\partial x^{\mu}} \sigma\left(U(x) \phi U(x)^{*}\right) \\
\stackrel{(\text { P.I.) }}{=}-\int d^{s+1} x \partial_{\mu} f(x) \sigma\left(U(x) \phi U(x)^{*}\right)=-\sigma\left(\phi\left(\partial_{\mu} f\right)\right) .
\end{array}
$$

Nach Fortsetzung erhält man somit

$$
\left(D_{\mu} \phi\right)(f)=-\phi\left(\partial_{\mu} f\right)
$$

als Operatorgleichung auf $\mathcal{C}^{\infty}(\mathcal{H})$. Damit sind auch die im Sinne von Distributionen differenzierten $\phi(f)$ in der Menge der „ausintegrierten“ Felder aus $\Phi_{\mathrm{FH}}$ enthalten.

\section{A Reguläre Projektionen in $\bar{\Sigma}^{*}$}

Es sei $V \subset \bar{\Sigma}^{*}$ ein Unterraum. Wir wollen Projektoren auf $V$ betrachten; dabei tritt das Problem auf, daß auf $\bar{\Sigma}^{*}$ a priori weder eine Hilbertraumstruktur noch eine Topologie definiert sind, die uns eine sinnvolle Analyse von Projektoren erlauben würden. Ist aber $V$ endlichdimensional, so kann man diesen Raum in natürlicher Weise mit der Standardtopologie versehen. Die Stetigkeit des Projektors fordern wir durch folgendes Axiom:

Definition 4.10. Sei $V \subset \bar{\Sigma}^{*}$ ein endlichdimensionaler Unterraum. Eine reguläre Projektion auf $V$ ist eine lineare Abbildung $p: \bar{\Sigma}^{*} \rightarrow \bar{\Sigma}^{*}$, für die gilt

$$
p^{2}=p, \quad \text { Bild } p=V,
$$

und für die zu jedem $v^{*} \in V^{*}$ ein $E>0$ existiert, so daß

$$
v^{*} \circ p \in \Sigma(E) \text {. }
$$


Aus der Definition folgt direkt, daß $p\left\lceil V=\mathrm{id}_{V}\right.$, wie man es von einer Projektion auch erwartet.

Ist eine reguläre Projektion $p$ gegeben, so können wir durch Wahl einer Basis $\left\{v_{j}\right\}$ von $V$ offenbar eine Summendarstellung der folgenden Form finden:

$$
p=\sum_{j=1}^{\operatorname{dim} V} \sigma_{j}(\cdot) v_{j} \quad \text { mit } \sigma_{j} \in \Sigma(E), E>0 \text { geeignet. }
$$

Ist andererseits $p$ eine Abbildung von der Form (4.A.1) und gilt $p^{2}=p$, dann ist $p$ offenbar eine reguläre Projektion auf $V:=\operatorname{Span}\left\{v_{j}\right\}$, wenn man noch voraussetzt, daß die $\sigma_{j}$ linear unabhängig sind. Insofern lassen sich die regulären Projektionen leicht charakterisieren.

Wir zeigen nun, daß solche Projektionen immer existieren. Dazu sei ein $V \subset \bar{\Sigma}^{*}$ mit endlicher Dimension $n$ fest gegeben. Wir wählen eine Basis $\left\{v_{j}\right\}$ von $V$. Dann betrachten wir die Abbildung

$$
\chi: \bigcup_{E} \Sigma(E) \rightarrow \mathbb{C}^{n}, \quad \sigma \mapsto\left(\sigma\left(v_{j}\right)\right)_{j=1}^{n}
$$

$\chi$ ist surjektiv, denn sonst wären die $v_{j}$ nicht linear unabhängig. Wir wählen Urbilder $\sigma_{j}$ der Standard-Basisvektoren des $\mathbb{C}^{n}$; dann gilt also $\sigma_{i}\left(v_{j}\right)=\delta_{i j}$. Die Abbildung $p:=\sum_{j} \sigma_{j}(\cdot) v_{j}$ ist dann von der Form (4.A.1) und erfüllt $p^{2}=p$, ist also eine reguläre Projektion. Damit haben wir bewiesen:

Satz 4.11. Sei $V \subset \bar{\Sigma}^{*}$ ein endlichdimensionaler Unterraum. Dann existiert eine reguläre Projektion auf $V$.

Wir bemerken noch, daß sich reguläre Projektionen wegen der geforderten Stetigkeitseigenschaften leicht auch als „präduale Abbildungen“ auf $\bar{\Sigma}$ übertragen lassen: Wir definieren $p_{*}: \bar{\Sigma} \rightarrow \bar{\Sigma}$ durch

$$
p_{*} \sigma(\cdot):=\sigma(p \cdot)=\sum_{j} \sigma\left(v_{j}\right) \sigma_{j}
$$

was offenbar energiebeschränkte schwach stetige Funktionale wieder in solche überführt (wobei sich die Energieschranke evtl. ändert). 


\section{Teil II}

\section{Produkte von Feldern}





\section{Kapitel 5}

\section{Produktentwicklungen}

Zur Untersuchung von Wechselwirkungstermen und Korrelationsfunktionen in der Quantenfeldtheorie benötigt man eine Produktbildung zwischen den betrachteten Punktfeldern. Dabei ist bekannt, daß das Produkt von Punktfeldern am selben Raum-Zeit-Punkt im allgemeinen nicht wohldefiniert ist, sondern zu Divergenzen führt. Um diese zu analysieren, verwendet man sogenannte Operatorproduktentwicklungen [Wil69], das sind Reihenentwicklungen des Produkts von Feldern an verschiedenen Raum-Zeit-Punkten, die man im Limes kleiner Abstände untersucht.

Solche Produktentwicklungen wurden im axiomatischen Kontext [W/72, SSe73] wie auch in störungstheoretischen und exakt lösbaren Modellen [Zim70, Wil70a, Wil70b, Low70] betrachtet; sie entwickelten sich im Lagrange'schen Zugang zur Quantenfeldtheorie zu einem wichtigen Hilfsmittel (z.B. [CG73, BDLW75]; siehe auch [Wei96, ch. 20]). Darüberhinaus sind sie im speziellen Rahmen der konformen Feldtheorie genau bekannt [SSV75, Lüs76], Mac77]. Unser Ziel ist es, unter Verwendung der besprochenen Phasenraumeigenschaften eine mathematisch rigorose, modellübergreifende Form der Operatorproduktentwicklung zwischen den konstruierten Punktfeldern herzuleiten.

Die Produkte der Punktfelder lassen sich in unserem Kontext als Grenzwerte der Produkte der approximierenden lokalen Operatoren einführen. Wir zeigen zunächst, daß dies aufgrund der Lokalitätseigenschaften und Energieschranken - tatsächlich ein wohldefiniertes Produkt von Feldern an raumartig getrennten Punkten ergibt. Unter Verwendung der Phasenraum-Approximation können wir dann hierfür eine Operatorproduktentwicklung im Sinne einer asymptotischen Reihe angeben.

Wir diskutieren die Symmetrieeigenschaften dieser Entwicklung, die vor allem im Zusammenhang mit spontan gebrochenen Symmetrien von Interesse sind.

Anschließend geben wir noch eine analoge Produktentwicklung für Felder mit beliebigen (nicht notwendig raumartigen) Abständen an; hier lassen sich Produkte wie Produktentwicklung aber im allgemeinen nur im Sinne von Distributionen interpretieren. 


\subsection{Problemstellung}

Die Einführung einer Produktstruktur zwischen punktartig lokalisierten Quantenfeldern $\phi(x)$ am selben Raum-Zeit-Punkt, wie sie in Wechselwirkungstermen wie $\phi(x)^{4}$ verwendet wird, bereitet grundsätzliche Probleme. Wie wir in unserer Diskussion gesehen hatten, existieren die $\phi(x)$ im allgemeinen nicht als Operatoren, sondern nur als quadratische Formen, so daß ein Produkt zwischen ihnen nicht in natürlicher Weise definiert werden kann. Führt man das Produkt als Multiplikation der ,ausgeschmierten“ Wightman-Felder ein, etwa $\phi(f) \phi^{\prime}(g)$ für zwei Punktfelder $\phi, \phi^{\prime}$, dann kann man im allgemeinen nicht mehr im Sinne eines Grenzübergangs zu einem Punktfeld bzw. zu einer Wightman-Distribution (in einer Minkowskiraum-Variable) übergehen; schon in der freien Feldtheorie wird man hierbei auf Divergenzen geführt.

Zur Analyse dieser Singularitäten bei Produkten am gleichen Punkt betrachtet man den Ausdruck $\phi(x) \phi^{\prime}(y)$, der entweder für bestimmte (z.B. raumartige) Abstände oder im Sinne von Distributionen definiert werden kann, im Limes $y \rightarrow x$. Wie Wilson [Wil69] vermutete, sollte sich dieser Ausdruck in eine Reihe von Punktfeldern entwickeln lassen, die z.B. am Punkt $\frac{x+y}{2}$ lokalisiert sind:

$$
\phi(x) \phi^{\prime}(y)=\sum_{j} c_{j}(x-y) \phi_{j}\left(\frac{x+y}{2}\right) .
$$

Die $c_{j}(x-y)$ sind dabei „c-Zahl-Funktionen“, genauer Distributionen, die typischerweise auf dem Lichtkegel Singularitäten aufweisen [OZ72].

Solche sogenannten Operatorproduktentwicklungen wurden axiomatisch im Rahmen der Wightman-Theorie von Wilson und Zimmermann [W/72] untersucht. Die Existenz der Entwicklung konnte dabei nicht auf die Wightman-Axiome zurückgeführt werden, sondern die Autoren formulieren zusätzliche Annahmen an die Theorie, die die Existenz der Produktentwicklung sichern, aber mehr „ad hoc" zu sein scheinen.

Aus diesen Annahmen heraus konnten Otterson und Zimmermann für ein Produkt zweier Felder eine sehr detaillierte Klassifikation der $x$-Abhängigkeit der Koeffizientenfunktionen $c_{j}(x)$ ableiten [0772]; allerdings hängen diese Ergebnisse sehr kritisch davon $\mathrm{ab}$, daß die Funktionen sich in einer faktorisierten Form $c_{j}(x)=\left(x^{2}\right)^{\lambda} \hat{c}_{j}\left(\frac{x}{\sqrt{-x^{2}}}\right)$ schreiben lassen, was bei Wilson und Zimmermann eher eine Annahme als eine Folgerung ist.

Schlieder und Seiler [SSe73] gelang es, die Existenz der Operatorproduktentwicklung auf die Singularitätenstruktur der 4-Punkt-Funktion zurückzuführen, was in einigen speziellen Fällen eine genauere Analyse der Entwicklungsterme erlaubt.

Sehr viel genauer ist die Kenntnis über Produktentwicklungen im speziellen Rahmen der konformen Feldtheorie [SSV75, Lüs76, Mac77]. Hier existiert die Produktentwicklung stets, wenn man annimmt, daß die Theorie „genügend viele“ lokale Punktfelder enthält. Solch einen vollständigen Satz von Punktfeldern liefert z.B. die Analyse von Fredenhagen und Jörß [F.J96]. Allerdings verwenden diese Ergebnisse sehr explizit die konforme Symmetrie, zum Teil auch spezielle Eigenschaften der 1+1-dimensionalen Theorie, so daß sie sich kaum auf allgemeinere Modelle übertragen lassen.

Wir stellen hier eine Operatorproduktentwicklung vor, die aus dem asymptotischen Phasenraumkriterium folgt. In unserem Kontext sind die Produkte der Felder durch die algebraische Struktur der $\mathfrak{A}(r)$ bereits bestimmt: Die Punktfelder $\phi \in \Phi_{\mathrm{FH}}$ lassen sich 
durch Folgen $A_{r} \in \mathfrak{A}(r), r \rightarrow 0$, approximieren, und das Produkt der Felder wird man als Limes der Produktfolge definieren, falls dieser existiert. (Wir werden genauer klären, inwieweit dies der Fall ist.) Auf ein Produkt

$$
A_{r}^{(0)}\left(x_{0}\right) \cdot \ldots \cdot A_{r}^{(n)}\left(x_{n}\right) \stackrel{r \rightarrow 0}{\longrightarrow} \phi^{(0)}\left(x_{0}\right) \cdot \ldots \cdot \phi^{(n)}\left(x_{n}\right)
$$

kann man dann die Phasenraum-Approximation $\left(\Xi \approx p_{\bar{\gamma}} \Xi\right)$ anwenden, um so eine Operatorproduktentwicklung nach Punktfeldern $\phi_{j} \in \Phi_{\bar{\gamma}}$ zu erhalten. Diese Entwicklung folgt also aus den allgemeinen Axiomen der algebraischen Feldtheorie zusammen mit zusätzlichen Phasenraumeigenschaften.

\subsection{Raumartige Produkte}

Wie bereits erwähnt, sind für Punktfelder $\phi^{(0)}, \ldots, \phi^{(n)}$ die Produkte

$$
\phi^{(0)}\left(x_{0}\right) \cdot \ldots \cdot \phi^{(n)}\left(x_{n}\right)
$$

im allgemeinen nicht erklärt. Wir werden jedoch in diesem Abschnitt zeigen, daß für RaumZeit-Punkte $x_{0}, \ldots, x_{n}$, die zueinander paarweise raumartig getrennt liegen, solch ein Produkt in eindeutiger Weise definiert werden kann. Dies folgt aus den Lokalitätseigenschaften der Felder sowie ihren polynomialen Energieschranken; das asymptotische Phasenraumkriterium spielt dabei keine direkte Rolle.

Wir gehen aus von einem Satz $\phi^{(0)}, \ldots, \phi^{(n)} \in \Phi_{\bar{\gamma}}$ von Punktfeldern, wobei $\bar{\gamma}$ im folgenden festgehalten wird. Nach Satz 3.17 können wir uns ein $l>0$ und $n+1$ Folgen lokaler Operatoren $A_{r}^{(j)} \in \mathfrak{A}(r)$ verschaffen, die die lokalen Felder $\phi^{(j)}$ approximieren:

$$
\left\|R^{l}\left(A_{r}^{(j)}-\phi^{(j)}\right) R^{l}\right\| \leq r \cdot \text { const. } \quad \forall j \in\{0, \ldots, n\} .
$$

(Hierbei hängt $l$ nur von $\bar{\gamma}$ ab.) Das Produkt der Punktfelder soll definiert werden als

$$
\phi^{(0)}\left(x_{0}\right) \cdot \ldots \cdot \phi^{(n)}\left(x_{n}\right):=\lim _{r \rightarrow 0} A_{r}^{(0)}\left(x_{0}\right) \cdot \ldots \cdot A_{r}^{(n)}\left(x_{n}\right),
$$

wobei wir $A(x)=U(x) A U(x)^{*}$ usw. schreiben. Wir interessieren uns für den Fall, daß die $x_{j}$ raumartig getrennt sind, das heißt formaler, daß der Vektor $x=\left(x_{0}, \ldots, x_{n}\right)$ in der Menge

$$
\mathcal{M}_{\mathrm{R}}^{n+1}:=\left\{x \in \mathcal{M}^{n+1} \mid\left(x_{i}-x_{j}\right)^{2}<0 \forall i, j \in\{0, \ldots, n\}, i \neq j\right\}
$$

liegt. Unter dieser Voraussetzung zeigen wir, daß der Grenzübergang (5.2.3) tatsächlich eine Linearform auf $\bar{\Sigma}$ definiert, wobei der Grenzwert unabhängig von den approximierenden Folgen ist - er hat insofern als Produkt der Punktfelder intrinsische Bedeutung. Für die spätere Etablierung einer Operatorproduktentwicklung ist dabei nicht nur die Tatsache der Konvergenz interessant, sondern wir benötigen auch detaillierte Abschätzungen über die Konvergenzgeschwindigkeit in Abhängigkeit von $r$ und vom Abstand des Punktes $x$ zum Rand des „Definitionsbereichs“ $\mathcal{M}_{\mathrm{R}}^{n+1}$, den wir als dist $\left(x, \partial \mathcal{M}_{\mathrm{R}}^{n+1}\right)$ notieren.

Zum Erhalt dieser Ergebnisse verwenden wir funktionentheoretische Methoden, indem wir die Matrixelemente der quadratischen Formen in (5.2.3) als Grenzwerte holomorpher Funktionen darstellen. Als wesentlich erweisen sich hier das Maximumprinzip und 
das Edge-Of-The-Wedge-Theorem; diese Analysemethoden sind in Anhang 5.A näher ausgeführt.

Um zum funktionentheoretischen Kontext überzugehen, wählen wir zunächst ein Vektorfunktional $\sigma=\left(\xi|\cdot| \xi^{\prime}\right) \in \Sigma(E)$ fest (mit gewissem $E$ ); der Einfachheit halber sei $\sigma$ normiert. Wir definieren dann die Funktionen

$$
f_{r}\left(z_{1}, \ldots, z_{n}\right):=\sigma\left(U\left(x_{0}\right) A_{r}^{(0)} U\left(z_{1}\right) A_{r}^{(1)} \ldots U\left(z_{n}\right) A_{r}^{(n)} U\left(-x_{0}-z_{1}-\ldots-z_{n}\right)\right)
$$

zunächst für reelle $z=\left(z_{1}, \ldots, z_{n}\right) \in \mathcal{M}^{n} . x_{0} \in \mathcal{M}$ ist dabei festgehalten. Die Funktionen $f_{r}$ sind stetig und lassen sich als stetige Randwerte von Funktionen darstellen, die auf $\left(\mathcal{M}+i V_{+}\right)^{n}$ holomorph sind: Man erweitert $U\left(z_{j}\right) \mathrm{zu} e^{i P_{\mu} z_{j}^{\mu}}$, wobei die Potenzreihe der Exponentialfunktion aufgrund der Spektrumsbedingung für $\operatorname{Im} z_{j} \in V_{+}$absolut konvergiert. Der Faktor $e^{-i P_{\mu} \operatorname{Im}\left(z_{1}+\ldots+z_{n}\right)^{\mu}}$ rechts im Argument des Funktionals stört dabei nicht, da $\sigma$ energiebeschränkt ist. Die so erhaltenen holomorphen Funktionen, die wir ebenfalls mit $f_{r}$ bezeichnen, ergeben wegen der starken Stetigkeit der Translationen im Limes $\operatorname{Im} z_{j} \rightarrow 0$ wieder den Ausdruck (5.2.5). Man hat für sie folgende Schranken:

$$
\begin{aligned}
&\left|f_{r}(z)\right| \leq\left\|P(E) R^{-l}\right\| \cdot\left\|R^{l} A_{r}^{(0)} R^{l}\right\| \cdot\left\|R^{-l} e^{i P_{\mu} z_{1}^{\mu}} R^{-l}\right\| \cdot\left\|R^{l} A_{r}^{(1)} R^{l}\right\| \cdot \ldots \\
& \ldots \cdot\left\|R^{-l} e^{i P_{\mu} z_{n}^{\mu}} R^{-l}\right\| \cdot\left\|R^{l} A_{r}^{(n)} R^{l}\right\| \cdot\left\|R^{-l} P(E)\right\| \cdot\left\|P(E) e^{-i P_{\mu} \sum z_{j}^{\mu}}\right\| \\
& \leq\left(\frac{1}{1+E}\right)^{2 l}\left(\prod_{j=0}^{n}\left\|R^{l} A_{r}^{(j)} R^{l}\right\|\right)\left(\prod_{j=1}^{n}\left\|R^{-2 l} e^{-P_{\mu} \operatorname{Im} z_{j}^{\mu}}\right\|\right)\left(\prod_{j=1}^{n}\left\|P(E) e^{+P_{\mu} \operatorname{Im} z_{j}^{\mu}}\right\|\right) .
\end{aligned}
$$

Dabei sind die $R^{l} A_{r} R^{l}$ normkonvergente Folgen, so daß die Folge ihrer Normen im Limes $r \rightarrow 0$ beschränkt bleibt.

Für $y \in V_{+}$ist weiter $P_{(y)}:=y^{\mu} P_{\mu}$ ein positiver Operator, der nicht nur mit $H$ kommutiert, sondern sich auch abschätzen läßt als $c_{1} H \leq P_{(y)} \leq c_{2} H$ mit positiven Konstanten $c_{1}$ und $c_{2}$, die lokal gleichmäßig in $y$ gewählt werden können. Wir wählen einen offenen konvexen Teilkegel $\mathcal{C}$, dessen Abschluß $\overline{\mathcal{C}}$ ganz in $V_{+}$liegt; dann können aus Kompaktheitsgründen die $c_{1}, c_{2}$ für alle normierten $y \in \overline{\mathcal{C}}$ gleich gewählt werden. Halten wir nun ein $y \in \mathcal{C}$ fest und betrachten $z \in \mathbb{C}^{s+1}$ mit $\operatorname{Im} z=y$, dann haben wir

$$
\begin{array}{r}
\left\|R^{-2 l} e^{-P_{\mu} \operatorname{Im} z^{\mu}}\right\|=\left\|R^{-2 l} e^{-P_{(y)}}\right\| \leq\left\|(1+H)^{2 l} e^{-c_{1}\|y\| H}\right\|=\sup _{\lambda \geq 0}\left((1+\lambda)^{2 l} e^{-c_{1}\|y\| \lambda}\right) \\
\underset{\left(\lambda^{\prime}=\lambda\|y\|\right)}{\leq}\|y\|^{-2 l} \sup _{\lambda^{\prime} \geq 0}\left(\left(\|y\|+\lambda^{\prime}\right)^{2 l} e^{-c_{1} \lambda^{\prime}}\right) \leq\|y\|^{-2 l} \cdot c_{3} \quad \text { etwa für }\|y\| \leq 1,
\end{array}
$$

wobei die Konstante $c_{3}$ nicht von $y$ abhängt. Ganz ähnlich ergibt sich

$$
\left\|P(E) e^{+P_{\mu} \operatorname{Im} z^{\mu}}\right\|=\left\|P(E) e^{P_{(y)}}\right\| \leq\left\|P(E) e^{+c_{2}\|y\| H}\right\| \leq e^{c_{2} E} \quad(\|y\| \leq 1) .
$$

Fassen wir diese Ergebnisse in der Abschätzung (5.2.6) zusammen, so ergibt sich für $\operatorname{Im} z=$ $y \in \mathcal{C}^{n},\|y\| \leq 1$ folgende Schranke für $f_{r}(z)$ :

$$
\left|f_{r}(z)\right| \leq c_{4}(E) \cdot\|y\|^{-2 n l} .
$$

Per Konstruktion ist die Konstante $c_{4}(E)$ dabei

- unabhängig von $\sigma$ (solange $E$ fest ist und $\|\sigma\|=1$ ) sowie von $x_{0}$, 
- unabhängig von $r$ und von $z$, soweit $\operatorname{Im} z \in \mathcal{C}^{n}$.

Insbesondere ist die Familie der $f_{r}$ auf $(\mathcal{M}+i \mathcal{C})^{n}$ lokal gleichmäßig beschränkt.

Neben den $f_{r}$ betrachten wir nun auch die „permutierten Matrixelemente" $\hat{f}_{r}$ :

$$
\hat{f}_{r}\left(z_{1}, \ldots, z_{n}\right):=\sigma\left(U\left(x_{0}+z_{1}+\ldots+z_{n}\right) A_{r}^{(n)} U\left(-z_{n}\right) \ldots A_{r}^{(1)} U\left(-z_{1}\right) A_{r}^{(0)} U\left(-x_{0}\right)\right),
$$

zunächst wieder für reelle $z_{1}, \ldots, z_{n}$; die Funktionen lassen sich aber ganz analog wie oben als Randwerte holomorpher Funktionen darstellen, wobei das Holomorphiegebiet diesmal $\left(\mathcal{M}-i V_{+}\right)^{n}$ umfaßt. Die $\hat{f}_{r}(z)$ genügen entsprechenden Schranken, wie sie in (5.2.9) schon für $f_{r}(z)$ berechnet wurden.

Der Grund für diese Definition ist folgender: Wir betrachten den Fall, daß die Punkte $x_{j}$ paarweise raumartig getrennt sind. (Bei festgehaltenem $x_{0}$ lassen sich $x_{1}, \ldots, x_{n}$ und die reellen Punkte $z_{1}, \ldots, z_{n}$ eindeutig ineinander umrechnen.) Wegen der Lokalität der $A_{r}^{(j)}$ vertauschen dann die translatierten Operatoren $A_{r}^{(j)}\left(x_{j}\right)$ im Argument von $\sigma$ für kleine $r$ (siehe die Definitionen (5.2.5) und (5.2.10)); folglich stimmen $f_{r}(z)$ und $\hat{f}_{r}(z)$ an diesen reellen Punkten überein.

Formaler muß dazu $x \in \mathcal{M}_{\mathrm{R}}^{n+1}$ sein, was für $z$ bedeutet, daß es in der Menge aus reellen Punkten

$$
\mathcal{M}_{\mathrm{DR}}^{n}:=\left\{z \in \mathcal{M}^{n} \mid\left(\sum_{j=j_{0}}^{j_{1}} z_{j}\right)^{2}<0 \forall j_{0} \leq j_{1}\right\}
$$

liegen muß. Wir wissen also, daß $f_{r}(z)=\hat{f}_{r}(z)$ in einer reellen Umgebung jedes festgehaltenen Punkts $\tilde{z} \in \mathcal{M}_{\mathrm{DR}}^{n}$, wenn man $r$ zu $\tilde{z}$ geeignet klein wählt.

Diese Ergebnisse erlauben uns, das Edge-of-the-Wedge-Theorem auf $f_{r}, \hat{f}_{r}$ anzuwenden. Zunächst sollen jedoch Abschätzungen für diese Funktionen in der Nähe der reellen Punkte etabliert werden.

Wir betrachten dazu zunächst ein festes $y \in \mathcal{C}^{n}$ mit $\|y\|=1$. Es sei $\tilde{z} \in \mathcal{M}_{\mathrm{DR}}^{n}$ (also reell). Die Funktionen

$$
f_{r, y}(t):= \begin{cases}f_{r}(\tilde{z}+t y) & \text { für } \operatorname{Im} t>0 \\ \hat{f}_{r}(\tilde{z}+t y) & \text { für } \operatorname{Im} t<0\end{cases}
$$

sind holomorph in $\mathbb{C} \backslash \mathbb{R}$ und (für kleine $r$ ) nach obigen Ergebnissen stetig fortsetzbar auf eine gewisse reelle Nullumgebung, deren Größe nur von $\tilde{z}$ und $r$ abhängt. Mit der Abkürzung士

$$
d(\tilde{z}):=\min \left\{1, \operatorname{dist}\left(\tilde{z}, \partial \mathcal{M}_{\mathrm{DR}}^{n}\right)\right\} \quad\left(\tilde{z} \in \mathcal{M}_{\mathrm{DR}}^{n}\right)
$$

ist diese Nullumgebung z.B. gegeben durch $|t|<\frac{1}{2} d(\tilde{z})$, wenn $r<\frac{1}{4} d(\tilde{z})$. Nach dem „eindimensionalen Edge-of-the-Wedge-Theorem" (Satz 5.8 in Anhang 5.A.2) ist $f_{r, y}$ dann tatsächlich holomorph auf $\mathbb{C} \backslash\left\{t|\operatorname{Im} t=0| t \mid, \geq \frac{1}{2} d(\tilde{z})\right\}$. Die Funktion erfüllt nach (5.2.9) die Schranken

$$
\left|f_{r, y}(t)\right| \leq c_{4}(E)|\operatorname{Im} t|^{-2 n l} \quad \text { für }|t|<\frac{1}{2} d(\tilde{z})
$$

\footnotetext{
${ }^{1}$ Die Definition wird zur Vereinfachung der späteren Abschätzungen so gewählt, daß stets $d(\tilde{z}) \leq 1$.
} 
Ein Phragmén-Lindelöf-Argument liefert nun die Abschätzung

$$
\left|f_{r, y}(t)\right| \leq c_{5}(E) d(\tilde{z})^{-2 n l} \quad \text { für }|t|<\frac{d(\tilde{z})}{4},
$$

wobei wieder $c_{5}(E)$ nicht von $y$ abhängt. Schreiben wir dies als Ungleichung für die Funktion $f_{r}$, indem wir $t$ rein imaginär wählen, so erhalten wir

$$
\left|f_{r}(\tilde{z}+i y)\right| \leq c_{5}(E) d(\tilde{z})^{-2 n l} \quad \text { für } \tilde{z} \in \mathcal{M}_{\mathrm{DR}}^{n}, y \in \mathcal{C}^{n},\|y\|<\frac{d(\tilde{z})}{4}, r<\frac{d(\tilde{z})}{4} .
$$

Insbesondere gilt diese Abschätzung bei $y=0$, d.h. an den reellen Punkten.

Nun setzen wir die Funktionen $f_{r}$ und $\hat{f}_{r}$ selbst per Edge-of-the-Wedge-Theorem fort (Satz 5.9 in Anhang 5.A.2); als Kegel verwenden wir das oben eingeführte $\mathcal{C}^{n}$, dann existieren die Grenzwerte der Funktionen für $\operatorname{Im} z \rightarrow 0$ in der gewünschten Weise. Die holomorph fortgesetzten Funktionen bezeichnen wir wieder mit $f_{r}$. Diese Funktionen $f_{r}$ sind holomorph auf $(\mathcal{M} \pm i \mathcal{C})^{n}$ und einer komplexen Umgebung eines Teilgebiets von $\mathcal{M}_{\mathrm{DR}}^{n}$, wobei dieses Teilgebiet für kleine $r$ jeden festgehaltenen Punkt von $\mathcal{M}_{\mathrm{DR}}^{n}$ umfaßt. Konkret enthält das Holomorphiegebiet eine komplexe Kugel vom Radius $r_{0} \cdot d(\tilde{z})$ um $\tilde{z}$ für jedes $\tilde{z} \in \mathcal{M}_{\mathrm{DR}}^{n}$; $r_{0}$ ist eine durch $\mathcal{C}$ festgelegte Konstante (siehe Korollar 5.10). Die Abschätzungen (5.2.16) lassen sich nach demselben Korollar wie folgt auf die fortgesetzten Funktionen übertragen:[3

$$
\left|f_{r}(z)\right| \leq c_{5}(E) d(\tilde{z})^{-2 n l} \quad \text { für } \quad \tilde{z} \in \mathcal{M}_{\mathrm{DR}}^{n},|z-\tilde{z}|<\frac{r_{0}}{4 r_{1}} \cdot d(\tilde{z}) \text { und kleine } r .
$$

Hier ist $r_{1}$ eine weitere (durch $\mathcal{C}$ festgelegte) Konstante.

Wir untersuchen nun die Konvergenz der $f_{r}$ für $r \rightarrow 0$. Zunächst zeigen wir, daß sie auf $\left(\mathcal{M}+i V_{+}\right)^{n}$ punktweise gegen eine holomorphe Funktion konvergieren. Es sei dazu für $z \in\left(\mathcal{M}+i V_{+}\right)^{n}$ :

$$
f(z):=\sigma\left(U\left(x_{0}\right) \phi^{(0)} U\left(z_{1}\right) \ldots U\left(z_{n}\right) \phi^{(n)} U\left(-x_{0}-z_{1}-\ldots-z_{n}\right)\right) .
$$

Das ist wohldefiniert, da $\left\|R^{-l} U\left(z_{j}\right) R^{-l}\right\|<\infty$ usw., es ist also „,genügend Energiedämpfung vorhanden". $f(z)$ ist im angegebenen Bereich sogar holomorph (via Entwicklung der $U\left(z_{k}\right)$ in Potenzreihen). Um den angesprochenen Grenzübergang zu etablieren, schreibt man nun

$$
\begin{aligned}
& f(z)-f_{r}(z)= \\
& \quad \sigma\left(U\left(x_{0}\right)\left(\phi^{(0)}-A_{r}^{(0)}\right) U\left(z_{1}\right) \phi^{(1)} U\left(z_{2}\right) \ldots \phi^{(n)} U\left(-x_{0}-z_{1}-\ldots-z_{n}\right)\right) \\
& +\sigma\left(U\left(x_{0}\right) A_{r}^{(0)} U\left(z_{1}\right)\left(\phi^{(1)}-A_{r}^{(1)}\right) U\left(z_{2}\right) \ldots \phi^{(n)} U\left(-x_{0}-z_{1}-\ldots-z_{n}\right)\right) \\
& +\ldots \\
& +\sigma\left(U\left(x_{0}\right) A_{r}^{(0)} U\left(z_{1}\right) A_{r}^{(1)} U\left(z_{2}\right) \ldots\left(\phi^{(n)}-A_{r}^{(n)}\right) U\left(-x_{0}-z_{1}-\ldots-z_{n}\right)\right)
\end{aligned}
$$

2 Siehe Anhang 5.A.1. Zur Anwendung des Arguments beschreibt man dem betrachteten Gebiet ein geeignetes Quadrat ein.

${ }^{3}$ Wir wählen dazu in Korollar 5.10 die Funktion $g(\|y\|)$ konstant für $\|y\|<\frac{1}{4} d(\tilde{z})$. 
In dieser Summe schätzen wir wie folgt ab (wobei $\operatorname{Im} z=t \cdot y$ mit $0<t<1$ und $y \in \mathcal{C}^{n}$, $\|y\|=1)$ :

$$
\begin{aligned}
& \left\|R^{l}\left(\phi^{(j)}-A_{r}^{(j)}\right) R^{l}\right\| \leq r \cdot \text { const.; } \\
& \left\|R^{l} \phi^{(j)} R^{l}\right\| \leq \text { const. }, \quad\left\|R^{l} A_{r}^{(j)} R^{l}\right\| \leq \text { const. } \\
& \left\|R^{-l} U\left(z_{j}\right) R^{-l}\right\| \leq t^{-2 l} c_{3}, \\
& \left\|R^{-l} P(E)\right\| \leq\left(\frac{1}{1+E}\right)^{l}, \quad\left\|P(E) U\left(-x_{0}-z_{1}-\ldots-z_{n}\right)\right\| \leq e^{t c_{2} E},
\end{aligned}
$$

wobei $c_{2}, c_{3}$ von oben übernommen sind. Damit erhält man

$$
\left|f(z)-f_{r}(z)\right| \leq c_{6} \cdot\left(\frac{1}{1+E}\right)^{2 l} e^{t c_{2} E} \cdot r \cdot t^{-2 l n} .
$$

Insbesondere gilt (da sich entsprechende Abschätzungen auch für $t \geq 1$ finden lassen):

$$
\lim _{r \rightarrow 0} f_{r}(z)=f(z) \quad \forall z \in(\mathcal{M}+i \mathcal{C})^{n}
$$

Entsprechend erhält man, daß die $\hat{f}_{r}$ auf dem Gebiet $(\mathcal{M}-i \mathcal{C})^{n}$ punktweise gegen ein entsprechend definiertes holomorphes $\hat{f}$ konvergieren. Diese Grenzwerte sind offenbar unabhängig von den approximierenden Folgen $A_{r}^{(j)}$ definiert.

Wir hatten bereits gezeigt, daß die $f_{r}$ eine beschränkte Familie holomorpher Funktionen bilden. Konvergiert eine Folge daraus punktweise auf einer offenen Menge, so muß sie auf ihrem gesamten Holomorphiegebiet lokal gleichmäßig gegen eine holomorphe Grenzfunktion konvergieren. Dies gilt dann auch für die fortgesetzten Funktionen $f_{r}$; wir können also auch die Grenzfunktion $f$ nach $(\mathcal{M} \pm i \mathcal{C})^{n}$ sowie in eine komplexe Umgebung von $\mathcal{M}_{\mathrm{DR}}^{n}$ fortsetzen. Insbesondere erhalten wir an den reellen Punkten:

$$
f\left(z_{1}, \ldots, z_{n}\right)=\lim _{r \rightarrow 0} f_{r}\left(z_{1}, \ldots, z_{n}\right), \quad z \in \mathcal{M}_{\mathrm{DR}}^{n}
$$

Wir schreibent

$$
\begin{aligned}
\sigma\left(\phi^{(0)}\left(x_{0}\right) \cdot \ldots \cdot \phi^{(n)}\left(x_{n}\right)\right):=f\left(x_{1}-x_{0}, \ldots, x_{n}-x_{n-1}\right) & \\
& \text { für }\left(x_{0}, \ldots, x_{n}\right) \in \mathcal{M}_{\mathrm{R}}^{n+1}
\end{aligned}
$$

und haben damit die raumartigen Operatorprodukte im Sinne quadratischer Formen definiert. Es ist klar, daß diese Definition linear in $\sigma$ ist; alle Abschätzungen sind zudem gleichmäßig in normiertem $\sigma$ (bei festem $E$ ), also ist tatsächlich $\phi^{(0)}\left(x_{0}\right) \cdot \ldots \cdot \phi^{(n)}\left(x_{n}\right) \in \bar{\Sigma}^{*}$ wohldefiniert.

Durch erneute Anwendung des Phragmén-Lindelöf-Arguments aus Anhang 5.A.1 können wir auch für den Grenzprozeß noch Abschätzungen an den reellen Punkten etablieren: Aus (5.2.21) folgt

$$
\left|f(z)-f_{r}(z)\right| \leq c_{7}(E) \cdot r \cdot d(z)^{-2 n l} \quad \text { für } z \in \mathcal{M}_{\mathrm{DR}}^{n}, r<\frac{1}{4} d(z)
$$

\footnotetext{
${ }^{4}$ Man beachte, daß die Definition der Funktion $f$ von $\sigma$ und $x_{0}$ als „Parameter" abhängig ist.
} 
respektive für die Linearformen

$$
\begin{aligned}
\left\|\phi^{(0)}\left(x_{0}\right) \cdot \ldots \cdot \phi^{(n)}\left(x_{n}\right)-A_{r}^{(0)}\left(x_{0}\right) \cdot \ldots \cdot A_{r}^{(n)}\left(x_{n}\right)\right\|_{E} & \leq c_{7}(E) \cdot r \cdot \hat{d}(x)^{-2 n l} \\
& \quad \text { für } x \in \mathcal{M}_{\mathrm{R}}^{n+1}, r<\frac{1}{4} \hat{d}(x) .
\end{aligned}
$$

Hierbei ist

$$
\hat{d}(x):=\min \left\{1, \operatorname{dist}\left(x, \partial \mathcal{M}_{\mathrm{R}}^{n+1}\right)\right\}
$$

gesetzt, und man bemerkt, daß mit der verwendeten Relation zwischen $z$ und $x$ stets gilt: $d(z)=\hat{d}(x)$.

Aufgrund der Schranken an das Produkt der lokalen Operatoren aus (5.2.16) erhält man außerdem zusammen mit (5.2.25), daß

$$
\left\|\phi^{(0)}\left(x_{0}\right) \cdot \ldots \cdot \phi^{(n)}\left(x_{n}\right)\right\|_{E} \leq c_{8}(E) \cdot \hat{d}(x)^{-2 n l} \quad \text { für } x \in \mathcal{M}_{\mathrm{R}}^{n+1} \text {. }
$$

Wir fassen die in diesem Abschnitt gewonnenen Aussagen über die Existenz raumartiger Operatorprodukte nun in einem Satz zusammen.

Satz 5.1 (Existenz raumartiger Operatorprodukte). Sei $\bar{\gamma}>0$ fest, und seien $\phi^{(0)}, \ldots, \phi^{(n)} \in \Phi_{\bar{\gamma}}$ lokale Punktfelder. Dann existiert zu jedem $x \in \mathcal{M}_{\mathrm{R}}^{n+1}$ eine Linearform

$$
\phi^{(0)}\left(x_{0}\right) \cdot \ldots \cdot \phi^{(n)}\left(x_{n}\right) \in \bar{\Sigma}^{*}
$$

definiert wie in Gleichung (5.2.3). Es gibt eine zu $\bar{\gamma}$ wählbare Konstante $l>0$ und eine Funktion $c(E)>0$, so daß für alle $x \in \mathcal{M}_{\mathrm{R}}^{n+1}$ gilt

$$
\left\|\phi^{(0)}\left(x_{0}\right) \cdot \ldots \cdot \phi^{(n)}\left(x_{n}\right)\right\|_{E} \leq c(E) \cdot \hat{d}(x)^{-2 n l} .
$$

Die Abbildung

$$
\Phi_{\bar{\gamma}}^{n+1} \rightarrow \bar{\Sigma}^{*}, \quad\left(\phi^{(0)}, \ldots, \phi^{(n)}\right) \mapsto \phi^{(0)}\left(x_{0}\right) \cdot \ldots \cdot \phi^{(n)}\left(x_{n}\right)
$$

ist (bei festem $x$ ) multilinear.

Die Multilinearität ist hierbei klar per Definition der Operatorprodukte als Grenzwerte. Sie führt dazu, daß wir die Definition der raumartigen Operatorprodukte auch auf Elemente des Tensorprodukts $\Phi_{\bar{\gamma}}^{\otimes n+1}$ ausdehnen können: Einer Linearkombination

$$
\Pi=\sum_{j} c_{j} \phi_{j}^{(0)} \otimes \ldots \otimes \phi_{j}^{(n)} \in \Phi_{\bar{\gamma}}^{\otimes n+1}
$$

ordnen wir das wohldefinierte Operatorprodukt

$$
\Pi(x)=\sum_{j} c_{j} \phi_{j}^{(0)}\left(x_{0}\right) \cdot \ldots \cdot \phi_{j}^{(n)}\left(x_{n}\right) \in \bar{\Sigma}^{*} \quad\left(x \in \mathcal{M}_{\mathrm{R}}^{n+1}\right)
$$

zu. Die Abschätzungen in Satz 5.1 gelten entsprechend. 
Wir bemerken noch, daß wir $\Pi(x)=\Pi^{\prime}(x)$ erhalten, falls $\Pi^{\prime}$ aus $\Pi$ durch Permutation der Tensorfaktoren hervorgeht - dies ergibt sich aus den Lokalitätseigenschaften der Operatorfolgen $A_{r}^{(j)}$. Das raumartige Produkt ist in diesem Sinne also kommutativ.

Die eigentliche Bedeutung der raumartigen Produkte liegt in ihrer Approximierbarkeit durch das Produkt lokaler Operatoren. Die gewonnenen Abschätzungen für die Konvergenzgeschwindigkeit werden wir auch in der späteren Argumentation noch verwenden; wir halten sie deshalb in einem eigenen Satz fest. Die funktionentheoretischen Details der Konstruktion spielen in der Anwendung allerdings keine Rolle mehr.

Satz 5.2 (Approximation raumartiger Operatorprodukte). Sei $\bar{\gamma}>0$ fest, und seien $\phi^{(0)}, \ldots, \phi^{(n)} \in \Phi_{\bar{\gamma}}$ lokale Punktfelder. Ist $l>0 z u \bar{\gamma}$ ausreichend groß, und sind $A_{r}^{(j)} \in \mathfrak{A}(r)$ Operatoren mit

$$
\left\|R^{l}\left(A_{r}^{(j)}-\phi^{(j)}\right) R^{l}\right\| \leq r \cdot \text { const. } \quad(j=0 \ldots n),
$$

dann gibt es eine Funktion $c^{\prime}(E)$, so daß für $x \in \mathcal{M}_{\mathrm{R}}^{n+1}$ und $r<\frac{1}{4} \hat{d}(x)$ die folgende Abschätzung gilt:

$$
\left\|\phi^{(0)}\left(x_{0}\right) \cdot \ldots \cdot \phi^{(n)}\left(x_{n}\right)-A_{r}^{(0)}\left(x_{0}\right) \cdot \ldots \cdot A_{r}^{(n)}\left(x_{n}\right)\right\|_{E} \leq c^{\prime}(E) \cdot r \cdot \hat{d}(x)^{-2 n l}
$$

Hierbei haben wir verwendet, daß die Konstruktion unabhängig von den zur Approximation verwendeten Operatorfolgen ist, solange sie die im Satz genannte Bedingung erfüllen.

\subsection{Produktentwicklung für raumartige Abstände}

Wir wollen nun die behauptete Operator-Produktentwicklung etablieren, und zwar für raumartig getrennte Argumente der Felder. Dazu werden wir das Produkt $\phi^{(0)}\left(x_{0}\right) \cdot \ldots$. $\phi^{(n)}\left(x_{n}\right)$ - wie im letzten Abschnitt definiert - in eine Reihe lokaler Punktfelder entwickeln, d.h. symbolisch

$$
\phi^{(0)}\left(x_{0}\right) \cdot \ldots \cdot \phi^{(n)}\left(x_{n}\right) \approx \sum_{j=1}^{J} c_{j}(x) \phi_{j}, \quad x \in \mathcal{M}_{\mathrm{R}}^{n+1}
$$

wobei sich herausstellt, daß die Koeffizienten $c_{j}(x)$ reguläre Funktionen (sogar holomorph fortsetzbare Funktionen) von $x$ sind.

Formaler suchen wir zu gegebenem $\beta>0$ eine Abbildung

$$
\varphi^{(\beta)}: \mathcal{M}_{\mathrm{R}}^{n+1} \rightarrow \Phi_{\bar{\gamma}} \quad(\bar{\gamma} \text { geeignet })
$$

so daß

$$
\left\|\phi^{(0)}\left(x_{0}\right) \cdot \ldots \cdot \phi^{(n)}\left(x_{n}\right)-\varphi^{(\beta)}\left(x_{0}, \ldots, x_{n}\right)\right\|_{E}\|x\|^{-\beta} \rightarrow 0
$$

wenn $x \rightarrow 0$ von raumartigen Abständen her (wir werden dies unten präzisieren). 
Skizze Zunächst sei hier die Vorgehensweise kurz skizziert, die zu solch einer Operatorproduktentwicklung führt. Nach den Ergebnissen des vorangegangenen Abschnitts lassen sich die raumartigen Produkte durch Folgen lokaler Operatoren approximieren:

$$
\phi^{(0)}\left(x_{0}\right) \cdot \ldots \cdot \phi^{(n)}\left(x_{n}\right) \approx \Xi\left(A_{r}^{(0)}\left(x_{0}\right) \cdot \ldots \cdot A_{r}^{(n)}\left(x_{n}\right)\right) \quad(r \rightarrow 0) .
$$

Der lokale Operator auf der rechten Seite läßt sich aufgrund der Phasenraumeigenschaften der Theorie nach lokalen Punktfeldern zerlegen: Ist $\bar{\gamma}>0$ und $p_{\bar{\gamma}}$ eine reguläre Projektion auf $\Phi_{\bar{\gamma}}$, dann gilt für kleine $r$ und $\left\|x_{j}\right\|$ :

$$
\Xi\left(A_{r}^{(0)}\left(x_{0}\right) \cdot \ldots \cdot A_{r}^{(n)}\left(x_{n}\right)\right) \approx p_{\bar{\gamma}} \circ \Xi\left(A_{r}^{(0)}\left(x_{0}\right) \cdot \ldots \cdot A_{r}^{(n)}\left(x_{n}\right)\right) .
$$

Die Projektion $p_{\bar{\gamma}}$ ist „energiebeschränkt“ (sie läßt sich als eine Summe mit energiebeschränkten Funktionalen schreiben), weswegen die Folge der lokalen Operatoren unter $p_{\bar{\gamma}}$ wieder konvergiert:

$$
p_{\bar{\gamma}} \circ \Xi\left(A_{r}^{(0)}\left(x_{0}\right) \cdot \ldots \cdot A_{r}^{(n)}\left(x_{n}\right)\right) \approx p_{\bar{\gamma}}\left(\phi^{(0)}\left(x_{0}\right) \cdot \ldots \cdot \phi^{(n)}\left(x_{n}\right)\right) .
$$

Damit erhalten wir insgesamt:

$$
\phi^{(0)}\left(x_{0}\right) \cdot \ldots \cdot \phi^{(n)}\left(x_{n}\right) \approx p_{\bar{\gamma}}\left(\phi^{(0)}\left(x_{0}\right) \cdot \ldots \cdot \phi^{(n)}\left(x_{n}\right)\right) .
$$

Das ist die gewünschte Produktentwicklung, wenn wir

$$
\varphi^{(\beta)}\left(x_{0}, \ldots, x_{n}\right):=p_{\bar{\gamma}}\left(\phi^{(0)}\left(x_{0}\right) \cdot \ldots \cdot \phi^{(n)}\left(x_{n}\right)\right)
$$

setzen. Die approximierende Abbildung $\varphi^{(\beta)}(x)$ ist also sehr direkt bekannt, deshalb sind viele ihrer Eigenschaften auch leicht zugänglich.

Präzisierung Es geht nun darum, die eben skizzierte Approximation genau zu quantifizieren und die Details des Limesprozesses zu klären.

Es seien dazu $n+1$ Punktfelder $\phi^{(0)}, \ldots, \phi^{(n)} \in \Phi_{\bar{\gamma}}$ gegeben $(\bar{\gamma}$ fest). Wir verschaffen uns nach Satz 3.17 zunächst approximierende Folgen von Punktfelder $A_{r}^{(j)} \in \mathfrak{A}(r)$ mit

$$
\left\|A_{r}^{(j)}\right\| \leq r^{-\kappa} \cdot \text { const. }, \quad\left\|R^{l}\left(\phi^{(j)}-A_{r}^{(j)}\right) R^{l}\right\| \leq r \cdot \text { const. }
$$

die wir im folgenden festhalten. $(l>0, \kappa>0$ sind Konstanten, die durch Wahl einer Basis gleichmäßig für $\phi^{(j)} \in \Phi_{\bar{\gamma}}$ bei festem $\bar{\gamma}$ gewählt werden können.) Zur Vereinfachung schreiben wir

$$
\begin{aligned}
& \phi^{(0 \ldots n)}(x):=\phi^{(0)}\left(x_{0}\right) \cdot \ldots \cdot \phi^{(n)}\left(x_{n}\right), \\
& A_{r}^{(0 \ldots n)}(x):=A_{r}^{(0)}\left(x_{0}\right) \cdot \ldots \cdot A_{r}^{(n)}\left(x_{n}\right) \quad\left(x \in \mathcal{M}_{\mathrm{R}}^{n+1}\right) \text {. }
\end{aligned}
$$

Wir zeigen zunächst, daß $\phi^{(0 \ldots n)}(x) \approx \Xi\left(A_{r}^{(0 \ldots n)}(x)\right)$. Dies ergibt sich mit Satz 5.2: Wir erhalten daraus, daß für festes $E>0$ gilt

$$
\left\|\phi^{(0 \ldots n)}(x)-A_{r}^{(0 \ldots n)}(x)\right\|_{E} \leq r \cdot \hat{d}(x)^{-2 n l} \cdot \text { const. } \quad \text { für } r<\frac{1}{4} \hat{d}(x) .
$$


Die auftretende Konstante wird i.allg. von $E$ abhängen, was wir aber nicht weiter notieren.

Der nächste Schritt lautet $\Xi\left(A_{r}^{(0 \ldots n)}(x)\right) \approx p_{\bar{\gamma}^{\prime}} \circ \Xi\left(A_{r}^{(0 \ldots n)}(x)\right)$ : Es sei $\bar{\gamma}^{\prime}>0$ (wir werden diese Wahl noch genauer festlegen). Ist dann $p_{\bar{\gamma}^{\prime}}: \bar{\Sigma}^{*} \rightarrow \Phi_{\bar{\gamma}^{\prime}}$ eine reguläre Projektion, so gilt nach Satz 4.2 bei festem $E$ und $\epsilon>0$ :

$$
\left\|\Xi\left(A_{r}^{(0 \ldots n)}(x)\right)-p_{\bar{\gamma}^{\prime}} \circ \Xi\left(A_{r}^{(0 \ldots n)}(x)\right)\right\|_{E} \leq(r+2\|x\|)^{\bar{\gamma}^{\prime}-\epsilon}\left\|A_{r}^{(0 \ldots n)}(x)\right\| \cdot \text { const. },
$$

wobei man bemerkt, $\operatorname{da} \beta A_{r}^{(0 \ldots n)}(x) \in \mathfrak{A}(r+2\|x\|)$. Mit $\epsilon:=1$ haben wir also

$$
\left\|\Xi\left(A_{r}^{(0 \ldots n)}(x)\right)-p_{\bar{\gamma}^{\prime}} \circ \Xi\left(A_{r}^{(0 \ldots n)}(x)\right)\right\|_{E} \leq(r+2\|x\|)^{\bar{\gamma}^{\prime}-1} r^{-(n+1) \kappa} \cdot \text { const. }^{\prime}
$$

Nun bleibt noch die dritte Approximation zu behandeln: Wir schreiben $p_{\bar{\gamma}^{\prime}}$ durch Wahl einer Basis aus als

$$
p_{\bar{\gamma}^{\prime}}=\sum_{j=1}^{J} \sigma_{j}(\cdot) \phi_{j} \quad \text { mit } \phi_{j} \in \Phi_{\bar{\gamma}^{\prime}} \text { und } \sigma_{j} \in \Sigma\left(E^{\prime}\right), E^{\prime}>0 \text { geeignet. }
$$

Ein analoges Argument wie in (5.3.11) führt uns dann auf die Abschätzung

$$
\left\|p_{\bar{\gamma}^{\prime}} \circ \Xi\left(A_{r}^{(0 \ldots n)}(x)\right)-p_{\bar{\gamma}^{\prime}}\left(\phi^{(0 \ldots n)}(x)\right)\right\|_{E} \leq r \cdot \hat{d}(x)^{-2 n l} \cdot \text { const. für } r<\frac{1}{4} \hat{d}(x) .
$$

Die drei Abschätzungen (5.3.11), (5.3.13) und (5.3.15) haben wir jetzt im angesprochenen Grenzfall zu kontrollieren. Dieser wird präzise so formuliert: Für eine Folge $\left(x^{(k)}\right) \subset$ $\mathcal{M}_{\mathrm{R}}^{n+1}$ schreiben wir

$$
x^{(k)} \underset{\mathrm{R}}{\longrightarrow} 0 \quad\left(, x^{(k)} \text { strebt raumartig gegen } 0^{“}\right)
$$

wenn

$$
x^{(k)} \rightarrow 0 \quad \text { und } \quad \hat{d}\left(x^{(k)}\right) \geq\left\|x^{(k)}\right\| \cdot \text { const. }
$$

d.h. wenn die Folge sich nicht zu schnell dem Rand von $\mathcal{M}_{\mathrm{R}}^{n+1}$ nähert.5 Der betrachtete Grenzübergang soll diese Form haben.

Es sei nun $\beta>0$ gegeben. Im betrachteten Limesfall gilt dann nach (5.3.11):

$$
\|x\|^{-\beta}\left\|\phi^{(0 \ldots n)}(x)-A_{r}^{(0 \ldots n)}(x)\right\|_{E} \leq r \cdot\|x\|^{-2 n l-\beta} \cdot \text { const. }
$$

Dies verschwindet im Limes $x \underset{\mathrm{R}}{\longrightarrow} 0$, wenn wir $r(x):=\|x\|^{2 n l+\beta+1}$ setzen. Die Bedingung $r<\frac{1}{4} \hat{d}(x)$ ist dabei für kleine $\|x\|$ sicherlich erfüllt. Mit dieser Festsetzung wird weiterhin

$$
\begin{aligned}
&\|x\|^{-\beta} \| \Xi\left(A_{r}^{(0 \ldots n)}(x)\right)-p_{\bar{\gamma}^{\prime}} \circ \Xi\left(A_{r}^{(0 \ldots n)}(x)\right) \|_{E} \\
& \underset{(5.3 .13)}{\leq}\left(\|x\|^{2 n l+\beta+1}+2\|x\|\right)^{\bar{\gamma}^{\prime}-1}\|x\|^{-(2 n l+\beta+1) \cdot(n+1) \kappa-\beta} \cdot \text { const. },
\end{aligned}
$$

was sicher verschwindet, wenn $\bar{\gamma}^{\prime}$ genügend groß gewählt war. Der Ausdruck (5.3.15) verschwindet ebenfalls schneller als $\|x\|^{\beta}$, weshalb wir insgesamt erhalten:

$$
\|x\|^{-\beta}\left\|\phi^{(0 \ldots n)}(x)-p_{\bar{\gamma}^{\prime}}\left(\phi^{(0 \ldots n)}(x)\right)\right\|_{E} \underset{\mathrm{R}}{\longrightarrow} 0 .
$$

Wir formulieren dies als Satz:

\footnotetext{
${ }^{5}$ Insbesondere erfüllt eine Folge $x^{(k)}=\lambda_{k} x$ mit festem $x \in \mathcal{M}_{\mathrm{R}}^{n+1}$ und $\lambda_{k} \rightarrow 0$ die genannte Bedingung.
} 
Satz 5.3. Es seien $\bar{\gamma}>0, \phi^{(0)}, \ldots, \phi^{(n)} \in \Phi_{\bar{\gamma}}$ und $\beta>0$ gegeben. Für zu $\bar{\gamma}$, $n$ und $\beta$ genügend großes $\bar{\gamma}^{\prime}>0$ und jede reguläre Projektion $p_{\bar{\gamma}^{\prime}}$ auf $\Phi_{\bar{\gamma}^{\prime}}$ gilt dann

$$
\|x\|^{-\beta}\left\|\phi^{(0 \ldots n)}(x)-p_{\bar{\gamma}^{\prime}}\left(\phi^{(0 \ldots n)}(x)\right)\right\|_{E \underset{\mathrm{R}}{\longrightarrow}} 0 \quad \forall E>0 .
$$

Das ist die gewünschte Operatorproduktentwicklung. Sie läßt sich in der gewohnten Form $\phi^{(0 \ldots n)}(x) \approx \sum c_{j}(x) \phi_{j}$ schreiben, indem man eine Basisdarstellung der Projektion $p_{\bar{\gamma}^{\prime}}$ wählt. Die „Koeffizientenfunktionen“ $c_{j}(x)$ erhält man allgemein durch Anwenden eines Funktionals $v^{*} \in \Phi_{\bar{\gamma}^{\prime}}^{*}$ auf den Approximationsterm:

$$
c_{v^{*}}:=v^{*} \circ p_{\bar{\gamma}^{\prime}}\left(\phi^{(0 \ldots n)}(x)\right) .
$$

Die $p_{\bar{\gamma}^{\prime}}$ und damit die Koeffizientenfunktionen sind in unserem Rahmen nicht eindeutig bestimmt; man könnte sowohl $\bar{\gamma}^{\prime}$ wie die Projektion $p_{\bar{\gamma}^{\prime}}$ noch variieren. Die Approximationseigenschaft in (5.3.20) legt den Term $p_{\bar{\gamma}^{\prime}}\left(\phi^{(0 \ldots n)}(x)\right)$ nur bis auf Anteile fest, die im Limes schneller als $\|x\|^{\beta}$ verschwinden. Erhöht man die „Approximationsgenauigkeit“ $\beta$, so muß auch der Approximationsterm angepaßt werden; ihn für alle $\beta$ fest zu wählen, scheint in unserem Rahmen im allgemeinen nicht möglich. Verzichtet man allerdings wie in [W772] darauf, die lineare Unabhängigkeit der Felder $\phi_{j}$ in einer Reihenentwicklung $\phi^{(0 \ldots n)}(x) \approx \sum c_{j}(x) \phi_{j}$ zu fordern, so kann man eine solche Reihe, die „für alle $\beta^{\text {“ appro- }}$ ximiert, trivialerweise durch Basisentwicklung von

$$
p_{\bar{\gamma}_{1}}\left(\phi^{(0 \ldots n)}(x)\right)+\left(p_{\bar{\gamma}_{2}}-p_{\bar{\gamma}_{1}}\right)\left(\phi^{(0 \ldots n)}(x)\right)+\left(p_{\bar{\gamma}_{3}}-p_{\bar{\gamma}_{2}}\right)\left(\phi^{(0 \ldots n)}(x)\right)+\ldots
$$

erhalten, wobei $\bar{\gamma}_{j} \rightarrow \infty$.

Die auftretende Mehrdeutigkeit der Approximationsterme ist, wie erwähnt, auf im Limes schnell verschwindende Anteile beschränkt. Insbesondere ist die Singularitätenstruktur der Operatorproduktentwicklung unabhängig von den verbleibenden Ambiguitäten und kann daher als intrinsische Eigenschaft der Theorie verstanden werden.

Über die Koeffizientenfunktionen $c_{v^{*}}(x)$ haben wir aus Abschnitt 5.2 detaillierte Aussagen zur Verfügung. Zunächst sind die $c_{v^{*}}(x)$ holomorph fortsetzbar auf eine komplexe Umgebung von $\mathcal{M}_{\mathrm{R}}^{n+1}$. Ferner wachsen sie für $x \underset{\mathrm{R}}{\longrightarrow} 0$ höchstens wie eine inverse Potenz von $\|x\|$ an, wie man aus Satz 5.1 entnimmt. Tatsächlich folgt aus der Approximationseigenschaft sogar, daß in einer Summendarstellung $\phi^{(0 \ldots n)}(x) \approx \sum c_{j}(x) \phi_{j}$ nur eine feste (von $\beta$ unabhängige) Zahl von Funktionen tatsächlich divergieren können, während die übrigen für $x \underset{\mathrm{R}}{\longrightarrow} 0$ gegen 0 konvergieren. Auf diesen Aspekt werden wir in Kapitel 6 näher eingehen, um ein Normalprodukt der Felder am gleichen Raum-Zeit-Punkt zu definieren.

Wir bemerken noch, daß auch die Operatorproduktentwicklung (wie die Produkte selbst) in natürlicher Weise auf Linearkombinationen $\Pi \in \Phi_{\bar{\gamma}}^{\otimes n+1}$ ausgedehnt werden kann; Satz 5.3 überträgt sich wörtlich.

Ein Beispiel für die Berechnung der raumartigen Produkte und der Produktentwicklung in der freien Feldtheorie behandeln wir in Abschnitt 7.5 .

\subsection{Symmetrieeigenschaften der Approximationsterme}

In den Anwendungen der Operatorproduktentwicklung spielen ihre Symmetrieeigenschaften eine wesentliche Rolle. So wird die Produktentwicklung in der störungstheoretischen 
Behandlung der starken Wechselwirkung verwendet, um deren Hochenergielimes zu analysieren, in dem (approximativ) eine Dilatationsinvarianz auftritt [Wil69].

Es stellt sich also die Frage, inwieweit sich Symmetrien der zugrundeliegenden Theorie in der Produktentwicklung wiederspiegeln, das heißt in unserem Kontext, wie sich bei gegebenem $\Pi \in \Phi_{\bar{\gamma}}^{\otimes n+1}$ die Ausdrücke $\Pi(x)$ und $p_{\bar{\gamma}^{\prime}} \Pi(x)$ unter lokalen Transformationen $\alpha$ verhalten.

Dabei haben wir auf dem Tensorprodukt $\Phi_{\bar{\gamma}}^{\otimes n+1}$ eine natürliche Darstellung von $\alpha$ durch

$$
\alpha\left(\phi^{(0)} \otimes \ldots \otimes \phi^{(n)}\right)=\alpha \phi^{(0)} \otimes \ldots \otimes \alpha \phi^{(n)}
$$

gegeben; auch auf den Linearformen $\Pi(x) \in \bar{\Sigma}^{*}$ wirkt $\alpha$ in bekannter Weise. Um aber zeigen zu können, daß die raumartigen Produkte $\Pi(x)$ durch $\alpha$ wieder in solche überführt werden, muß man die Kompatibilität der beiden Darstellungen untersuchen, d.h. man muß eine Relation der Art

$$
\alpha(\Pi(x))=(\alpha \Pi)(x)
$$

etablieren. Hierzu werden außer den bisher betrachteten Axiomen für lokale Transformationen noch weitere Zusatzannahmen benötigt: Einerseits sollte $\alpha$ die Produktstruktur in $\mathfrak{B}(\mathcal{H})$ respektieren, andererseits muß man eine gewisse Verträglichkeit mit der Darstellung der Translationen sicherstellen. Konkret fordern wir folgendes:

Definition 5.4. Eine lokale Transformation $\alpha$ heiße verträglich mit raumartigen Produkten, wenn sie

$$
\alpha(A B)=\alpha(A) \alpha(B) \quad \forall A, B \in \mathfrak{B}(\mathcal{H})
$$

erfüllt, und wenn es eine Operation $x \mapsto \alpha . x$ von $\alpha$ auf $\mathcal{M}$ gibt, so daß

$$
\begin{aligned}
\alpha \circ \operatorname{ad} U(x) & =\operatorname{ad} U(\alpha \cdot x) \circ \alpha \quad(x \in \mathcal{M}), \\
\alpha \cdot \mathcal{M}_{\mathrm{R}}^{n+1} & \subset \mathcal{M}_{\mathrm{R}}^{n+1}, \\
\text { und } \quad \alpha . x \underset{\mathrm{R}}{\longrightarrow} 0 & \Leftrightarrow \quad x \underset{\mathrm{R}}{\longrightarrow} 0 \quad\left(x \in \mathcal{M}_{\mathrm{R}}^{n+1}\right) .
\end{aligned}
$$

(Hierbei wirkt $\alpha$ auf jede der $n+1$ „Minkowski-Komponenten " von $x \in \mathcal{M}_{\mathrm{R}}^{n+1}$.)

Beispiele für solche Transformationen sind:

- Die unitär implementierten Darsteller der Lorentzgruppe (mit $\alpha(\Lambda) \cdot x=\Lambda x)$;

- eine unitäre Darstellung der Dilatationen $(\operatorname{mit} \alpha(\lambda) \cdot x=\lambda x)$;

- innere Symmetrien der Theorie, wenn sie durch Automorphismen von $\mathfrak{B}(\mathcal{H})$ wirken und mit den Translationen kommutieren $(\alpha \cdot x=x)$.

Wir zeigen nun für solche Transformationen die Verträglichkeitseigenschaft (5.4.2) in leichter Abwandlung.

Lemma 5.5. Es sei $\alpha$ eine lokale Transformation, die verträglich mit raumartigen Produkten ist. Dann gilt für $\Pi \in \Phi_{\bar{\gamma}}^{\otimes n+1}$ :

$$
\alpha(\Pi(x))=(\alpha \Pi)(\alpha \cdot x) .
$$


Beweis. Es reicht aus Linearitätsgründen aus, $\Pi=\phi^{(0)} \otimes \ldots \otimes \phi^{(n)}$ zu betrachten. Wir wählen approximierende Folgen $A_{r}^{(j)} \mathrm{zu} \phi^{(j)}$ wie in Satz 3.17. Für $\sigma \in \Sigma(E)$ gilt dann nach Satz 5.2

$$
\sigma\left(\alpha\left(\phi^{(0 \ldots n)}(x)\right)\right)=\lim _{r \rightarrow 0} \sigma\left(\alpha\left(A_{r}^{(0 \ldots n)}(x)\right)\right),
$$

da $\sigma \circ \alpha \in \Sigma(\lambda E)$ mit geeignetem $\lambda$. Die Verträglichkeit von $\alpha$ mit Produktstruktur und Translationen impliziert

$$
\alpha\left(A_{r}^{(0 \ldots n)}(x)\right)=\alpha A_{r}^{(0)}\left(\alpha . x_{0}\right) \cdot \ldots \cdot \alpha A_{r}^{(n)}\left(\alpha . x_{n}\right) .
$$

Können wir nun noch zeigen, daß

$$
\left\|R^{l}\left(\alpha A_{r}^{(j)}-\alpha \phi^{(j)}\right) R^{l}\right\| \leq r \cdot \text { const. }
$$

für genügend großes $l$, dann folgt nach Satz 5.2:

$$
\sigma\left(\alpha A_{r}^{(0 \ldots n)}(x)\right) \underset{r \rightarrow 0}{\longrightarrow} \sigma\left(\alpha \phi^{(0)}\left(\alpha . x_{0}\right) \cdot \ldots \cdot \alpha \phi^{(n)}\left(\alpha . x_{n}\right)\right)=\sigma((\alpha \Pi)(\alpha . x)) .
$$

Daraus ergibt sich dann die Behauptung des Lemmas. Zum Beweis von (5.4.5) geht man so vor: Wir wissen, daß

$$
\left\|R^{l}\left(A_{r}^{(j)}-\phi^{(j)}\right) R^{l}\right\| \leq r \cdot c
$$

mit einer Konstanten $c>0$; also gilt

$$
\left\|A_{r}^{(j)}-\phi^{(j)}\right\|_{E} \leq(1+E)^{2 l} \cdot r \cdot c^{\prime} \quad \forall E>0 \quad\left(c^{\prime}>0 \text { konstant }\right) .
$$

Ist $\sigma \in \Sigma(E)$, dann hat man

$$
\sigma \circ \alpha \in \Sigma(\lambda E) \quad \text { und } \quad\|\sigma\|=\|\sigma \circ \alpha\|
$$

da $\alpha$ eine lokale Transformation ist; das impliziert

$$
\|\alpha \cdot\|_{E} \leq\|\cdot\|_{\lambda E}
$$

Man erhält so für $E>0$ :

$$
\left\|P(E) \alpha\left(A_{r}^{(j)}-\phi^{(j)}\right) P(E)\right\| \leq\left\|A_{r}^{(j)}-\phi^{(j)}\right\|_{\lambda E} \leq \lambda^{2 l} r c^{\prime}(1+E)^{2 l} .
$$

Nach Lemma 3.27 erhalten wir damit eine Konstante $c^{\prime \prime}$, so daß

$$
\left\|R^{2 l+1} \alpha\left(A_{r}^{(j)}-\phi^{(j)}\right) R^{2 l+1}\right\| \leq r \cdot c^{\prime \prime}
$$

wie gewünscht.

Wir wenden diese Ergebnisse jetzt auf die raumartige Produktentwicklung an. Wieder sei $\bar{\gamma}>0$ fest und $\alpha$ eine Transformation nach Definition 5.4. Dann ist mit $p_{\bar{\gamma}^{\prime}}$ auch $\alpha^{-1} p_{\bar{\gamma}^{\prime}} \alpha$ eine reguläre Projektion auf $\Phi_{\bar{\gamma}^{\prime}}($ vgl. Satz 4.6$)$. Für $\Pi \in \Phi_{\bar{\gamma}}^{\otimes n+1}$ hat man damit

$$
\|x\|^{-\beta}\left\|\Pi(x)-p_{\bar{\gamma}^{\prime}} \Pi(x)\right\|_{E} \underset{\mathrm{R}}{\longrightarrow} 0 \quad \forall E>0
$$

wie auch $\quad\|x\|^{-\beta}\left\|\Pi(x)-\alpha^{-1} p_{\bar{\gamma}^{\prime}} \alpha \Pi(x)\right\|_{E} \underset{\mathrm{R}}{\longrightarrow} 0 \quad \forall E>0$. 
Per Dreiecksungleichung und nach linksseitiger Anwendung von $\alpha$ ergibt sich daraus

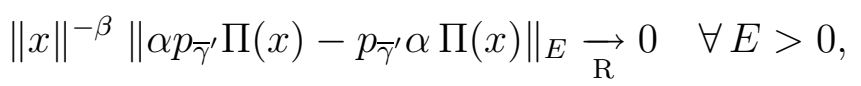

oder in etwas suggestiver Schreibweise:

$$
\alpha p_{\bar{\gamma}^{\prime}} \Pi(x) \approx p_{\bar{\gamma}^{\prime}}((\alpha \Pi)(\alpha \cdot x)) .
$$

Die Approximationsterme der Operatorproduktentwicklung spiegeln also (bis auf schnell verschwindende Anteile, die sich in unserem Zugang nicht ausschließen lassen) die Symmetrien der Theorie wieder. Man beachte dabei, daß $\alpha$ sowohl auf der linken Seite der Gleichung wie auch in den einzelnen Tensorfaktoren von $\Pi$ durch eine endlichdimensionale Matrixdarstellung wirkt. Schreibt man $p_{\bar{\gamma}^{\prime}}$ bezüglich einer Basis aus, dann kann die Wirkung von $\alpha$ als Transformation der Koeffizientenfunktionen aufgefaßt werden.

Diese Kovarianzeigenschaften sind in den Anwendungen der Operatorproduktentwicklung (vgl. [Wei96, ch. 20]) ein zentraler Aspekt, vor allem im Hinblick auf spontan gebrochene Symmetrien: Solche Transformationen lassen den Vakuumzustand nicht invariant; da die Phasenraumapproximation und die Produktentwicklung aber auf keinen speziellen Zustand Bezug nehmen, sind die Symmetrien in den Termen der Entwicklung trotzdem sichtbar, sofern sie unsere Voraussetzungen erfüllen.

Besonders interessant ist dies im Zusammenhang mit spontan gebrochenen inneren (Eich-)Symmetrien. Zu deren Behandlung betrachtet man statt der Observablenalgebren $\mathfrak{A}(\mathcal{O})$ die Feldalgebren $\mathfrak{F}(\mathcal{O})$; unser Formalismus läßt sich auch auf diese anwenden, nach eventueller Modifikation beim Auftreten von Fermi-Feldern (vgl. Abschnitt 8.1). Man erwartet dann, daß auch die gebrochenen Symmetrien als Automorphismen auf die beschränkten Operatoren wirken, zumindest auf den lokalen Feldalgebren $\mathfrak{F}(r)$ [BDLIR.92], was für unsere Zwecke ausreichen würde.

\subsection{Produktentwicklung im Sinne von Distributionen}

Wir wollen nun die oben entwickelte Methode modifizieren, um eine Operatorproduktentwicklung für beliebige (nicht notwendig raumartige) Abstände der Argumente zu etablieren. Hier ist das Produkt der Felder allerdings nur noch im Sinne von Distributionen definiert, und auch die „Koeffizienten“ der Operatorproduktentwicklung werden im allgemeinen Distributionen sein.

Es seien wieder $\bar{\gamma}>0$ fest und $\phi^{(0)}, \ldots, \phi^{(n)} \in \Phi_{\bar{\gamma}}$. Das Produkt dieser Felder nach „Verschmierung“ mit Testfunktionen liefert a priori eine wohldefinierte Linearform auf $\bar{\Sigma}^{*}$ :

$$
\phi^{(0 \ldots n)}(g):=\phi^{(0)}\left(g_{0}\right) \cdot \ldots \cdot \phi^{(n)}\left(g_{n}\right), \quad g_{0}, \ldots, g_{n} \in \mathcal{S}(\mathcal{M}) .
$$

Diese Linearform soll im Limes immer besser lokalisierter Testfunktionen $g_{j}$ analysiert werden. Präziser verlangen wir, daß die $g_{j}$ kompakten Träger besitzen; setzen wir

$$
d(g):=\max _{j} \sup \left\{\|x\| \mid x \in \operatorname{supp} g_{j}\right\},
$$

dann soll nicht nur $d(g)$ im Limes verschwinden, sondern es gelte dabei auch

$$
\left\|\partial_{\mu} g_{j}\right\|_{1} \leq d(g)^{-|\mu|} \cdot c_{\mu} \quad \forall \mu \in \mathfrak{M}^{s+1}, j=0 \ldots n .
$$


(Hier ist $\|\cdot\|_{1}$ die $L_{1}$-Norm, $\mu$ ein Multiindex; $c_{\mu}$ sind Konstanten.) Wir sprechen in diesem Fall auch vom Limes scharf lokalisierter Testfunktionen. 5 Beispielsweise sind die genannten Bedingungen erfüllt, wenn wir

$$
g_{j}(x)=\frac{1}{\lambda} g_{j, 0}\left(\frac{x}{\lambda}\right), \quad \lambda>0
$$

mit einer festen Testfunktion $g_{j, 0}$ mit kompaktem Träger im Limes $\lambda \rightarrow 0$ betrachten.

Um das Produkt $\phi^{(0 \ldots n)}(g)$ mit Hilfe des Phasenraumkriteriums analysieren zu können, approximieren wir es wieder durch lokale Operatoren: Wir wählen nach Satz 3.17 approximierende Folgen $A_{r}^{(j)} \in \mathfrak{A}(r)$, ein $l>0$ und ein $\kappa>0$, so daß

$$
\left\|A_{r}^{(j)}\right\| \leq r^{-\kappa} \cdot \text { const. }, \quad\left\|R^{l}\left(\phi^{(j)}-A_{r}^{(j)}\right) R^{l}\right\| \leq r \cdot \text { const. }
$$

Außerdem sei $\left\|R^{l} \phi^{(j)} R^{l}\right\|<\infty$ für alle $j$. Die Konstanten $l$ und $\kappa$ hängen nur von $\bar{\gamma}$ ab. Es ist dann intuitiv klar, daß

$$
A_{r}^{(0)}\left(g_{0}\right) \cdot \ldots \cdot A_{r}^{(n)}\left(g_{n}\right)=: A_{r}^{(0 \ldots n)}(g) \underset{r \rightarrow 0}{\longrightarrow} \phi^{(0 \ldots n)}(g)
$$

Wir wollen dies genauer quantifizieren. Dazu schreiben wir das Produkt der WightmanFelder in anderer Form: Bei festem $E$ erhalten wir durch mehrfaches Ausnutzen der Kommutatorrelation aus Lemma 3.28 eine Darstellung

$$
\begin{aligned}
P(E) \phi^{(0)}\left(g_{0}\right) & \ldots \cdot \phi^{(n)}\left(g_{n}\right) P(E) \\
= & \sum_{\text {endl. }} c_{k l m} P(E) R^{-k}\left(R^{l_{0}} \phi^{(0)}\left(\partial_{t}^{m_{0}} g_{0}\right) R^{l_{1}} \phi^{(1)}\left(\partial_{t}^{m_{1}} g_{1}\right) R^{l_{2}} \cdot \ldots\right. \\
& \left.\cdot R^{l_{n}} \phi^{(n)}\left(\partial_{t}^{m_{n}} g_{n}\right) R^{l_{n+1}}\right) R^{-k^{\prime}} P(E) .
\end{aligned}
$$

Hierbei läuft die (endliche) Summe über gewisse Parameter $l_{j}, m_{j}, k, k^{\prime}$; die $c_{k l m}$ sind Phasenfaktoren. Wir interessieren uns nicht für die Details dieser Entwicklung; wichtig ist nur, daß man $l_{j} \geq 2 l$ für alle $j$ erreichen kann, und daß dabei $m_{j} \leq 2 n l$ gilt.

Für $A_{r}^{(0 \ldots n)}(g)$ statt $\phi^{(0 \ldots n)}(g)$ erhält man wörtlich dieselbe Entwicklung, mit gleichen Parametern. Wir betrachten daher die Differenz zweier Summanden dieser Entwicklungen: Es ist

$$
\begin{aligned}
& \| R^{l_{0}} \phi^{(0)}\left(\partial_{t}^{m_{0}} g_{0}\right) R^{l_{1}} \ldots \cdot R^{l_{n}} \phi^{(n)}\left(\partial_{t}^{m_{n}} g_{n}\right) R^{l_{n+1}} \\
& -R^{l_{0}} A_{r}^{(0)}\left(\partial_{t}^{m_{0}} g_{0}\right) R^{l_{1}} \cdot \ldots \cdot R^{l_{n}} A_{r}^{(n)}\left(\partial_{t}^{m_{n}} g_{n}\right) R^{l_{n+1}} \| \\
\leq & \left\|R^{l}\left(\phi^{(0)}-A_{r}^{(0)}\right)\left(\partial_{t}^{m_{0}} g_{0}\right) R^{l}\right\| \cdot\left\|R^{l} \phi^{(1)}\left(\partial_{t}^{m_{1}} g_{1}\right) R^{l}\right\| \cdot \ldots \cdot\left\|R^{l} \phi^{(n)}\left(\partial_{t}^{m_{n}} g_{n}\right) R^{l}\right\| \\
+ & \left\|R^{l} A_{r}^{(0)}\left(\partial_{t}^{m_{0}} g_{0}\right) R^{l}\right\| \cdot\left\|R^{l}\left(\phi^{(1)}-A_{r}^{(1)}\right)\left(\partial_{t}^{m_{1}} g_{1}\right) R^{l}\right\| \cdot \ldots \cdot\left\|R^{l} \phi^{(n)}\left(\partial_{t}^{m_{n}} g_{n}\right) R^{l}\right\| \\
+ & \ldots \\
+ & \left\|R^{l} A_{r}^{(0)}\left(\partial_{t}^{m_{0}} g_{0}\right) R^{l}\right\| \cdot\left\|R^{l} A_{r}^{(1)}\left(\partial_{t}^{m_{1}} g_{1}\right) R^{l}\right\| \cdot \ldots \cdot\left\|R^{l}\left(\phi^{(n)}-A_{r}^{(n)}\right)\left(\partial_{t}^{m_{n}} g_{n}\right) R^{l}\right\| .
\end{aligned}
$$

${ }^{6}$ Die Bedingungen für den Limes könnten, wie unten deutlich wird, auch noch weiter abgeschwächt werden. 
Hier können wir folgendermaßen abschätzen: Man hat

$$
\begin{aligned}
\left\|R^{l} \phi^{(j)}\left(\partial_{t}^{m_{j}} g_{j}\right) R^{l}\right\| & \leq\left\|\partial_{t}^{m_{j}} g_{j}\right\|_{1} \cdot \text { const. } \\
\left\|R^{l} A_{r}^{(j)}\left(\partial_{t}^{m_{j}} g_{j}\right) R^{l}\right\| & \leq\left\|\partial_{t}^{m_{j}} g_{j}\right\|_{1} \cdot \text { const. für kleine } r
\end{aligned}
$$

und aufgrund der Approximationseigenschaft (5.5.5) erhält man

$$
\left\|R^{l}\left(\phi^{(j)}-A_{r}^{(j)}\right)\left(\partial_{t}^{m_{j}} g_{j}\right) R^{l}\right\| \leq\left\|\partial_{t}^{m_{j}} g_{j}\right\|_{1} \cdot r \cdot \text { const. }
$$

Berücksichtigen wir, daß im Limes die Bedingung (5.5.3) für die Testfunktionen und ihre Ableitungen gelten soll, dann erhalten wir für (5.5.8) die Abschätzung

$$
\|\ldots\| \leq r \cdot d(g)^{-2 n(n+1) l} \cdot \text { const. }
$$

und nach Aufsummation in (5.5.7) ergibt sich daraus

$$
\left\|\phi^{(0 \ldots n)}(g)-A_{r}^{(0 . . n)}(g)\right\|_{E} \leq r \cdot d(g)^{-2 n(n+1) l} \cdot \text { const. }(E) \quad \forall E>0 .
$$

Damit können wir die Approximation der $\phi^{(0 \ldots n)}(g)$ durch lokale Operatoren kontrollieren. Diese Operatoren werden jetzt in die Phasenraumentwicklung eingesetzt: Da $A_{r}^{(0 \ldots n)}(g)$ in der Algebra $\mathfrak{A}(2 d(g)+r)$ liegt, erhält man nach Wahl eines $\bar{\gamma}^{\prime}>0$ :

$$
\begin{array}{r}
\left\|A_{r}^{(0 \ldots n)}(g)-p_{\bar{\gamma}^{\prime}} A_{r}^{(0 \ldots n)}(g)\right\|_{E} \leq\left\|\Xi-p_{\bar{\gamma}^{\prime}} \Xi\right\|_{E, 2 d(g)+r} \cdot r^{-(n+1) \kappa} \cdot \prod_{j}\left\|g_{j}\right\|_{1} \\
\underset{(\operatorname{Satz}[.2)}{\leq}(2 d(g)+r)^{\bar{\gamma}^{\prime}-1} r^{-(n+1) \kappa} \cdot \operatorname{const.}(E) .
\end{array}
$$

Hierbei ist $p_{\bar{\gamma}^{\prime}}$ eine beliebig gewählte reguläre Projektion auf $\Phi_{\bar{\gamma}^{\prime}}$. Setzen wir (5.5.13) und (5.5.14) zusammen, dann ergibt sich also:

$$
\begin{aligned}
\| \phi^{(0 \ldots n)}(g) & -p_{\bar{\gamma}^{\prime}} \phi^{(0 \ldots n)}(g)\left\|_{E} \leq\right\| \phi^{(0 \ldots n)}(g)-A_{r}^{(0 \ldots n)}(g) \|_{E} \\
& +\left\|A_{r}^{(0 \ldots n)}(g)-p_{\bar{\gamma}^{\prime}} A_{r}^{(0 \ldots n)}(g)\right\|_{E}+\left\|p_{\bar{\gamma}^{\prime}}\right\| \cdot\left\|A_{r}^{(0 \ldots n)}(g)-\phi^{(0 \ldots n)}(g)\right\|_{E^{\prime}}
\end{aligned}
$$

(Die Konstante $E^{\prime}$ wird durch $p_{\bar{\gamma}^{\prime}}$ festgelegt.)

$$
\leq\left(r \cdot d(g)^{-2 n(n+1) l}+(2 d(g)+r)^{\bar{\gamma}^{\prime}-1} r^{-(n+1) \kappa}\right) \cdot \text { const. }
$$

Wählt man zu gegebenem $n$ und $\bar{\gamma}$ (was wiederum $l$ und $\kappa$ festlegt) sowie zu $\beta>0$ nun

$$
r=d(g)^{q} \quad \text { mit } q>2 n(n+1) l+\beta
$$

und $\bar{\gamma}^{\prime}$ genügend groß, dann verschwindet der Ausdruck (5.5.15) schneller als $d(g)^{\beta}$. Wir haben also folgendes Ergebnis erhalten:

Satz 5.6. Es seien $\bar{\gamma}>0, \phi^{(0)}, \ldots, \phi^{(n)} \in \Phi_{\bar{\gamma}}$ und $\beta>0$ gegeben. Für $z u \bar{\gamma}$, $n$ und $\beta$ genügend großes $\bar{\gamma}^{\prime}>0$ und jede reguläre Projektion $p_{\bar{\gamma}^{\prime}}$ auf $\Phi_{\bar{\gamma}^{\prime}}$ gilt dann

$$
d(g)^{-\beta}\left\|\phi^{(0)}\left(g_{0}\right) \cdot \ldots \cdot \phi^{(n)}\left(g_{n}\right)-p_{\bar{\gamma}^{\prime}}\left(\phi^{(0)}\left(g_{0}\right) \cdot \ldots \cdot \phi^{(n)}\left(g_{n}\right)\right)\right\|_{E} \rightarrow 0 \quad \forall E>0
$$

im Limes scharf lokalisierter Testfunktionen $g_{j}$. 
Damit haben wir eine analoge Form der Produktentwicklung aus Satz 5.3 auch für beliebige Abstände etabliert, allerdings im Sinne von Distributionen. Die aus der Literatur bekannte Form der Produktentwicklung ergibt sich wiederum, indem man $p_{\bar{\gamma}^{\prime}}$ bezüglich einer Basis $\left\{\phi_{j}\right\}$ von $\Phi_{\bar{\gamma}^{\prime}}$ entwickelt; man erhält dann

$$
\phi^{(0 \ldots n)}(g) \approx \sum_{j} \sigma_{j}\left(\phi^{(0 \ldots n)}(g)\right) \phi_{j}=\sum_{j} c_{j}\left(g_{0}, \ldots, g_{n}\right) \phi_{j}
$$

Die Koeffizienten $c_{j}$ sind in diesem Fall temperierte Distributionen $\int$ in $g_{0}, \ldots, g_{n}$. Sie stimmen allerdings für raumartige Abstände mit den in Abschnitt 5.3 berechneten analytischen Funktionen überein, wie man durch Betrachtung der approximierenden Folgen lokaler Operatoren leicht sieht.

Auch die „allgemeine“ Operatorproduktentwicklung läßt sich auf das Tensorprodukt $\Phi_{\bar{\gamma}}^{\otimes n+1}$ erweitern, und sie weist analoge Symmetrieeigenschaften wie die raumartige Produktentwicklung auf. Die Argumentation kann aus Abschnitt 5.3 übernommen werden, wobei die Forderung nach „Verträglichkeit der Transformationen mit raumartigen Produkten" in Definition 5.4 entsprechend zu modifizieren ist. Wir gehen darauf hier aber nicht näher ein.

\section{A Funktionentheoretische Ergänzungen}

\section{A.1 Ein Phragmén-Lindelöf-Argument}

Aus dem Maximum-Prinzip der Funktionentheorie lassen sich Aussagen der folgenden Form gewinnen: Ist von einer Familie holomorpher Funktionen bekannt, daß sie gewissen Schranken genügt, die jedoch a priori an einigen Stellen des Holomorphiegebiets divergieren, dann ist die Familie tatsächlich gleichmäßig beschränkt. Ein Argument dieser Art benötigen wir in unserer Analyse von Operatorprodukten:

Lemma 5.7. $Z u k>0$ existiert eine Konstante $c>0$ mit der folgenden Eigenschaft: Ist $f$ holomorph in einer Umgebung eines Quadrats

$$
Q=\left\{z \in \mathbb{C} \mid \operatorname{Re} z \in\left[x_{0}-\delta, x_{0}+\delta\right] ; \operatorname{Im} z \in[-\delta, \delta]\right\} \quad\left(x_{0} \in \mathbb{R}, \delta>0 \text { fest }\right),
$$

und gilt die Abschätzung

$$
|f(z)| \leq c^{\prime}|\operatorname{Im} z|^{-k} \quad \text { für alle } z \in Q \text { mit einer Konstanten } c^{\prime}>0
$$

so folgt

$$
|f(z)| \leq c \cdot c^{\prime} \cdot \delta^{-k} \quad \text { für }\left|x_{0}-\operatorname{Re} z\right| \leq \frac{\delta}{2},|\operatorname{Im} z| \leq \frac{\delta}{2}
$$

Beweis. Ohne Einschränkung der Allgemeinheit können wir $x_{0}=0$ annehmen. Es sei

$$
a(z):=z^{k+1}
$$

\footnotetext{
${ }^{7}$ Die Temperiertheit folgt dabei wie in Satz 3.13 aus den polyomialen Energieschranken der Felder.
} 
Abbildung 5.1: Zum Beweis von Lemma 5.7

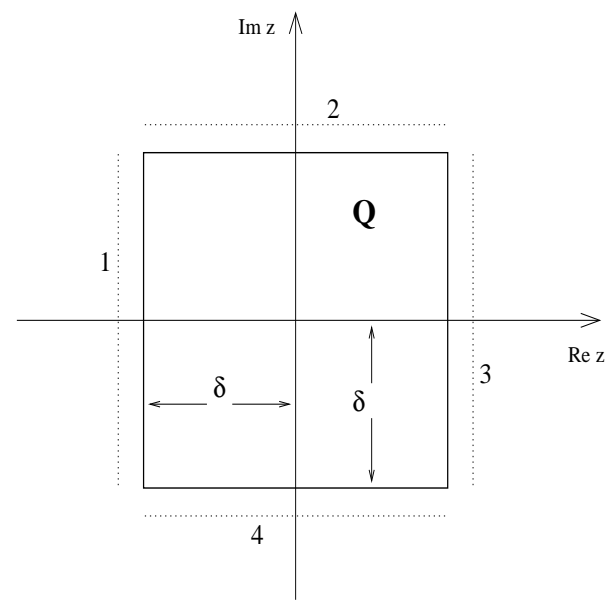

(wir wählen dabei einen Zweig mit Schnitt bei $z \in-\mathbb{R}^{+}$). Dann gilt

$$
|a(z)|=|z|^{k+1} \underset{z \rightarrow 0}{\longrightarrow} 0,
$$

also ist die Funktion nach $z=0$ stetig fortsetzbar (bei Annäherung aus ihrem Definitionsbereich heraus). Wir setzen weiter

$$
b(z):=a(\delta-z) a(\delta+z)
$$

diese Funktion ist holomorph im Inneren von $Q$ und stetig auf ganz $Q$. Dasselbe gilt für

$$
g(z):=f(z) b(z)
$$

Bekanntermaßen nimmt $|g(z)|$ sein Maximum in $Q$ auf dem Rand $\partial Q$ an. Dort läßt sich der Betrag von $g$ so abschätzen: Auf den Strecken 2 und 4 (vgl. Abbildung) gilt

$$
|g(z)|=|f(z)| \cdot|z-\delta|^{k+1} \cdot|z+\delta|^{k+1} \leq c^{\prime} \delta^{-k} \cdot(3 \delta)^{2 k+2}=c^{\prime} \cdot 3^{2 k+2} \delta^{k+2} .
$$

Auf Strecke 1, die wir als $z=-\delta+i t, t \in[-\delta, \delta]$ parametrisieren, hat man

$$
\begin{aligned}
|g(z)|=|f(-\delta+i t)| \cdot \mid & -2 \delta+\left.i t\right|^{k+1} \cdot|i t|^{k+1} \\
& \leq c^{\prime} \cdot|t|^{-k} \cdot|t|^{k+1}(3 \delta)^{k+1}=c^{\prime}|t|(3 \delta)^{k+1} \leq c^{\prime} \cdot 3^{k+1} \delta^{k+2}
\end{aligned}
$$

wobei diese Abschätzung aufgrund der Stetigkeit von $g$ auch bei $t=0$ richtig ist. Analoges erhält man auf Strecke 3. Insgesamt haben wir aus (5.A.5) und (5.A.6) also

$$
|g(z)| \leq c^{\prime} \cdot 3^{2 k+2} \delta^{k+2} \quad \forall z \in \partial Q
$$

und diese Schranke gilt folglich auch für alle $z \in Q$. Mithin ist für $z \in \frac{1}{2} \cdot Q$

$$
|f(z)| \leq \frac{|g(z)|}{|b(z)|} \leq \frac{c^{\prime} \cdot 3^{2 k+2} \delta^{k+2}}{\left(\frac{\delta}{2}\right)^{2 k+2}} \leq c \cdot c^{\prime} \cdot \delta^{-k} ;
$$

hierbei ist $c:=6^{2 k+2}$ ein rein numerischer Faktor. 


\section{A.2 Das Edge-of-the-Wedge-Theorem}

In unserer Analyse begegnen wir einem Fortsetzungsproblem für holomorphe Funktionen, das in der Wightman'schen Quantenfeldtheorie häufig anzutreffen ist. Wir formulieren zunächst die Fassung für Funktionen einer komplexen Variablen.

Satz 5.8. Es sei $\mathcal{E} \subset \mathbb{R}$ eine offene Menge und $f$ eine Funktion auf $(\mathbb{C} \backslash \mathbb{R}) \cup \mathcal{E}$, holomorph auf $\mathbb{C} \backslash \mathbb{R}$ und stetig an den reellen Punkten. Dann ist $f$ tatsächlich auf dem gesamten Definitionsbereich holomorph.

Der Beweis ist nicht schwer zu führen, etwa mit Hilfe des Satzes von Morera [FT.94, Kap. III, Satz 4.1].

Uns interessiert aber vor allem die Verallgemeinerung der Aussage auf Funktionen mehrerer komplexer Veränderlicher, die als Edge-of-the-Wedge-Theorem bezeichnet wird. In Satz 5.8 sind die beiden „Hälften" des Holomorphiegebiets durch die Bedingung $\pm \operatorname{Im} z>0$ abgegrenzt; dies wird in der mehrdimensionalen Fassung ersetzt durch $\pm \operatorname{Im} z \in \mathcal{C}$ mit einem konvexen Kegel $\mathcal{C}$. Der Rand des Holomorphiegebiets ist dann nicht mehr ein Teil der reellen Achse, sondern eine offene Menge im $\mathbb{R}^{n}$. Gegenüber dem eindimensionalen Fall ist besonders auffällig, daß das Fortsetzungsgebiet der „zusammengesetzten“ Funktion im mehrdimensionalen Fall über die ursprünglichen Holomorphiegebiete und ihren gemeinsamen Rand hinausgeht. Man erhält sogar einen sehr expliziten Ausdruck für die fortgesetzte Funktion.

Der untenstehende Beweis orientiert sich am Buch von Streater und Wightman [SW64, ch. 2-5]; er wird hier reproduziert, weil wir einige Zusatzaussagen über Schranken der fortgesetzten Funktionen benötigen, die üblicherweise nicht als Teil des Theorems genannt werden, sich aber aus den Details des Beweises ergeben.

Satz 5.9 (Edge-of-the-Wedge-Theorem). Es sei $\mathcal{E} \subset \mathbb{R}^{n}$ eine offene Menge, $\mathcal{C} \subset \mathbb{R}^{n}$ ein offener konvexer Kegel. Es seien $f_{+}, f_{-}$zwei holomorphe Funktionen auf $\mathcal{E}+i \mathcal{C}$ respektive $\mathcal{E}-i \mathcal{C}$; die Grenzwerte

$$
f_{0}(x):=\lim _{\mathcal{C} \ni y \rightarrow 0} f_{+}(x+i y)=\lim _{\mathcal{C} \ni y \rightarrow 0} f_{-}(x-i y) \quad(x \in \mathcal{E})
$$

mögen existieren und übereinstimmen; die Grenzfunktion $f_{0}(x)$ sei stetig auf $\mathcal{E}$ und bei Annäherung aus $\mathcal{E} \pm i \mathcal{C}$. Dann existiert eine komplexe Umgebung $\mathcal{O}$ von $\mathcal{E}$ und eine holomorphe Funktion $f$ auf $\mathcal{O}$, die auf $(\mathcal{E} \pm i \mathcal{C})$ mit $f_{ \pm}$und auf $\mathcal{E}$ mit $f_{0}$ übereinstimmt.

Das Gebiet $\mathcal{O}$ hängt hierbei nur von $\mathcal{E}$ und $\mathcal{C}$ ab, nicht aber von den $f_{ \pm}$. Außerdem verhält es sich kovariant unter reellen Translationen und Dilatationen, d.h. für $x_{0} \in \mathbb{R}^{n}, \lambda \in$ $\mathbb{R}^{+}$erhält man $z u\left(\lambda \mathcal{E}+x_{0}, \mathcal{C}\right)$ statt $(\mathcal{E}, \mathcal{C})$ das Holomorphiegebiet $\lambda \mathcal{O}+x_{0}$ statt $\mathcal{O}$.

Beweis. Zunächst bringen wir die geometrischen Objekte $\mathcal{C}, \mathcal{E}$ auf eine „Standardform“: Im konvexen Kegel $\mathcal{C}$ wählen wir $n$ linear unabhängige Vektoren $v_{j}$; die Linearkombinationen

$$
\sum_{j=1}^{n} \alpha_{j} v_{j} \quad \text { mit } \alpha_{j}>0
$$

bilden dann einen offenen Teilkegel $\mathcal{C}^{\prime}$ von $\mathcal{C}$, mit dem wir im folgenden arbeiten. Wir wählen eine lineare Abbildung $\mathbb{R}^{n} \rightarrow \mathbb{R}^{n}$, die die $v_{j}$ auf die Standardbasisvektoren $e_{j}$ des 
$\mathbb{R}^{n}$ abbildet und damit den Kegel $\mathcal{C}^{\prime}$ auf das Produkt der oberen Halbebenen. Die genannte Transformation erweitern wir auf $\mathbb{C}^{n} \rightarrow \mathbb{C}^{n}$; sie transformiert dann auch $\mathcal{E}$ innerhalb der reellen Ebene.

Der Satz macht Aussagen über lokale Eigenschaften der betrachteten Funktionen (in $\mathcal{E}$ ) - es reicht, diese in einer Umgebung jedes Punktes von $\mathcal{E}$ zu etablieren. Durch Anwendung reeller Translationen und reeller Dilatationen des $\mathbb{C}^{n}$ können wir einen gegebenen Punkt $x_{0} \in \mathcal{E}$ auf den Koordinatenursprung und eine gegebene Umgebung von $x_{0}$ auf eine Umgebung des Würfels $[-1,1]^{n} \subset \mathbb{R}^{n}$ transformieren. Diese Transformationen lassen $i \mathcal{C}^{\prime}$ invariant.

Kurz gesagt, wir können nach Anwendung der beschriebenen Transformationen annehmen, daß

$$
\begin{aligned}
& \mathcal{E} \supset[-1,1]^{n}, \\
& \mathcal{C}=\left\{\left(x_{1}, \ldots, x_{n}\right) \in \mathbb{R}^{n} \mid x_{j}>0 \forall j\right\}
\end{aligned}
$$

und müssen zeigen, daß sich eine Funktion $f$ mit den gewünschten Eigenschaften auf einer gewissen Nullumgebung finden läßt.

Es seien $K_{+}, K_{-}$die obere bzw. untere Hälfte des Einheitskreises in der $\mathbb{C}$-Ebene (mit positiver Orientierung). Wir definieren folgende Funktion von $n+1$ komplexen Variablen:

$$
\begin{aligned}
g\left(\zeta, z_{1}, \ldots, z_{n}\right):=\frac{1}{2 \pi i} \int_{K_{+}} \frac{f_{+}\left(\frac{u+z_{1}}{1+u z_{1}}, \ldots, \frac{u+z_{n}}{1+u z_{n}}\right)}{u-\zeta} d u & \\
& \quad+\frac{1}{2 \pi i} \int_{K_{-}} \frac{f_{-}\left(\frac{u+z_{1}}{1+u z_{1}}, \ldots, \frac{u+z_{n}}{1+u z_{n}}\right)}{u-\zeta} d u .
\end{aligned}
$$

Der Definitionsbereich umfaßt dabei $|\zeta|<1$ und $\left|z_{j}\right|<\frac{1}{2}$; die Integralausdrücke sind dann wohldefiniert, denn man berechnet leicht, daß

$$
\operatorname{Im}\left(\frac{u+z_{j}}{1+u z_{j}}\right)=\frac{\left(1-\left|z_{j}\right|^{2}\right) \operatorname{Im} u+\left(1-|u|^{2}\right) \operatorname{Im} z_{j}}{\left|1+u z_{j}\right|^{2}} \underset{\left(u \in K_{ \pm}\right)}{=} \frac{1-\left|z_{j}\right|^{2}}{\left|1+u z_{j}\right|^{2}} \operatorname{Im} u
$$

was für $u \in K_{+}$positiv, für $u \in K_{-}$negativ ist; für $u= \pm 1$ erhält man $\frac{u+z_{j}}{1+u z_{j}}= \pm 1$, also zulässige Randwerte des Definitionsbereichs von $f_{ \pm}$.

Die in (5.A.11) definierte Funktion $g$ ist (wegen der Stetigkeit der $f_{ \pm}$auch an den Randwerten) in ihren $n+1$ Variablen stetig; in der Variablen $\zeta$ ist sie holomorph. Nehmen wir zusätzlich an, daß die partiellen Ableitungen $\frac{\partial}{\partial z_{j}} f_{ \pm}$in einer Umgebung der Punkte $\pm(1, \ldots, 1)$ beschränkt sind (zu dieser Annahme siehe unten), dann ist auch $g$ partiell nach $z_{j}$ differenzierbar (durch Vertauschung von Integration und Differentiation). Damit ist $g$ im Bereich $|\zeta|<1,\left|z_{j}\right|<\frac{1}{2}$ holomorph in allen Variablen. Wir werden im folgenden zeigen, daß die dann für $\left|z_{j}\right|<\frac{1}{2}$ holomorphe Funktion

$$
f\left(z_{1}, \ldots, z_{n}\right):=g\left(0, z_{1}, \ldots, z_{n}\right)
$$

die gewünschte Fortsetzung liefert, d.h. daß sie auf dem jeweiligen gemeinsamen Definitionsbereich mit den $f_{ \pm}(z)$ übereinstimmt. 
Um dies zu sehen, betrachten wir $g$ auf einer reellen Nullumgebung $z_{j} \in \mathbb{R},\left|z_{j}\right|<\frac{1}{2}$. Die Nenner der Integranden in (5.A.11), nämlich

$$
f_{ \pm}^{1}(u):=f_{ \pm}\left(\frac{u+z_{1}}{1+u z_{1}}, \ldots, \frac{u+z_{n}}{1+u z_{n}}\right)
$$

sind dann holomorphe Funktionen von $u$ (jeweils für $\pm \operatorname{Im} u>0,|u|<1+\epsilon, \epsilon>0$ geeignet) mit gemeinsamer stetiger Fortsetzung nach $\operatorname{Im} u=0$. Wir können daher Satz 5.8 anwenden, der besagt, daß $f_{+}^{1}(u)$ und $f_{-}^{1}(u)$ Einschränkungen einer einzigen holomorphen Funktion $f^{1}(u)$ sind. Damit wird (5.A.11) zur Cauchy-Integralformel für $f^{1}$; man erhält:

$$
\begin{aligned}
& g\left(\zeta, z_{1}, \ldots, z_{n}\right)=f^{1}(\zeta)=f_{ \pm}\left(\frac{\zeta+z_{1}}{1+\zeta z_{1}}, \ldots, \frac{\zeta+z_{n}}{1+\zeta z_{n}}\right) \\
& \quad \text { für } z_{j} \in \mathbb{R},\left|z_{j}\right|<\frac{1}{2}, \pm \operatorname{Im} \zeta>0 .
\end{aligned}
$$

Betrachten wir dies bei festem $\zeta$, so folgt, daß die holomorphen Funktionen $g(\zeta, \cdot)$ und $f_{ \pm}\left(\frac{\zeta+z_{1}}{1+\zeta z_{1}}, \ldots\right)$ auf einer reellen offenen Menge übereinstimmen (wobei \pm je nach Vorzeichen von $\operatorname{Im} \zeta \mathrm{zu}$ wählen ist). Damit stimmen sie aber auf ihrem gesamten Definitionsbereich überein. Gehen wir zum Grenzwert $\zeta \rightarrow 0$ über, dann bedeutet dies

$$
g\left(0, z_{1}, \ldots, z_{n}\right)=f_{ \pm}\left(z_{1}, \ldots, z_{n}\right) \quad \text { für } \operatorname{Im} z \in \pm \mathcal{C},\left|z_{j}\right|<\frac{1}{2} .
$$

Damit ist die gewünschte analytische Fortsetzung der Funktionen $f_{ \pm}$gefunden.

Wir müssen im allgemeinen Fall nun noch die erhaltene Fortsetzung und deren Holomorphiegebiet auf die ursprüngliche geometrische Situation „zurücktransformieren“, d.h. die oben beschriebenen Translationen, Dilatationen und linearen Transformationen des Kegels rückgängig machen. Das ist offenbar in der gewünschten Weise möglich, indem man das Argument von $g(0, \cdot)$ transformiert; die behauptete Kovarianz des Gebiets $\mathcal{E}$ unter reellen Translationen und Dilatationen ist klar.

$\mathrm{Zu}$ behandeln bleibt noch der Fall, daß die partiellen Ableitungen $\frac{\partial}{\partial z_{j}} f_{ \pm}$in einer Umgebung der Punkte $\pm(1, \ldots, 1)$ nicht a priori beschränkt sind. In diesem Fall untersuchen wir zunächst die Stammfunktionen

$$
F_{ \pm}\left(z_{1}, \ldots, z_{n}\right):=\int_{-1}^{z_{1}} d \zeta_{1} \ldots \int_{-1}^{z_{n}} d \zeta_{n} f_{ \pm}\left(\zeta_{1}, \ldots, \zeta_{n}\right)
$$

Sie erfüllen ebenfalls alle Voraussetzungen des Satzes, und ihre partiellen Ableitungen sind in einer Umgebung der angegebenen Punkte beschränkt. Mit obiger Argumentation erhalten wir eine gemeinsame holomorphe Fortsetzung der $F_{ \pm}$, und damit auch ihrer Ableitungen $f_{ \pm}$, in eine komplexe Umgebung von $\mathcal{E}$. Also müssen die partiellen Ableitungen der $f_{ \pm}$tatsächlich lokal gleichmäßig beschränkt sein (auch an den reellen Punkten), und wir können die oben durchgeführte Argumentation auch auf die $f_{ \pm}$anwenden.

Wie angekündigt, können wir nun noch bestimmte Schranken an die Funktionen $f_{ \pm}$auf die Fortsetzung $f$ (auf ihrem gesamten Definitionsbereich) übertragen. Für die fortgesetzte Funktion $f=g(0, \cdot)$ erhält man aus Gleichung (5.A.11) die Abschätzung

$$
|f(z)| \leq \sup _{u \in K_{ \pm}}\left|f_{ \pm}\left(\frac{u+z_{1}}{1+u z_{1}}, \ldots, \frac{u+z_{n}}{1+u z_{n}}\right)\right| \quad \text { für }\left|z_{j}\right|<\frac{1}{2} .
$$


Aus (5.A.12) entnimmt man, daß im angegebenen Bereich

$$
\left|\operatorname{Im}\left(\frac{u+z_{j}}{1+u z_{j}}\right)\right|=\frac{\left(1-\left|z_{j}\right|^{2}\right)|\operatorname{Im} u|}{\left|1+u z_{j}\right|^{2}} \leq 4 ;
$$

damit können wir (5.A.18) ersetzen durch

$$
|f(z)| \leq \sup _{ \pm}\left\{\left|f_{ \pm}(x \pm i y)\right| \mid x \in \mathcal{E}, y \in \mathcal{C},\|y\| \leq 4 n\right\}
$$

Diese Abschätzungen gelten für die in (5.A.10) beschriebene ,geometrische Standardsituation“. Die Übertragung auf allgemeinere Gebiete ist vergleichsweise unübersichtlich; wir beschränken uns auf folgenden Fall: Es sei $\mathcal{C}$ ein offener konvexer Kegel, den wir im folgenden festhalten, und $\mathcal{E}$ eine Kugel (Radius $r$, Mittelpunkt $x_{0}$ ). Zunächst sei $r=1$, $x_{0}=0$; wir erhalten dann eine holomorph fortgesetzte Funktion mindestens auf einer komplexen Kugel mit einem gewissen festen Radius $r_{0}>0 \mathrm{um}$ den Ursprung. Genügen die $f_{ \pm}$ Abschätzungen der Form

$$
\left|f_{ \pm}(x \pm i y)\right| \leq g(\|y\|) \quad(x \in \mathcal{E}, y \in \mathcal{C})
$$

mit einer monoton wachsenden Funktion $g$, dann erhalten wir aus (5.A.20) die Ungleichung

$$
|f(z)| \leq g\left(r_{1}\right)
$$

auf der besagten Kugel; $r_{1}=4 n$ ist eine Konstante, die nicht von $f$ oder $g$ abhängt. Nun können wir die reelle Kugel $\mathcal{E}$ skalieren und translatieren, wobei $\mathcal{C}$ fest bleibt; unser Ergebnis ist dann folgendes:

Korollar 5.10. Es sei $\mathcal{C} \subset \mathbb{R}^{n}$ ein offener konvexer Kegel. Es gibt Konstanten $r_{0}, r_{1}>0$ mit folgender Eigenschaft: Sind $f_{ \pm}$zwei Funktionen, die den Voraussetzungen von Satz 5.9 genügen, wobei $\mathcal{C}$ wie oben und $\mathcal{E} \subset \mathbb{R}^{n}$ eine Kugel mit Radius $r$ und Mittelpunkt $x_{0}$ ist, dann umfaßt das Gebiet $\mathcal{O}$ mindestens die komplexe Kugel $\left|x_{0}-z\right| \leq r \cdot r_{0}$. Genügen die $f_{ \pm}$zusätzlich Abschätzungen der Form

$$
\left|f_{ \pm}(x+i y)\right| \leq g(\|y\|) \quad(x \in \mathcal{E}, y \in \mathcal{C})
$$

mit einer monoton wachsenden Funktion $g$, dann gilt für die fortgesetzte Funktion $f$ :

$$
|f(z)| \leq g\left(r_{1} \cdot r\right) \quad \text { für }\left\|x_{0}-z\right\|<r \cdot r_{0} .
$$





\section{Kapitel 6}

\section{Normalprodukte}

In wechselwirkenden Quantenfeldtheorien erwartet man, daß die Zeitentwicklung der Quantenfelder durch nichtlineare Feldgleichungen bestimmt wird. Um diese zu formulieren, benötigt man einen Produktbegriff für Felder am gleichen Raum-Zeit-Punkt. Eine naive Definition dieses Produkts führt jedoch, wie wir gesehen hatten, zu Divergenzen. Um ein endliches Ergebnis zu erhalten, muß man im Limes dicht benachbarter Punkte gewisse Anteile des Produkts subtrahieren; auf diese Weise läßt sich die Existenz der Feldgleichungen zumindest störungstheoretisch begründen [Val54, Fed61, Bra67, Bra70, Zim67].

Eine systematische Behandlung dieser „Subtraktionsterme“ ist mit der Operatorproduktentwicklung möglich [Zim70, Low70] : Aus den eventuell divergenten Termen der Entwicklung wählt man einen aus, der dann als (Zimmermann-)Normalprodukt der Felder bezeichnet wird. Dies kann man als Verallgemeinerung der Situation in der freien Feldtheorie verstehen, wo das Wickprodukt als Limes eines raumartigen Produkts nach Subtraktion gewisser Vakuumerwartungswerte erhalten werden kann (Wick'sches Theorem).

Wir verwenden hier die raumartige Operatorproduktentwicklung, um im Kurzabstandslimes ein Normalprodukt von Feldern zu definieren. Dieses Normalprodukt existiert als Vektorraum - es enthält alle Terme der Entwicklung, die im Limes „relevant“ bleiben. Die Auswahl eines einzelnen Terms als das Normalprodukt eines Ausdrucks (als Analogon zum Wickprodukt) ist im allgemeinen nicht eindeutig möglich - man wird dies auch nicht erwarten -, kann aber durch gewisse Kriterien eingeschränkt werden.

Wir diskutieren dann das Verhalten dieses Normalprodukt-Vektorraums unter Symmetrietransformationen und bei Differentiation. Weiter geben wir ein Verfahren an, um in einem gegeben quantenfeldtheoretischen Modell den "Inhalt" an linearen und nichtlinearen Feldgleichungen zu bestimmen. 


\subsection{Das Konzept „Normalprodukt“"}

Die Definition eines Produkts von Quantenfeldern am selben Raum-Zeit-Punkt bedarf einiger technischer Anstrengungen. In Kapitel 5 hatten wir untersucht, inwieweit Produkte von Feldern an verschiedenen Raum-Zeit-Punkten

$$
\phi^{(0 \ldots n)}(x)=\phi^{(0)}\left(x_{0}\right) \cdot \ldots \cdot \phi^{(n)}\left(x_{n}\right)
$$

definiert sind - für raumartig getrennte $x_{0}, \ldots, x_{n}$ existiert das Produkt z.B. als quadratische Form. Geht man jedoch zum Limes koinzidierender Punkte über, etwa $x_{j} \rightarrow 0$, dann hat das Produkt (6.1.1) im allgemeinen keinen endlichen Grenzwert; schon in der freien Feldtheorie treten hier divergente Terme auf.

Allerdings existiert in der freien Theorie mit der Wick-Ordnung ein Verfahren, um trotzdem ein Normalprodukt (Wickprodukt) für koinzidierende Punkte eindeutig festlegen zu können: Man subtrahiert vom raumartigen Produkt gewisse „Gegenterme“, die sich aus Vakuumerwartungswerten der Felder ergeben, und erhält so einen endlichen Grenzwert. Für ein reelles skalares freies Feld $\phi$ betrachtet man etwa

$$
: \phi\left(x_{0}\right) \ldots \phi\left(x_{n}\right):=\sum_{l=0}^{\left[\frac{n+1}{2}\right]}(-1)^{l} \sum_{C_{l}}\left(\Omega\left|\phi\left(x_{j_{1}}\right) \ldots \phi\left(x_{j_{2 l}}\right)\right| \Omega\right) \cdot \phi\left(x_{k_{1}}\right) \ldots \phi\left(x_{k_{n+1-2 l}}\right),
$$

was im Limes $x_{j} \rightarrow 0$ konvergent ist [W(665] . (Die Summe $\sum_{C_{l}}$ läuft über alle Partitionen $\left(j_{1} \ldots j_{2 l}\right)\left(k_{1} \ldots k_{n+1-2 l}\right)$ der Indizes $\left.(0 \ldots n).\right)$ Im einfachsten Fall eines zweistelligen Produkts erhält man so etwas expliziter

$$
\phi\left(x_{0}\right) \phi\left(x_{1}\right)-\left(\Omega\left|\phi\left(x_{0}\right) \phi\left(x_{1}\right)\right| \Omega\right) \underset{x_{0}, x_{1} \rightarrow 0}{\longrightarrow}: \phi^{2}:(0)
$$

wobei das Wickprodukt : $\phi^{2}$ : ein reguläres Wightman-Feld ist.

Diese Konvergenzeigenschaften beruhen allerdings auf der speziellen Struktur der freien Feldtheorie (etwa der Eigenschaften der Erzeugungs- und Vernichtungsoperatoren) und lassen sich nicht direkt verallgemeinern. Trotzdem kann die Idee des Abziehens geeigneter divergenter Gegenterme verwendet werden, um störungstheoretisch eine "renormierte" Version von nichtlinearen Feldgleichungen („finite local field equations“) zu betrachten, etwa in der Quantenelektrodynamik [Bra67, Bra70] oder der $\phi^{4}$-Theorie [Zim67], oder um in zweidimensionalen Modellen Ströme zu definieren [.Joh61, Som63].

Eine systematische Möglichkeit zur Behandlung solcher Subtraktionsmethoden bietet die Operatorproduktentwicklung: Schreibt man sie für das Produkt $\phi^{(0 \ldots n)}(x)$ in einer Basis aus, dann lautet sie grob

$$
\phi^{(0 \ldots n)}(x) \approx \sum_{j=1}^{J} c_{j}(x) \phi_{j} \quad \text { für } x \underset{\mathrm{R}}{\longrightarrow} 0,
$$

und man erwartet daher, daß etwa unter geeigneten Voraussetzungen an den Koeffizienten $c_{J}(x)$ gilt

$$
\phi_{J}=\lim _{x \underset{\mathrm{R}}{\longrightarrow} 0}\left(\frac{1}{c_{J}(x)} \phi^{(0 \ldots n)}(x)-\sum_{j=1}^{J-1} \frac{c_{j}(x)}{c_{J}(x)} \phi_{j}\right) .
$$


In Analogie円 zu (6.1.2) bzw. (6.1.3) kann man $\phi_{J}$ dann als verallgemeinertes Wickprodukt oder Normalprodukt von $\phi^{(0 \ldots n)}$ bezeichnen, wie dies zuerst von Zimmermann [Zim70] in der Störungstheorie vorgeschlagen wurde. Allerdings ist die Auswahl von $\phi_{J}$ in (6.1.5) im allgemeinen keineswegs eindeutig - man könnte auch ein anderes der Felder $\phi_{j}$ wählen, solange der zugehörige Koeffizient $c_{j}(x)$ im Limes „relevant" bleibt (nicht verschwindet). Diese Zimmermann-Normalprodukte wurden störungstheoretisch [Low71] und in zweidimensionalen exakt lösbaren Theorien [Low70, LS71] betrachtet; man kann hier in gegebenen Modellen die Auswahl des Normalprodukts konkretisieren. Das Ergebnis ist auch insofern eine Verallgemeinerung des Wick-Produkts, als es einige von dessen Eigenschaften (Kommutativität, Lorentz-Kovarianz) ebenfalls aufweist. Normalprodukte der beschriebenen Art spielen auch in der Renormierungsanalyse mit Flußgleichungen eine Rolle [KK.92, KK.93].

Ein anderer, axiomatischer Ansatz zur Definition von Normalprodukten auf der Grundlage der Operatorproduktentwicklung stammt von Baumann [Bau75] auf der Grundlage der Ergebnisse von Schlieder und Seiler [SSe73]. Hier wird das Normalprodukt nicht durch Subtraktion von Termen im raumartigen Limes definiert, sondern durch Multiplikation des raumartigen Produkts mit einem geeigneten $c$-Zahl-Faktor, so daß der resultierende Ausdruck auf einer Nullumgebung holomorph ist. Diese Methode läßt sich allerdings nur in speziellen Theorien anwenden (etwa für ein masseloses, nicht aber für ein massives freies Feld); sie scheint sich nicht auf allgemeinere Fälle zu übertragen.

\subsection{Definition von Normalprodukten}

Zur Definition eines Normalprodukts von Felder in unserem Rahmen gehen wir von der raumartigen Operatorproduktentwicklung aus, die in Abschnitt 5.3 etabliert wurde. Es seien $\bar{\gamma}>0, n \in \mathbb{N}$ fest; wir werden das Normalprodukt von $\Pi \in \Phi_{\bar{\gamma}}^{\otimes n+1}$ definieren. (Es empfiehlt sich hier, im Hinblick auf die spätere Anwendung von Differentialoperatoren auch Linearkombinationen von Produkten zuzulassen.) Für genügend großes $\bar{\gamma}^{\prime}>0$ wissen wir, daß

$$
\left\|\Pi(x)-p_{\bar{\gamma}^{\prime}} \Pi(x)\right\|_{E \underset{\mathrm{R}}{\longrightarrow} 0} 0 \quad \forall E>0,
$$

wenn $p_{\bar{\gamma}^{\prime}}$ eine reguläre Projektion auf $\Phi_{\bar{\gamma}^{\prime}}$ ist. Der Raum $\Phi_{\bar{\gamma}^{\prime}}$ ist also „ausreichend groß“, um $\Pi(x)$ im Limes zu approximieren. Wir versuchen nun, diesen Raum zu „minimieren“, d.h. Richtungen in $\Phi_{\bar{\gamma}^{\prime}}$ auszuschließen, die im betrachteten Limes nicht zur Approximation beitragen. Elemente des „minimierten“ Raums sind dann Kandidaten für das Normalprodukt des Ausdrucks П. Um dies zu präzisieren, führen wir folgenden Begriff ein:

Definition 6.1. Sei $\Pi \in \Phi_{\bar{\gamma}}^{\otimes n+1}$ und $V \subset \bar{\Sigma}^{*}$ ein endlichdimensionaler Unterraum. $V$ heißt raumartig approximierend für $\Pi$, wenn es eine reguläre Projektion $p_{V}$ auf $V$ gibt, so

\footnotetext{
1 Es sei bemerkt, daß im betrachteten Beispiel die aus der Operatorproduktentwicklung abgeleitete Approximation (6.1.5) nur im einfachen Fall des Wick-Quadrats mit dem Ausdruck aus dem Wick'schen Theorem (6.1.2) wörtlich übereinstimmt. Bei Produkten höherer Ordnung sind die Terme für endliche raumartige Abstände unterschiedlich, da in der hier beschriebenen Produktentwicklung lediglich die Koeffizienten (nicht die Felder) ortsabhängig sind, im Gegensatz zum Zugang über die Wick-Ordnung. Der Unterschied verschwindet aber asymptotisch.
} 
$d a \beta$

$$
\left\|\Pi(x)-p_{V} \Pi(x)\right\|_{E} \underset{\mathrm{R}}{\longrightarrow} 0 \quad \forall E>0 .
$$

Wir können also stets $\bar{\gamma}^{\prime}>0$ finden, so daß $\Phi_{\bar{\gamma}^{\prime}}$ raumartig approximierend für $\Pi$ ist. Die Wahl des Projektors $p_{V}$ in der Definition ist nicht wesentlich: Sei $p_{V}^{\prime}$ eine weitere reguläre Projektion auf $V$, dann folgt aus der in der Definition genannten Approximationseigenschaft wegen der Energiebeschränktheit von $p_{V}^{\prime}$ sofort

$$
\left\|p_{V}^{\prime} \Pi(x)-p_{V}^{\prime} p_{V} \Pi(x)\right\|_{E} \underset{\mathrm{R}}{\longrightarrow} 0 \quad \forall E>0 .
$$

Wegen $p_{V}^{\prime}\left\lceil V=\mathrm{id}_{V}\right.$ gilt aber $p_{V}^{\prime} p_{V}=p_{V}$, weshalb

$$
\left\|p_{V}^{\prime} \Pi(x)-p_{V} \Pi(x)\right\|_{E} \underset{\mathrm{R}}{\longrightarrow} 0 \quad \forall E>0 .
$$

Zusammen mit dem in der Definition geforderten Grenzwertverhalten ergibt sich also

$$
\left\|\Pi(x)-p_{V}^{\prime} \Pi(x)\right\|_{E} \underset{\mathrm{R}}{\longrightarrow} 0 \quad \forall E>0,
$$

d.h. die Approximationseigenschaft in der Definition gilt tatsächlich für jede reguläre Projektion $p_{V}$ auf $V$.

Der nun folgende Satz ist zentral für die Definition eines „minimierten approximierenden Raums".

Satz 6.2. Sind zwei Räume $V, V^{\prime}$ für $\Pi$ raumartig approximierend, dann ist es auch $V \cap V^{\prime}$.

Beweis. Wir schreiben $V_{\cap}=V \cap V^{\prime}$ und $V_{\cup}=\operatorname{Span}\left(V \cup V^{\prime}\right)$. Weiter wählen wir eine Zerlegung

$$
V_{\cup}=V_{\cap} \oplus V_{1} \oplus V_{1}^{\prime}, \quad \text { wobei } \quad V=V_{\cap} \oplus V_{1}, \quad V^{\prime}=V_{\cap} \oplus V_{1}^{\prime},
$$

und eine reguläre Projektion $p_{\cup}$ auf $V_{\cup}$. Dann sind

$$
p_{V}:=(\mathbf{1} \oplus \mathbf{1} \oplus 0) \circ p_{\cup}, \quad p_{V^{\prime}}:=(\mathbf{1} \oplus 0 \oplus \mathbf{1}) \circ p_{\cup}
$$

reguläre Projektionen auf $V$ respektive $V^{\prime}$, und

$$
p_{\cap}:=p_{V} \circ p_{V^{\prime}}=(\mathbf{1} \oplus 0 \oplus 0) \circ p_{\cup}
$$

ist eine reguläre Projektion auf $V_{n}$. Man hat nun

$$
\begin{aligned}
&\left\|\Pi(x)-p_{\cap} \Pi(x)\right\|_{E}=\left\|\Pi(x)-p_{V} p_{V^{\prime}} \Pi(x)\right\|_{E} \\
& \leq\left\|\Pi(x)-p_{V} \Pi(x)\right\|_{E}+\left\|p_{V}\left(\Pi(x)-p_{V^{\prime}} \Pi(x)\right)\right\|_{E} \\
& \leq\left\|\Pi(x)-p_{V} \Pi(x)\right\|_{E}+\left\|p_{V}\right\|_{E}\left\|\left(\Pi(x)-p_{V^{\prime}} \Pi(x)\right)\right\|_{E^{\prime}} \underset{\mathrm{R}}{\longrightarrow} 0 \quad \forall E>0
\end{aligned}
$$

wobei $E^{\prime}$ durch $p_{V}$ bestimmt wird. Also ist auch $V_{\cap}$ raumartig approximierend für $\Pi$. 
Mit Hilfe dieses Ergebnisses können wir nun leicht einen „minimierten approximierenden Raum" definieren: Wir setzen

$$
\mathrm{N}[\Pi]:=\bigcap_{V} V
$$

wobei $V$ alle für $\Pi$ raumartig approximierenden Räume durchläuft. (Da es sich hier um endlichdimensionale Vektorräume handelt, reicht es aus, endliche Durchschnitte zu betrachten.) Wir nennen $\mathrm{N}[\Pi]$ den Normalproduktraum von $\Pi$. Seine offensichtlichen Eigenschaften fassen wir kurz zusammen:

Satz 6.3. Für den Normalproduktraum N[П] gilt:

- $\mathrm{N}[\Pi]$ ist raumartig approximierend für $\Pi$.

- Ist ein Raum $V \subset \bar{\Sigma}^{*}$ für $\Pi$ raumartig approximierend, dann gilt $\mathrm{N}[\Pi] \subset V$.

- $Z u \bar{\gamma}>0, n \in \mathbb{N}_{0}$ gibt es ein $\bar{\gamma}^{\prime}>0$, so daß $\mathrm{N}[\Pi] \subset \Phi_{\bar{\gamma}^{\prime}}$ für alle $\Pi \in \Phi_{\bar{\gamma}}^{\otimes n+1}$.

- Geht $\Pi^{\prime}$ aus $\Pi$ durch Permutation der Tensorfaktoren hervor, so ist $\mathrm{N}[\Pi]=\mathrm{N}\left[\Pi^{\prime}\right]$.

Dabei folgt die zuletzt genannte Eigenschaften aus der Kommutativität des raumartigen Produkts. Auch der Normalproduktraum ist also „kommutativ“.

Für die Elemente von N[I] können wir Zimmermanns Approximationsformel (6.1.5) etablieren: Wir wählen eine reguläre Projektion $p$ auf $\mathrm{N}[\Pi]$. Ein fest gewähltes $\phi \in \mathrm{N}[\Pi]$ ergänzen wir durch Vektoren $\left\{\phi_{1}, \ldots, \phi_{J}\right\}$ zu einer Basis von $\mathrm{N}[\Pi]$ und schreiben $p$ bezüglich dieser Basis aus:

$$
p=\sigma \phi+\sum_{j=1}^{J} \sigma_{j} \phi_{j}
$$

respektive

$$
p \Pi(x)=c(x) \phi+\sum_{j=1}^{J} c_{j}(x) \phi_{j}, \quad \text { wobei } \quad c(x)=\sigma(\Pi(x)), c_{j}(x)=\sigma_{j}(\Pi(x)) .
$$

Dabei verschwindet $c(x)$ im raumartigen Limes nicht, denn sonst hätte man

$$
\left\|\Pi(x)-\sum_{j=1}^{J} \sigma_{j}(\Pi(x)) \phi_{j}\right\|_{E} \leq\|\Pi(x)-p \Pi(x)\|_{E}+c(x)\|\phi\|_{E} \underset{\mathrm{R}}{\longrightarrow} 0
$$

also wäre auch Span $\left\{\phi_{j}\right\}$ raumartig approximierend für $\Pi$, im Widerspruch zur Minimalität von $\mathrm{N}[\Pi]$. Auf einer gewissen raumartigen Folge $\left(x_{k}\right)$ gilt also $c(x) \geq \bar{c}$ mit einer Konstanten $\bar{c}>0$. Damit folgt aus

$$
\|\Pi(x)-p \Pi(x)\|=\left\|\Pi(x)-\sum_{j=1}^{J} c_{j}(x) \phi_{j}-c(x) \phi\right\|_{E} \underset{\mathrm{R}}{\longrightarrow} 0
$$


durch Division auch

$$
\left\|\phi-\frac{1}{c(x)} \Pi(x)-\sum_{j=1}^{J} \frac{c_{j}(x)}{c(x)} \phi_{j}\right\|_{E} \underset{\mathrm{R}}{\stackrel{\left(x_{k}\right)}{\longrightarrow}} 0
$$

also

$$
\phi=\lim _{x_{k} \rightarrow 0}\left(\frac{1}{c(x)} \Pi(x)-\sum_{j=1}^{J} \frac{c_{j}(x)}{c(x)} \phi_{j}\right)
$$

im Sinne von Linearformen auf $\bar{\Sigma}$.

Um die Konsistenz des Schemas zu überprüfen, berechnen wir noch den Normalproduktraum für $n=0$, d.h. für „Produkte“ mit nur einem Faktor. Dazu sei $\phi \in \Phi_{\mathrm{FH}}, \phi \neq 0$, und wir wählen $\sigma \in \bar{\Sigma}$ mit $\sigma(\phi)=1$, so daß $\sigma(\cdot) \phi$ eine reguläre Projektion auf $\mathbb{C} \phi$ ist. Dann haben wir

$$
\|\phi(x)-\sigma(\phi(x)) \phi\|_{E} \leq\|\phi(x)-\phi\|_{E}+|1-\sigma(\phi(x))|\|\phi\|_{E} \underset{\mathrm{R}}{\longrightarrow} 0,
$$

da $P(E) \phi(x) P(E)$ normstetig und $\sigma$ energiebeschränkt ist. Folglich ist $\mathbb{C} \phi$ raumartig approximierend für $\phi$. Offenbar läßt sich dieser approximierende Raum nicht weiter verkleinern (der Nullraum ist nicht raumartig approximierend), so daß wir erhalten:

$$
\mathrm{N}[\phi]=\mathbb{C} \phi .
$$

Weiter untersuchen wir die Symmetrieeigenschaften des Normalproduktraums. Dazu sei $\alpha$ eine lokale Transformation und verträglich mit raumartigen Produkten. $\Pi \in \Phi_{\bar{\gamma}}^{\otimes n+1}$ sei fest, $V \subset \bar{\Sigma}^{*}$ für $\Pi$ raumartig approximierend und $p_{V}$ eine reguläre Projektion auf $V$. Mit

$$
\left\|\Pi(x)-p_{V} \Pi(x)\right\|_{E} \underset{\mathrm{R}}{\longrightarrow} 0 \quad \forall E>0
$$

gilt auch

$$
\left\|\alpha \Pi(x)-\alpha p_{V} \Pi(x)\right\|_{E} \underset{\mathrm{R}}{\longrightarrow} 0 \quad \forall E>0 .
$$

Da aber $\alpha(\Pi(x))=(\alpha \Pi)(\alpha . x)$ nach Lemma 5.5, haben wir

$$
\left\|(\alpha \Pi)(\alpha . x)-\left(\alpha p_{V} \alpha^{-1}\right)(\alpha \Pi)(\alpha . x)\right\|_{E \underset{\mathrm{R}}{\longrightarrow} 0} 0 \quad \forall E>0 .
$$

Dabei ist $\alpha p_{V} \alpha^{-1}$ eine reguläre Projektion auf $\alpha V$, analog zu Satz 4.6. Nach Definition 5.4 ist $x \underset{\mathrm{R}}{\longrightarrow} 0$ äquivalent zu $\alpha . x \underset{\mathrm{R}}{\longrightarrow} 0$; damit wird also $\alpha V$ raumartig approximierend für $\alpha \Pi$. Dies gilt insbesondere für $V=\mathrm{N}[\Pi]$, so daß wir

$$
\mathrm{N}[\alpha \Pi] \subset \alpha \mathrm{N}[\Pi]
$$

erhalten. Eine analoge Argumentation mit $\alpha^{-1}$ statt $\alpha$ liefert die umgekehrte Inklusion; man hat also

$$
\mathrm{N}[\alpha \Pi]=\alpha \mathrm{N}[\Pi]
$$


für $\Pi \in \Phi_{\bar{\gamma}}^{\otimes n+1}$ und lokale Transformationen $\alpha$, die verträglich mit raumartigen Produkten sind.

Der Normalproduktraum $\mathrm{N}[\Pi]$ als Vektorraum erfüllt damit die Eigenschaften, die man von einer Verallgemeinerung des Wickprodukts erwartet. Für das Quadrat eines reellen skalaren freien Feldes hat man beispielsweise

$$
\mathrm{N}[\phi \otimes \phi]=\operatorname{Span}\left\{\mathbf{1},: \phi^{2}:\right\},
$$

wie in Abschnitt 7.5 gezeigt wird.

Man beachte, daß N[ח] mehr Informationen enthält als nur die Angabe eines Teilraums von $\Phi_{\mathrm{FH}}$ : Da wir wissen, daß

$$
\left\|\Pi(x)-p_{\mathrm{N}[\Pi]} \Pi(x)\right\|_{E} \underset{\mathrm{R}}{\longrightarrow} 0 \quad \forall E>0
$$

für reguläre Projektionen $p_{\mathrm{N}[\Pi]}$ auf $\mathrm{N}[\Pi]$, kennen wir auch die „Approximationsterme“ $p_{\mathrm{N}[\Pi]} \Pi(x)$ bis auf Anteile, die im Limes verschwinden und die von der Auswahl der Projektion abhängen können.

Offen bleibt aber im allgemeinen Fall die Frage, wie und ob sich ein einzelner Vektor aus diesem Raum als das Normalprodukt von $\Pi$ auszeichnen läßt, ähnlich dem Wickprodukt in der freien Feldtheorie. Aus den Erfahrungen in zweidimensionalen Modellen wird man im allgemeinen nicht erwarten, daß sich eine solche Wahl eindeutig treffen läßt [Som63]. Abhängig von der konkret gegebenen Situation kann man aber einige „Auswahlkriterien“ angeben, die die Wahlfreiheit für das Normalprodukt zumindest eingrenzen:

Referenzzustände Die Auswahl eines Vektors bzw. eines eindimensionalen Unterraums in $\mathrm{N}[\Pi]$ kann eingeschränkt werden, indem man verlangt, daß sein Erwartungswert in gewissen ausgezeichneten „Referenzzuständen“ (etwa im Vakuum) verschwindet. Dies entspricht der Situation in der freien Feldtheorie, wo z.B. das Wick-Quadrat : $\phi^{2}$ : eines reellen skalaren Feldes dadurch ausgezeichnet wird, daß $\left(\Omega\left|: \phi^{2}:\right| \Omega\right)=0$. Der Raum der "Referenzzustände“ ist in unserem Rahmen allerdings als eine zusätzliche Struktur anzusehen, die durch die Phasenraumeigenschaften der Theorie nicht eindeutig vorgegeben wird.

Symmetrieeigenschaften und „Ordnung“ der Felder Der von uns konstruierte Feldinhalt $\Phi_{\mathrm{FH}}=\bigcup_{\bar{\gamma}} \Phi_{\bar{\gamma}}$ weist durch seinen Aufbau aus endlichdimensionalen Räumen eine „Ordnungsstruktur" nach dem Parameter $\bar{\gamma}$ auf, und das Beispiel der freien Feldtheorie (vgl. Abschnitt 7.4.2) zeigt, daß die Wickprodukte im Normalproduktraum Felder „von höchster Ordnung" sind, während man Felder niedriger Ordnung wie etwa den Einsoperator nicht als Normalprodukt von Feldern bezeichnen würde. Auch im allgemeinen Fall möchte man daher Felder höchster Ordnung in $\mathrm{N}[\Pi]$ auszeichnen.

Dazu nehmen wir an, daß ein $\bar{\gamma}$ existiert mit $\mathrm{N}[\Pi] \cap \Phi_{\bar{\gamma}} \subsetneq \mathrm{N}[\Pi]$, und wählen $\bar{\gamma}$ maximal mit dieser Eigenschaft. Dann kommen Elemente von $\mathrm{N}[\Pi] \cap \Phi_{\bar{\gamma}}$ also nicht als Normalprodukte von $\Pi$ in Betracht. Damit ist aber noch nicht geklärt, wie ein dazu komplementärer Vektorraum $V$ von „möglichen Normalprodukten“ gewählt werden soll, so daß

$$
\mathrm{N}[\Pi]=\left(\mathrm{N}[\Pi] \cap \Phi_{\bar{\gamma}}\right) \oplus V .
$$

Eine Möglichkeit hierzu bieten die Lorentztransformationen: Wir wählen $\bar{\gamma}^{\prime}$, so daß N[ח] $\subset$ $\Phi_{\bar{\gamma}^{\prime}}$. Dann ist $\Phi_{\bar{\gamma}} \subset \Phi_{\bar{\gamma}^{\prime}}$ stabil unter den Darstellern $\alpha(\Lambda)$ der Lorentzgruppe. Da deren 
Darstellungen vollständig reduzibel sind, können wir ein $V_{0} \subset \Phi_{\bar{\gamma}^{\prime}}$ finden, das ebenfalls unter den $\alpha(\Lambda)$ stabil ist, so daß

$$
\Phi_{\bar{\gamma}^{\prime}}=\Phi_{\bar{\gamma}} \oplus V_{0} .
$$

Man wird in (6.2.25) dann $V=V_{0} \cap \mathrm{N}[\Pi]$ betrachten. Allerdings ist weder die Wahl von $V_{0}$ immer eindeutig, noch wird $V$ im allgemeinen eindimensional sein. Trotzdem kann dieses Verfahren weitere Restriktionen für die Auswahl von Normalprodukten liefern.

In konkreten Modellen wird man die Normalprodukte unter Umständen so auswählen wollen, daß sie sich unter einer bestimmten irreduziblen Darstellung der Lorentzgruppe transformieren, etwa einer Vektordarstellung im Fall eines Stroms [.Toh61, Som63]. Ob sich so ein Darstellungsraum in den $\mathrm{N}[\Pi]$ finden läßt (und ob er ggf. eindeutig ist), läßt sich aber ohne weitere Informationen aus der Kovarianzeigenschaft (6.2.22) nicht ablesen. Diese garantiert nur, daß eine einmal getroffene Wahl eines „konkreten“ Normalprodukts für ein Produkt $\Pi$ konsistent auf alle Produkte fortgesetzt werden kann, die aus $\Pi$ durch Symmetrietransformationen hervorgehen.

Feldgleichungen Das Vorhandensein einer Feldgleichung in der Theorie (wir werden dies in Abschnitt 6.4 noch genauer diskutieren) kann unter Umständen ebenfalls zur Auszeichnung eines eindimensionalen Unterraums herangezogen werden. Beispielsweise erwartet man in einer Theorie mit $\phi^{4}$-Kopplung eine Feldgleichung [Zim67], die in unserem Rahmen zunächst ausgedrückt werden kann als

$$
\left(\square+m^{2}\right) \phi \in \mathrm{N}\left[\phi^{3}\right] .
$$

Die linke Seite kann nun zur Definition eines „,verallgemeinerten Wickprodukts“ : $\phi^{3}: \in \mathrm{N}\left[\phi^{3}\right]$ verwendet werden, so daß

$$
\left(\square+m^{2}\right) \phi=\lambda: \phi^{3}:,
$$

wobei die „Normierungskonstante“ $\lambda$ unbestimmt bleibt bzw. frei wählbar ist.

\subsection{Lowenstein's rule}

Wir befassen uns nun damit, inwieweit die Normalproduktbildung mit der Differentiation der Felder verträglich ist. Dies ist im Zusammenhang mit nichtlinearen Feldgleichungen, die wir in Abschnitt 6.4 betrachten werden, von Interesse.

Dazu sei $D \in \mathfrak{D}_{k}$ ein Differentialoperator und $\Pi \in \Phi_{\bar{\gamma}}^{\otimes n+1}$. Da $D$ den Raum $\bar{\Sigma}^{*}$ in sich abbildet, ist $D(\Pi(x))$ für $x \in \mathcal{M}_{\mathrm{R}}^{n+1}$ eine wohldefinierte Linearform. Man erwartet, daß sie sich wieder als raumartiges Produkt von Operatoren schreiben läßt $-D$ sollte hier via Produktregel auf die einzelnen Tensorfaktoren von $\Pi$ wirken.

Um dies genauer zu behandeln, definieren wir zunächst eine Wirkung von $D$ als lineare Abbildung $\Phi_{\bar{\gamma}}^{\otimes n+1} \rightarrow \Phi_{\bar{\gamma}^{\prime}}^{\otimes n+1}$ mit zu $\bar{\gamma}$ geeignetem $\bar{\gamma}^{\prime}$, und zwar wie folgt: Differentialoperatoren $D_{\mu}$ erster Ordnung wirken durch eine „formale Produktregel“

$$
D_{\mu}\left(\phi^{(0)} \otimes \ldots \otimes \phi^{(n)}\right):=\sum_{j=0}^{n} \phi^{(0)} \otimes \ldots \otimes\left(D_{\mu} \phi^{(j)}\right) \otimes \ldots \otimes \phi^{(n)}
$$


Differentialoperatoren höherer Ordnung entsprechend durch Hintereinanderausführung. Wir zeigen dann, daß diese Definition mit der Wirkung von $D$ auf den raumartigen Produkten kompatibel ist:

Lemma 6.4. Sei $D \in \mathfrak{D}_{k}$ ein Differentialoperator und $\Pi \in \Phi_{\bar{\gamma}}^{\otimes n+1}$. Dann gilt

$$
D(\Pi(x))=(D \Pi)(x) .
$$

Beweis. Es reicht wiederum, die „elementaren“ Differentialoperatoren $D_{\mu}$ und Vektoren $\Pi=\phi^{(0)} \otimes \ldots \otimes \phi^{(n)}$ zu betrachten. Wir wählen zunächst approximierende Folgen $A_{r}^{(j)} \in$ $\mathfrak{A}(r)$ nach Satz 3.18, und zwar so, daß auch $\hat{A}_{r}^{(j)}:=A_{r}^{(j)}\left(f_{r}\right)$ die Felder $\phi^{(j)}$ approximieren (zu Details und Bezeichnungsweisen siehe dort). Nach Satz 5.2 gilt dann

$$
\sigma\left(\hat{A}_{r}^{(0)}\left(x_{0}\right) \cdot \ldots \cdot \hat{A}_{r}^{(n)}\left(x_{n}\right)\right) \underset{r \rightarrow 0}{\longrightarrow} \sigma\left(\phi^{(0)}\left(x_{0}\right) \cdot \ldots \cdot \phi^{(n)}\left(x_{n}\right)\right)=\sigma(\Pi(x))
$$

für energiebeschränkte Funktionale $\sigma$. Weil $D_{\mu}=i\left[P_{\mu}, \cdot\right]$ die Energiebeschränkung erhält, mit den Translationen vertauscht und auf $\mathfrak{B}(\mathcal{H})$ der Produktregel genügt, erhalten wir

$$
\sum_{j=0}^{n} \sigma\left(\hat{A}_{r}^{(0)}\left(x_{0}\right) \cdot \ldots \cdot D_{\mu} \hat{A}_{r}^{(j)}\left(x_{j}\right) \cdot \ldots \cdot \hat{A}_{r}^{(n)}\left(x_{n}\right)\right) \underset{r \rightarrow 0}{\longrightarrow} \sigma\left(D_{\mu}(\Pi(x))\right) .
$$

Da die $\hat{A}_{r}^{(j)}$ von der Form $A_{r}^{(j)}\left(f_{r}\right)$ sind, haben wir weiter

$$
D_{\mu} \hat{A}_{r}^{(j) \stackrel{(4.4 .23)}{=}}-A_{r}^{(j)}\left(\partial_{\mu} f_{r}\right) \in \mathfrak{A}(r),
$$

und es gilt die Abschätzung

$$
\left\|R^{l+1}\left(D_{\mu} \hat{A}_{r}^{(j)}-D_{\mu} \phi^{(j)}\right) R^{l+1}\right\|=\left\|R\left[P_{\mu}, R^{l}\left(\hat{A}_{r}^{(j)}-\phi^{(j)}\right) R^{l}\right] R\right\| \leq r \cdot \text { const. }
$$

Satz 5.2 liefert dann

$$
\begin{aligned}
& \sigma\left(\hat{A}_{r}^{(0)}\left(x_{0}\right) \cdot \ldots \cdot D_{\mu} \hat{A}_{r}^{(j)}\left(x_{j}\right) \cdot \ldots \cdot \hat{A}_{r}^{(n)}\left(x_{n}\right)\right) \\
& \underset{r \rightarrow 0}{\longrightarrow} \sigma\left(\phi^{(0)}\left(x_{0}\right) \cdot \ldots \cdot D_{\mu} \phi^{(j)}\left(x_{j}\right) \cdot \ldots \cdot \phi^{(n)}\left(x_{n}\right)\right) .
\end{aligned}
$$

Das ergibt zusammen mit $(6.3 .3)$ die Behauptung des Lemmas.

Wir haben damit die Differentialoperatoren $D$ als lineare Operatoren $D: \Phi_{\bar{\gamma}}^{\otimes n+1} \rightarrow$ $\Phi_{\bar{\gamma}^{\prime}}^{\otimes n+1}$ auf die Räume von Produkten übertragen. Es ist dann naheliegend, nach den Relationen zwischen

$$
D \mathrm{~N}[\Pi] \text { und } \mathrm{N}[D \Pi]
$$

zu fragen - tatsächlich werden wir zeigen, daß die beiden Räume gleich sind. Dazu beweisen wir zunächst folgendes Lemma:

Lemma 6.5. Es sei $V \subset \bar{\Sigma}^{*}$ ein endlichdimensionaler Vektorraum und $D \in \mathfrak{D}_{k}$ ein Differentialoperator. Dann gibt es reguläre Projektionen $p_{V}$ auf $V$ und $p_{D V}$ auf $D V$, so daß

$$
D \circ p_{V}=p_{D V} \circ D \text {. }
$$


Beweis. Wir wählen eine Basis $\left\{\phi_{1}, \ldots, \phi_{J}\right\}$ von $\operatorname{Kern} D \subset V$ und ergänzen sie durch Vektoren $\hat{\phi}_{1}, \ldots, \hat{\phi}_{K}$ zu einer Basis von $V$, so daß dann $\left\{D \hat{\phi}_{1}, \ldots, D \hat{\phi}_{K}\right\}$ eine Basis von $D V$ ist. Nach dem aus (4.A.2) bekannten Verfahren können wir nun Funktionale $\hat{\sigma}_{1}, \ldots, \hat{\sigma}_{K} \in$ $\bar{\Sigma}^{*}$ finden, so daß

$$
\hat{\sigma}_{j}\left(D \hat{\phi}_{k}\right)=\delta_{j k} \quad(j, k=1 \ldots K)
$$

und weiter $\sigma_{1}, \ldots, \sigma_{J} \in \bar{\Sigma}^{*}$, so daß

$$
\sigma_{j}\left(\phi_{k}\right)=\delta_{j k} \quad(j, k=1 \ldots J), \quad \sigma_{j}\left(\hat{\phi}_{k}\right)=0 \quad(j=1 \ldots J, k=1 \ldots K) .
$$

Wir setzen nun

$$
p_{D V}:=\sum_{k=1}^{K} \hat{\sigma}_{k}(\cdot) D \hat{\phi}_{k}, \quad p_{V}:=\sum_{j=1}^{J} \sigma_{j}(\cdot) \phi_{j}+\sum_{k=1}^{K} \hat{\sigma}_{k}(D \cdot) \hat{\phi}_{k}
$$

Diese Abbildungen sind von der Form (4.A.1), wobei man beachtet, daß mit $\sigma_{k}(\cdot)$ auch $\sigma_{k}(D \cdot)$ energiebeschränkt ist. Weiter rechnet man mit Hilfe von (6.3.8) und (6.3.9) sofort nach, daß $p_{V}^{2}=p_{V}, p_{D V}^{2}=p_{D V}$. Die in den Summen auftretenden Funktionale sind nach Definition linear unabhängig. Also ist $p_{V}$ eine reguläre Projektion auf $V, p_{D V}$ entsprechend auf $D V$. Da $\phi_{j} \in \operatorname{Kern} D$, ergibt sich außerdem

$$
D \circ p_{V}=\sum_{k=1}^{K} \hat{\sigma}_{k}(D \cdot) D \hat{\phi}_{k}=p_{D V} \circ D
$$

womit das Lemma bewiesen ist.

Hieraus können wir für die Normalprodukte folgendes schließen: Es sei $\Pi \in \Phi_{\bar{\gamma}}^{\otimes n+1}$ und $V$ raumartig approximierend für $\Pi$. Mit den im obigen Lemma konstruierten Projektionen gilt dann

$$
\begin{aligned}
& \left\|D \Pi(x)-p_{D V} D \Pi(x)\right\|_{E}=\left\|D \Pi(x)-D p_{V} \Pi(x)\right\|_{E} \\
& \quad \leq\|D\|_{E}\left\|\Pi(x)-p_{V} \Pi(x)\right\|_{E} \underset{\mathrm{R}}{\longrightarrow} \quad 0 \quad \forall E>0 ;
\end{aligned}
$$

also ist $D V$ approximierend für $D \Pi$, d.h. es gilt $D V \supset \mathrm{N}[D \Pi]$. Insbesondere ist das für $V=\mathrm{N}[\Pi]$ der Fall, weshalb wir erhalten:

$$
\mathrm{N}[D \Pi] \subset D \mathrm{~N}[\Pi]
$$

Um auch die umgekehrte Inklusion zu zeigen, zerlegen wir $\mathrm{N}[\Pi]$ in eine direkte Summe

$$
\mathrm{N}[\Pi]=V_{0} \oplus V_{1} \oplus V_{2},
$$

wobei

$$
V_{0}=\operatorname{Kern} D \cap \mathrm{N}[\Pi], \quad D V_{1}=\mathrm{N}[D \Pi], \quad D V_{2} \cap \mathrm{N}[D \Pi]=\{0\}
$$


Das ist nach (6.3.13) offenbar möglich. Wir wählen reguläre Projektionen $p_{V}$ auf $V=\mathrm{N}[\Pi]$, $p_{D V}$ auf $D V$ wie in Lemma 6.5. Dabei können wir $p_{V}$ schreiben als

$$
p_{V}=p_{V_{0}}+p_{V_{1}}+p_{V_{2}}, \quad \text { wobei } p_{V_{j}} \text { auf } V_{j} \text { projiziert, }
$$

und zwar so, daß

$$
D p_{V_{j}}=p_{D V_{j}} D \text { für } j=1,2 \text { mit Projektionen } p_{D V_{j}} \text { auf } D V_{j},
$$

wie man dem Beweis des Lemmas entnimmt. Wegen $D p_{V_{0}}=0$ gilt dann

$$
\left\|D \Pi(x)-\left(D p_{V_{1}}+D p_{V_{2}}\right) \Pi(x)\right\|_{E}=\left\|D \Pi(x)-D p_{V} \Pi(x)\right\|_{E} \underset{\mathrm{R}}{\stackrel{(6.3 .12)}{\longrightarrow}} 0 \quad \forall E>0 .
$$

Andererseits ist

$$
\left\|D \Pi(x)-D p_{V_{1}} \Pi(x)\right\|_{E}=\left\|D \Pi(x)-p_{D V_{1}} D \Pi(x)\right\|_{E} \underset{\mathrm{R}}{\longrightarrow} 0 \quad \forall E>0,
$$

da $D V_{1}=\mathrm{N}[D \Pi]$ raumartig approximierend für $D \Pi$ ist, und damit folgt

$$
\left\|D p_{V_{2}} \Pi(x)\right\|_{E} \underset{\mathrm{R}}{\longrightarrow} 0 \quad \forall E>0 .
$$

Da aber $D$ auf dem Raum $V_{2}$ nach Definition invertierbar ist, erhalten wir

$$
\left\|p_{V_{2}} \Pi(x)\right\|_{E} \underset{\mathrm{R}}{\longrightarrow} 0 \quad \forall E>0
$$

Daraus folgt offenbar

$$
\left\|\Pi(x)-\left(p_{V_{0}}+p_{V_{1}}\right) \Pi(x)\right\|_{E} \underset{\mathrm{R}}{\longrightarrow} 0 \quad \forall E>0
$$

d.h. $V_{0} \oplus V_{1}$ ist raumartig approximierend für $\Pi$. Wegen der Minimalität von $V=\mathrm{N}[\Pi]$ (vgl. Satz 6.3) kann das nur für $\operatorname{dim} V_{2}=0$ gelten; daher ist

$$
D \mathrm{~N}[\Pi]=D V_{1}=\mathrm{N}[D \Pi] \text {. }
$$

Wir haben damit bewiesen:

Satz 6.6. Sei $\bar{\gamma}>0, \Pi \in \Phi_{\bar{\gamma}}^{\otimes n+1}$ und $D \in \mathfrak{D}_{k}$ ein Differentialoperator. Dann gilt

$$
\mathrm{N}[D \Pi]=D \mathrm{~N}[\Pi]
$$

Diese Aussage (bzw. ihr störungstheoretisches Analogon) wird in der Literatur als „Lowenstein's rule" bezeichnet [KK.92].

In den Beweis dieser Eigenschaft (von Lemma 6.5 an) geht nicht direkt ein, daß es sich bei $D$ um einen Differentialoperator handelt. Wir haben lediglich verwendet, daß $D$ ein linearer Operator $\bar{\Sigma}^{*} \rightarrow \bar{\Sigma}^{*}$ ist, der den Feldinhalt $\Phi_{\mathrm{FH}}$ in sich überführt und der in gewisser Weise mit den raumartigen Produkten kompatibel ist. Lowenstein's rule gilt also in einem deutlich allgemeineren Kontext. Man kann z.B. die Kovarianzeigenschaft (6.2.22) des Normalproduktraums als Spezialfall von Satz 6.6 auffassen. 


\subsection{Feldgleichungen}

Die Möglichkeit der Differentiation innerhalb des Feldinhalts $\Phi_{\mathrm{FH}}$ (Abschnitte 4.4, 6.3) und die Definition von Normalprodukten (Abschnitt 6.2) erlauben uns, Feldgleichungen zwischen den konstruierten Punktfeldern zu betrachten. Konzeptionell gehen wir dabei aus von einem gegebenen Modell, dessen Punktfeldinhalt wir auf das Vorhandensein von partiellen Differentialgleichungen untersuchen.

Wir entwickeln zunächst einen Formalismus zur Untersuchung linearer Differentialgleichungen, den wir dann weiter unten auf nichtlineare Differentialgleichungen verallgemeinern.

Lineare Feldgleichungen Gegeben sei ein endlichdimensionaler Raum $V \subset \Phi_{\mathrm{FH}}$, den wir daraufhin untersuchen wollen, ob dort lineare Differentialgleichungen erfüllt sind. Ist $D \in \mathfrak{D}_{k}$ ein Differentialoperator höchstens $k$-ter Ordnung, dann wäre eine solche Gleichung etwa gegeben durch

$$
D \phi=0 \quad \text { mit einem } \phi \in V .
$$

Wir formalisieren dies etwas allgemeiner und betrachten dazu folgende Abbildung:

$$
\begin{aligned}
\nabla_{k}: \quad \mathfrak{D}_{k} \times V & \rightarrow \Phi_{\bar{\gamma}^{\prime}}, \\
(D, \phi) & \mapsto D \phi .
\end{aligned}
$$

( $\bar{\gamma}^{\prime}$ ist geeignet zu wählen.) Die Abbildung ist bilinear und läßt sich daher eindeutig zu einer linearen Abbildung

$$
\nabla_{k}^{\otimes}: \quad \mathfrak{D}_{k} \otimes V \rightarrow \Phi_{\bar{\gamma}^{\prime}}
$$

fortsetzen. Der Kern dieser linearen Abbildung kann nun als „Raum der Feldgleichungen“ verstanden werden: Für ein Element $\sum_{j} c_{j} D_{j} \otimes \phi_{j}$ des Tensorprodukts hat man gerade

$$
\sum_{j} c_{j} D_{j} \otimes \phi_{j} \in \operatorname{Kern} \nabla_{k}^{\otimes} \Leftrightarrow \sum_{j} c_{j} D_{j} \phi_{j}=0 .
$$

Auf diese Art können wir unter anderem Gleichungen der folgenden Typs darstellen:

- „Triviale“ Gleichungen wie $\eta^{\mu \nu} D_{\nu} \phi=j^{\mu}$;

- Kontinuitätsgleichungen der Art $D_{\mu} j^{\mu}=0$;

- lineare Feldgleichungen im eigentlichen Sinne, wie etwa die Klein-Gordon-Gleichung $\quad\left(\eta^{\mu \nu} D_{\mu} D_{\nu}+m^{2}\right) \phi=0$.

Hierbei gibt es in unserem Formalismus zumindest a priori keine Möglichkeit, zwischen eigentlichen „Feldgleichungen" und anderen Differentialgleichungen zu unterscheiden, etwa im Sinne des Zeitschichtaxioms. Heuristisch kann man die Lösbarkeit des „klassischen“ Cauchy-Problems als Kriterium heranziehen; eine Konstruktion von Lösungen solcher Gleichungen zwischen Punktfeldern behandeln wir hier allerdings nicht. 
Der „Raum der Feldgleichungen“ Kern $\nabla_{k}^{\otimes}$ trägt trivialerweise eine lineare Struktur. Auf ihm wirken aber auch Symmetrietransformationen: Ist $\alpha(\Lambda)$ Darsteller einer Lorentztransformation, dann operiert $\alpha(\Lambda)$ nicht nur auf $V$, sondern in der in (4.4.20) beschriebenen Weise auch auf $\mathfrak{D}_{k}$. Folglich ist durch

$$
\alpha(\Lambda)(D \otimes \phi):=(\alpha(\Lambda) D) \otimes(\alpha(\Lambda) \phi)
$$

eine Darstellung auf $\mathfrak{D}_{k} \otimes V$ erklärt, wenn wir annehmen, daß $V$ unter $\alpha(\Lambda)$ stabil bleibt (etwa $V=\Phi_{\bar{\gamma}}$ ). Da wir (4.4.21) nun auch schreiben können als

$$
\nabla_{k}^{\otimes}(\alpha(\Lambda)(D \otimes \phi))=\alpha(\Lambda) \nabla_{k}^{\otimes}(D \otimes \phi)
$$

gilt dann

$$
\operatorname{Kern} \nabla_{k}^{\otimes}=\alpha(\Lambda) \operatorname{Kern} \nabla_{k}^{\otimes}
$$

Wir erhalten durch unsere Konstruktion also stets einen Lorentz-kovarianten Satz von Gleichungen. Entsprechendes kann man für andere Symmetrietransformationen etablieren, wenn sie in geeigneter Weise auf die Differentialoperatoren wirken. (Insbesondere gilt das für innere Symmetrien, die mit den Translationen vertauschen.)

Nichtlineare Feldgleichungen Mit Hilfe des Normalprodukts kann man die oben behandelten linearen Feldgleichungen zu nichtlinearen verallgemeinern. Wir gehen dazu wieder aus von einem endlichdimensionalen Teilraum $V \subset \Phi_{\mathrm{FH}}$. Grob gesagt bilden wir nichtlineare Gleichungen für Elemente von $V$, indem wir zunächst Normalprodukte dieser Elemente berechnen und zwischen diesen dann lineare Gleichungen betrachten.

Zunächst geht es also um die Bildung des Raums „aller Normalprodukte“ in $V$. Für $m \in \mathbb{N}$ setzen wir dazu

$$
\mathrm{N}_{m} V=\operatorname{Span}\left\{\mathrm{N}[\Pi] \mid \Pi \in V^{\otimes m}\right\} .
$$

Nach Satz 6.3 folgt aus $V \subset \Phi_{\bar{\gamma}}(\bar{\gamma}$ geeignet $)$ auch $\mathrm{N}_{m} V \subset \Phi_{\bar{\gamma}^{\prime}}$ mit gewissem $\bar{\gamma}^{\prime}$; also ist $\mathrm{N}_{m} V$ endlichdimensional.

Die lokalen Algebren $\mathfrak{A}(r)=\mathfrak{A}(r)^{\prime \prime}$ enthalten alle den Einsoperator; nach der Definition (3.4.1) gilt dann auch stets $\mathbf{1} \in \Phi_{\mathrm{FH}}$. Wir nehmen im folgenden an, daß auch $V$ den Einsoperator enthält, bzw. nehmen in gegebenenfalls hinzu. Für $\Pi \in V^{\otimes m}$ gilt offenbar

$$
\Pi(x)=(\Pi \otimes 1)(x),
$$

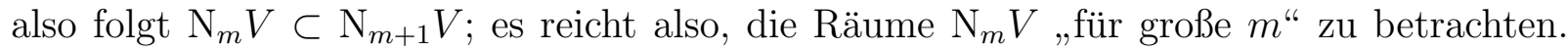
Aus (6.2.17) entnehmen wir

$$
\mathrm{N}[\phi]=\mathbb{C} \phi \forall \phi \in V \quad \Rightarrow \quad \mathrm{N}_{1} V=V \quad \Rightarrow \quad V \subset \mathrm{N}_{m} V \quad \forall m \in \mathbb{N}
$$

der Raum $V$ wird durch das Bilden von Normalprodukten höchstens größer.

Wir schließen auch mehrfache Normalprodukte in unsere Überlegungen mit ein und betrachten für $l \in \mathbb{N}$

$$
\mathrm{N}_{m}^{l} V=\mathrm{N}_{m}\left(\mathrm{~N}_{m}\left(\cdots\left(\mathrm{N}_{m} V\right) \cdots\right) \quad(l \text { Faktoren }) .\right.
$$


Das ist wieder ein endlichdimensionaler Teilraum von $\Phi_{\mathrm{FH}}$. Wir können also den oben entwickelten Formalismus zur Bestimmung linearer Gleichungen auf $\mathrm{N}_{m}^{l} V$ anwenden. Die gesuchten Gleichungen finden wir als Kern der linearen Abbildung

$$
\nabla_{k}^{\otimes}: \mathfrak{D}_{k} \otimes\left(\mathrm{N}_{m}^{l} V\right) \rightarrow \Phi_{\bar{\gamma}^{\prime}} \quad\left(\bar{\gamma}^{\prime} \text { geeignet }\right) .
$$

Auf diese Weise läßt sich z.B. die Feldgleichung

$$
\left(\eta^{\mu \nu} D_{\mu} D_{\nu}+m^{2}\right) \phi=\lambda: \phi^{3}:
$$

darstellen, wobei : $\phi^{3}$ : ein gewisses Element in $\mathrm{N}[\phi \otimes \phi \otimes \phi]$ ist.

Durch Variation von $k, l, m$ können wir den „Feldgleichungsinhalt" der Theorie bestimmen; bezeichnen wir den Kern der Abbildung (6.4.12) als $F_{k l m}$, dann gilt offenbar

$$
F_{k l m} \subset F_{k^{\prime} l^{\prime} m^{\prime}} \quad \text { für } k^{\prime} \geq k, l^{\prime} \geq l, m^{\prime} \geq m \text {. }
$$

Auf $F_{k l m}$ wirkt wie im Fall der linearen Gleichungen eine Darstellung der Lorentzgruppe (vgl. (6.4.5)); ist $V$ unter $\alpha(\Lambda)$ stabil, dann sind es wegen (6.2.22) auch die Normalprodukträume, und wir erhalten

$$
\alpha(\Lambda) F_{k l m}=F_{k l m}
$$

auch die nichtlinearen Feldgleichungen verhalten sich also kovariant unter $\mathfrak{L}$.

Es stellt sich nun die Frage, ob das Verfahren allgemein genug ist, um tatsächlich alle vorhandenen Feldgleichungen zwischen Elementen von $V$ zu beschreiben, ober ob man z.B. durch mehrfach iteriertes Normalprodukt-Bilden und Differenzieren nicht weitere Gleichungen erhält. Formaler lautet die Frage, ob man durch Betrachtung des Raums

$$
\nabla_{k}^{\otimes} \mathrm{N}_{m}^{l} V
$$

und Anwendung des oben beschriebenen Schemas auf diesem Raum statt $V$ neue Feldgleichungen erhält, die bisher nicht sichtbar waren. Tatsächlich ist das nicht der Fall: Als Konsequenz aus Lowenstein's rule werden wir weiter unten zeigen, daß

$$
\nabla_{k}^{\otimes} \mathrm{N}_{m}^{l} V \subset \mathrm{N}_{m}^{l} \mathfrak{D}_{k} V .
$$

Dies bedeutet offenbar, daß

$$
\operatorname{Kern}\left(\nabla _ { k ^ { \prime } } ^ { \otimes } [ \mathrm { N } _ { m ^ { \prime } } ^ { l ^ { \prime } } \nabla _ { k } ^ { \otimes } \mathrm { N } _ { m } ^ { l } V ) \subset \operatorname { K e r n } \left(\nabla_{k^{\prime}}^{\otimes}\left\lceil\mathrm{N}_{\max \left\{m, m^{\prime}\right\}}^{l+l^{\prime}} \mathfrak{D}_{k} V\right),\right.\right.
$$

d.h. man erhält mindestens dieselben Gleichungen, indem man die Parameter $k, l, m$ geeignet ändert und $\mathfrak{D}_{k} V$ statt $V$ betrachtet. Die Verwendung von $\mathfrak{D}_{k} V$ statt $V$ entspricht der Bildung von Gleichungen zwischen Elementen von $V$ mit zusätzlichen linearen Relationen der Form

$$
D v-v^{\prime}=0, \quad v \in V, D \in \mathfrak{D}_{k} .
$$

Wir beweisen nun die Relation (6.4.17). 
Lemma 6.7. Für $k, l, m \in \mathbb{N}$ und endlichdimensionale Teilräume $V \subset \Phi_{\mathrm{FH}}$ gilt

$$
\nabla_{k}^{\otimes} \mathrm{N}_{m}^{l} V \subset \mathrm{N}_{m}^{l} \mathfrak{D}_{k} V
$$

Beweis. Wir zeigen zunächst für beliebige endlichdimensionale Räume $W \subset \Phi_{\mathrm{FH}}$, daß

$$
\mathfrak{D}_{k} \mathrm{~N}_{m} W \subset \mathrm{N}_{m} \mathfrak{D}_{k} W
$$

Das ergibt sich so: Für $D \in \mathfrak{D}_{k}$ hat man

$$
\begin{aligned}
D \mathrm{~N}_{m} W=D \operatorname{Span} & \left\{\mathrm{N}[\Pi] \mid \Pi \in W^{\otimes m}\right\} \\
& =\operatorname{Span}\left\{D \mathrm{~N}[\Pi] \mid \Pi \in W^{\otimes m}\right\}=\operatorname{Span}\left\{\mathrm{N}[D \Pi] \mid \Pi \in W^{\otimes m}\right\}
\end{aligned}
$$

nach Lowenstein's rule (Satz 6.6). Durch Anwendung der Produktregel erhält man Dח $\in$ $\left(\mathfrak{D}_{k} W\right)^{\otimes m}$; daher gilt

$$
D \mathrm{~N}_{m} W \subset \operatorname{Span}\left\{\mathrm{N}[\Pi] \mid \Pi \in\left(\mathfrak{D}_{k} W\right)^{\otimes m}\right\}=\mathrm{N}_{m}\left(\mathfrak{D}_{k} W\right)
$$

womit 6.4 .20 gezeigt ist.

Zum Beweis der Aussage des Lemmas betrachten wir ein allgemeines Element von $\nabla_{k}^{\otimes} \mathrm{N}_{m}^{l} V$; es hat die Form

$$
\sum_{j} c_{j} D_{j} \phi_{j} \quad \operatorname{mit} c_{j} \in \mathbb{C}, D_{j} \in \mathfrak{D}_{k}, \phi_{j} \in \mathrm{N}_{m}^{l} V .
$$

Aus Linearitätsgründen reicht es offenbar zu zeigen, daß

$$
D_{j} \phi_{j} \in \mathrm{N}_{m}^{l} \mathfrak{D}_{k} V
$$

Das folgt aber durch wiederholte Anwendung von (6.4.20):

$$
D_{j} \mathrm{~N}_{m}^{l} V \subset \mathrm{N}_{m} \mathfrak{D}_{k} \mathrm{~N}_{m}^{l-1} V \subset \ldots \subset \mathrm{N}_{m}^{l} \mathfrak{D}_{k} V
$$

wie behauptet. 



\section{Teil III}

\section{Beispiele}





\section{Kapitel 7}

\section{Freie Feldtheorie}

Wir wollen nun die betrachteten Strukturen in konkreten Modellen anwenden und insbesondere das asymptotische Phasenraumkriterium explizit etablieren. Als Beispiel behandeln wir die freie Feldtheorie, also Modelle mit wechselwirkungsfreien Teilchen.

Die freie Feldtheorie wurde aufgrund ihrer vergleichsweise einfachen mathematischen Struktur häufig als Testfall für Phasenraumkriterien herangezogen, siehe etwa [BW86, B.187, BP90]; wir adaptieren teilweise die dort entwickelten Methoden. Auch Haag und Ojima [H()96] verwenden ein solches Modell, um ihre Vermutungen über Zustandskeime plausibel zu machen. Ihre Rechnungen sind jedoch sehr heuristisch; wir präsentieren hier in gewisser Weise eine Präzisierung der Vorstellungen dieser Autoren. Die vorgestellten Ergebnisse wurden in vergleichbarer Form zuerst in [Bos.98] erzielt; die Rechnungen in diesem Kapitel sind größtenteils von dort übernommen.

Nachdem zunächst der Formalismus der freien Feldtheorie in unserem (dem algebraischen) Rahmen kurz wiederholt wird, wenden wir uns der Untersuchung des reellen skalaren freien Feldes zu. Wir etablieren zunächst das asymptotische Phasenraumkriterium. Es zeigt sich, daß das Kriterium im Fall von mindestens $3+1$ Dimensionen erfüllt ist; in niedrigeren Raum-Zeit-Dimensionen ergeben sich hingegen technische Schwierigkeiten (Infrarotdivergenzen).

Wir bestimmen dann die Räume $\Phi_{\bar{\gamma}}$ explizit, indem wir ihre Dimension nach unten abschätzen. Auf diese Weise läßt sich der Fredenhagen-Hertel-Feldinhalt der Theorie vollständig berechnen. Er stimmt mit den bekannten Feldgrößen der Wightman-Theorie (Quantenfeld, Ableitungen, Wick-Produkte) überein, was in einem gesonderten Abschnitt exemplarisch gezeigt wird.

Weiter erläutern wir anhand eines einfachen Beispiels die explizite Berechnung der raumartigen Operatorproduktentwicklung und des Normalproduktraums.

Die Analyse wird nur für den Fall eines reellen skalaren Feldes vollständig durchgeführt. Allerdings lassen sich die Ergebnisse auf gewisse Eigenschaften der Theorie im Einteilchenraum zurückführen, so daß eine Erweiterung auf allgemeinere freie Modelle einfach möglich ist - siehe dazu auch die Diskussion in Kapitel 8 . 


\subsection{Modelle freier Felder}

Wir geben zunächst einen kurzen Überblick über die Formulierung der freien Feldtheorie, genauer der Theorie freier Bosonen, im algebraischen Rahmen. Wir nennen dabei nur die Definitionen und Resultate; eine ausführlichere Darstellung der Konstruktion findet man in [BW.92, Kapitel 8.3].

\subsubsection{Einteilchenraum}

Die Konstruktion beginnt mit dem Einteilchenraum $\mathcal{K}$, einem separablen Hilbertraum mit Skalarprodukt $\langle\cdot \mid \cdot\rangle$, dessen Elemente man als Wellenfunktionen eines Teilchens (,im Impulsraum") deutet. Auf $\mathcal{K}$ hat man eine unitäre Darstellung $U_{\mathcal{K}}(x, \Lambda)$ der Poincaré-Gruppe $\mathfrak{P}$, welche die Spektrumsbedingung erfüllt. Der selbstadjungierte Generator $\omega$ der Zeittranslation kann als Energieoperator interpretiert werden; wir bezeichnen seinen Spektralprojektor auf das Intervall $[0, E]$ mit $Q(E)$.

Weiterhin ist auf $\mathcal{K}$ eine antiunitäre Involution $J$ gegeben, d.h. ein antilinearer Operator mit $J^{2}=\mathbf{1},\langle J f \mid J g\rangle=\langle g \mid f\rangle$. Wir nehmen an, daß $J$ mit $\omega$ kommutiert. Jedes $f \in \mathcal{K}$ besitzt eine eindeutige Zerlegung in $J$-invariante Funktionen der Form

$$
f=f^{+}+i f^{-} \quad, \quad J f^{ \pm}=f^{ \pm}
$$

dabei ist explizit

$$
f^{+}=\frac{1}{2}(1+J) f, \quad f^{-}=\frac{1}{2 i}(1-J) f .
$$

Speziell betrachten wir im folgenden die Theorie eines reellen skalaren freien Teilchens der Masse $m \geq 0$ in $s$ räumlichen Dimensionen. Hier ist der Einteilchenraum gegeben als

$$
\mathcal{K}=L^{2}\left(\mathbb{R}^{s}, d^{s} p\right)
$$

mit dem üblichen Skalarprodukt. Die Zeittranslation wird generiert durch

$$
\omega=\sqrt{\vec{p}^{2}+m^{2}} \quad \text { (als Multiplikationsoperator); }
$$

die Generatoren für räumliche Translationen sind die Multiplikationsoperatoren mit den Koordinaten $p_{j}$. Für die Definition der Darsteller von Lorentztransformationen sei auf [SW64, sect. 1-4.] verwiesen; wir werden sie nicht explizit benötigen.

Vom „Impulsraum“ $L^{2}\left(\mathbb{R}^{s}, d^{s} p\right)$ kann man durch Fouriertransformation zum „Ortsraum" $L^{2}\left(\mathbb{R}^{s}, d^{s} x\right)$ übergehen (zu Vorzeichenkonventionen siehe Seite 201); die Transformation ist unitär, so daß man jedes $f \in \mathcal{K}$ und jeden linearen Operator auf $\mathcal{K}$ wahlweise in einem der beiden Räume betrachten kann. Um die Notation nicht zu überfrachten, werden die beiden Darstellungen im folgenden nicht streng unterschieden, sondern wir deuten lediglich über das Funktionsargument $\vec{x}$ bzw. $\vec{p}$ an, ob wir uns im Orts- oder Impulsraum befinden.

Die Involution $J$ kann nun durch komplexe Konjugation im Ortsraum definiert werden:

$$
(J f)(\vec{x})=\overline{f(\vec{x})} \quad \Leftrightarrow \quad(J f)(\vec{p})=\overline{f(-\vec{p})} .
$$

$J$ hat also die Bedeutung, die Aufspaltung der Wellenfunktionen in Real- und Imaginärteil zu beschreiben respektive (im allgemeinen Fall) diesen Begriff auf beliebige Einteilchenräume zu erweitern. 


\subsubsection{Fockraum}

Die Beschreibung von Mehrteilchenzuständen geschieht mit Hilfe symmetrisierter Tensorprodukte von $\mathcal{K}$, der sogenannten $n$-Teilchen-Räume

$$
\mathcal{H}_{n}:=\operatorname{Symm} \otimes^{n} \mathcal{K} \quad(n \in \mathbb{N}) .
$$

Außerdem setzen wir $\mathcal{H}_{0}:=\mathbb{C} \cdot \Omega$ als eindimensionalen „0-Teilchen-Raum“; physikalisch repräsentiert $\Omega$ das Vakuum. Aus den $\mathcal{H}_{n}$ bildet man nun den Fockraum $\mathcal{H}$ :

$$
\mathcal{H}:=\bigoplus_{n=0}^{\infty} \mathcal{H}_{n} \quad \text { mit Skalarprodukt }(\cdot \mid \cdot)
$$

Mehr von technischer Bedeutung ist der Raum der Vektoren endlicher Teilchenzahl, der aus endlichen Linearkombinationen von Produktvektoren besteht:

$$
\mathcal{H}^{0}:=\operatorname{Span}\left\{\bigoplus_{n=0}^{N} \operatorname{Symm}\left(f_{n 1} \otimes \cdots \otimes f_{n n}\right) \mid N \in \mathbb{N}_{0}, f_{i j} \in \mathcal{K}\right\}
$$

Er ist dicht in $\mathcal{H}$.

Die Darstellung $U_{\mathcal{K}}(x, \Lambda)$ von $\mathfrak{P}$ auf $\mathcal{K}$ bestimmt durch „zweite Quantisierung“ eine Darstellung $U(x, \Lambda)$ auf $\mathcal{H}$. Die Generatoren der Translationen schreiben wir wie zuvor als $P_{\mu}(\mu=0 \ldots s)$; speziell für die Zeittranslation ist das der Hamiltonoperator $H=P_{0}$. Seine Spektralprojektoren auf $[0, E]$ notieren wir wie gehabt als $P(E)$.

$\mathrm{Zu} f \in \mathcal{K}$ hat man die üblichen Erzeugungs- und Vernichtungsoperatoren $a^{*}(f)$ und $a(f)$, die linear bzw. antilinear in $f$ sind und kanonische Vertauschungsrelationen erfüllen:

$$
\left[a(f), a^{*}(g)\right]=\langle f \mid g\rangle \mathbf{1} ; \quad\left[a^{*}(f), a^{*}(g)\right]=0=[a(f), a(g)] \quad(f, g \in \mathcal{K}) .
$$

Sie sind unbeschränkt, aber zumindest auf $\mathcal{H}^{0}$ definiert. Durch Anwendung der Erzeugungsoperatoren $a^{*}(\cdot)$ auf $\Omega$ kann man ganz $\mathcal{H}^{0}$ (und nach Abschluß auch $\mathcal{H}$ ) erhalten, und zwar mit Hilfe folgender Relation:

$$
a^{*}\left(f_{1}\right) \ldots a^{*}\left(f_{n}\right) \Omega=\sqrt{n !} \operatorname{Symm}\left(f_{1} \otimes \cdots \otimes f_{n}\right) ; \quad f_{1}, \ldots, f_{n} \in \mathcal{K} .
$$

Der offenbar symmetrische Operator $a(f)+a^{*}(f)$ ist nach geeigneter Erweiterung des Definitionsbereiches selbstadjungiert [RS75, Theorem X.41]; man kann daher die unitären Operatoren

$$
W(f)=e^{i\left(a(f)+a^{*}(f)\right)}
$$

betrachten. Sie werden als Weyl-Operatoren bezeichnet; wir listen hier einige ihrer Eigenschaften auf:

$$
\begin{gathered}
W(f) W(g)=W(f+g) e^{i \operatorname{Im}<g \mid f>} \\
W(f)=e^{i a^{*}(f)} e^{i a(f)} e^{-\frac{1}{2}\|f\|^{2}} \\
{[a(g), W(f)]=i\langle g \mid f\rangle W(f), \quad\left[a^{*}(g), W(f)\right]=-i\langle f \mid g\rangle W(f) ;} \\
(\Omega \mid W(f) \Omega)=e^{-\frac{1}{2}\|f\|^{2}} .
\end{gathered}
$$




\subsubsection{Lokale Algebren}

Die Definition der lokalen Algebren $\mathfrak{A}(\mathcal{O})$ erfolgt so: Zu jeder offenen Menge $\mathcal{O} \subset \mathcal{M}$ sind zwei abgeschlossene Unterräume $\mathcal{L}^{ \pm}(\mathcal{O}) \subset \mathcal{K}$ gegeben. Sie sind unter $J$ invariant, also $J \mathcal{L}^{ \pm}(\mathcal{O}) \subset \mathcal{L}^{ \pm}(\mathcal{O})$; bezeichnet $P_{\mathcal{L}}^{ \pm}(\mathcal{O})$ den Projektor auf $\mathcal{L}^{ \pm}(\mathcal{O})$, dann impliziert das $\left[J, P_{\mathcal{L}}^{ \pm}(\mathcal{O})\right]=0$. Die Räume $\mathcal{L}^{ \pm}(\mathcal{O})$ hängen mit den Anfangswerten des Cauchyproblems für die zugrundeliegende Feldgleichung zusammen (näheres siehe Abschnitt 7.4). Man betrachtet jetzt folgenden reell-linearen Unterraum von $\mathcal{K}$ :

$$
\mathcal{L}(\mathcal{O})=(1+J) \mathcal{L}^{+}(\mathcal{O})+(1-J) \mathcal{L}^{-}(\mathcal{O}) .
$$

Für $f \in \mathcal{L}(\mathcal{O})$ ist in der Zerlegung (7.1.1) dann $f^{ \pm} \in \mathcal{L}^{ \pm}(\mathcal{O})$.

Die lokale Algebra für $\mathcal{O}$ wird nun von allen zu $\mathcal{L}(\mathcal{O})$ gehörenden Weyloperatoren erzeugt, enthält also deren Linearkombinationen und schwache Limespunkte:

$$
\mathfrak{A}(\mathcal{O}):=\{W(f) \mid f \in \mathcal{L}(\mathcal{O})\}^{\prime \prime} .
$$

Erfüllen die $\mathcal{L}(\mathcal{O})$ Isotonie-, Kovarianz- und Lokalitätsbedingungen, wobei die Lokalität formuliert wird durch

$$
\left\langle f_{1} \mid f_{2}\right\rangle=\left\langle f_{2} \mid f_{1}\right\rangle \text { für raumartig getrennte } \mathcal{O}_{1}, \mathcal{O}_{2} \text { und } f_{i} \in \mathcal{L}\left(\mathcal{O}_{i}\right),
$$

dann bilden die $\mathfrak{A}(\mathcal{O})$ ein lokales Netz, das die Axiome aus Abschnitt 1.3.1 erfüllt. Die Darstellung der Poincaré-Gruppe $\mathfrak{P}$ ist dabei per Definition unitär implementiert:

$$
\alpha(x, \Lambda):=U(x, \Lambda) \cdot U(x, \Lambda)^{*} .
$$

Für das reelle skalare Feld werden die Räume $\mathcal{L}^{ \pm}$wie folgt definiert: Für offene StandardDoppelkegel $\mathcal{O}_{r}$ vom Radius $r$ mit Mittelpunkt 0 (wie in (1.3.7) definiert) setzt man

$$
\mathcal{L}^{ \pm}\left(\mathcal{O}_{r}\right):=\overline{\omega^{\mp \frac{1}{2}} \mathcal{D}_{\mathbb{C}}(r)} .
$$

Dabei bezeichnet $\mathcal{D}_{\mathbb{C}}(r)$ die Menge der komplexwertigen Schwartzfunktionen auf $\mathbb{R}^{s}$, deren Träger im Ortsraum innerhalb der Kugel $|\vec{x}|<r$ liegt. Damit sind die $\mathcal{L}(\mathcal{O})$ für eine Nullumgebungsbasis erklärt; durch Anwendung der Translationen $U_{\mathcal{K}}(x)$ erhält man sie für Umgebungsbasen beliebiger $x$, für allgemeines $\mathcal{O}$ dann per Additivität. Für unsere Analyse genügt es, Doppelkegel $\mathcal{O}_{r}$ zu betrachten; wir schreiben daher auch kurz $\mathcal{L}^{ \pm}\left(\mathcal{O}_{r}\right)=\mathcal{L}^{ \pm}(r)$ und $P_{\mathcal{L}}^{ \pm}\left(\mathcal{O}_{r}\right)=P_{\mathcal{L}}^{ \pm}(r)$.

\subsection{Nachweis des Phasenraumkriteriums}

Unser Ziel ist es, das in Definition 2.1 formulierte asymptotische Phasenraumkriterium mit polynomialen Energieschranken im beschriebenen Modell explizit nachzuprüfen. Wir haben also zu jedem $\bar{\gamma}>0$ eine Abbildung $\psi \in \bar{\Psi}_{0}^{P}$ von asymptotisch endlichem Rang anzugeben, so daß

$$
\gamma(\Xi-\psi) \geq \bar{\gamma}
$$


Wir betrachten dazu zunächst $\Xi_{E, r}$ bei festem $E$ und $r$ und leiten eine Entwicklung dieser Abbildung in Rang-1-Terme her, also eine Reihendarstellung

$$
\Xi_{E, r}=\sum_{j} \phi_{j} \sigma_{j}
$$

Das Verhalten im Limes kleiner Wirkungen $(E r \rightarrow 0)$ wird dann später diskutiert.

Im Zusammenhang mit der von ihnen untersuchten Nuklearitätsbedingung hatten schon Buchholz und Porrmann [BP90] eine Entwicklung von $\Xi_{E, r}$ nach Rang-1-Operatoren angegeben. I In dieser Arbeit lag das Augenmerk aber hauptsächlich auf einer Abschätzung der $p$-Normen von $\Xi_{E, r}$; die einzelnen Terme der Reihenentwicklung waren ohne Belang. Buchholz [Buc95] gelang es später, die dort verwendeten Konzepte so zu erweitern, daß auch die Normen der einzelnen Approximationsterme $\phi_{j} \sigma_{j}$ und ihr Verhalten mit $E$ und $r$ kontrolliert werden konnten.

Für unsere Zwecke weist der Ansatz dieser Autoren aber einen entscheidenden Mangel auf: Die von ihnen berechneten $\sigma_{j}$ und $\phi_{j}$ hängen in subtiler und kaum zu kontrollierender Weise von $E$ und $r$ ab. Wir möchten jedoch letztlich eine Entwicklung der Form (7.2.2) mit $E$ - und $r$-unabhängigen Termen erhalten, d.h. eine Reihenentwicklung im Sinne bilinearer Abbildungen auf $\bar{\Sigma} \times \overline{\mathfrak{A}}$ - diese Form hatte sich in Kapitel 2 als essentiell für die Konstruktion von Punktfeldern erwiesen. Das Verfahren aus [BP90] muß daher in unserem Sinne modifiziert werden.

Gleichzeitig beseitigen wir einen weiteren Nachteil des genannten Konzepts: Die Anzahl der Approximationsterme (für Approximation bis zu einer gegebenen Genauigkeit) ist in [BP90] nicht minimal gewählt - dies ist dort auch nicht relevant, führte aber zu einer Diskrepanz zwischen den von Buchholz [Buc.95] einerseits und Haag/Ojima [H( .96] $^{-}$ andererseits berechneten „Dimensionen der Halme“. Die hier hergeleitete Entwicklung besteht hingegen im beschriebenen Sinn aus minimal vielen Termen, d.h. die lineare Hülle der berechneten $\phi_{j}$ stimmt tatsächlich mit dem Feldinhalt überein. Wir werden dies in Abschnitt 7.3 noch präziser betrachten.

\subsubsection{Vorgehensweise}

Um das Phasenraumkriterium aus Definition 2.1 zu etablieren, haben wir die bilineare Abbildung $\Xi$ durch eine Abbildung $\psi$ von asymptotisch endlichem Rang so zu approximieren, daß

$$
\lim _{w \rightarrow 0} w^{-\bar{\gamma}+\epsilon} \sup _{E r \leq w}\|\Xi-\psi\|_{E, r}=0 \quad \forall \epsilon>0 .
$$

Wir werden uns dazu, wie erwähnt, zunächst auf festes $E$ und $r$ beschränken und die Abbildung $\Xi_{E, r}$ approximieren; genauer erweist es sich als günstig, die Rechtsadjungierte zu betrachten, die wir hier in der Form

$$
\begin{aligned}
\Xi_{\mathrm{R} E, r}: & \mathfrak{A}(r) \rightarrow \Sigma(E)^{*}=P(E) \mathfrak{B}(\mathcal{H}) P(E), \\
& A \mapsto P(E) A P(E)
\end{aligned}
$$

\footnotetext{
${ }^{1}$ In $[\mathrm{BP90}]$ wurde allerdings eine Energiedämpfung mit $e^{-\beta H}$ statt $P(E)$ verwendet.
} 
schreiben. Wir werden für diese Abbildung zwei Reihenentwicklungen nach Rang-1Operatoren angeben, und zwar in der Form

$$
\Xi_{\mathrm{R} E, r}(\cdot)=\sum \sigma_{j}(\cdot) \phi_{j} ; \quad \sigma_{j} \in \Sigma, \phi_{j} \in P(E) \mathfrak{B}(\mathcal{H}) P(E) .
$$

Eine davon besteht aus skalenunabhängigen Termen und läßt sich später zu einer Entwicklung von $\Xi$ fortsetzen, die andere benötigt man nur aus technischen Gründen wegen ihrer guten Konvergenzeigenschaften.

Beide Entwicklungen werden zunächst durch explizite Zerlegung von $\Xi_{\mathrm{R} E, r}(W(f))$ mit lokalisiertem $f$ hergeleitet (Abschnitt 7.2.4); diese Aufgabe läßt sich auf Annahmen über die Eigenschaften der Theorie im Einteilchenraum zurückführen (Abschnitt 7.2.2 und 7.2.3), die wir im Fall des reellen skalaren Feldes explizit nachweisen. Dann diskutieren wir, inwiefern sich die berechnete Zerlegung linear und stetig auf ganz $\mathfrak{A}(r)$ fortsetzen läßt (Abschnitt 7.2.5).

Zuerst aber befassen wir uns mit dem Problem im Einteilchenraum. Im folgenden bezeichne $f^{ \pm}$eine Funktion aus $\mathcal{L}^{ \pm}(r)$, und es sei $k \in Q(E) \mathcal{K}$. Wir interessieren uns für Reihenentwicklungen des Skalarprodukts $\left\langle f^{ \pm} \mid k\right\rangle$ und werden, wie angekündigt, zwei verschiedene solche angeben.

\subsubsection{Entwicklung nach skalenunabhängigen Funktionen}

Zuerst leiten wir eine Reihendarstellung für das besagte Skalarprodukt her, indem wir Projektoren auf explizit bekannte Funktionen im Einteilchenraum „einschieben“.

Dazu fixieren wir eine reellwertige Testfunktion $\chi(x)$ auf $\mathbb{R}$ mit $\chi(x)=1$ für $|x| \leq 1$, $\chi(x)=0$ für $|x| \geq 2$; weiter setzen wir

$$
\chi_{r}(\vec{x}):=\chi\left(\frac{|\vec{x}|}{r}\right), \quad \text { also } \chi \in \mathcal{S}\left(\mathbb{R}^{s}\right) ; \chi_{r}(\vec{x})=1 \text { für }|\vec{x}| \leq r .
$$

Außerdem bezeichnen wir mit $\chi_{E}(\vec{p})$ die charakteristische Funktion

$$
\chi_{E}(\vec{p}):= \begin{cases}1 & \text { für } \omega(\vec{p}) \leq E \\ 0 & \text { sonst. }\end{cases}
$$

Sei zunächst $k \in \mathcal{D}\left(\omega^{-\frac{1}{2}}\right)$ und außerdem glatt. Weiterhin sei $f^{ \pm} \in \omega^{\mp \frac{1}{2}} \mathcal{D}_{\mathbb{C}}(r)$. (Damit werden sowohl $f$ als auch $k$ aus dichten Mengen der interessierenden Räume gewählt.) Wir können dann schreiben:

$$
\left\langle f^{ \pm} \mid k\right\rangle=\left\langle\omega^{ \pm \frac{1}{2}} f^{ \pm} \mid \omega^{\mp \frac{1}{2}} k\right\rangle=\int_{|\vec{x}| \leq r} \overline{\left(\omega^{ \pm \frac{1}{2}} f^{ \pm}\right)(\vec{x})} \cdot \widetilde{\omega^{\mp \frac{1}{2}} k}(\vec{x}) d^{s} x
$$

( $\omega^{\mp \frac{1}{2}} k$ hat im Impulsraum kompakten Träger, die Fouriertransformierte ist also holomorph.)

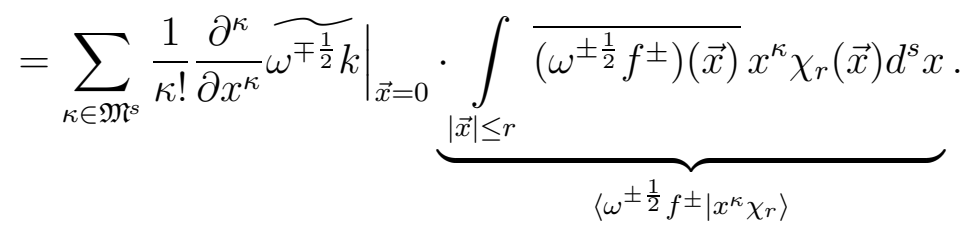


$\kappa$ läuft über alle $s$-stelligen Multiindizes - siehe dazu Anhang 7.2.A. Die auftretende Ableitung läßt sich auch als Skalarprodukt ausdrücken:

$$
\begin{aligned}
\left.\frac{\partial^{\kappa}}{\partial x^{\kappa}} \widetilde{\omega^{\mp \frac{1}{2}} k}\right|_{\vec{x}=0}=\left.(2 \pi)^{-\frac{s}{2}} \frac{\partial^{\kappa}}{\partial x^{\kappa}} \int_{\omega \leq E} \omega^{\mp \frac{1}{2}} k(\vec{p}) e^{i \vec{p} \vec{x}} d^{s} p\right|_{\vec{x}=0} \\
=(2 \pi)^{-\frac{s}{2}} \int_{\omega \leq E} \omega^{\mp \frac{1}{2}} k(\vec{p})(i p)^{\kappa} d^{s} p=\left\langle\frac{i^{-|\kappa|}}{(2 \pi)^{s / 2}} p^{\kappa} \chi_{E} \mid \omega^{\mp \frac{1}{2}} k\right\rangle .
\end{aligned}
$$

Man sieht nun, daß sowohl $x^{\kappa} \chi_{r}$ wie auch $p^{\kappa} \chi_{E}$ im Definitionsbereich von $\omega^{ \pm \frac{1}{2}}$ liegen, falls $s \geq 2$ oder $m>0$ : Für $p^{\kappa} \chi_{E}$ ist das unmittelbar klar, und da $x^{\kappa} \chi_{r}$ eine Testfunktion ist, ist ihre Fouriertransformierte ebenfalls glatt und fällt rasch ab. Damit erhält man insgesamt:2

$$
\left\langle f^{ \pm} \mid k\right\rangle=\sum_{\kappa}\langle f^{ \pm} \mid \underbrace{\frac{\sqrt{2}}{\kappa !} \omega^{ \pm \frac{1}{2}} x^{\kappa} \chi_{r}}_{=: h_{\kappa}^{ \pm}}\rangle\langle\underbrace{\frac{i^{-|\kappa|}}{\sqrt{2}(2 \pi)^{s / 2}} \omega^{\mp \frac{1}{2}} p^{\kappa} \chi_{E}}_{=: g_{\kappa}^{ \pm}} \mid k\rangle=\sum_{\kappa}\left\langle f^{ \pm} \mid h_{\kappa}^{ \pm}\right\rangle\left\langle g_{\kappa}^{ \pm} \mid k\right\rangle .
$$

Die Funktionen $g_{\kappa}^{ \pm}$und $h_{\kappa}^{ \pm}$sind von $E$ bzw. $r$ abhängig definiert (im Widerspruch zum Titel des Abschnitts); sind aber $E^{\prime}, E^{\prime \prime} \geq E$, dann gilt

$$
Q(E) g_{\kappa}^{ \pm\left(E^{\prime}\right)}=Q(E) g_{\kappa}^{ \pm\left(E^{\prime \prime}\right)}
$$

in diesem Sinne können wir sagen, daß die Definition der $g_{\kappa}^{ \pm}$mit den $Q(E)$ verträglich ist, oder wir können in Ausdrücken der Form $Q(E) g_{\kappa}^{ \pm}$die $g_{\kappa}^{ \pm}$als $E$-unabhängige Größen betrachten. Analoges gilt für die $h_{\kappa}^{ \pm}$hinsichtlich der $P_{\mathcal{L}}^{ \pm}(r)$.

Wir werden nun noch die Normen der Vektoren $g_{\kappa}^{ \pm}$und $h_{\kappa}^{ \pm}$, insbesondere deren Verhalten hinsichtlich $E$ und $r$, abschätzen. Allgemein hat man für $\beta>-1$ (und $s \geq 2$ ):

$$
\begin{aligned}
\left\|\omega^{\beta} p^{\kappa} \chi_{E}\right\|^{2}=\int_{\omega \leq E} \omega^{2 \beta} p^{2 \kappa} d^{s} p \leq \int_{|\vec{p}| \leq E} p^{2 \kappa}\left(\vec{p}^{2}+m^{2}\right)^{\beta} d^{s} p \\
\stackrel{\text { (Skalierung) }}{=} E^{s+2 \beta+2|\kappa|} \int_{|\vec{p}| \leq 1} \underbrace{p^{2 \kappa}}_{\leq 1}\left(\vec{p}^{2}+\left(\frac{m}{E}\right)^{2}\right)^{\beta} d^{s} p \\
\leq E^{s+2 \beta+2|\kappa|} \cdot \text { const. etwa für } E>m .
\end{aligned}
$$

Die Konstante hängt zwar von $\beta$, nicht aber von $\kappa$ ab. Mit der Definition

$$
\vartheta^{ \pm}(\kappa):=\frac{s \mp 1}{2}+|\kappa|
$$

erhalten wir also

$$
\left\|g_{\kappa}^{ \pm}\right\| \leq E^{\vartheta^{ \pm}(\kappa)} \cdot c_{g} \quad \text { sowie } \quad\left\|\omega^{-\frac{1}{2}} g_{\kappa}^{ \pm}\right\| \leq E^{\vartheta^{ \pm}(\kappa)-\frac{1}{2}} \cdot c_{g}
$$

2 Die in (7.2.11) scheinbar willkürlich eingeschobenen Faktoren $\sqrt{2}$ dienen zur Normierung der in Abschnitt 7.4 .2 rekonstruierten Punktfelder. 
mit einer Konstanten $c_{g}$.

Die Normen der Funktionen $h_{\kappa}^{ \pm}$lassen sich so abschätzen:

$$
\begin{aligned}
& \left\|\omega^{ \pm \frac{1}{2}} x^{\kappa} \chi_{r}\right\|^{2}=\int d^{s} p{\sqrt{\vec{p}^{2}+m^{2}}}^{ \pm 1}(2 \pi)^{-s}\left|\int x^{\kappa} \chi_{r}(\vec{x}) e^{-i \vec{p} \vec{x}} d^{s} x\right|^{2} \\
& \stackrel{\text { (Skalierung) }^{=}}{=}(2 r)^{s \mp 1+2|\kappa|}(2 \pi)^{-s} \int d^{s} p{\sqrt{\vec{p}^{2}+(2 m r)^{2}}}^{ \pm 1}|\int x^{\kappa} \underbrace{\chi(2|\vec{x}|)}_{\chi_{\frac{1}{2}}(\vec{x})} e^{-i \vec{p} \vec{x}} d^{s} x|^{2} \\
& =(2 r)^{s \mp 1+2|\kappa|}\left\langle x^{\kappa} \chi_{\frac{1}{2}}\left|{\sqrt{\vec{p}^{2}+(2 m r)^{2}}}^{ \pm 1}\right| x^{\kappa} \chi_{\frac{1}{2}}\right\rangle .
\end{aligned}
$$

Im zuletzt stehenden Erwartungswert ist zwar das Verhalten mit $r$ sehr einfach, das mit $\kappa$ jedoch weitaus schwieriger zu kontrollieren. Wir betrachten zunächst den Fall „-“. Hier gilt

$$
{\sqrt{\vec{p}^{2}+(2 m r)^{2}}}^{-1} \leq \frac{1}{|\vec{p}|} .
$$

Den genannten Erwartungswert berechnen wir nun durch Aufspalten des Integrationsbereiches in $|\vec{p}| \leq 1$ und $|\vec{p}| \geq 1$ :

$$
\begin{aligned}
& \left\langle x^{\kappa} \chi_{\frac{1}{2}}\left|\frac{1}{|\vec{p}|}\right| x^{\kappa} \chi_{\frac{1}{2}}\right\rangle \\
& =(2 \pi)^{-s} \int_{|\vec{p}| \leq 1} d^{s} p \frac{1}{|\vec{p}|}\left|\int x^{\kappa} \chi_{\frac{1}{2}}(\vec{x}) e^{-i \vec{p} \vec{x}} d^{s} x\right|^{2}+\int_{|\vec{p}| \geq 1} d^{s} p \underbrace{\frac{1}{|\vec{p}|}}_{\leq 1}\left|\widetilde{x^{\kappa} \chi_{\frac{1}{2}}}(\vec{p})\right|^{2} \\
& \leq(2 \pi)^{-s} \int_{|\vec{p}| \leq 1} d^{s} p \frac{1}{|\vec{p}|}\left(\int_{|\vec{x}| \leq 1}\left|x^{\kappa}\right|\left|\chi_{\frac{1}{2}}(\vec{x})\right| d^{s} x\right)^{2}+\left\|x^{\kappa} \chi_{\frac{1}{2}}\right\|^{2} \leq \text { const. }
\end{aligned}
$$

Hier wurde $s \geq 2$ verwendet. Im Fall „+" hat man für $2 m r \leq 1$ :

$$
\sqrt{\vec{p}^{2}+(2 m r)^{2}} \leq \vec{p}^{2}+(2 m r)^{2}+1 \leq \vec{p}^{2}+2
$$

und damit

$$
\begin{aligned}
& \left\langle x^{\kappa} \chi_{\frac{1}{2}}\left|\sqrt{\vec{p}^{2}+(2 m r)^{2}}\right| x^{\kappa} \chi_{\frac{1}{2}}\right\rangle \\
& \leq\left\langle x^{\kappa} \chi_{\frac{1}{2}}|-\Delta| x^{\kappa} \chi_{\frac{1}{2}}\right\rangle+2\left\|x^{\kappa} \chi_{\frac{1}{2}}\right\|^{2} \leq \int_{|\vec{x}| \leq 1}\left(\operatorname{grad} x^{\kappa} \chi_{\frac{1}{2}}\right)^{2} d^{s} x+\text { const. } \\
& \leq \int_{|\vec{x}| \leq 1} \sum_{i=1}^{s}(\underbrace{2 \chi^{\prime}(2|\vec{x}|) \cdot \frac{x_{i}}{|\vec{x}|} \cdot x^{\kappa}}_{\leq \text {const.' }}+\kappa_{i} \underbrace{x^{\kappa^{\prime}} \chi_{\frac{1}{2}}(\vec{x})}_{\leq \text {const." }})^{2} d^{s} x+\text { const. } \\
& \quad \begin{array}{c}
\text { Stelle um } 1 \text { vermindert. }) \\
\leq \text { const."' } \cdot \sum_{i} \kappa_{i}^{2}+\text { const. gegenüber } \kappa \text { an der } i \text {-ten }
\end{array}
\end{aligned}
$$


Berücksichtigt man nun, daß $\sum \kappa_{i}^{2} \leq$ const. $\cdot \sqrt{\kappa !}$, dann wird mit einer Konstanten $c_{h}$

$$
\left\|h_{\kappa}^{ \pm}\right\| \leq \frac{(2 r)^{\vartheta^{ \pm}(\kappa)}}{\sqrt[4]{\kappa !}} \cdot c_{h}
$$

Wir kommen jetzt noch einmal auf die Reihenentwicklung (7.2.11) zurück. Nach obigen Abschätzungen gilt für $s \geq 2, E r \leq 1$ und $E>2 m$ :

$$
\sum_{\kappa}\left\|h_{\kappa}^{ \pm}\right\|\left\|g_{\kappa}^{ \pm}\right\| \leq \sum_{\kappa} \frac{(2 E r)^{|\kappa|}}{\sqrt[4]{\kappa !}} \cdot(2 E r)^{\frac{s \mp 1}{2}} \cdot \text { const. } \leq \prod_{i=1}^{s} \sum_{\kappa_{i}=0}^{\infty} \frac{(2 E r)^{\kappa_{i}}}{\sqrt[4]{\kappa_{i} !}} \cdot \text { const. } .^{\prime}<\text { const. }{ }^{\prime \prime}
$$

denn die Potenzreihe

$$
F(z)=\sum_{k=0}^{\infty} \frac{z^{k}}{\sqrt[4]{k !}}
$$

konvergiert für alle $z \in \mathbb{C}$ nach dem Quotientenkriterium. Damit kann die Entwicklung (7.2.11) aber auf alle $f^{ \pm} \in \mathcal{L}^{ \pm}(r)$ und alle $k \in Q(E) \mathcal{K}$ ausgedehnt werden (bisher waren die Funktionen nur aus dichten Teilmengen gewählt). Wir können im Sinne von Normkonvergenz behaupten:

$$
Q(E) P_{\mathcal{L}}^{ \pm}(r)=\sum_{\kappa} Q(E)\left|g_{\kappa}^{ \pm}\right\rangle\left\langle h_{\kappa}^{ \pm}\right| P_{\mathcal{L}}^{ \pm}(r)
$$

Tatsächlich benötigen wir für das folgende nur schwache Konvergenz.

Wir numerieren die $g^{ \pm}$und $h^{ \pm}$nun mit natürlichen Zahlen $j$ statt Multiindizes, und zwar derart, daß das aus (7.2.14) resultierende $\vartheta^{ \pm}(j)$ monoton mit $j$ wächst. Für spätere Anwendungen betrachten wir noch einmal die Summe der Normen dieser Vektoren, wobei wir jetzt die Terme zu niedrigem $j$ fortlassen; mit analogen Argumentationen wie in (7.2.22) erhalten wir für $n \in \mathbb{N}$ :

$$
\sum_{j=n}^{\infty}\left\|h_{j}^{ \pm}\right\|\left\|E^{\frac{1}{2}} \omega^{-\frac{1}{2}} g_{j}^{ \pm}\right\| \leq(2 E r)^{\vartheta^{ \pm}(n)} \cdot \text { const }
$$

Daß wir hier die $E^{\frac{1}{2}} \omega^{-\frac{1}{2}} g_{j}^{ \pm}$statt der $g_{j}^{ \pm}$verwenden, macht wegen (7.2.15) keinen Unterschied.

Wir formulieren nun für den allgemeinen Fall als Forderung, was wir für das reelle skalare Feld in $s \geq 2$ Raumdimensionen gezeigt haben:

Eigenschaft 7.1. $Z u r \leq r_{0}, E \geq E_{0}, E r \leq 1$ gibt es Vektoren $g_{j}^{ \pm}, h_{j}^{ \pm} \in \mathcal{K}(j \in \mathbb{N})$, verträglich mit den $Q(E)$ bzw. $P_{\mathcal{L}}^{ \pm}(r)$, so daß

$$
Q(E) \cdot P_{\mathcal{L}}^{ \pm}(r)=\sum_{j=1}^{\infty} Q(E)\left|g_{j}^{ \pm}\right\rangle\left\langle h_{j}^{ \pm}\right| P_{\mathcal{L}}^{ \pm}(r)
$$

\footnotetext{
${ }^{3}$ Für eine genauere Formulierung dieser Eigenschaft siehe $(7.2 .12)$ und die zugehörige Diskussion.
} 
im Sinne schwacher Konvergenz. Die Funktionen $g_{j}^{ \pm}$liegen im Definitionsbereich von $\omega^{-\frac{1}{2}}$; die $h_{j}^{ \pm}$sind invariant unter $J$. Weiter existieren zwei monoton wachsende Funktionen $\vartheta^{ \pm}: \mathbb{N} \rightarrow \mathbb{R}^{+}$mit $\vartheta^{ \pm}(n) \rightarrow \infty(n \rightarrow \infty)$, so daß

$$
\sum_{j=n}^{\infty}\left\|E^{\frac{1}{2}} Q(E) \omega^{-\frac{1}{2}} g_{j}^{ \pm}\right\|\left\|P_{\mathcal{L}}^{ \pm}(r) h_{j}^{ \pm}\right\| \leq(c E r)^{\vartheta^{ \pm}(n)} \cdot \text { const. } \quad \forall n \in \mathbb{N} .
$$

Dabei sind $E_{0}, r_{0}$ und c positive Konstanten.

Aus dieser Eigenschaft folgt offenbar auch im allgemeinen Fall

$$
\left\|E^{\frac{1}{2}} P(E) \omega^{-\frac{1}{2}} g_{j}^{ \pm}\right\| \cdot\left\|P_{\mathcal{L}}^{ \pm}(r) h_{j}^{ \pm}\right\| \leq(c E r)^{\vartheta^{ \pm}(j)} \cdot \text { const. }
$$

- für das reelle skalare Feld war das bereits klar. Insbesondere sind die $\left\|E^{\frac{1}{2}} P(E) \omega^{-\frac{1}{2}} g_{j}^{ \pm}\right\|$ für $E \rightarrow \infty$ polynomial beschränkt.

\subsubsection{Entwicklung nach skalenabhängigen Funktionen}

Die oben beschriebene Entwicklung verwendet explizit bekannte Funktionen $g_{j}^{ \pm}$und $h_{j}^{ \pm}$. Diese sind jedoch nicht orthogonal zueinander; das stellt sich bei der Analyse der $W(f)$ als hinderlich heraus, wenn man Anteile für hohe Teilchenzahlen abschätzen will. Wir benötigen daher eine weitere Entwicklung im Einteilchenraum nach einem Orthonormalsystem; dies lehnt sich an die in [BP90] verwendete Methode an. Wir betrachten dazu folgende Operatoren:

$$
T^{ \pm}(E, r):=\omega^{-\frac{1}{2}} Q(E) P_{\mathcal{L}}^{ \pm}(r) .
$$

Die von ihnen geforderte Eigenschaft formulieren wir gleich allgemein:

Eigenschaft 7.2. Die Operatoren $T^{ \pm}(E, r)=\omega^{-\frac{1}{2}} Q(E) P_{\mathcal{L}}^{ \pm}(r)$ sind in der Spurklasse, und für ihre Spurnormen gilt mit einem $\alpha>0$ :

$$
\left\|T^{ \pm}(E, r)\right\|_{1} \leq E^{-\frac{1}{2}}(E r)^{\alpha} \cdot \text { const. für } r \leq r_{0}, E \geq E_{0}, E r \leq 1 .
$$

Der Nachweis dieser Eigenschaft für das reelle skalare Feld wird in Anhang 7.2.9 geführt; sie läßt sich in $s \geq 3$ Raumdimensionen etablieren.

Wir schreiben nun $\left|T^{ \pm}\right|=\left(T^{ \pm *} T^{ \pm}\right)^{\frac{1}{2}}$ und bezeichnen die kleinste obere Schranke $\left.\right|^{\natural}$ von $\left|T^{+}\right|$und $\left|T^{-}\right|$mit $T$. Dann ist $T$ nach Lemma 7.15 in Anhang 7.2.D ebenfalls in der Spurklasse, und die Spurnorm erfüllt Schranken vom in Eigenschaft 7.2 genannten Typ. Die der Größe nach geordneten Eigenwerte von $T$ seien $t_{j}, j \in \mathbb{N}$, und die zugehörigen Eigenvektoren benennen wir mit $e_{j}$. Die Involution $J$ vertauscht mit $\omega$ und $P_{\mathcal{L}}^{ \pm}$, also auch mit den $T^{ \pm}$und ihren Adjungierten, damit auch mit $\left|T^{ \pm}\right|$und nach Korollar 7.14 schließlich mit $T$. Wir können also $J e_{j}=e_{j}$ annehmen. Die $e_{j}$ bilden eine Orthonormalbasis von $\mathcal{K}$; daher kann man das interessierende Skalarprodukt zwischen $f^{ \pm} \in \mathcal{L}^{ \pm}(r)$ und $k \in Q(E) \mathcal{K}$ so entwickeln:

$$
\begin{aligned}
\left\langle f^{ \pm} \mid k\right\rangle=\left\langle P_{\mathcal{L}}^{ \pm}(r) f^{ \pm} \mid Q(E) \omega^{-\frac{1}{2}} \omega^{+\frac{1}{2}} k\right\rangle & =\left\langle f^{ \pm} \mid T^{ \pm *} \omega^{\frac{1}{2}} k\right\rangle \\
& =\sum_{j}\left\langle f^{ \pm} \mid e_{j}\right\rangle\left\langle e_{j} \mid T^{ \pm *} \omega^{\frac{1}{2}} k\right\rangle=\sum_{j}\left\langle f^{ \pm} \mid e_{j}\right\rangle\left\langle\omega^{\frac{1}{2}} T^{ \pm} e_{j} \mid k\right\rangle .
\end{aligned}
$$

\footnotetext{
${ }^{4}$ Siehe dazu Anhang 7.2.D.
} 
(Man beachte, daß das Bild von $T^{ \pm}$nach Definition (7.2.27) immer im Definitionsbereich von $\omega^{\frac{1}{2}}$ liegt.) Im Sinne schwacher Konvergenz gilt also

$$
Q(E) P_{\mathcal{L}}^{ \pm}(r)=\sum_{j} Q(E)\left|\omega^{\frac{1}{2}} T^{ \pm} e_{j}\right\rangle\left\langle e_{j}\right| P_{\mathcal{L}}^{ \pm}(r)
$$

Wir wissen dabei, daß

$$
\left\|T^{ \pm} e_{j}\right\|^{2}=\left\langle\left. e_{j}|| T^{ \pm}\right|^{2} e_{j}\right\rangle \leq\left\langle e_{j} \mid T^{2} e_{j}\right\rangle=t_{j}^{2} \leq E^{-1} \cdot(E r)^{2 \alpha} \cdot \text { const } .
$$

Es sei noch einmal darauf hingewiesen, daß mit den $T^{ \pm}(E, r)$ auch $T$ und die $e_{j}$ explizit von $E$ und $r$ abhängen.

\subsubsection{Aufspaltung der Weyloperatoren}

Wir übertragen die bewiesenen bzw. vorausgesetzten Eigenschaften der Theorie im Einteilchenraum jetzt auf den Fockraum, indem wir eine Reihenentwicklung für Weyloperatoren $W(f)$ herleiten.

Sei dazu $f \in \mathcal{L}(r)$. Wir zerlegen $f$ wie in (7.1.1) in "Real- und Imaginärteil", d.h. wir schreiben $f=f^{+}+i f^{-}$mit $J$-invarianten Vektoren $f^{ \pm} \in \mathcal{L}^{ \pm}(r)$. Der Weyloperator $W(f)$ kann dann wie folgt als Exponentialreihe dargestellt werden:

$$
\begin{aligned}
W(f)= & e^{-\frac{1}{2}\|f\|^{2}} e^{i a^{*}(f)} e^{i a(f)}=e^{-\frac{1}{2}\|f\|^{2}} e^{i a^{*}\left(f^{+}\right)} e^{-a^{*}\left(f^{-}\right)} e^{i a\left(f^{+}\right)} e^{+a\left(f^{-}\right)} \\
& =e^{-\frac{1}{2}\|f\|^{2}} \sum_{m^{ \pm}, n^{ \pm} \in \mathbb{N}_{0}} \frac{i^{m^{+}+n^{+}+2 m^{-}}}{m^{+} ! m^{-} ! n^{+} ! n^{-} !} a^{*}\left(f^{+}\right)^{m^{+}} a^{*}\left(f^{-}\right)^{m^{-}} a\left(f^{+}\right)^{n^{+}} a\left(f^{-}\right)^{n^{-}} .
\end{aligned}
$$

Die Gleichungen sind dabei im Sinne quadratischer Formen auf $\mathcal{H}^{0} \times \mathcal{H}^{0}$ zu verstehen. Um unsere Kenntnisse über die Theorie im Einteilchenraum einbringen zu können, müssen wir die vorkommenden Polynome von Erzeugungs- und Vernichtungsoperatoren in Operatoren zwischen den $n$-Teilchen-Räumen zerlegen.5 Der Übersichtlichkeit halber beginnen wir mit den einfachsten Monomen:

Lemma 7.3. Im Sinne von Operatoren von $\mathcal{H}^{0}$ nach $\mathcal{H}^{0}$ gilt

$$
a^{*}\left(f^{ \pm}\right)=\sum_{w=0}^{\infty} \sqrt{w+1} \operatorname{Symm}\left(\left|f^{ \pm}\right\rangle \otimes \mathbf{1}_{w}\right), \quad a\left(f^{ \pm}\right)=\sum_{w=0}^{\infty} \sqrt{w+1} \operatorname{Symm}\left(\left\langle f^{ \pm}\right| \otimes \mathbf{1}_{w}\right) .
$$

(Dabei ist $\mathbf{1}_{w}$ der Einsoperator auf $\mathcal{H}_{w}$; die Operatoren unter der Symmetrisierung sind durch 0 auf das orthogonale Komplement von $\mathcal{H}_{w}$ bzw. $\mathcal{H}_{w+1}$ fortgesetzt.)

Beweis. Zunächst sieht man unmittelbar, daß die formal unendlichen Summen über $w$ nach Anwendung auf einen Vektor aus $\mathcal{H}^{0}$ endlich werden und wieder einen Vektor aus

\footnotetext{
${ }^{5}$ Das dazu verwendete Konzept ist ein Spezialfall einer allgemeinen Entwicklungsformel für beschränkte Operatoren auf dem Fockraum [Ära63, section 6]. Wir benötigen den allgemeinen Formalismus hier jedoch nicht.
} 
$\mathcal{H}^{0}$ liefern. Aus Gründen der Linearität reicht es, die Relationen auf Vektoren der Form $\operatorname{Symm}\left(b_{1} \otimes \cdots \otimes b_{k}\right) \in \mathcal{H}_{k}$ nachzuprüfen. Dort hat man

$$
\begin{aligned}
& \sum_{w=0}^{\infty} \sqrt{w+1} \operatorname{Symm}\left(\left|f^{ \pm}\right\rangle \otimes \mathbf{1}_{w}\right) \operatorname{Symm}\left(b_{1} \otimes \cdots \otimes b_{k}\right) \\
& \quad=\sqrt{k+1} \operatorname{Symm}\left(f^{ \pm} \otimes b_{1} \otimes \cdots \otimes b_{k}\right) \stackrel{(7.1 .10)}{=} a^{*}\left(f^{ \pm}\right) \operatorname{Symm}\left(b_{1} \otimes \cdots \otimes b_{k}\right) .
\end{aligned}
$$

Damit ist die Relation für Erzeuger gezeigt; diejenige für Vernichter folgt analog.

In höheren Monomen $a^{*}\left(f^{+}\right)^{m^{+}} a^{*}\left(f^{-}\right)^{m^{-}} a\left(f^{+}\right)^{n^{+}} a\left(f^{-}\right)^{n^{-}}$können wir nun jeden Faktor nach Lemma 7.3 entwickeln; die auftretenden Mehrfachsummen reduzieren sich dabei sofort auf eine einzelne Summation, denn es ist

$$
\operatorname{Symm}\left(\left|f^{+}\right\rangle \otimes \mathbf{1}_{w}\right) \cdot \operatorname{Symm}\left(\left|f^{+}\right\rangle \otimes \mathbf{1}_{w^{\prime}}\right)=\delta_{w, w^{\prime}+1} \operatorname{Symm}\left(\left|f^{+}\right\rangle^{\otimes 2} \otimes \mathbf{1}_{w}\right)
$$

und so weiter. Man erhält auf diese Weise:

Lemma 7.4. Im Sinne von Operatoren von $\mathcal{H}^{0}$ nach $\mathcal{H}^{0}$ gilt

$$
\begin{aligned}
a^{*}\left(f^{+}\right)^{m^{+}} a^{*}\left(f^{-}\right)^{m^{-}} a\left(f^{+}\right)^{n^{+}} a\left(f^{-}\right)^{n^{-}} & \\
& =\sum_{w=0}^{\infty} \frac{\sqrt{(m+w) !(n+w) !}}{w !} \operatorname{Symm}\left(\stackrel{m^{+}}{\otimes}\left|f^{+}\right\rangle \stackrel{m^{-}}{\otimes}\left|f^{-}\right\rangle \stackrel{n^{+}}{\otimes}\left\langle\left. f^{+}\right|^{n^{-}} \otimes\left\langle f^{-}\right| \otimes \mathbf{1}_{w}\right) .\right.
\end{aligned}
$$

Dabei ist $m=m^{+}+m^{-}, n=n^{+}+n^{-}$.

Wenn wir die unter der Symmetrisierung auftretenden Einteilchen-Vektoren und - Linearformen noch zusätzlich mit einer Energiebeschränkung versehen, dann können wir die Reihenentwicklung aus Eigenschaft 7.1 einsetzen. So ergibt sich

$$
\begin{aligned}
\otimes Q(E)\left(\stackrel{m^{+}}{\otimes}\left|f^{+}\right\rangle \stackrel{m^{-}}{\otimes}\left|f^{-}\right\rangle \stackrel{n^{+}}{\otimes}\left\langle f^{+}\right| \stackrel{n^{-}}{\otimes}\left\langle f^{-}\right|\right) \otimes Q(E) \\
=\sum_{k_{i}^{ \pm}, l_{j}^{ \pm} \in \mathbb{N}} \prod\left\langle f^{+} \mid h_{k_{i}^{+}}^{+}\right\rangle \prod\left\langle f^{-} \mid h_{k_{i}^{-}}^{-}\right\rangle \prod\left\langle f^{+} \mid h_{l_{j}^{+}}^{+}\right\rangle \prod\left\langle f^{-} \mid h_{l_{j}^{-}}^{-}\right\rangle \\
\quad \times \otimes Q(E)\left(\otimes\left|g_{k_{i}^{+}}^{+}\right\rangle \otimes\left|g_{k_{i}^{-}}^{-}\right\rangle \otimes\left\langle g_{l_{j}^{+}}^{+}\right| \otimes\left\langle g_{l_{j}^{-}}^{-}\right|\right) \otimes Q(E) .
\end{aligned}
$$

- hier wird über $m^{+}+m^{-}+n^{+}+n^{-}$verschiedene Indizes summiert. Wir haben verwendet, daß sowohl die $f^{ \pm}$wie die $h_{k}^{ \pm}$invariant unter $J$ sind, so daß im Skalarprodukt die Seiten vertauscht werden können. Eine entsprechende Entwicklung erhält man auch nach (7.2.29) mit den $e_{j}$.

Wir setzen dieses Ergebnis nun in den Ausdruck aus Lemma 7.4 ein; da die Summation über $w$ in Matrixelementen tatsächlich immer endlich ist, läßt sich die Reihenfolge der Summenzeichen ohne weiteres vertauschen. Wir wenden dann Lemma 7.4 erneut an; das ergibt:

$$
\begin{aligned}
& P(E) a^{*}\left(f^{+}\right)^{m^{+}} a^{*}\left(f^{-}\right)^{m^{-}} a\left(f^{+}\right)^{n^{+}} a\left(f^{-}\right)^{n^{-}} P(E) \\
& =\sum_{k_{i}^{ \pm}, l_{j}^{+} \in \mathbb{N}} \prod\left\langle f^{+} \mid h_{k_{i}^{+}}^{+}\right\rangle \prod\left\langle f^{-} \mid h_{k_{i}^{-}}^{-}\right\rangle \prod\left\langle f^{+} \mid h_{l_{j}^{+}}^{+}\right\rangle \prod\left\langle f^{-} \mid h_{l_{j}^{-}}^{-}\right\rangle \\
& \quad \times P(E) \prod a^{*}\left(g_{k_{i}^{+}}^{+}\right) \prod a^{*}\left(g_{k_{i}^{-}}^{-}\right) \prod a\left(g_{l_{j}^{+}}^{+}\right) \prod a\left(g_{l_{j}^{-}}^{-}\right) P(E) .
\end{aligned}
$$


Um aus diesen Termen nach (7.2.31) Weyloperatoren zu erhalten, muß noch über $m^{ \pm}$und $n^{ \pm}$summiert werden. Dazu organisieren wir die Summe um, indem wir

- alle Terme mit gleichen Potenzen von $\left\langle f^{+} \mid h_{j}^{+}\right\rangle$und $\left\langle f^{-} \mid h_{j}^{-}\right\rangle$zusammenfassen und

- diese Summanden mit zwei Multiindizes $\mu^{ \pm} \in \mathfrak{M}^{\infty}$ numerieren, wobei $\mu_{1}^{+}$die Anzahl der Faktoren $\left\langle f^{+} \mid h_{1}^{+}\right\rangle$zählt usf.

Wir werden $\mu^{+}$und $\mu^{-}$manchmal auch zu einem einzigen Multiindex $\mu$ zusammenfassen, wobei wir die Anordnung der Komponenten $\mu_{j}$ so wählen, daß das analog zu (7.2.14) gebildete $\vartheta(j)$ monoton mit $j$ wächst.

Auf diese Weise erhält man schließlich

$$
P(E) W(f) P(E)=\sum_{\mu^{+}, \mu^{-}} e^{-\frac{1}{2}\|f\|^{2}}\left\langle f^{+} \mid h^{+}\right\rangle^{\mu^{+}}\left\langle f^{-} \mid h^{-}\right\rangle^{\mu^{-}} \cdot P(E) \phi_{\mu^{+} \mu^{-}} P(E)
$$

mit Operatoren (quadratischen Formen) $\phi_{\mu^{+} \mu^{-}}$, die sich ergeben als

$$
\phi_{\mu^{+} \mu^{-}}=\sum \frac{i^{m^{+}+n^{+}+2 m^{-}}}{m^{+} ! m^{-} ! n^{+} ! n^{-} !} a^{*}\left(g_{?}\right) \ldots a\left(g_{?}\right) \ldots
$$

$a^{*}\left(g_{?}\right) \ldots a\left(g_{\text {? }}\right) \ldots$ sind gewisse Produkte von Erzeugern bzw. Vernichtern der $g_{j}^{ \pm}$, und zwar mit der durch die Multiindizes $\mu^{+}, \mu^{-}$beschriebenen Multiplizität. Summiert wird über alle möglichen solchen Produkte; insgesamt enthält die Summe $\frac{\left|\mu^{+}\right| !\left|\mu^{-}\right| !}{\mu^{+} ! \mu^{-} !}\left(\left|\mu^{+}\right|+1\right)\left(\left|\mu^{-}\right|+1\right)$ Terme. $]^{6}$ Die Entwicklung (7.2.36) konvergiert im Sinne quadratischer Formen auf $\mathcal{H}^{0} \times \mathcal{H}^{0}$.

Analog kommt man zu einer Entwicklung

$$
P(E) W(f) P(E)=\sum_{\mu^{+}, \mu^{-}} e^{-\frac{1}{2}\|h\|^{2}}\left\langle f^{+} \mid e\right\rangle^{\mu^{+}}\left\langle f^{-} \mid e\right\rangle^{\mu^{-}} \cdot P(E) \chi_{\mu^{+} \mu^{-}} P(E)
$$

mit Operatoren $\chi_{\mu^{+} \mu^{-}}$, die aus entsprechenden Summen mit Erzeugern und Vernichtern $\operatorname{der} \omega^{+\frac{1}{2}} T^{ \pm} e_{j}$ gebildet werden .

\subsubsection{Erweiterung und Normkonvergenz der Reihe}

Wir wollen die angegebenen Entwicklungen (7.2.36) und (7.2.38) jetzt auf ganz $\mathfrak{A}(r)$ ausdehnen;] hierzu müssen einerseits die Normen der einzelnen Summanden abgeschätzt werden, andererseits müssen die von $f^{ \pm}$abhängigen Vorfaktoren durch lineare Funktionale der $W(f)$ ersetzt werden.

Wir betrachten zunächst die Operatoren $P(E) \phi_{\mu^{+} \mu^{-}} P(E)$, die eine Summendarstellung wie in (7.2.37) besitzen. Unter Verwendung sogenannter „Energieschranken“ [BP90, sect. $3.3]$

$$
\left\|P(E) \prod_{j=1}^{J} a^{*}\left(\omega^{\frac{1}{2}} b_{j}\right) \prod_{k=1}^{K} a\left(\omega^{\frac{1}{2}} \hat{b}_{k}\right) P(E)\right\| \leq \prod_{j=1}^{J}\left\|b_{j}\right\| \prod_{k=1}^{K}\left\|\hat{b}_{k}\right\| E^{\frac{J+K}{2}} ; \quad b_{j}, \hat{b}_{k} \in \mathcal{K}
$$

\footnotetext{
${ }^{6}$ Bei vorgegebenem $\mu^{+}$müssen $m^{+}$und $n^{+}$so gewählt werden, daß $m^{+}+n^{+}=\left|\mu^{+}\right|$; dafür gibt es $\left(\left|\mu^{+}\right|+1\right)$ Möglichkeiten. Sind $m^{+}, n^{+}$fixiert, dann hat man noch die Einträge von $\mu^{+}$auf die einzelnen Erzeuger/Vernichter zu „,verteilen“, wofür es per Multinomialkoeffizienten $\left|\mu^{+}\right| ! / \mu^{+}$! Alternativen gibt. $\mu^{-}$ liefert einen analogen Beitrag.

${ }^{7}$ Für den Ausdruck (7.2.36) wird das nur teilweise gelingen.
} 
sieht man unmittelbar, daß ihre einzelnen Summanden beschränkt sind; außerdem ist die Schranke für jeden Summanden gleich, da sie sich bei Permutation der $g_{j}^{ \pm}$nicht ändert. $\mathrm{Zu}$ den Vorfaktoren in (7.2.37) sei bemerkt, daß stets $m^{ \pm}+n^{ \pm}=\left|\mu^{ \pm}\right|$und daher $\left|\mu^{ \pm}\right| ! /\left(m^{ \pm} ! n^{ \pm} !\right) \leq 2^{\left|\mu^{ \pm}\right|}$(Binomialkoeffizienten). Damit ergibt sich

$$
\begin{aligned}
\left\|\phi_{\mu}\right\|_{E}=\| & P(E) \phi_{\mu} P(E) \| \\
\leq \frac{2^{|\mu|}}{\mu !}\left(\left|\mu^{+}\right|+1\right)\left(\left|\mu^{-}\right|+1\right)\left\|Q(E) \omega^{-\frac{1}{2}} g^{+}\right\|^{\mu^{+}} & \left\|Q(E) \omega^{-\frac{1}{2}} g^{-}\right\|^{\mu^{-}} E^{\frac{|\mu|}{2}} \\
& \leq \frac{4^{|\mu|}}{\mu !}\left\|E^{\frac{1}{2}} Q(E) \omega^{-\frac{1}{2}} g\right\|^{\mu} .
\end{aligned}
$$

Wir haben dabei sehr grob $\left|\mu^{ \pm}\right|+1 \leq 2^{\left|\mu^{ \pm}\right|}$abgeschätzt. Entsprechend erhält man unter Verwendung von (7.2.30):

$$
\left\|\chi_{\mu}\right\|_{E}=\left\|P(E) \chi_{\mu} P(E)\right\| \leq \frac{4^{|\mu|}}{\mu !} t^{\mu^{+}} t^{\mu^{-}} E^{\frac{|\mu|}{2}} .
$$

Nun werden die von $f^{ \pm}$abhängigen Faktoren in (7.2.36) betrachtet. Wir nutzen dazu die in Anhang 7.2.B konstruierten speziellen Funktionale aus. Nach Lemma 7.8 lassen sich die fraglichen Faktoren als Auswertung eines Funktionals $\sigma_{\mu^{+} \mu^{-}}$auf $W(f)$ darstellen:

$$
\sigma_{\mu^{+} \mu^{-}}(W(f))=e^{-\frac{1}{2}\|f\|^{2}}\left\langle f^{+} \mid h^{+}\right\rangle^{\mu^{+}}\left\langle f^{-} \mid h^{-}\right\rangle^{\mu^{-}} .
$$

Wir können also diese Funktionale in Formel (

$$
P(E) W(f) P(E)=\sum_{\mu} \sigma_{\mu}(W(f)) \cdot P(E) \phi_{\mu} P(E)
$$

und mit analog (nach Lemma 7.9) gebildeten $\tau_{\mu}$ zur Entwicklung (7.2.38)

$$
P(E) W(f) P(E)=\sum_{\mu} \tau_{\mu}(W(f)) \cdot P(E) \chi_{\mu} P(E) .
$$

Beide Entwicklungen lassen sich sofort auf die lineare Hülle der $W(f)$ fortsetzen. Um eine stetige Fortsetzung auf ganz $\mathfrak{A}(r)$ zu ermöglichen, muß noch die Normkonvergenz der obigen Reihen gezeigt werden; wegen der schwachen Stetigkeit der Funktionale $\sigma_{\mu}$ bzw. $\tau_{\mu}$ reicht es zu zeigen, daß die Reihe der Normen konvergiert. Nach Lemma 7.8 und 7.9 wissen wir, daß

$$
\left\|\sigma_{\mu^{+} \mu^{-}}\right\| \leq 2^{|\mu|} \sqrt{|\mu| !}\left\|P_{\mathcal{L}}^{+}(r) h^{+}\right\|^{\mu^{+}}\left\|P_{\mathcal{L}}^{-}(r) h^{-}\right\|^{\mu^{-}} \quad ; \quad\left\|\tau_{\mu^{+} \mu^{-}}\right\| \leq 4^{|\mu|} \sqrt{\mu !} .
$$

Nur im letzteren Fall reichen diese Schranken aus, um die gewünschte Konvergenz zu etablieren: Man erhält

$$
\sum_{\mu^{+}, \mu^{-}}\left\|\tau_{\mu^{+} \mu^{-}}\right\|\left\|\chi_{\mu^{+} \mu^{-}}\right\|_{E} \leq \sum_{\mu^{+}, \mu^{-}} 16^{\left|\mu^{+}\right|+\left|\mu^{-}\right|} \frac{t^{\mu^{+}+\mu^{-}}}{\sqrt{\mu^{+} ! \mu^{-!}}} E^{\frac{|\mu|}{2}}=\left(\prod_{j=1}^{\infty} \sum_{k=0}^{\infty} \frac{\left(16 E^{\frac{1}{2}} t_{j}\right)^{k}}{\sqrt{k !}}\right)^{2} .
$$


Die Potenzreihe

$$
F(z)=\sum_{k=0}^{\infty} \frac{(16 z)^{k}}{\sqrt{k !}}
$$

konvergiert für alle $z \in \mathbb{C}$ nach dem Quotientenkriterium; insbesondere konvergiert die Reihe in (7.2.46). Zur Konvergenz des unendlichen Produkts beachte man, daß $\log F(z)$ eine differenzierbare Funktion ist mit $\log F(0)=0, \log F(x)>0 \forall x>0$; daher gibt zu $x_{0}>0$ eine Konstante $c^{\prime}$, so daß

$$
0 \leq \log F(x) \leq c^{\prime} \cdot x \quad \forall x \in\left[0, x_{0}\right] .
$$

Da wegen Eigenschaft 7.2 gilt

$$
E^{\frac{1}{2}} t_{j} \leq E^{\frac{1}{2}}\|T\|_{1} \leq \text { const. }
$$

können wir $x_{0}$ dabei unabhängig von $E$ so wählen, daß stets $E^{\frac{1}{2}} t_{j} \leq x_{0}$. Mit diesen Abschätzungen wird

$$
\log \prod_{f=1}^{\infty} F\left(E^{\frac{1}{2}} t_{j}\right)=\sum_{j=1}^{\infty} \log F\left(E^{\frac{1}{2}} t_{j}\right) \leq c^{\prime} \sum_{j=1}^{\infty} E^{\frac{1}{2}} t_{j}=c^{\prime} \cdot E^{\frac{1}{2}} \operatorname{tr} T \leq \text { const. }
$$

Die Konvergenz der Summe (7.2.44) ist also gewährleistet, und zwar gleichmäßig im Argument von $\tau_{\mu}$; wir haben im Sinne der Normkonvergenz von Abbildungen

$$
\Xi_{\mathrm{R} E, r}=\sum_{\mu} \tau_{\mu} \cdot P(E) \chi_{\mu} P(E) .
$$

Für die Entwicklung nach $\sigma_{\mu}, \phi_{\mu}$ läßt sich aufgrund der schlechteren Abschätzungen für die $\left\|\sigma_{\mu}\right\|$ keine derartige Konvergenz für die gesamte Reihe zeigen - der Faktor $\sqrt{|\mu| !}$ statt $\sqrt{\mu !}$ in $(7.2 .45)$ verhindert dies. Man kann jedoch die Teilsummen von (7.2.43) bei festen Teilchenzahlen $\left|\mu^{ \pm}\right|$betrachten; wegen $m^{ \pm}+n^{ \pm}=\left|\mu^{ \pm}\right|$in (7.2.35) kann man auch sie auf die lineare Hülle der $W(f)$ fortsetzen. Für die Summe der Normen gilt

$$
\begin{gathered}
\sum_{\left|\mu^{ \pm}\right|=N^{ \pm}}\left\|\sigma_{\mu^{+} \mu^{-}}\right\|\left\|\phi_{\mu^{+} \mu^{-}}\right\|_{E} \\
\leq \sum_{\left|\mu^{ \pm}\right|=N^{ \pm}} 8^{|\mu|} \frac{\sqrt{|\mu| !}}{\mu !}\left\|P_{\mathcal{L}}^{+}(r) h^{+}\right\|^{\mu^{+}}\left\|P_{\mathcal{L}}^{-}(r) h^{-}\right\|^{\mu^{-}}\left\|E^{\frac{1}{2}} Q(E) \omega^{-\frac{1}{2}} g^{+}\right\|^{\mu^{+}}\left\|E^{\frac{1}{2}} Q(E) \omega^{-\frac{1}{2}} g^{-}\right\|^{\mu^{-}} \\
\leq 8^{N^{+}+N^{-}} \sqrt{\left(N^{+}+N^{-}\right) !} \prod_{j=1}^{\infty} \sum_{k=0}^{\infty} \frac{\left(y_{j}^{+}\right)^{k}}{k !} \cdot \prod_{j=1}^{\infty} \sum_{k=0}^{\infty} \frac{\left(y_{j}^{-}\right)^{k}}{k !} \\
\operatorname{mit} \\
y_{j}^{ \pm}:=\left\|P_{\mathcal{L}}^{ \pm}(r) h_{j}^{ \pm}\right\|\left\|E^{\frac{1}{2}} Q(E) \omega^{-\frac{1}{2}} g_{j}^{ \pm}\right\| . \quad(7.2 .52)
\end{gathered}
$$

Wir haben dabei die Summation - nach Herausziehen der Vorfaktoren - wieder auf alle Multiindizes ausgedehnt und die Reihe ähnlich wie in (7.2.46) umgeformt. Für die verbleibenden unendliche Summen und Produkte gilt

$$
\prod_{j=1}^{\infty} \sum_{k=0}^{\infty} \frac{\left(y_{j}^{ \pm}\right)^{k}}{k !}=\prod_{j=1}^{\infty} \exp \left(y_{j}^{ \pm}\right)=\exp \left(\sum_{j=1}^{\infty} y_{j}^{ \pm}\right) \leq \exp \left((c E r)^{\vartheta^{ \pm}(1)} \cdot \text { const. }\right) \stackrel{(E r \leq 1)}{\leq} \text { const.' }
$$


Dies ist nach Eigenschaft 7.1 sichergestellt. Die betrachteten Teilsummen konvergieren damit gleichmäßig auf $\mathfrak{A}(r)$. Wegen $|\mu|=\left|\mu^{+}\right|+\left|\mu^{-}\right|$konvergiert für $N_{0} \in \mathbb{N}$ dann auch

$$
\sum_{|\mu| \leq N_{0}} \sigma_{\mu} \cdot P(E) \phi_{\mu} P(E)
$$

in der Operatornorm.

Für die uns interessierende Anwendung benötigen wir noch weitere Abschätzungen für die Normen gewisser Teilsummen der beiden Reihenentwicklungen. Dabei beginnen wir wieder mit der Entwicklung nach $\tau_{\mu}$ und $\chi_{\mu}$ aus (7.2.44). Wir fixieren ein $j \in \mathbb{N}$ und ein Vorzeichen „土“ und betrachten nur die Summanden, in denen der $j$-te Eintrag des Multiindex $\mu^{ \pm}$mindestens einmal besetzt ist:

$$
\begin{aligned}
& \sum_{\mu: \mu_{j}^{ \pm} \geq 1}\left\|\tau_{\mu}\right\|\left\|\chi_{\mu}\right\|_{E} \leq \sum_{\mu: \mu_{j}^{ \pm} \geq 1} 16^{|\mu|} \frac{\left(E^{\frac{1}{2}} t\right)^{\mu^{+}+\mu^{-}}}{\sqrt{\mu !}} \\
& \leq 16 E^{\frac{1}{2}} t_{j} \cdot \sum_{\mu} 16^{|\mu|} \frac{\left(E^{\frac{1}{2}} t\right)^{\mu^{+}+\mu^{-}}}{\sqrt{\mu !}} \leq E^{\frac{1}{2}} t_{j} \cdot \text { const. }
\end{aligned}
$$

Dabei wird im letzten Schritt wieder über alle $\mu$ summiert; die Nenner der Summanden wurden nach unten abgeschätzt. Wir folgern für die Summe über alle an irgendeiner Stelle mindestens einmal besetzten Multiindizes:

$$
\begin{array}{r}
\sum_{|\mu| \geq 1}\left\|\tau_{\mu}\right\|\left\|\chi_{\mu}\right\|_{E} \leq \sum_{ \pm} \sum_{j=1}^{\infty} \sum_{\mu: \mu_{j}^{ \pm} \geq 1} 16^{|\mu|} \frac{\left(E^{\frac{1}{2}} t\right)^{\mu^{+}+\mu^{-}}}{\sqrt{\mu !}} \stackrel{(\sqrt{7.255})}{\leq} 2 \sum_{j=1}^{\infty} E^{\frac{1}{2}} t_{j} \cdot \text { const. } \\
=2 E^{\frac{1}{2}}\|T\|_{1} \cdot \text { const. } \leq(\text { Er })^{\alpha} \cdot \text { const.' }
\end{array}
$$

nach Eigenschaft 7.2. Nun ergibt sich für die Summe über alle mindestens $N_{0}$-fach besetzten Indizes $\left(N_{0} \in \mathbb{N}\right)$ :

$$
\begin{aligned}
\sum_{|\mu| \geq N_{0}}\left\|\tau_{\mu}\right\|\left\|\chi_{\mu}\right\|_{E} \leq & \sum_{|\mu| \geq N_{0}} 16^{|\mu|} \frac{\left(E^{\frac{1}{2}} t\right)^{\mu^{+}+\mu^{-}}}{\sqrt{\mu !}} \\
& \leq\left(\sum_{|\mu| \geq 1} 16^{|\mu|} \frac{\left(E^{\frac{1}{2}} t\right)^{\mu^{+}+\mu^{-}}}{\sqrt{\mu !}}\right)^{N_{0}} \stackrel{(\sqrt{7.2 .56})}{\leq}(E r)^{N_{0} \alpha} \cdot \operatorname{const} .\left(N_{0}\right)
\end{aligned}
$$

denn durch Ausmultiplizieren der Potenz $(\ldots)^{N_{0}}$ ergeben sich höchstens mehr als die gewünschten Terme; im Nenner muß wieder abgeschätzt werden.

Ähnliches berechnen wir jetzt für die Entwicklung nach den $\sigma_{\mu}$ und $\chi_{\mu}$ laut (7.2.43) bzw. (7.2.54). Die Summen konvergieren dabei stets nur, wenn wir die Summation auf $|\mu| \leq N_{0}$ einschränken; diese Bedingung notieren wir am Summenzeichen als $\sum^{*}$. Alle im folgenden auftretenden Konstanten hängen von $N_{0}$ ab, was aber nicht explizit notiert wird. Mit denselben Prinzipien wie in (7.2.55) erhalten wir für $j, m \in \mathbb{N}$ :

$$
\sum_{\mu: \mu_{j} \geq m}^{*}\left\|\sigma_{\mu}\right\|\left\|\phi_{\mu}\right\|_{E} \stackrel{\sqrt{7.2 .522}}{\leq} \sum_{\mu: \mu_{j} \geq m}^{*} \frac{y^{\mu}}{\mu !} \cdot \text { const. } \leq y_{j}^{m} \sum_{\mu}^{*} \frac{y^{\mu}}{\mu !} \cdot \text { const } \leq y_{j}^{m} \cdot \text { const.' }
$$


Wichtig ist für unsere Zwecke noch die Summation über alle $\mu$, die für gegebenes $j_{0} \in$ $\mathbb{N}$ mindestens eine Besetzung $\mu_{j} \geq 1$ für ein $j \geq j_{0}$ aufweisen. Man erhält als obere Abschätzung

$$
\sum_{j=j_{0}}^{\infty} \sum_{\mu: \mu_{j} \geq 1}^{*}\left\|\sigma_{\mu}\right\|\left\|\phi_{\mu}\right\|_{E} \stackrel{(7.2 .58)}{\leq} \sum_{j=j_{0}}^{\infty} y_{j} \cdot \text { const. } \leq(\text { Er })^{\vartheta\left(j_{0}\right)} \cdot \text { const. }^{\prime}\left(j_{0}\right)
$$

unter Verwendung der Normschranken in Eigenschaft 7.1.

\subsubsection{Anwendung auf die Abbildung $\Xi$}

Wir werden nun die bisherigen Ergebnisse über Reihenentwicklungen der Abbildung $\Xi_{\mathrm{R} E, r}$ zusammenfassen und sie schließlich zur Approximation der Abbildung $\Xi: \bar{\Sigma} \times \overline{\mathfrak{A}} \rightarrow \mathbb{C}$ verwenden.

Die für diesen Zweck gewünschten (weil skalenunabhängigen) Approximationsterme sind die $\sigma_{\mu} \phi_{\mu}$; wir konnten die Konvergenz der Reihe über diese Terme aber oben nicht etablieren. Geht man jedoch zurück zu (7.2.31) und (7.2.35), dann sieht man, daß für jeden Wert der $m^{ \pm}, n^{ \pm}$die gewählte Art der Entwicklung der Einteilchenraum-Skalarprodukte unabhängig gewählt werden kann. Wegen $m^{ \pm}+n^{ \pm}=\left|\mu^{ \pm}\right|$kann man insbesondere die Terme zu kleinen Teilchenzahlen nach Abschnitt 7.2.2, diejenigen zu hohen Teilchenzahlen nach Abschnitt 7.2.3 entwickeln. Man erhält so eine „gemischte“ Entwicklung

$$
\Xi_{\mathrm{R} E, r}=\sum_{|\mu| \leq N_{0}} \sigma_{\mu} \cdot P(E) \phi_{\mu} P(E)+\sum_{|\mu|>N_{0}} \tau_{\mu} \cdot P(E) \chi_{\mu} P(E),
$$

die nach obigen Überlegungen in der Normtopologie konvergiert. $N_{0}$ kann hier beliebig festgesetzt werden.

Wir interessieren uns dabei vor allem für die Normschranken der Rang-1-Terme $\sigma_{\mu} \phi_{\mu}$ : Nach (7.2.26) haben wir

$$
\left\|\sigma_{\mu}\right\|_{r}\left\|\phi_{\mu}\right\|_{E} \leq(E r)^{\Theta(\mu)} \cdot \text { const. }
$$

mit der Festsetzung

$$
\Theta(\mu):=\sum_{j} \mu_{j} \vartheta(j)
$$

Im folgenden werden wir die $\sigma_{\mu}$ auch als $\sigma_{l}$ mit natürlichen Zahlen $l$ numerieren (analog die $\phi_{l}$ ). Dabei soll das aus (7.2.62) resultierende $\Theta(l)$ monoton wachsen, was wegen $\vartheta(j)>0$, $\vartheta(j) \rightarrow \infty(j \rightarrow \infty)$ möglich ist. Es gilt sogar $\Theta(l) \rightarrow \infty(l \rightarrow \infty)$.

Wesentlich ist nun, daß wir die $\phi_{l} \sigma_{l}$ als ,skalenunabhängige“ Rang-1-Terme aus $\bar{\Psi}_{0}^{P}$ ansehen können: Wegen der in Eigenschaft 7.1 genannten „Verträglichkeitsbedingung“ sind die $\phi_{l}$ wohldefinierte Linearformen auf $\bar{\Sigma}$, und die polynomialen Energieschranken folgen aus (7.2.26) und (7.2.40). Die Konstruktion der $\sigma_{l}$ können wir etwa bei $r=r_{0}$ fixieren, ohne ihre Einschränkungen auf $\mathfrak{A}(r)\left(r \leq r_{0}\right)$ zu ändern; wegen $\sigma_{l} \in \Sigma$ erfüllen die $\phi_{l} \sigma_{l}$ dann die für Elemente von $\bar{\Psi}_{0}^{P}$ geforderten Stetigkeitsbedingungen. 
Es sei nun $\bar{\gamma}>0$ gegeben; wir wollen zeigen, daß

$$
\gamma\left(\Xi-\sum_{l=1}^{N} \phi_{l} \sigma_{l}\right) \geq \bar{\gamma}
$$

für genügend große $N$. Dazu wählen wir $N$ derart, daß $\Theta(N+1) \geq \bar{\gamma}$. Weiter sei $N_{0}$ so groß, daß $N_{0} \alpha>\bar{\gamma}$ und $j_{0}$ groß genug, damit $\vartheta\left(j_{0}\right)>\bar{\gamma}$. Für festes $E \geq E_{0}, r \leq r_{0}, E r \leq 1$ erhalten wir dann

$$
\left\|\Xi-\sum_{l=1}^{N} \phi_{l} \sigma_{l}\right\|_{E, r} \leq \sum_{|\mu| \leq N_{0}}^{\diamond}\left\|\phi_{\mu}\right\|_{E} \cdot\left\|\sigma_{\mu}\right\|_{r}+\sum_{|\mu|>N_{0}}\left\|\chi_{\mu}\right\|_{E} \cdot\left\|\tau_{\mu}\right\| .
$$

Dabei fehlen in der Summe $\sum^{\diamond}$ alle Terme zu $l \leq N$, d.h. die zu den Summanden der Reihe korrespondierenden Multiindizes $\mu$ erfüllen $\Theta(\mu) \geq \bar{\gamma}$. Wir spalten nun davon noch eine weitere Summe $\sum^{\infty} \mathrm{ab}$, die nur über solche Terme läuft, die $\mu_{j}=0 \forall j \geq j_{0}$ erfüllen. (Diese Summe ist wegen $|\mu| \leq N_{0}$ sicher endlich.) Wir können dann den ersten Summanden in (7.2.64) so abschätzen:

$$
\begin{aligned}
\sum_{|\mu| \leq N_{0}}^{\diamond}\left\|\phi_{\mu}\right\|_{E}\left\|\sigma_{\mu}\right\|_{r} \leq \sum_{|\mu| \leq N_{0}}^{\infty}\left\|\phi_{\mu}\right\|_{E}\left\|\sigma_{\mu}\right\|_{r}+\sum_{j=j_{0}}^{\infty} \sum_{\mu: \mu_{j} \geq 1}^{*}\left\|\phi_{\mu}\right\|_{E}\left\|\sigma_{\mu}\right\|_{r} \\
\leq(E r)^{\bar{\gamma}} \cdot \text { const. }+(E r)^{\vartheta\left(j_{0}\right)} \cdot \text { const }^{\prime} \leq(E r)^{\bar{\gamma}} \cdot \text { const." }
\end{aligned}
$$

Wir haben dabei die Abschätzung (7.2.59) verwendet. Den zweiten Summanden in (7.2.64) können wir mit Hilfe von (7.2.57) kontrollieren. Damit erhalten wir insgesamt

$$
\left\|\Xi-\sum_{l=1}^{N} \phi_{l} \sigma_{l}\right\|_{E, r} \leq(E r)^{\bar{\gamma}} \cdot \text { const. }
$$

und zwar zunächst für $E \geq E_{0}, r \leq r_{0}$ und $E r \leq 1$. Da aber $\|\cdot\|_{E, r}$ mit $E$ sicher anwächst, gilt für beliebige $E \geq 1$ :

$$
\left\|\Xi-\sum_{l=1}^{N} \phi_{l} \sigma_{l}\right\|_{E, r} \leq\left\|\Xi-\sum_{l=1}^{N} \phi_{l} \sigma_{l}\right\|_{E \cdot E_{0}, r} \leq E_{0}^{\bar{\gamma}}(E r)^{\bar{\gamma}} \cdot \text { const. }
$$

für $E r \leq E_{0}^{-1}$ und $r \leq r_{0}$. Der Faktor $E_{0}^{\bar{\gamma}}$ kann in die Konstante absorbiert werden. Wir haben nun für kleine $w: 8$

$$
\sup _{E r \leq w}\left\|\Xi-\sum_{l=1}^{N} \phi_{l} \sigma_{l}\right\|_{E, r} \leq w^{\bar{\gamma}} \cdot \text { const. }
$$

und folglich

$$
\gamma\left(\Xi-\sum_{l=1}^{N} \phi_{l} \sigma_{l}\right) \geq \bar{\gamma}
$$

Unser Ergebnis können wir also (unter geringfügiger Änderung der Bezeichnungsweisen) so formulieren:

\footnotetext{
${ }^{8}$ Es sei daran erinnert, daß das Supremum per Definition nur über $E \geq 1$ läuft, so daß kleine Werte von $w$ auch solche von $r$ implizieren.
} 
Satz 7.5. Wir betrachten ein Modell der freien Feldtheorie, das die Eigenschaften 7.1 und 7.9 besitzt, etwa die Theorie eines reellen skalaren Feldes in $s \geq 3$ Raumdimensionen. Dann gibt es Rang-1-Abbildungen $\phi_{j} \sigma_{j} \in \bar{\Psi}_{0}^{P}$, so daß

$$
\Xi=\sum_{j=1}^{\infty} \phi_{j} \sigma_{j}
$$

im Sinne der von $\delta(\cdot)$ auf $\bar{\Psi}$ gelieferten Topologie. Das asymptotische Phasenraumkriterium mit polynomialen Energieschranken ist erfüllt. Genauer hat man für jedes $N \in \mathbb{N}$ :

$$
\gamma\left(\Xi-\sum_{l=1}^{N} \phi_{l} \sigma_{l}\right) \geq \Theta(N+1) .
$$

In Abschnitt 7.4 .2 werden die ersten Terme der berechneten Reihe für das reelle skalare Feld explizit angeben; die $\phi_{j}$ lassen sich dort mit Funktionen des der Theorie zugrundeliegenden Punktfeldes $\phi(x)$ identifizieren.

\subsection{A Multiindex-Schreibweise}

Um im Zusammenhang mit der freien Feldtheorie die Notation zu verkürzen, ist es an mehreren Stellen sinnvoll, Multiindizes zu verwenden. Wir geben hier einen Überblick über die wichtigsten damit gebildeten Kurzschreibweisen.

Unter einem n-stelligen Multiindex verstehen wir ein $n$-Tupel $\kappa \in\left(\mathbb{N}_{0}\right)^{n}$. Eine Folge $\mu=\left(\mu_{i}\right)_{i=1}^{\infty}$ in $\mathbb{N}_{0}$ wird als Multiindex beliebiger Stellenzahl bezeichnet, vorausgesetzt, daß nur endlich viele $\mu_{i}$ von Null verschieden sind. Die Menge aller $n$-stelligen Multiindizes bezeichnen wir mit $\mathfrak{M}^{n}$, die aller Multiindizes beliebiger Stellenzahl mit $\mathfrak{M}^{\infty}$. Die Zahl

$$
|\kappa|:=\sum_{i=1}^{n} \kappa_{i}
$$

heißt Länge des Multiindex; bei beliebiger Stellenzahl läuft die Summe formal bis $\infty$, ist aber tatsächlich endlich.

Die Mengen $\mathfrak{M}^{n}$ und $\mathfrak{M}^{\infty}$ versehen wir mit einer natürlichen (Halb-)Ordnungsstruktur, indem wir für Multiindizes $\kappa, \kappa^{\prime}$ setzen

$$
\kappa \geq \kappa^{\prime} \quad: \Leftrightarrow \quad \kappa_{j} \geq \kappa_{j}^{\prime} \forall j
$$

Analog werden die Relationen ", $\leq$, , ,> und , $<$ " definiert.

Sei $\kappa$ ein $n$-stelliger Multiindex und $x \in \mathbb{R}^{n}$, dann schreiben wir

$$
x^{\kappa}:=x_{1}^{\kappa_{1}} \cdot \ldots \cdot x_{n}^{\kappa_{n}} .
$$

In ähnlicher Weise kürzen wir Differentialoperatoren ab:

$$
\partial_{\kappa}:=\frac{\partial^{\kappa_{1}}}{\partial x_{1}^{\kappa_{1}}} \ldots \frac{\partial^{\kappa_{n}}}{\partial x_{n}^{\kappa_{n}}} .
$$


Addition von Multiindizes sowie die Multiplikation mit Zahlen aus $\mathbb{N}_{0}$ werden komponentenweise erklärt. Die Fakultät eines $n$-stelligen Multiindex $\kappa$ ist

$$
\kappa !:=\prod_{j=1}^{n} \kappa_{j} !,
$$

entsprechend für Multiindizes beliebiger Stellenzahl.

Wir werden auch diverse andere, suggestive Schreibweisen mit Multiindizes verwenden. Sind beispielsweise $b_{1} \ldots b_{n} \in \mathcal{K}$ und wieder $\kappa \in \mathfrak{M}^{n}$, dann notieren wir:

$$
\begin{gathered}
b^{\otimes \kappa}=\underbrace{b_{1} \otimes \ldots \otimes b_{1}}_{\kappa_{1}} \otimes \ldots \otimes \underbrace{b_{n} \otimes \ldots \otimes b_{n}}_{\kappa_{n}} ; \\
\|b\|^{\kappa}=\prod_{j=1}^{n}\left\|b_{j}\right\|^{\kappa_{j}} ; \\
\langle b \mid f\rangle^{\kappa}=\prod_{j=1}^{n}\left\langle b_{j} \mid f\right\rangle^{\kappa_{j}} \quad(f \in \mathcal{K}) .
\end{gathered}
$$

\subsection{B Spezielle Funktionale}

Für unsere Analyse der freien Feldtheorie im Fockraum benötigen wir spezielle lineare Funktionale, die auf Weyloperatoren $W(f)$ bestimmte vorgegebene Werte annehmen. Wir konstruieren sie mit Hilfe einer erzeugenden Funktion, um die auftretenden kombinatorischen Probleme zu umgehen. Für die Anwendung ist es weiterhin wichtig, die Normen der besagten Funktionale kontrollieren zu können; daher beginnen wir mit der Abschätzung der Normen gewisser Vektoren im Fockraum.

Lemma 7.6. Seien $b_{j} \in \mathcal{K}(j \in \mathbb{N}), \kappa$ ein Multiindex und

$$
\psi=a\left(b_{?}\right) \ldots a^{*}\left(b_{?}\right) \ldots \Omega
$$

wobei $a\left(b_{?}\right) \ldots$ für gewisse Produkte der Vernichter der $b_{j}$ steht (analog $a^{*}\left(b_{?}\right) \ldots$ ) und $\kappa_{j}$ dabei die Multiplizität von $b_{j}$ zählt (ohne Unterschied, ob als Erzeuger oder Vernichter). Dann gilt

$$
\|\psi\| \leq \sqrt{|\kappa| !}\|b\|^{\kappa}
$$

Bilden die $b_{j}$ ein Orthonormalsystem, dann folgt sogar

$$
\|\psi\| \leq \sqrt{\kappa !} .
$$

Beweis. Wir verwenden Induktion nach der Anzahl $n$ der Vernichter. Für $n=0$ ist

$$
\psi=a^{*}(b)^{\kappa} \Omega=\sqrt{|\kappa| !} \operatorname{Symm}\left(b^{\otimes \kappa}\right)
$$

und damit

$$
\|\psi\|^{2} \leq|\kappa| !\left\|b^{\otimes \kappa}\right\|^{2}=|\kappa| !\|b\|^{2 \kappa} .
$$


Bilden die $b_{j}$ ein Orthonormalsystem, dann hat man explizit

$$
\left\|\operatorname{Symm}\left(b^{\otimes \kappa}\right)\right\|^{2}=\frac{\kappa !}{|\kappa| !}
$$

womit der Induktionsanfang gezeigt ist.

Nun nehmen wir ohne Einschränkung an, daß in $\psi$ ein Vernichter $a\left(b_{1}\right)$ vorkommt. Weiter fixieren wir eine Reihenfolge der vorkommenden Erzeuger und numerieren sie als $a^{*}\left(b_{(k)}\right)$. Dann wird

$$
\begin{gathered}
\|\psi\|=\left\|a\left(b_{?}\right) \ldots a\left(b_{1}\right) a^{*}\left(b_{?}\right) \ldots \Omega\right\|=\|a\left(b_{?}\right) \ldots \sum_{k}\left\langle b_{1} \mid b_{(k)}\right\rangle \underbrace{a^{*}\left(b_{?}\right) \ldots \Omega \|}_{\text {ohne } b_{(k)}}\| \\
\leq \sum_{k}\left\|b_{1}\right\|\left\|b_{(k)}\right\|\|a\left(b_{?}\right) \ldots \underbrace{a^{*}\left(b_{?}\right) \ldots}_{\text {ohne } b_{(k)}} \Omega\| \\
\quad \stackrel{\text { (I.V.) }}{\leq}(|\kappa|-1) \sqrt{(|\kappa|-2) !}\|b\|^{\kappa} \leq \sqrt{|\kappa| !}\|b\|^{\kappa} .
\end{gathered}
$$

Im Fall des Orthonormalsystems ergibt sich nur dann ein Beitrag von $\left\langle b_{1} \mid b_{(k)}\right\rangle$, wenn $b_{(k)}=$ $b_{1}$, und man erhält einen Faktor $\kappa_{1}-1$ statt $|\kappa|-1$.

Als Folgerung ergibt sich:

Lemma 7.7. Seien $b_{j} \in \mathcal{K}(j \in \mathbb{N})$, $\kappa$ ein Multiindex und $\tau \in \mathfrak{B}(\mathcal{H})_{*}$ das Funktional

$$
\tau(A)=\left(a\left(b_{?}\right) \ldots a^{*}\left(b_{?}\right) \ldots \Omega \mid A a\left(b_{?}\right) \ldots a^{*}\left(b_{?}\right) \ldots \Omega\right),
$$

wobei wieder $\kappa_{j}$ die Multiplizität von $b_{j}$ zählt (ohne Unterschied, auf welcher Seite oder ob als Erzeuger oder Vernichter). Dann gilt

$$
\|\tau\| \leq \sqrt{|\kappa| !}\|b\|^{\kappa}
$$

Bilden die $b_{j}$ ein Orthonormalsystem, dann folgt sogar

$$
\|\tau\| \leq \sqrt{\kappa !}
$$

Beweis. Wir teilen $\kappa$ auf in $\kappa_{L}+\kappa_{R}$, die jeweils die Besetzungszahlen auf der linken bzw. rechten Seite beschreiben. Die Norm von $\tau$ wird durch die Norm der Vektoren im Skalarprodukt abgeschätzt, und Lemma 7.6 liefert

$$
\|\tau\| \leq \sqrt{\left|\kappa_{L}\right| !\left|\kappa_{R}\right| !}|| b\left\|^{\kappa} \leq \sqrt{|\kappa| !}\right\| b \|^{\kappa} .
$$

Im Fall des Orthonormalsystems gilt entsprechendes, wobei man $\kappa_{L} ! \kappa_{R} ! \leq \kappa$ ! beachtet.

Nun kommen wir zur Konstruktion der bereits erwähnten Funktionale mit vorgegebenen Werten auf Weyloperatoren. Es seien $k<m \in \mathbb{N}$ und $b_{1}, \ldots, b_{m} \in \mathcal{K}$. Man betrachte folgendes Funktional:

$$
\tau(A):=\left(\Omega \mid\left[a\left(b_{1}\right),\left[\ldots \left[a\left(b_{k}\right),\left[a^{*}\left(b_{k+1}\right),\left[\ldots\left[a^{*}\left(b_{m}\right), A\right] \ldots\right] \Omega\right) .\right.\right.\right.\right.
$$


Durch Ausrechnen der Kommutatoren erhält man eine Darstellung der Form

$$
\tau(A)=\sum_{j}(-1)^{j}\left(a\left(b_{?}\right) \ldots a^{*}\left(b_{1}\right) \ldots a^{*}\left(b_{k}\right) \Omega \mid A a^{*}\left(b_{?}\right) \ldots \Omega\right),
$$

wobei jeweils einige der $b_{k+1}, \ldots, b_{m}$ auf der linken, die anderen auf der rechten Seite des Skalarprodukts stehen (angedeutet durch $b_{\text {? }}$ ) und die Summe über alle möglichen Aufteilungen läuft, insgesamt über $2^{m-k}$ Terme. Lemma 7.7 liefert dann

$$
\|\tau\| \leq 2^{m} \sqrt{m !} \prod_{j}\left\|b_{j}\right\| .
$$

Entscheidend ist, daß sich der Wert von $\tau$ auf Weyloperatoren $W(f)$ leicht ausrechnen läßt: Mit den Relationen (7.1.14) und (7.1.15) erhält man unmittelbar

$$
\tau(W(f))=e^{-\frac{1}{2}\|f\|^{2}} \prod_{j=1}^{k}\left\langle b_{j} \mid i f\right\rangle \prod_{j=k+1}^{m}\left\langle i f \mid b_{j}\right\rangle .
$$

Wir gehen nun zu dem Fall über, in dem die $b_{j}$ auch mehrfach in den Kommutatoren auftreten können; zu Multiindizes $\mu, \nu \in \mathfrak{M}^{\infty}$ („Besetzungszahlen“) können wir dann Funktionale $\tau_{\mu \nu} \in \mathfrak{B}(\mathcal{H})_{*}$ finden, so daß

$$
\tau_{\mu \nu}(W(f))=e^{-\frac{1}{2}\|f\|^{2}} \prod_{j}\left\langle b_{j} \mid i f\right\rangle^{\mu_{j}} \prod_{k}\left\langle i f \mid b_{k}\right\rangle^{\nu_{k}} .
$$

Für ihre Norm gilt

$$
\left\|\tau_{\mu \nu}\right\| \leq 2^{|\mu|+|\nu|} \sqrt{(|\mu|+|\nu|) !}\|b\|^{\mu+\nu} .
$$

Falls die $b_{j}$ ein Orthonormalsystem bilden, liefert Lemma 7.7 eine bessere Abschätzung:

$$
\left\|\tau_{\mu \nu}\right\| \leq 2^{|\mu|+|\nu|} \sqrt{(\mu+\nu) !} .
$$

Es erweist sich als nützlich, die Funktionale $\tau_{\mu \nu}$ aus einer erzeugenden Funktion zu gewinnen: Sei $f \in \mathcal{K}$ fest; man setze

$$
G(s, t):=e^{-\frac{1}{2}\|f\|^{2}} \exp \left(\sum_{j}\left\langle b_{j} \mid i f\right\rangle s_{j}+\sum_{k}\left\langle i f \mid b_{k}\right\rangle t_{k}\right), s, t \in \mathbb{C}^{n} \text { (n geeignet), }
$$

dann gilt

$$
\tau_{\mu \nu}(W(f))=\left.\frac{\partial^{\mu}}{\partial s^{\mu}} \frac{\partial^{\nu}}{\partial t^{\nu}} G(s, t)\right|_{s=0, t=0} .
$$

Wir zerlegen $f$ nun wie in (7.1.1) als $f=f^{+}+i f^{-}$mit $J f^{ \pm}=f^{ \pm}$. Weiter nehmen wir an, daß auch die $b_{j}$ invariant unter $J$ sind. Dann können wir $G(s, t)$ folgendermaßen umschreiben:

$$
\begin{aligned}
G(s, t):=e^{-\frac{1}{2}\|f\|^{2}} & \exp \left(\sum_{j}\left(i\left\langle b_{j} \mid f^{+}\right\rangle-\left\langle b_{j} \mid f^{-}\right\rangle\right) s_{j}+\sum_{k}\left(-i\left\langle b_{k} \mid f^{+}\right\rangle-\left\langle b_{k} \mid f^{-}\right\rangle\right) t_{k}\right) \\
= & e^{-\frac{1}{2}\|f\|^{2}} \exp (\sum_{j}\left\langle b_{j} \mid f^{+}\right\rangle \underbrace{\left(i s_{j}-i t_{j}\right.}_{=: u_{j}})+\sum_{k}\left\langle b_{k} \mid f^{-}\right\rangle \underbrace{\left(-s_{k}-t_{k}\right)}_{=: w_{k}}) .
\end{aligned}
$$


Die Ableitung nach den „neuen Variablen“ $u$ und $w$ liefert

$$
\left.\frac{\partial^{\mu}}{\partial u^{\mu}} \frac{\partial^{\nu}}{\partial w^{\nu}} G(u, w)\right|_{u=0, w=0}=e^{-\frac{1}{2}\|h\|^{2}} \prod_{j}\left\langle b_{j} \mid f^{+}\right\rangle^{\mu_{j}} \prod_{k}\left\langle b_{k} \mid f^{-}\right\rangle^{\nu_{k}}=: \sigma_{\mu \nu}(W(f)) .
$$

Andererseits lassen sich die Ableitung nach neuen und alten Variablen leicht durcheinander ausdrücken: Man hat offenbar

$$
\frac{\partial}{\partial u_{i}}=-\frac{i}{2} \frac{\partial}{\partial s_{i}}+\frac{i}{2} \frac{\partial}{\partial t_{i}}, \quad \frac{\partial}{\partial w_{i}}=-\frac{1}{2} \frac{\partial}{\partial s_{i}}-\frac{1}{2} \frac{\partial}{\partial t_{i}}
$$

da die numerischen Faktoren konstant sind, erhält man

$$
\frac{\partial^{\mu}}{\partial u^{\mu}} \frac{\partial^{\nu}}{\partial w^{\nu}}=\left(-\frac{i}{2} \frac{\partial}{\partial s}+\frac{i}{2} \frac{\partial}{\partial t}\right)^{\mu}\left(-\frac{1}{2} \frac{\partial}{\partial s}-\frac{1}{2} \frac{\partial}{\partial t}\right)^{\nu}=\sum_{\mu^{\prime}, \nu^{\prime}} c_{\mu^{\prime} \nu^{\prime}} \frac{\partial^{\mu^{\prime}}}{\partial s^{\mu^{\prime}}} \frac{\partial^{\nu^{\prime}}}{\partial t^{\nu^{\prime}}}
$$

mit Konstanten $c_{\mu^{\prime} \nu^{\prime}}$ vom Betrag $2^{-|\mu|-|\nu|}$; summiert wird über solche $\mu^{\prime}, \nu^{\prime}$, die $\mu^{\prime}+\nu^{\prime}=$ $\mu+\nu$ erfüllen (eventuell kommt ein solches Paar $\mu^{\prime}, \nu^{\prime}$ mehrfach vor); insgesamt hat die Summe $2^{|\mu|+|\nu|}$ Summanden. Dies bedeutet, daß man die Funktionale $\sigma_{\mu \nu}$ auf ganz $\mathfrak{B}(\mathcal{H})$ erweitern kann; sie ergeben sich als Linearkombinationen der $\tau_{\mu \nu}$ :

$$
\sigma_{\mu \nu}=\sum_{\mu^{\prime}, \nu^{\prime}} c_{\mu^{\prime} \nu^{\prime}} \tau_{\mu^{\prime} \nu^{\prime}}
$$

Mit der bereits bemerkten Abschätzung der Vorfaktoren erhält man

$$
\left\|\sigma_{\mu \nu}\right\| \leq 2^{|\mu|+|\nu|} \sqrt{(|\mu|+|\nu|) !}\|b\|^{\mu+\nu} \quad \text { bzw. } \quad\left\|\sigma_{\mu \nu}\right\| \leq 2^{|\mu|+|\nu|} \sqrt{(\mu+\nu) !}
$$

(letzteres im Fall eines Orthonormalsystems).

Wir können durch Umdefinition der Multiindizes $\mu, \nu$ auch erreichen, daß in den Skalarprodukten $\left\langle\cdot \mid f^{+}\right\rangle$und $\left\langle\cdot \mid f^{-}\right\rangle$die Funktionen $b_{j}$ unabhängig voneinander gewählt werden können. Somit erhält man:

Lemma 7.8. Seien $\mu^{+}, \mu^{-}$Multiindizes beliebiger Stellenzahl, $b_{j}^{ \pm} \in \mathcal{K}$ mit $J b_{j}^{ \pm}=b_{j}^{ \pm}$. Dann gibt es ein schwach stetiges Funktional $\sigma_{\mu^{+} \mu^{-}} \in \mathfrak{B}(\mathcal{H})_{*}$, so daß

$$
\sigma_{\mu^{+} \mu^{-}}(W(f))=e^{-\frac{1}{2}\|f\|^{2}}\left\langle b^{+} \mid f^{+}\right\rangle^{\mu^{+}}\left\langle b^{-} \mid f^{-}\right\rangle^{\mu^{-}} \quad \forall f \in \mathcal{K} .
$$

Es gilt

$$
\left\|\sigma_{\mu^{+} \mu^{-}}\right\| \leq 2^{\left|\mu^{+}\right|+\left|\mu^{-}\right|} \sqrt{\left(\left|\mu^{+}\right|+\left|\mu^{-}\right|\right) !}\left\|b^{+}\right\|^{\mu^{+}}\left\|b^{-}\right\|^{\mu^{-}} .
$$

Für ein Orthonormalsystem lautet das Ergebnis so:[]

Lemma 7.9. Seien $\mu^{+}, \mu^{-}$Multiindizes beliebiger Stellenzahl, $b_{j} \in \mathcal{K}$ orthonormal mit $J b_{j}=b_{j}$. Dann gibt es ein schwach stetiges Funktional $\sigma_{\mu^{+} \mu^{-}} \in \mathfrak{B}(\mathcal{H})_{*}$, so daß

$$
\sigma_{\mu^{+} \mu^{-}}(W(f))=e^{-\frac{1}{2}\|f\|^{2}}\left\langle b \mid f^{+}\right\rangle^{\mu^{+}}\left\langle b \mid f^{-}\right\rangle^{\mu^{-}} \quad \forall f \in \mathcal{K} .
$$

Es gilt

$$
\left\|\sigma_{\mu^{+} \mu^{-}}\right\| \leq 4^{\left|\mu^{+}\right|+\left|\mu^{-}\right|} \sqrt{\mu^{+} ! \mu^{-} !} .
$$

\footnotetext{
${ }^{9}$ Man verwendet dabei noch die Abschätzung $\frac{(k+l) !}{k ! l !}=\left(\begin{array}{c}k+l \\ k\end{array}\right) \leq 2^{k+l}$.
} 
Wir wollen noch ein weiteres Detail der konstruierten Funktionale fixieren: Falls die Einteilchenraumvektoren $b_{j}$ in $(7.2 .83)$ energiebeschränkt sind, etwa $b_{j} \in Q(E) \mathcal{K}$, dann überträgt sich dies auf die Hilbertraum-Vektoren $\psi=a\left(b_{\text {? }}\right) \ldots a^{*}\left(b_{\text {? }}\right) \ldots \Omega$; sie liegen in $P(n E) \mathcal{H}$, wenn insgesamt $n$ Erzeuger auftreten. Für die Funktionale $\sigma_{\mu^{+} \mu^{-}}$ergibt sich so:

Korollar 7.10. Liegen in Lemma 7.8 oder 7.9 die $b_{j} b z w . b_{j}^{ \pm}$in $Q(E) \mathcal{K}$, dann gilt

$$
\sigma_{\mu^{+} \mu^{-}} \in \Sigma(\hat{E}) \quad \text { mit } \quad \hat{E}=\left(\left|\mu^{+}\right|+\left|\mu^{-}\right|\right) E .
$$

\subsection{Spurnorm der Operatoren $T^{ \pm}(E, r)$ für das reelle skalare freie Feld}

In diesem Abschnitt soll gezeigt werden, daß für das reelle skalare freie Feld die Einteilchenraum-Operatoren

$$
T^{ \pm}(E, r)=Q(E) \omega^{-\frac{1}{2}} P_{\mathcal{L}}^{ \pm}(r)
$$

im Fall $s \geq 3$ in der Spurklasse sind, und das Verhalten ihrer Spurnormen mit $E$ und $r$ soll untersucht werden. Dabei verwenden wir das folgende, bereits in [BW86] angewandte Verfahren: Kann man $T^{ \pm}$als Produkt von vier Operatoren schreiben,

$$
T^{ \pm}=A \cdot B \cdot C \cdot D
$$

wobei $A$ und $D$ beschränkt, $B$ und $C$ Hilbert-Schmidt-Operatoren sind, dann ist $T^{ \pm}$Spuroperator, ${ }^{10}$ und für seine Spurnorm gilt

$$
\left\|T^{ \pm}\right\|_{1} \leq\|A\| \cdot\|B\|_{2} \cdot\|C\|_{2} \cdot\|D\| .
$$

Der Vorteil dieser Vorgehensweise liegt darin, daß sich die Hilbert-Schmidt-Norm für sogenannte Integralkernoperatoren leicht berechnen läßt (s.u.).

Die konkrete Aufspaltung von $T^{ \pm}$wird so konstruiert: Wir fixieren eine Testfunktion $\chi(\vec{x}) \in \mathcal{S}\left(\mathbb{R}^{s}\right)$, die $\chi(\vec{x})=1$ für $|\vec{x}| \leq 1$ erfüllt, und setzen $\chi_{r}(\vec{x})=\chi\left(r^{-1} \vec{x}\right)$. Die Funktionen $\chi_{r}$ wirken durch Multiplikation als Operatoren „im Ortsraum“. Nach Definition der Räume $\mathcal{L}^{ \pm}(r)$ hat man nun die Identität

$$
\omega^{\mp \frac{1}{2}} \chi_{r} \chi_{r} \omega^{ \pm \frac{1}{2}} P_{\mathcal{L}}^{ \pm}(r)=P_{\mathcal{L}}^{ \pm}(r)
$$

- das ist zumindest auf einer dichten Menge sofort klar und läßt sich dann stetig fortsetzen. Wir erhalten daraus folgende Zerlegung der $T^{ \pm}$:

$$
\begin{aligned}
T^{ \pm}(E, r)=\underbrace{\left(Q(E) \omega^{\alpha}\left(1+r^{2} \omega^{2}\right)^{2 s}\right)}_{A} \cdot \underbrace{\left(\left(1+r^{2} \omega^{2}\right)^{-2 s} \omega^{-\alpha-\frac{1}{2} \mp \frac{1}{2}} \chi_{r}\left(1+r^{2} \omega^{2}\right)^{s}\right)}_{B} \\
\cdot \underbrace{\left(\left(1+r^{2} \omega^{2}\right)^{-s} \chi_{r} \omega^{ \pm \frac{1}{2}}\right)}_{C} \cdot \underbrace{P_{\mathcal{L}}^{ \pm}(r)}_{D} .
\end{aligned}
$$

\footnotetext{
${ }^{10}$ Das Produkt zweier Hilbert-Schmidt-Operatoren liegt stets in der Spurklasse, und diese ist ein beidseitiges Ideal in $\mathfrak{B}(\mathcal{H})$.
} 
Dabei ist $\alpha$ eine zunächst unbestimmte positive Zahl; wir werden später sehen, welche Werte dafür zulässig sind. Man berechnet

$$
\|A\|=\left\|Q(E) \omega^{\alpha}\left(1+r^{2} \omega^{2}\right)^{2 s}\right\| \leq E^{\alpha}\left(1+(E r)^{2}\right)^{2 s} \leq E^{\alpha} \cdot \text { const. } \quad(E r \leq 1) ;
$$

außerdem ist natürlich $\|D\|=\left\|P_{\mathcal{L}}^{ \pm}(r)\right\|=1$.

Bei $B$ und $C$ handelt es sich, im Impulsraum betrachtet, um Integralkernoperatoren, d.h. sie sind von der Form

$$
(B f)(\vec{p})=\int K_{B}(\vec{p}, \vec{q}) f(\vec{q}) d^{s} q
$$

mit einer Funktion $K_{B}(\vec{p}, \vec{q})$, dem Integralkern (analog für $C$ ). Die Hilbert-Schmidt-Norm solch eines Operators läßt sich berechnen als

$$
\|B\|_{2}^{2}=\int\left|K_{B}(\vec{p}, \vec{q})\right|^{2} d^{s} p d^{s} q
$$

und $B$ liegt genau dann in der Hilbert-Schmidt-Klasse, wenn dieser Ausdruck konvergiert. Tatsächlich erhält man die Integralkerne von $B$ und $C$ so: Transformiert man den Operator $\chi_{r}$ in den Impulsraum, dann wirkt er, wie man durch Ausschreiben der Fouriertransformation sieht, als

$$
\left(\chi_{r} f\right)(\vec{p})=(2 \pi)^{-\frac{s}{2}} \int \tilde{\chi}_{r}(\vec{p}-\vec{q}) f(\vec{q}) d^{s} q
$$

wobei $\tilde{\chi}_{r}$ die Fouriertransformierte von $\chi_{r}$ bezeichnet. Da die übrigen Anteile von $B$ bzw. $C$ durch Multiplikation im Impulsraum wirken, erhält man als Integralkerne:

$$
\begin{aligned}
& K_{B}(\vec{p}, \vec{q})=(2 \pi)^{-\frac{s}{2}}\left(1+r^{2} \vec{p}^{2}+r^{2} m^{2}\right)^{-2 s}\left(\vec{p}^{2}+m^{2}\right)^{-\frac{\alpha}{2}-\frac{1}{4} \mp \frac{1}{4}} \tilde{\chi}_{r}(\vec{p}-\vec{q})\left(1+r^{2} \vec{q}^{2}+r^{2} m^{2}\right) \\
& K_{C}(\vec{p}, \vec{q})=(2 \pi)^{-\frac{s}{2}}\left(1+r^{2} \vec{p}^{2}+r^{2} m^{2}\right)^{-s} \tilde{\chi}_{r}(\vec{p}-\vec{q})\left(\vec{q}^{2}+m^{2}\right)^{ \pm \frac{1}{4}}
\end{aligned}
$$

Bevor wir zur Abschätzung der Hilbert-Schmidt-Normen kommen, beweisen wir noch eine Hilfsformel: Es gilt

$$
\frac{\vec{q}^{2}+c^{2}}{\vec{p}^{2}+c^{2}} \leq\left(\frac{|\vec{p}-\vec{q}|}{c}+1\right)^{2} \quad \text { für } \vec{p}, \vec{q} \in \mathbb{R}^{s}, c>0 .
$$

Offenbar reicht es durch Skalierung der Vektoren, die Formel für $c=1 \mathrm{zu}$ beweisen; wir schreiben $\vec{d}=\vec{q}-\vec{p}$ und erhalten

$$
\frac{\vec{q}^{2}+1}{\vec{p}^{2}+1}=\frac{(\vec{p}+\vec{d})^{2}+1}{\vec{p}^{2}+1}=\frac{\vec{p}^{2}+\vec{d}^{2}+2 \vec{p} \vec{d}+1}{\vec{p}^{2}+1} \leq 1+2 \underbrace{\frac{|\vec{p}|}{\vec{p}^{2}+1}}_{\leq 1}|\vec{d}|+|\vec{d}|^{2} \leq(1+|\vec{d}|)^{2} .
$$


Nun wenden wir uns der Hilbert-Schmidt-Norm von $B \mathrm{zu}$; unter Verwendung von

$$
\tilde{\chi}_{r}(\vec{p})=r^{s} \tilde{\chi}(r \vec{p})
$$

erhält man

$$
\begin{aligned}
& \|B\|_{2}^{2}=(2 \pi)^{-s} r^{2 s} \int d^{s} p d^{s} q\left(\frac{1+r^{2} \vec{q}^{2}+r^{2} m^{2}}{1+r^{2} \vec{p}^{2}+r^{2} m^{2}}\right)^{2 s} \frac{\left(\vec{p}^{2}+m^{2}\right)^{-\alpha-\frac{1}{2} \mp \frac{1}{2}}}{\left(1+r^{2} \vec{p}^{2}+r^{2} m^{2}\right)^{s}}|\tilde{\chi}(r(\vec{p}-\vec{q}))|^{2} \\
& \stackrel{\text { (Skalierung) }_{=}}{=}(2 \pi)^{-s} r^{2 \alpha+1 \pm 1} \int d^{s} p d^{s} q\left(\frac{1+\vec{q}^{2}+r^{2} m^{2}}{1+\vec{p}^{2}+r^{2} m^{2}}\right)^{2 s} \frac{\left(\vec{p}^{2}+r^{2} m^{2}\right)^{-\alpha-\frac{1}{2} \mp \frac{1}{2}}}{\left(1+\vec{p}^{2}+r^{2} m^{2}\right)^{s}}|\tilde{\chi}(\vec{p}-\vec{q})|^{2} \\
& \stackrel{(\pi .2 .108)}{\leq}(2 \pi)^{-s} r^{2 \alpha+1 \pm 1} \int d^{s} p d^{s}(p-q) \underbrace{(1+|\vec{p}-\vec{q}|)^{2 s}|\tilde{\chi}(\vec{p}-\vec{q})|^{2}}_{\text {(I) }} \cdot \underbrace{\frac{1}{\left(1+\vec{p}^{2}\right)^{s}|\vec{p}|^{2 \alpha+1 \pm 1}}}_{\text {(II) }} .
\end{aligned}
$$

Dabei haben wir im letzten Schritt $r m \geq 0$ abgeschätzt. Da $\chi$ Testfunktion ist, existiert das Integral über (I) in $(\vec{p}-\vec{q})$. Auch dasjenige in $\vec{p}$ (Term II) existiert, solange $|\vec{p}|^{-2 \alpha-1 \mp 1}$ bei $\vec{p}=0$ integrabel bleibt; das erfordert

$$
2 \alpha+1 \pm 1<s \quad \Leftrightarrow \quad \alpha<\frac{s-1 \mp 1}{2} .
$$

Wir setzen $s \geq 3$ voraus, damit solche (positiven) $\alpha$ existieren. Es gilt dann

$$
\|B\|_{2} \leq r^{\alpha+\frac{1}{2} \pm \frac{1}{2}} \cdot \text { const. }
$$

Für den Operator $C$ gehen wir analog vor:

$$
\begin{gathered}
\|C\|_{2}^{2}=(2 \pi)^{-s} r^{2 s} \int d^{s} p d^{s} q\left(\frac{1}{1+r^{2} \vec{p}^{2}+r^{2} m^{2}}\right)^{2 s}|\tilde{\chi}(r(\vec{p}-\vec{q}))|^{2}\left(\vec{q}^{2}+m^{2}\right)^{ \pm \frac{1}{2}} \\
\stackrel{\text { (Skalierung) }}{=}(2 \pi)^{-s} r^{\mp 1} \int d^{s} p d^{s} q\left(\frac{1+\vec{q}^{2}+r^{2} m^{2}}{1+\vec{p}^{2}+r^{2} m^{2}}\right)^{2 s}|\tilde{\chi}(\vec{p}-\vec{q})|^{2} \frac{\left(\vec{q}^{2}+r^{2} m^{2}\right)^{ \pm \frac{1}{2}}}{\left(1+\vec{q}^{2}+r^{2} m^{2}\right)^{2 s}} \\
\frac{(7.2 .108)}{\leq}(2 \pi)^{-s} r^{\mp 1} \int d^{s}(p-q) d^{s} q \underbrace{(1+|\vec{p}-\vec{q}|)^{2 s}|\tilde{\chi}(\vec{p}-\vec{q})|^{2}}_{\text {(I) }} \cdot \underbrace{\frac{\left(\vec{q}^{2}+r^{2} m^{2}\right)^{ \pm \frac{1}{2}}}{\left(1+\vec{q}^{2}\right)^{2 s}}}_{\text {(II) }}
\end{gathered}
$$

Wieder existiert das Integral über $(\vec{p}-\vec{q})$. Im Term (II) schätzen wir $r^{2} m^{2}$ im Fall „_-“ gegen $0 \mathrm{ab}$, im Fall „+" setzen wir $m r \leq 1$ voraus; dann erhält man auch für das Integral über $q$ eine $r$-unabhängige Abschätzung, denn für $s \geq 2$ bleibt $|\vec{q}|^{-1}$ lokal integrabel. Man hat also insgesamt

$$
\|C\|_{2} \leq r^{\mp \frac{1}{2}} \cdot \text { const. }
$$

Fassen wir die Ergebnisse nach (7.2.99) zusammen, dann ergibt sich

$$
\left\|T^{ \pm}\right\|_{1} \leq r^{\frac{1}{2}}(E r)^{\alpha} \cdot \text { const. für } \alpha<\frac{s-1 \mp 1}{2}, r m \leq 1 .
$$

Die Konstante hängt allerdings von $\alpha$ ab. Nach Umbenennung von $\alpha$ können wir folgende Aussage über die Operatoren $T^{ \pm}(E, r)$ formulieren: 
Satz 7.11. Wir betrachten die Theorie eines reellen skalaren freien Feldes in $s \geq 3$ Raumdimensionen. Für $E r \leq 1, r \leq \frac{1}{m}(r<\infty$ im Fall $m=0)$ sind die Operatoren $T^{ \pm}(E, r)$ in der Spurklasse; ist $0<\alpha<\frac{s-1}{2}$, dann gibt es eine Konstante c, so daß

$$
\begin{aligned}
& \left\|T^{+}\right\|_{1} \leq c \cdot E^{-\frac{1}{2}}(E r)^{\alpha}, \\
& \left\|T^{-}\right\|_{1} \leq c \cdot E^{-\frac{1}{2}}(E r)^{\alpha+1} .
\end{aligned}
$$

Insbesondere besitzt die betrachtete Theorie die Eigenschaft 7.9.

Die hier etablierten Abschätzungen lassen sich allerdings im Fall niedriger Raum-ZeitDimensionen $(s \leq 2)$ nicht mehr aufrechterhalten, da dann Integrale wie (7.2.111) nicht mehr gleichmäßig im Kurzabstandslimes abzuschätzen sind. Dieses singuläre Verhalten der niederdimensionalen Theorien wurde bereits von Buchholz und Porrmann bemerkt; explizit weiß man, daß im Fall $s=2, m=0$ der Operator $T^{+}$nicht in der Spurklasse liegt [BP90].

Es soll allerdings bemerkt werden, daß sich die Abschätzungen in leicht veränderter Form immerhin auf die $2+1$-dimensionale massive Theorie ausdehnen lassen:

Für $s=2$ lassen sich die Abschätzungen für $T^{-}$wie zuvor durchführen; man erhält

$$
\left\|T^{-}\right\|_{1} \leq c \cdot E^{-\frac{1}{2}} \cdot(E r)^{\alpha} \quad \text { für } 0<\alpha<\frac{3}{2} .
$$

Die Abschätzungen für $T^{+}$sind schwieriger zu handhaben. Um in (7.2.111) das Integral noch abschätzen zu können, muß man dort (zumindest infinitesimal) negative Werte für $\alpha$ zulassen. Dies ist im Fall $m>0$ auch möglich, denn man kann den Operator $A=$ $Q(E) \omega^{\alpha}\left(1+r^{2} \omega^{2}\right)^{2 s}$ dann in der Norm abschätzen durch

$$
\|A\|=\left\|Q(E) \frac{\left(1+r^{2} \omega^{2}\right)^{2 s}}{\left(\vec{p}^{2}+m^{2}\right)^{-|\alpha| / 2}}\right\| \leq m^{\alpha} \cdot \text { const. } \quad(\alpha<0) .
$$

Die Operatoren $C$ und $D$ bleiben unverändert - sie waren unabhängig von $\alpha$ definiert. Man erhält auf diese Weise für $T^{+}$Abschätzungen der Form

$$
\left\|T^{+}\right\|_{1} \leq c \cdot E^{-\frac{1}{2}} \cdot\left(E r^{\beta}\right)^{\frac{1}{2}},
$$

wobei die Konstante $c$ von $m$ abhängt und $0<\beta<1$ beliebig vorgegeben werden kann.

Dies reicht nicht aus, um Eigenschaft 7.2 und damit das asymptotische Phasenraumkriterium zu etablieren. Die Abschätzung kann aber dann interessant sein, wenn man das Phasenraumkriterium in eine nicht-skaleninvariante Form abändern will; siehe dazu die Diskussion in Abschnitt 8.17.

\subsection{Die kleinste obere Schranke zweier Operatoren}

Es seien $A, B \in \mathfrak{B}(\mathcal{H})$ zwei positive Operatoren. Wir werden im folgenden einen zuerst in [B.187] beschriebenen Operator $C$ konstruieren, der in gewisser Weise die kleinste obere Schranke von $A$ und $B$ darstellt: $C$ soll der kleinste Operator sein, der die Gleichung

$$
C^{n} \geq A^{n}, \quad C^{n} \geq B^{n}
$$


für alle natürlichen Zahlen $n$ erfüllt, wobei sich „klein“ auf die übliche Ordnungsstruktur selbstadjungierter Operatoren bezieht.

Für einen solchen Operator muß offenbar

$$
C \geq\left(\frac{1}{2} A^{n}+\frac{1}{2} B^{n}\right)^{\frac{1}{n}} \quad \forall n \in \mathbb{N}
$$

gelten. Wir versuchen, $C$ als den Grenzwert einer Teilfolge der rechten Seite zu definieren, und setzen dazu

$$
C_{m}:=\left(\frac{1}{2}\left(A^{2^{m}}+B^{2^{m}}\right)\right)^{2^{-m}}
$$

Lemma 7.12. Die Folge $C_{m}$ ist monoton wachsend und gleichmäßig in der Operatornorm beschränkt.

Beweis. Allgemein gilt für selbstadjungierte Operatoren $D, E \in \mathfrak{B}(\mathcal{H})$ :

$$
\frac{1}{2} D^{2}+\frac{1}{2} E^{2}=\left(\frac{1}{2} D+\frac{1}{2} E\right)^{2}+\underbrace{\left(\frac{1}{2} D-\frac{1}{2} E\right)^{2}}_{\geq 0} \geq\left(\frac{1}{2} D+\frac{1}{2} E\right)^{2} .
$$

Da die Funktion $x \mapsto x^{p}(p \leq 1)$ operator-monoton ist, folgt speziell mit $D=A^{2^{m}}$, $E=B^{2^{m}}$ :

$$
\left(\frac{1}{2} A^{2^{m+1}}+\frac{1}{2} B^{2^{m+1}}\right)^{2^{-m-1}} \geq\left(\frac{1}{2} A^{2^{m}}+\frac{1}{2} B^{2^{m}}\right)^{2^{-m}} \Rightarrow C_{m+1} \geq C_{m} .
$$

Weiterhin hat man für die Norm der $C_{m}$

$$
\left\|C_{m}\right\| \leq\left(\frac{1}{2}\|A\|^{2^{m}}+\frac{1}{2}\|B\|^{2^{m}}\right)^{2^{-m}} \leq \max \{\|A\|,\|B\|\}
$$

(man betrachte die Ungleichung zunächst mit $2^{m}$ exponentiert); damit ist eine gleichmäßige Normschranke gefunden.

Aus dem eben bewiesenen Lemma folgt gerade, daß $C_{m}$ stark gegen einen Operator $C \in \mathfrak{B}(\mathcal{H})$ konvergiert:

$$
C:=\operatorname{s-lim}_{m \rightarrow \infty} C_{m}
$$

$C$ ist offenbar positiv, da die $C_{m}$ es sind. Außerdem ist Relation (7.2.120) erfüllt, denn

$$
C^{n}=\operatorname{s-lim}_{m \rightarrow \infty}\left(\frac{1}{2} A^{2^{m}}+\frac{1}{2} B^{2^{m}}\right)^{n 2^{-m}} \geq \operatorname{s-lim}_{m \rightarrow \infty}\left(\frac{1}{2} A^{2^{m}}+0\right)^{n 2^{-m}}=A^{n}
$$

(der Exponent ist kleiner als 1 für große $m$ ); analoges gilt für $B$. Ist $D$ ein weiterer Operator, der (7.2.120) erfüllt, dann folgt wegen (7.2.121):

$$
D \geq C_{m} \forall m \in \mathbb{N} \quad \Rightarrow \quad D \geq C .
$$

Wir fassen die gewonnenen Ergebnisse nun zusammen: 
Satz 7.13. Seien $A, B \in \mathfrak{B}(\mathcal{H})$ positive Operatoren. Dann ist der Operator

$$
C=s-\lim \left(\frac{1}{2} A^{2^{m}}+\frac{1}{2} B^{2^{m}}\right)^{2^{-m}}
$$

ebenfalls positiv, und er erfüllt

$$
C^{n} \geq A^{n}, \quad C^{n} \geq B^{n} \quad \forall n \in \mathbb{N} .
$$

$C$ ist der kleinste Operator mit dieser Eigenschaft; er heißt kleinste obere Schranke von $A$ und B. Für seine Norm gilt

$$
\|C\| \leq \max \{\|A\|,\|B\|\}
$$

Vertauscht $D \in \mathfrak{B}(\mathcal{H})$ mit $A$ und $B$, so auch mit allen $C_{m}$ und deshalb mit $C$. Dabei kommt es nicht auf die $\mathbb{C}$-Linearität von $D$ an, wir können z.B. auch antilineare Operatoren zulassen. Es gilt also das

Korollar 7.14. Sei $D$ ein $\mathbb{R}$-linearer, beschränkter Operator mit $[D, A]=[D, B]=0$. Dann ist auch $[D, C]=0$.

Wir nehmen nun an, daß $A$ und $B$ in der Spurklasse liegen. Aus [Kos84] weiß man, daß für beliebige positive Operatoren $D, E \in \mathfrak{B}(\mathcal{H})$ und $0<c \leq 1$ gilt

$$
\left\|(D+E)^{c}\right\|_{1} \leq\left\|D^{c}\right\|_{1}+\left\|E^{c}\right\|_{1} .
$$

Daraus folgt für $m \in \mathbb{N}$ :

$$
\left\|C_{m}\right\|_{1}=\left(\frac{1}{2}\right)^{2^{-m}}\left\|\left(A^{2^{m}}+B^{2^{m}}\right)^{2^{-m}}\right\|_{1} \stackrel{(\overline{7.2 .129})}{\leq}\|A\|_{1}+\|B\|_{1} .
$$

Die Spurnormen $\left\|C_{m}\right\|_{1}$ sind also gleichmäßig nach oben beschränkt. Sei nun $\left\{e_{j}\right\}$ eine Orthonormalbasis von $\mathcal{H}$ und $N \in \mathbb{N}$ fest; da die $C_{m}$ positiv sind, folgt

$$
\sum_{j=1}^{N}\left(e_{j} \mid C_{m} e_{j}\right) \leq\|A\|_{1}+\|B\|_{1},
$$

für $m \rightarrow \infty$ also

$$
\sum_{j=1}^{N}\left(e_{j} \mid C e_{j}\right) \leq\|A\|_{1}+\|B\|_{1} .
$$

Die linke Seite konvergiert folglich für $N \rightarrow \infty$, woraus wir schließen, daß auch $C$ Spuroperator ist:

Lemma 7.15. Seien $A, B$ zwei positive Spurklasseoperatoren und $C$ ihre kleinste obere Schranke. Dann ist $C$ ebenfalls in der Spurklasse, und es gilt

$$
\|C\|_{1} \leq\|A\|_{1}+\|B\|_{1} .
$$




\subsection{Bestimmung des Feldinhalts}

Nachdem wir nun das asymptotische Phasenraumkriterium in den betrachteten Modellen etabliert haben, bleibt noch die Frage nach dem Feldinhalt der Theorie. Wie wir im Verlauf der Konstruktion in Kapitel 3 gesehen hatten, ist dieser nicht sofort aus den konstruierten approximierenden Abbildungen

$$
\psi=\sum_{j=1}^{N} \phi_{j} \sigma_{j}
$$

abzulesen. Zwar ist deren Rechts-Bild (also die lineare Hülle der $\phi_{j}$ für beliebig große $j$ ) eine Obermenge des Feldinhalts, diese Inklusion kann aber i. allg. echt sein. Genauer tragen nur die „primären Anteile“ der approximierenden Abbildung zum Punktfeldinhalt bei (vgl. Abschnitt 3.1).

Wir wollen jedoch nachweisen, daß in den betrachteten Modellen der freien Feldtheorie die lineare Hülle der $\phi_{j}$ mit dem Fredenhagen-Hertel-Feldinhalt $\Phi_{\mathrm{FH}}$ übereinstimmt. Dazu würde es nach Abschnitt 3.1 ausreichen zu zeigen, daß "sekundäre Anteile“ von Bild ${ }_{\mathrm{L}} \psi=$ Span $\left\{\sigma_{1}, \ldots, \sigma_{N}\right\}$ nicht vorhanden sind, d. h. daß

$$
\gamma(\sigma)<\Theta(N+1) \quad \forall \sigma \in \operatorname{Span}\left\{\sigma_{1}, \ldots, \sigma_{N}\right\}
$$

zumindest für gewisse $N$.

Es erweist sich jedoch als schwierig, für die Linearkombinationen der konkret konstruierten Funktionale $\sigma_{j}$ untere Abschätzungen anzugeben. Wir wählen daher einen etwas anderen, in gewisser Weise allgemeineren Ansatz: $\mathrm{Zu}$ einer beliebigen Rang- $N$-Abbildung $\psi_{N} \in \bar{\Psi}_{0}$ zeigen wir, daß sie $\Xi$ nicht besser approximieren kann als eine bestimmte, von $N$ abhängige untere Schranke. (Wir vermeiden auf diese Weise eine explizite Referenzierung der konstruierten Funktionale.) Dies erlaubt uns, die Dimension der Punktfeldräume $\Phi_{\bar{\gamma}}$ zu gegebenem $\bar{\gamma}$ von unten abzuschätzen. Da diese Schranken zumindest für gewisse $N$ mit dem in Abschnitt 7.2 berechneten Rang der approximierenden Abbildungen übereinstimmen, haben wir somit den Feldinhalt vollständig bestimmt.

\subsubsection{Vorgehensweise}

Wir betrachten eine beliebig vorgegebene Abbildung $\psi_{N} \in \bar{\Psi}_{0}$ vom Rang $N$ und interessieren uns für untere Abschätzungen des Ausdrucks

$$
\begin{aligned}
\left\|\Xi-\psi_{N}\right\|_{E, r} & =\sup _{\tau, A}\left\|\Xi(\tau, A)-\psi_{N}(\tau, A)\right\|_{E, r} \\
& =\sup _{\tau, A}\left\|\tau(A)-\psi_{N}(\tau, A)\right\|_{E, r} \quad\left(\tau \in \Sigma(E)_{1}, A \in \mathfrak{A}(r)_{1}\right) .
\end{aligned}
$$

Können wir hier zu jedem $E$ und $r$ ein $\tau \in \Sigma(E)$ und ein $A \in \mathfrak{A}(r)$ angeben (beide normiert), speziell etwa $A \in \operatorname{Kern}_{\mathrm{R}} \psi_{N}$, so daß

$$
|\tau(A)| \geq c \cdot(E r)^{\bar{\gamma}}
$$


mit einer von $E$ und $r$ unabhängigen Konstanten $c$, dann haben wir als untere Schranke für die "Approximationsgüte“ von $\psi_{N}$ erhalten:

$$
\left\|\Xi-\psi_{N}\right\|_{E, r} \geq c \cdot(E r)^{\bar{\gamma}}
$$

Wir können dann $E, r$ speziell so wählen, daß wir $\operatorname{dim} \Phi_{\bar{\gamma}+\epsilon}>N$ erhalten - siehe dazu die Überlegungen in Abschnitt 7.3.4.

Die Auswahl von $\tau$ und $A$ in (7.3.4) treffen wir aufgrund folgender Überlegung: Der Rechts-Kern von $\psi_{N}$ hat in $\mathfrak{A}(r)$ die Kodimension $N$. Sind nun $f_{0} \ldots f_{N} \in \mathcal{L}(r)$ fest gewählt und paarweise verschieden, dann gibt es also $a_{0} \ldots a_{N} \in \mathbb{C}$ (nicht alle 0 ), so daß

$$
A:=a_{0} W\left(f_{0}\right)+\ldots+a_{N} W\left(f_{N}\right) \in \operatorname{Kern}_{\mathrm{R}} \psi .
$$

Ohne Einschränkung der Allgemeinheit sei $A$ normiert. Man hat dann

$$
1=\|A\|=\left\|\sum_{i=0}^{N} a_{i} W\left(f_{i}\right)\right\| \leq \sum_{i=0}^{N}\left|a_{i}\right|\left\|W\left(f_{i}\right)\right\|=\sum_{i=0}^{N}\left|a_{i}\right| .
$$

Unser Ziel ist es, Zustände $\tau_{j} \in \Sigma(E)(j=0 \ldots N)$ zu finden, so daß

$$
\left|\tau_{j}(A)\right| \geq \text { const. } \cdot\left|\sum_{i} C_{i j} a_{i}\right|(E r)^{\bar{\gamma}}
$$

Dabei sind die $C_{i j}$ Konstanten. Wir wollen dann zeigen, daß diese Abschätzungen in einem gewissen Sinne unabhängig von den (in unserem Zugang unbestimmten) $a_{i}$ sind.円 Dazu ist folgende Aussage nützlich:

Lemma 7.16. Sei $C=\left(C_{i j}\right)$ eine invertierbare $n \times n$-Matrix. Dann gibt es $d>0$, so daß aus

$$
a \in \mathbb{C}^{n}, b_{j}>0,\left|\sum_{i=1}^{n} C_{i j} a_{i}\right|<b_{j} \quad \forall j \in\{1 \ldots n\}
$$

stets folgt

$$
\max _{j}\left|a_{j}\right|<d \cdot \max _{j} b_{j}
$$

Beweis. Seien $a, b_{j}$ wie oben; man berechnet

$$
\begin{aligned}
\max _{j} b_{j}^{2}>\max _{j}\left|\sum_{i} C_{i j} a_{i}\right|^{2} & \geq \frac{1}{n} \sum_{j}\left|\sum_{i} C_{i j} a_{i}\right|^{2}=\frac{1}{n}\|C a\|^{2} \\
& \geq \frac{1}{n}\left(\frac{1}{\left\|C^{-1}\right\|}\|a\|\right)^{2} \geq \underbrace{\frac{1}{n\left\|C^{-1}\right\|^{2}}}_{=: d^{2}} \max _{j}\left|a_{j}\right|^{2} .
\end{aligned}
$$

11 Das Auftreten der $a_{i}$ in den Abschätzungen (7.3.8) ist in gewissem Sinne der „generische Fall“, da die $\tau_{j}$ immer linear sind. 
Wir nehmen die Matrix $\left(C_{i j}\right)$ in (7.3.8) nun als invertierbar an; nach Multiplikation mit $(E r)^{-\bar{\gamma}}$ können wir das Lemma anwenden. Da wegen $(7.3 .7)$ hier $\max _{j}\left|a_{j}\right| \geq \frac{1}{N+1}$ gilt, erhalten wir ein $j$ mit

$$
\left|\tau_{j}(A)\right| \geq(E r)^{\bar{\gamma}} \cdot \text { const. }
$$

Unter den genannten Voraussetzungen an $C_{i j}$ haben wir damit untere Abschätzungen der Form (7.3.5) etabliert.

Es müssen nun die speziellen Funktionen[ $f_{i}$ und Funktionale $\tau_{j}$ konstruiert werden, wobei die letzteren wie bei der Etablierung des Phasenraumkriteriums auf Matrixelemente mit Einteilchen-Wellenfunktionen $g_{j}$ zurückgeführt werden. Wir beginnen daher wieder mit der Konstruktion gewisser Funktionen im Einteilchenraum.

\subsubsection{Funktionen im Einteilchenraum}

Unser Vorgehen ist in mancher Hinsicht sehr ähnlich (aber nicht identisch) zu dem in Abschnitt 7.2.2. Wir fixieren eine Testfunktion $f \in \mathcal{S}(\mathbb{R})$ mit $f \geq 0, f \not \equiv 0$ und $f(x)=0$ für $|\vec{x}|>1$; dann setzen wir für $r>0$ :

$$
f_{r}(\vec{x}):=f\left(r^{-1}|\vec{x}|\right) .
$$

$f_{r}$ ist dann in der Kugel vom Radius $r$ um 0 lokalisiert; damit ist $f_{r}$ ein Kandidat für die Konstruktion der Weyloperatoren aus $\mathfrak{A}(r)$.

Weiterhin benötigen wir energiebeschränkte Funktionen im Impulsraum. Dazu sei für $E>m$

$$
g_{E}(\vec{p})=\left\{\begin{array}{ll}
1 & \text { für } \omega(\vec{p}) \leq E, \\
0 & \text { sonst; }
\end{array} \quad g_{E} \in Q(E) \mathcal{K} .\right.
$$

Wir betrachten im folgenden für $s$-stellige Multiindizes ${ }^{[3]} \kappa, \lambda$ die Funktionen

$$
\omega^{ \pm \frac{1}{2}} p^{\kappa} g_{E} \quad \text { und } \quad \omega^{\mp \frac{1}{2}} p^{\lambda} \tilde{f}_{r} .
$$

Man beachte, daß dabei die Energiebeschränktheit erhalten bleibt; außerdem stört der Faktor $p^{\lambda}$ die Lokalisierung des Trägers nicht, da er sich als Ableitung $-i \partial_{\lambda}$ in den Ortsraum „überwälzen“ läßt. Die Funktionen sind linear unabhängig. Wir berechnen nun diverse Skalarprodukte zwischen ihnen, zunächst diejenigen zwischen den $f$ 's:

$$
\begin{aligned}
& \left\langle\omega^{\mp \frac{1}{2}} p^{\kappa} \tilde{f}_{r} \mid \omega^{\mp \frac{1}{2}} p^{\lambda} \tilde{f}_{r}\right\rangle \\
& \quad=\int d^{s} p{\sqrt{\vec{p}^{2}+m^{2}}}^{\mp 1} p^{\kappa+\lambda}(2 \pi)^{-s} \int d^{s} x d^{s} y \overline{f\left(r^{-1}|\vec{x}|\right)} f\left(r^{-1}|\vec{y}|\right) e^{i \vec{p}(\vec{x}-\vec{y})} \\
& \stackrel{\text { (Skalierung) }}{=} \frac{r^{-|\lambda|-|\kappa|+s \pm 1}}{(2 \pi)^{s}} \int d^{s} p \sqrt{\vec{p}^{2}+(r m)^{2}}{ }^{\mp 1} p^{\kappa+\lambda} \int d^{s} x d^{s} y \overline{f(\vec{x})} f(\vec{y}) e^{i \vec{p}(\vec{x}-\vec{y})} .
\end{aligned}
$$

\footnotetext{
${ }^{12}$ Wir werden sie später mit $f_{i}^{*}$ bezeichnen.

13 Zur Multiindex-Schreibweise siehe wiederum Anhang 7.2.A.
} 
Wir betrachten diesen Ausdruck im Limes $r m \rightarrow 0$. Der Term $\left(\vec{p}^{2}+(r m)^{2}\right)^{\mp \frac{1}{2}}$ kann gleichmäßig in $r m$ abgeschätzt werden (es ergibt sich höchstens eine Singularität wie $|\vec{p}|^{-1}$, die für $s \geq 2$ integrierbar bleibt); der vom Integral über $x$ und $y$ herrührende Term fällt mit $|\vec{p}|$ sehr schnell ab ( $f$ ist Testfunktion). Nach dem Satz von der majorisierten Konvergenz kann man den Limes also unter dem Integral ausführen und erhält

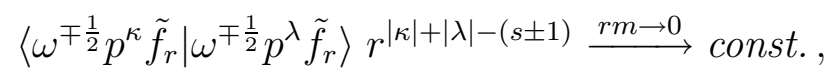

wobei die Konstante nur noch von $\kappa$ und $\lambda$ abhängt; sie verschwindet nicht, falls $\kappa=\lambda$.

Das Skalarprodukt zwischen den $g$ 's ergibt sich so:

$$
\begin{aligned}
& \left\langle\omega^{ \pm \frac{1}{2}} p^{\kappa} g_{E} \mid \omega^{ \pm \frac{1}{2}} p^{\lambda} g_{E}\right\rangle=\int_{\omega \leq E} d^{s} p{\sqrt{\vec{p}^{2}+m^{2}}}^{ \pm 1} p^{\kappa+\lambda} \\
& =\int_{0}^{\sqrt{E^{2}-m^{2}}} d|\vec{p}| \sqrt{|\vec{p}|^{2}+m^{2}} \pm 1|\vec{p}|^{|\kappa|+|\lambda|+s-1} \underbrace{\int d \Omega(p) \frac{p^{\kappa+\lambda}}{|\vec{p}||\kappa|+|\lambda|}}_{=: \Delta(\kappa+\lambda)} \\
& \stackrel{\text { (Skalierung) }}{=} E^{|\kappa|+|\lambda|+s \pm 1} \Delta(\kappa+\lambda) \int_{0}^{\sqrt{1-m^{2} / E^{2}}} d q \sqrt{q^{2}+\left(\frac{m}{E}\right)^{2}} q^{|\kappa|+|\lambda|+s-1} \text {. }
\end{aligned}
$$

Der oben definierte Ausdruck $\Delta(\kappa+\lambda)$ wird in Anhang 7.3.A näher untersucht. Wieder läßt sich im Limes $\frac{E}{m} \rightarrow \infty$ der Satz von der majorisierten Konvergenz anwenden, womit sich ergibt, daß

$$
\left(\omega^{ \pm \frac{1}{2}} p^{\kappa} g_{E} \mid \omega^{ \pm \frac{1}{2}} p^{\lambda} g_{E}\right) E^{-|\kappa|-|\lambda|-(s \pm 1)} \stackrel{\frac{E}{m} \rightarrow \infty}{\longrightarrow} \text { const }
$$

Auch diese Konstante ist nicht 0 , falls $\kappa=\lambda$.

Nun berechnen wir das Skalarprodukt zwischen einem $f$ und einem $g$ :

$$
\begin{aligned}
& \left\langle\omega^{ \pm \frac{1}{2}} p^{\kappa} g_{E} \mid \omega^{\mp \frac{1}{2}} p^{\lambda} \tilde{f}_{r}\right\rangle=\left\langle p^{\kappa} g_{E} \mid p^{\lambda} \tilde{f}_{r}\right\rangle=(2 \pi)^{-\frac{s}{2}} \int_{\omega \leq E} d^{s} p p^{\kappa+\lambda} \int_{|x| \leq r} d^{s} x e^{-i \vec{p} \vec{x}} f\left(r^{-1}|\vec{x}|\right) \\
& \stackrel{\text { (Skalierung) }}{=} r^{-|\kappa|-|\lambda|} \int_{\vec{p}^{2} \leq\left(E^{2}-m^{2}\right) r^{2}} d^{s} p p^{\kappa+\lambda} \underbrace{(2 \pi)^{-\frac{s}{2}} \int_{|x| \leq 1} d^{s} x e^{-i \vec{p} \vec{x}} f(|\vec{x}|)}_{=\tilde{f}(\vec{p})} \\
& \text { ( } \tilde{f}(\vec{p}) \text { hängt nur von }|\vec{p}| \text { ab.) } \\
& \stackrel{\text { (Skalierung) }}{=} r^{-|\kappa|-|\lambda|}(E r)^{s+|\kappa|+|\lambda|} \int_{\vec{p}^{2} \leq 1-m^{2} / E^{2}} d|\vec{p}||\vec{p}|^{|\kappa|+|\lambda|+s-1} \tilde{f}(|\vec{p}| \cdot E r) \int d \Omega(p) \frac{p^{\kappa+\lambda}}{|\vec{p}||\kappa|+|\lambda|} \\
& =r^{s} E^{s+|\kappa|+|\lambda|} \Delta(\kappa+\lambda) \int_{0}^{\sqrt{1-m^{2} / E^{2}}} q^{|\kappa|+|\lambda|+s-1} \tilde{f}(q \cdot E r) d q .
\end{aligned}
$$


Im Limes $\operatorname{Er} \rightarrow 0, \frac{E}{m} \rightarrow \infty$ kann noch einmal der Satz von der majorisierten Konvergenz angewandt werden; Ausführen der Integration ergibt dann

$$
\left\langle\omega^{ \pm \frac{1}{2}} p^{\kappa} g_{E} \mid \omega^{\mp \frac{1}{2}} p^{\lambda} \tilde{f}_{r}\right\rangle r^{-s} E^{-s-|\kappa|-|\lambda|} \stackrel{E r \rightarrow 0}{\longrightarrow} \frac{\Delta(\kappa+\lambda)}{s+|\kappa|+|\lambda|} \tilde{f}(0) .
$$

Die Funktion $f$ war so gewählt, daß $\tilde{f}(0) \neq 0$.

Wir normieren nun die oben betrachteten Funktionen:

$$
f_{\kappa}^{ \pm}:=\frac{i^{|\kappa|} \omega^{\mp \frac{1}{2}} p^{\kappa} \tilde{f}_{r}}{\left\|\omega^{\mp \frac{1}{2}} p^{\kappa} \tilde{f}_{r}\right\|} \in \mathcal{L}^{ \pm}(r) \quad ; \quad g_{\kappa}^{ \pm}:=\frac{i^{|\kappa|} \omega^{ \pm \frac{1}{2}} p^{\kappa} g_{E}}{\left\|\omega^{ \pm \frac{1}{2}} p^{\kappa} g_{E}\right\|} \in Q(E) \mathcal{K} .
$$

Die $f_{\kappa}^{ \pm}$sind dann linear unabhängig und invariant unter $J$, denn man hat $[J, \omega]=0$ und $J p_{k}=-p_{k} J$. Entsprechendes gilt für die $g_{\kappa}^{ \pm}$. Unter Verwendung der Ergebnisse (7.3.15) und (7.3.17) erhält man:"[4

$$
\begin{array}{lll}
\left\langle g_{\kappa}^{ \pm} \mid g_{\lambda}^{ \pm}\right\rangle & \underset{\frac{E r \rightarrow 0}{m} \rightarrow \infty}{\stackrel{E}{m}} \text { const. }(\kappa, \lambda) ; \\
\left\langle f_{\kappa}^{ \pm} \mid f_{\lambda}^{ \pm}\right\rangle & \underset{\frac{E r \rightarrow 0}{m} \rightarrow \infty}{\stackrel{E}{\longrightarrow}} \text { const.' }(\kappa, \lambda) .
\end{array}
$$

Die Konstanten const. $(\kappa, \lambda)$ und const.' $(\kappa, \lambda)$ sind gleich 1 , falls $\kappa=\lambda$. Für die gemischten Skalarprodukte hat man

$$
\left\langle f_{\kappa}^{ \pm} \mid g_{\lambda}^{ \pm}\right\rangle(E r)^{-\vartheta^{ \pm}(\kappa)} \underset{\frac{E}{m} \rightarrow \infty}{\stackrel{E r \rightarrow 0}{\longrightarrow}} \quad b_{1}^{ \pm}(\kappa) \frac{\Delta(\kappa+\lambda)}{s+|\kappa|+|\lambda|} b_{2}^{ \pm}(\lambda) ;
$$

dabei ist

$$
\vartheta^{ \pm}(\kappa):=|\kappa|+\frac{s \mp 1}{2},
$$

die $b_{1}^{ \pm}(\kappa), b_{2}^{ \pm}(\lambda)$ sind wiederum nichtverschwindende Konstanten. Läßt man $\kappa$ und $\lambda$ über eine endliche Menge $M$ von Multiindizes laufen, dann ist die Matrix

$$
\frac{\Delta(\kappa+\lambda)}{s+|\kappa|+|\lambda|} \quad(\kappa, \lambda \in M)
$$

nach Lemma 7.20 in Anhang 7.3.A invertierbar; die Multiplikation mit den $b_{1}^{ \pm}(\kappa), b_{2}^{ \pm}(\lambda)$ kann als Multiplikation mit zwei Diagonalmatrizen aufgefaßt werden, die ihrerseits invertierbar sind. Die rechte Seite von (7.3.23) bildet somit eine nichtsinguläre Matrix $B_{\kappa \lambda}^{ \pm}$.

Wir können auf die Details der Funktionen $f_{\kappa}^{ \pm}, g_{\lambda}^{ \pm}$jetzt verzichten und numerieren sie daher mit natürlichen Zahlen $j$ durch, wobei wir die Reihenfolge so wählen, daß das resultierende $\vartheta^{ \pm}(j)$ monoton mit $j$ wächst. Die im folgenden relevanten Ergebnisse fassen wir noch einmal zusammen bzw. formulieren sie für beliebigen Einteilchenraum als Forderung:

\footnotetext{
${ }^{14}$ Man beachte, daß $E r \rightarrow 0, \frac{E}{m} \rightarrow \infty$ auch $r m \rightarrow 0$ impliziert.
} 
Eigenschaft 7.17. $Z u E \geq E_{0}, r \leq r_{0}, E r \leq 1$ gibt es J-invariante Vektoren

$$
f_{j}^{ \pm} \in \mathcal{L}^{ \pm}(r), \quad g_{j}^{ \pm} \in Q(E) \mathcal{K} \quad(j \in \mathbb{N}),
$$

die in $\mathcal{L}^{ \pm}(r)$ bzw. $Q(E) \mathcal{K}$ linear unabhängig sind. Weiterhin existieren zwei monoton wachsende Funktionen $\vartheta^{ \pm}: \mathbb{N} \rightarrow \mathbb{R}^{+}$mit $\vartheta^{ \pm}(j) \rightarrow \infty$ für $j \rightarrow \infty$, so daß

$$
\begin{array}{rll}
\left\langle f_{j}^{ \pm} \mid f_{k}^{ \pm}\right\rangle & \stackrel{E r \rightarrow 0}{\underset{E \rightarrow \infty}{\longrightarrow}} c_{f}^{ \pm}(j, k), \\
\left\langle g_{j}^{ \pm} \mid g_{k}^{ \pm}\right\rangle & \stackrel{E r \rightarrow 0}{\underset{E \rightarrow \infty}{\longrightarrow}} c_{g}^{ \pm}(j, k), \\
\left\langle g_{j}^{ \pm} \mid f_{k}^{ \pm}\right\rangle(E r)^{-\vartheta^{ \pm}(k)} & \underset{E \rightarrow \rightarrow 0}{\stackrel{E r \rightarrow 0}{\longrightarrow}} & B_{j k}^{ \pm} .
\end{array}
$$

Die Konstanten $c_{f}^{ \pm}(j, k)$ und $c_{g}^{ \pm}(j, k)$ verschwinden nicht, falls $j=k$; die Matrix $B_{j k}^{ \pm}(j, k \leq$ $N)$ ist für jedes $N \in \mathbb{N}$ invertierbar. $E_{0}$ und $r_{0}$ sind positive Konstanten.

Wie interessieren uns besonders für den Fall, in dem die $\vartheta^{ \pm}$mit den in Eigenschaft 7.1 erwähnten Funktionen übereinstimmen, wie dies beim reellen skalaren Feld der Fall ist; unsere Überlegungen sind aber unabhängig davon. Die Tatsache der Invertierbarkeit der Matrizen $B_{j k}^{ \pm}$soll noch etwas umformuliert werden, bevor wir sie im folgenden verwenden:

Lemma 7.18. Sei $N \in \mathbb{N}$ und

$$
g=\sum_{j=1}^{N} c_{j}(E, r) g_{j}^{ \pm}, \quad \text { wobei } \quad c_{j}(E, r) \underset{E \rightarrow \infty}{\stackrel{E r \rightarrow 0}{\longrightarrow}} c_{j}^{0}
$$

mit Konstanten $c_{j}^{0}$, die nicht alle verschwinden. Dann gilt für $1 \leq k \leq N$

$$
\left\langle g \mid f_{k}^{ \pm}\right\rangle(E r)^{-\vartheta^{ \pm}(k)} \quad \underset{E \rightarrow \infty}{\stackrel{E r \rightarrow 0}{\longrightarrow}} d_{k}^{0}
$$

mit Konstanten $d_{k}^{0}$, die ebenfalls nicht alle verschwinden.

Beweis. Man hat nach Eigenschaft 7.17:

$$
\left\langle g \mid f_{k}^{ \pm}\right\rangle(E r)^{-\vartheta^{ \pm}(k)}=\sum_{j=1}^{N} \overline{c_{j}(E, r)}\left\langle g_{j}^{ \pm} \mid f_{k}^{ \pm}\right\rangle(E r)^{-\vartheta^{ \pm}(k)} \quad \underset{E \rightarrow \infty}{\stackrel{E r \rightarrow 0}{\longrightarrow}} \sum_{j=1}^{N} \overline{c_{j}^{0}} B_{j k}^{ \pm}=: d_{k}^{0}
$$

Wegen der Invertierbarkeit von $B_{j k}^{ \pm}$können nicht alle $d_{k}^{0}$ verschwinden.

\subsubsection{Verallgemeinertes Gram-Schmidt-Verfahren}

Das Verhalten der Skalarprodukte $\left\langle f_{j}^{ \pm} \mid g_{k}^{ \pm}\right\rangle$für große $E$ und kleine $E r$ ist nach Eigenschaft 7.17 bekannt. Für das folgende erweist es sich aber als günstig, über noch einfachere Relationen für diese Ausdrücke zu verfügen, etwa $\left\langle f_{j}^{ \pm} \mid g_{k}^{ \pm}\right\rangle \propto \delta_{j k}$. Wir werden dies durch eine Variante des bekannten Gram-Schmidt'schen Orthogonalisierungsverfahrens erreichen. Die Vorzeichen \pm werden im folgenden fortgelassen, die Konstruktion verläuft in beiden Fällen völlig gleich. 
Wir konstruieren nun rekursiv Funktionen $\hat{f}_{n}, \hat{g}_{n}$, die folgende Eigenschaften erfüllen sollen:

$$
\begin{aligned}
& J \hat{f}_{n}=\hat{f}_{n} ; \quad J \hat{g}_{n}=\hat{g}_{n} \\
& \operatorname{Span}\left\{f_{1}, \ldots, f_{n}\right\}=\operatorname{Span}\left\{\hat{f}_{1}, \ldots, \hat{f}_{n}\right\} \\
& \operatorname{Span}\left\{g_{1}, \ldots, g_{n}\right\}=\operatorname{Span}\left\{\hat{g}_{1}, \ldots, \hat{g}_{n}\right\} \\
& \left\langle f_{k} \mid \hat{f}_{n}\right\rangle \quad \underset{E \rightarrow \infty}{\stackrel{E r \rightarrow 0}{\longrightarrow}} \text { const. }(k, n) \quad \forall k \in \mathbb{N} \\
& \left\langle\hat{f}_{k} \mid \hat{f}_{n}\right\rangle \quad \underset{E \rightarrow \infty}{\stackrel{E r \rightarrow 0}{\longrightarrow}} \text { const. }(k, n) \quad \forall k \leq n \\
& \left\langle g_{k} \mid \hat{g}_{n}\right\rangle \quad \underset{E \rightarrow \infty}{\stackrel{E r \rightarrow 0}{\longrightarrow}} \text { const. }(k, n) \quad \forall k \in \mathbb{N} \\
& \left\langle\hat{g}_{k} \mid \hat{g}_{n}\right\rangle \quad \underset{E \rightarrow \infty}{\stackrel{E r \rightarrow 0}{\longrightarrow}} \text { const. }(k, n) \quad \forall k \leq n \\
& \left\langle g_{k} \mid \hat{f}_{n}\right\rangle(E r)^{-\vartheta(n)} \quad \underset{E \rightarrow \infty}{\stackrel{E r \rightarrow 0}{\longrightarrow}} \text { const. }(k, n) \quad \forall k \in \mathbb{N} \\
& \left\langle\hat{g}_{n} \mid f_{k}\right\rangle(E r)^{-\vartheta(k)} \quad \underset{E \rightarrow \infty}{\stackrel{E r \rightarrow 0}{\longrightarrow}} \text { const. }(k, n) \quad \forall k \in \mathbb{N} \\
& \left\langle\hat{g}_{n} \mid \hat{f}_{k}\right\rangle=0 \quad \forall k<n \\
& \left\langle\hat{g}_{k} \mid \hat{f}_{n}\right\rangle=0 \quad \forall k<n \\
& \left\langle\hat{g}_{n} \mid \hat{f}_{n}\right\rangle(E r)^{-\vartheta(n)} \quad \underset{E \rightarrow \infty}{\stackrel{E r \rightarrow 0}{\longrightarrow}} \text { const. }(n) \neq 0
\end{aligned}
$$

Induktionsanfang $(n=1)$ : Wir setzen

$$
\hat{f}_{1}:=f_{1}, \quad \hat{g}_{1}:=g_{1} .
$$

(7.3.28) und (7.3.29) sind trivial erfüllt; (7.3.30) - (7.3.38) ergeben sich aus den entsprechenden Eigenschaften der $f_{j}$ und $g_{j}$.

Induktionsschritt $(n-1 \rightarrow n)$ : Wir definieren

$$
\begin{aligned}
& \hat{f}_{n}:=f_{n}-\sum_{j=1}^{n-1} \frac{\left\langle\hat{g}_{j} \mid f_{n}\right\rangle}{\left\langle\hat{g}_{j} \mid \hat{f}_{j}\right\rangle} \hat{f}_{j}, \\
& \hat{g}_{n}:=g_{n}-\sum_{j=1}^{n-1} \frac{\left\langle g_{n} \mid \hat{f}_{j}\right\rangle}{\left\langle\hat{g}_{j} \mid \hat{f}_{j}\right\rangle} \hat{g}_{j} .
\end{aligned}
$$

(Die Nenner sind nach Induktionsvoraussetzung (7.3.38) nicht 0 für genügend kleine $E r$ und große $E$.) Diese Funktionen sind wieder invariant unter $J$, denn die skalaren Faktoren sind reell. 
(7.3.28) ist erfüllt, denn einerseits gilt $\hat{f}_{n} \in \operatorname{Span}\left\{f_{1}, \ldots, f_{n}\right\}$ nach Definition, andererseits ist $\hat{f}_{n} \notin \operatorname{Span}\left\{f_{1}, \ldots, f_{n-1}\right\}$, weil die $f_{j}$ linear unabhängig sind; also wird der von den $\hat{f}$ erzeugte Unterraum echt größer. Ebenso folgt (7.3.29).

$\mathrm{Zu}(7.3 .30):$ Für $k \in \mathbb{N}$ gilt

$$
\begin{aligned}
& \left\langle f_{k} \mid \hat{f}_{n}\right\rangle=\left\langle f_{k} \mid f_{n}\right\rangle-\sum_{j=1}^{n-1} \frac{\left\langle\hat{g}_{j} \mid f_{n}\right\rangle}{\left\langle\hat{g}_{j} \mid \hat{f}_{j}\right\rangle}\left\langle f_{k} \mid \hat{f}_{j}\right\rangle \\
& =\left\langle f_{k} \mid f_{n}\right\rangle-\sum_{j=1}^{n-1} \frac{\left\langle\hat{g}_{j} \mid f_{n}\right\rangle(E r)^{-\vartheta(n)}}{\left\langle\hat{g}_{j} \mid \hat{f}_{j}\right\rangle(E r)^{-\vartheta(j)}}(E r)_{\vartheta(n)-\vartheta(j)}^{\geq 0}\left\langle f_{k} \mid \hat{f}_{j}\right\rangle \quad \underset{E \rightarrow \infty}{\stackrel{E r \rightarrow 0}{\longrightarrow}} \text { const. }
\end{aligned}
$$

nach Induktionsvoraussetzung (7.3.30), (7.3.35) und (7.3.38). Ebenso erhält man (7.3.32).

$\mathrm{Zu}(7.3 .31):$ Sei $k \leq n$, dann ist

$$
\begin{aligned}
\left\langle\hat{f}_{k} \mid \hat{f}_{n}\right\rangle=\left\langle f_{k} \mid f_{n}\right\rangle-\sum_{j=1}^{n-1} \frac{\left\langle\hat{g}_{j} \mid f_{n}\right\rangle}{\left\langle\hat{g}_{j} \mid \hat{f}_{j}\right\rangle}\left\langle\hat{f}_{j} \mid f_{k}\right\rangle-\sum_{j=1}^{k-1} \frac{\left\langle\hat{g}_{j} \mid f_{k}\right\rangle}{\left\langle\hat{g}_{j} \mid \hat{f}_{j}\right\rangle}\left\langle\hat{f}_{j} \mid f_{n}\right\rangle & \\
& +\sum_{j=1}^{n-1} \sum_{l=1}^{k-1} \frac{\left\langle\hat{g}_{j} \mid f_{n}\right\rangle}{\left\langle\hat{g}_{j} \mid \hat{f}_{j}\right\rangle} \frac{\left\langle\hat{g}_{l} \mid f_{k}\right\rangle}{\left\langle\hat{g}_{l} \mid \hat{f}_{l}\right\rangle}\left\langle\hat{f}_{j} \mid \hat{f}_{l}\right\rangle \quad \underset{E \rightarrow \infty}{\stackrel{E r \rightarrow 0}{\longrightarrow}} \text { const. }
\end{aligned}
$$

unter Verwendung von (7.3.30), (7.3.31), (7.3.35) und (7.3.38). Genauso ergibt sich (7.3.33).

$\mathrm{Zu}(7.3 .34):$ Für $k \in \mathbb{N}$ berechnet man

$$
\begin{aligned}
\left\langle g_{k} \mid \hat{f}_{n}\right\rangle(E r)^{-\vartheta(n)}=\left\langle g_{k}\right| & \left.f_{n}\right\rangle(E r)^{-\vartheta(n)} \\
& \quad-\sum_{j=1}^{n-1} \frac{\left\langle\hat{g}_{j} \mid f_{n}\right\rangle(E r)^{-\vartheta(n)}}{\left\langle\hat{g}_{j} \mid \hat{f}_{j}\right\rangle(E r)^{-\vartheta(j)}}\left\langle g_{k} \mid \hat{f}_{j}\right\rangle(E r)^{-\vartheta(j)} \quad \underset{E \rightarrow \infty}{\stackrel{E r \rightarrow 0}{\longrightarrow}} \text { const. }
\end{aligned}
$$

mit Voraussetzung (7.3.34), (7.3.35) und (7.3.38). Gleichung (7.3.35) folgt analog.

$\mathrm{Zu}(7.3 .37)$ : Es sei $k \leq n$; dann gilt identisch für alle $E$ und $r$ :

$$
\left\langle\hat{g}_{k} \mid \hat{f}_{n}\right\rangle=\left\langle\hat{g}_{k} \mid f_{n}\right\rangle-\sum_{j=1}^{n-1} \frac{\left\langle\hat{g}_{j} \mid f_{n}\right\rangle}{\left\langle\hat{g}_{j} \mid \hat{f}_{j}\right\rangle} \underbrace{\left\langle\hat{g}_{k} \mid \hat{f}_{j}\right\rangle}_{\propto \delta_{j k}}=\left\langle\hat{g}_{k} \mid f_{n}\right\rangle-\frac{\left\langle\hat{g}_{k} \mid f_{n}\right\rangle}{\left\langle\hat{g}_{k} \mid \hat{f}_{k}\right\rangle}\left\langle\hat{g}_{k} \mid \hat{f}_{k}\right\rangle=0 .
$$

Eine ähnliche Rechnung zeigt (7.3.36).

$\mathrm{Zu}(7.3 .38)$ : Man hat aufgrund von Voraussetzung (7.3.34) - (7.3.38)

$$
\begin{aligned}
& \left\langle\hat{g}_{n} \mid \hat{f}_{n}\right\rangle(E r)^{-\vartheta(n)}=\left\langle g_{n} \mid f_{n}\right\rangle(E r)^{-\vartheta(n)} \\
& -\sum_{j=1}^{n-1} \frac{\left\langle\hat{g}_{j} \mid f_{n}\right\rangle}{\left\langle\hat{g}_{j} \mid \hat{f}_{j}\right\rangle}\left\langle g_{n} \mid \hat{f}_{j}\right\rangle(E r)^{-\vartheta(n)}-\sum_{j=1}^{n-1} \frac{\left\langle g_{n} \mid \hat{f}_{j}\right\rangle}{\left\langle\hat{g}_{j} \mid \hat{f}_{j}\right\rangle}\left\langle\hat{g}_{j} \mid f_{n}\right\rangle(E r)^{-\vartheta(n)} \\
& +\sum_{j, k=1}^{n-1} \frac{\left\langle\hat{g}_{j} \mid f_{n}\right\rangle}{\left\langle\hat{g}_{j} \mid \hat{f}_{j}\right\rangle} \frac{\left\langle g_{n} \mid \hat{f}_{k}\right\rangle}{\left\langle\hat{g}_{k} \mid \hat{f}_{k}\right\rangle} \underbrace{\left\langle\hat{g}_{k} \mid \hat{f}_{j}\right\rangle}_{\propto \delta_{j k}}(E r)^{-\vartheta(n)}
\end{aligned}
$$

(Die drei Summen sind also gleich!)

$$
=\left\langle g_{n} \mid f_{n}\right\rangle(E r)^{-\vartheta(n)}-\sum_{j=1}^{n-1} \frac{\left\langle\hat{g}_{j} \mid f_{n}\right\rangle(E r)^{-\vartheta(n)}\left\langle g_{n} \mid \hat{f}_{j}\right\rangle(E r)^{-\vartheta(j)}}{\left\langle\hat{g}_{j} \mid \hat{f}_{j}\right\rangle(E r)^{-\vartheta(j)}} \quad \underset{E \rightarrow \infty}{\stackrel{E r \rightarrow 0}{\longrightarrow}} \text { const. }
$$


$\mathrm{Zu}$ zeigen ist noch, daß diese Konstante nicht verschwindet. Dazu nehmen wir an, daß $\left\langle\hat{g}_{n} \mid \hat{f}_{n}\right\rangle(E r)^{-\vartheta(n)} \rightarrow 0$. Wegen (7.3.36) gilt dann $\left\langle\hat{g}_{n} \mid \hat{f}_{k}\right\rangle(E r)^{-\vartheta(k)} \rightarrow 0 \forall k \leq n$, und aufgrund von $(\overline{7.3 .28})$ auch $\left\langle\hat{g}_{n} \mid f_{k}\right\rangle(E r)^{-\vartheta(k)} \rightarrow 0 \forall k \leq n$. Andererseits erfüllt

$$
\hat{g}_{n}=g_{n}-\sum_{j=1}^{n-1} \frac{\left\langle g_{n} \mid \hat{f}_{j}\right\rangle}{\left\langle\hat{g}_{j} \mid \hat{f}_{j}\right\rangle} \hat{g}_{j}
$$

die Voraussetzungen des Lemmas 7.18; daher gibt es ein $k$ mit $\left\langle\hat{g}_{n} \mid f_{k}\right\rangle(E r)^{-\vartheta(k)} \nrightarrow 0$. Damit ist ein Widerspruch erreicht.

Die Konstruktion der $\hat{f}_{n}$ und $\hat{g}_{n}$ ist nun abgeschlossen. Wir lassen das „Dach“ über den Symbolen wieder weg und verfügen damit über Funktionen $f_{n}^{ \pm}$und $g_{n}^{ \pm}$, deren Eigenschaften sich wie folgt zusammenfassen lassen:

$$
\begin{aligned}
& f_{n}^{ \pm} \in \mathcal{L}^{ \pm}(r) ; \quad g_{n}^{ \pm} \in Q(E) \mathcal{K} \\
& J f_{n}^{ \pm}=f_{n}^{ \pm} ; J g_{n}^{ \pm}=g_{n}^{ \pm} \\
& \left\langle f_{k}^{ \pm} \mid f_{n}^{ \pm}\right\rangle \quad \underset{E \rightarrow \infty}{\stackrel{E r \rightarrow 0}{\longrightarrow}} \text { const. }(k, n) ; \quad\left\langle g_{k}^{ \pm} \mid g_{n}^{ \pm}\right\rangle \quad \underset{E \rightarrow \infty}{\stackrel{E r \rightarrow 0}{\longrightarrow}} \text { const. }(k, n) \\
& \left\langle g_{k}^{ \pm} \mid f_{n}^{ \pm}\right\rangle=0 \quad \text { für } k \neq n \\
& \left\langle g_{n}^{ \pm} \mid f_{n}^{ \pm}\right\rangle(E r)^{-\vartheta^{ \pm}(n)} \quad \underset{E \rightarrow \infty}{\stackrel{E r \rightarrow 0}{\longrightarrow}} \text { const. }(n)
\end{aligned}
$$

Die Konstante in der letzten Gleichung verschwindet nicht.

\subsubsection{Zweite Quantisierung}

Nachdem wir die Situation im Einteilchenraum festgestellt und um einiges vereinfacht haben, bleiben noch die gewünschten energiebeschränkten Funktionale $\tau_{j}$ und die lokalen Weyloperatoren $W\left(f_{j}\right)$ zu konstruieren.

Wir beginnen auf der Seite der Funktionale: Mit Hilfe von Lemma 7.8 in Anhang 7.2.B verschaffen wir uns zu Multiindizes $\mu^{+}, \mu^{-}$beliebiger Stellenzahl ein Funktional $\tau_{\mu^{+} \mu^{-}}$, das auf Weyloperatoren $W(f)$ die Werte

$$
\tau_{\mu^{+} \mu^{-}}(W(f))=e^{-\frac{1}{2}\|f\|^{2}} \prod_{j}\left\langle f^{+} \mid g_{j}^{+}\right\rangle^{\mu_{j}^{+}} \prod_{k}\left\langle f^{-} \mid g_{k}^{-}\right\rangle^{\mu_{k}^{-}}
$$

annimmt. Da die $g_{j}^{ \pm}$energiebeschränkt sind, liefert Korollar 7.10, daß $\tau_{\mu^{+} \mu^{-}} \in \Sigma(\hat{E})$ mit $\hat{E}=\left(\left|\mu^{+}\right|+\left|\mu^{-}\right|\right) E$; außerdem erhält man $\left\|\tau_{\mu^{+} \mu^{-}}\right\| \leq$const. $\left(\mu^{+}, \mu^{-}\right)$für große $E$ und kleine $E r$, wenn man die Abschätzung der Normen der $g_{j}^{ \pm}$aus (7.3.50) berücksichtigt.

Zur Konstruktion der Weyloperatoren setzen wir für Multiindizes $\nu^{+}, \nu^{-}$:

$$
f_{\nu^{+} \nu^{-}}^{*}:=\sum_{j} \nu_{j}^{+} f_{j}^{+}+i \sum_{k} \nu_{k}^{-} f_{k}^{-} .
$$

Das liegt stets in $\mathcal{L}(r)$. Analog zu (7.3.6) betrachten wir Summen

$$
A=\sum_{\left(\nu^{+}, \nu^{-}\right) \in M} a_{\nu^{+} \nu^{-}} W\left(f_{\nu^{+} \nu^{-}}^{*}\right) \in \mathfrak{A}(r) .
$$


Dabei ist $M \subset \mathfrak{M}^{\infty} \times \mathfrak{M}^{\infty}$ eine endliche Teilmenge, die wir erst später spezifizieren werden. Die Auswertung des Funktionals $\tau_{\mu^{+} \mu^{-}}$auf $A$ ergibt wegen der Orthogonalitätsrelation (7.3.51):

$$
\begin{array}{r}
\tau_{\mu^{+} \mu^{-}}(A)=\sum_{\nu^{+}, \nu^{-}} a_{\nu^{+} \nu^{-}} e^{-\frac{1}{2}\left\|f_{\nu^{+} \nu^{-}}^{*}\right\|^{2}} \prod_{j}\left\langle\nu_{j}^{+} f_{j}^{+} \mid g_{j}^{+}\right\rangle^{\mu_{j}^{+}} \prod_{k}\left\langle\nu_{k}^{-} f_{k}^{-} \mid g_{k}^{-}\right\rangle^{\mu_{k}^{-}} \\
=: \sum_{\nu^{+}, \nu^{-}} a_{\nu^{+} \nu^{-}} C_{\mu^{+} \mu^{-} \mid \nu^{+} \nu^{-}}(E, r) .
\end{array}
$$

Setzen wir nun

$$
\Theta\left(\mu^{+}, \mu^{-}\right):=\sum_{j}\left(\mu_{j}^{+} \vartheta^{+}(j)+\mu_{j}^{-} \vartheta^{-}(j)\right)
$$

dann erhalten wir aufgrund des in (7.3.52) beschriebenen Grenzwertverhaltens:

$$
\begin{aligned}
C_{\mu^{+} \mu^{-} \mid \nu^{+} \nu^{-}} & (E r)^{-\Theta\left(\mu^{+}, \mu^{-}\right)} \\
& \underset{E \rightarrow \infty}{E r \rightarrow 0} \\
\underset{1}{\longrightarrow} & c_{1}\left(\nu^{+}, \nu^{-}\right) \cdot \prod_{j}\left(\nu_{j}^{+}\right)^{\mu_{j}^{+}} \prod_{k}\left(\nu_{j}^{-}\right)^{\mu_{j}^{-}} \cdot c_{2}\left(\mu^{+}, \mu^{-}\right)=: C_{\mu^{+} \mu^{-} \mid \nu^{+} \nu^{-}}^{0} .
\end{aligned}
$$

$c_{1}\left(\nu^{+}, \nu^{-}\right)$und $c_{2}\left(\mu^{+}, \mu^{-}\right)$sind nichtverschwindende Konstanten.

Wir fassen die Multiindizes $\mu^{+}, \mu^{-}$nun wie in Abschnitt 7.2 zu nur einem Multiindex $\mu$ zusammen (entsprechend auch $\nu^{ \pm}$) und bilden aus den $\vartheta^{ \pm}(j)$ ein entsprechendes $\vartheta(j)$, das monoton wachsen soll. $\Theta(\mu)$ wird analog definiert. Mit dieser Umbenennung lautet die Relation (7.3.58) jetzt

$$
C_{\mu \nu}(E r)^{-\Theta(\mu)} \underset{E \rightarrow \infty}{\stackrel{E r \rightarrow 0}{\longrightarrow}} c_{1}(\nu) \cdot \prod_{j} \nu_{j}^{\mu_{j}} \cdot c_{2}(\mu)=C_{\mu \nu}^{0} .
$$

Die Multiplikation mit $c_{1}(\nu)$ und $c_{2}(\mu)$ entspricht der Multiplikation des mittleren Terms mit je einer invertierbaren Diagonalmatrix. Zu betrachten bleibt die Matrix $C_{\mu \nu}^{P}:=\prod_{j} \nu_{j}^{\mu_{j}}$. Wir können diese Matrix ohne Einschränkung als invertierbar annehmen, und zwar in folgendem Sinne:

Falls $C_{\mu \nu}^{P}$ nicht invertierbar ist, dann ersetze man alle nichtverschwindenden Einträge der Multiindizes $\nu$ durch Variablen $x_{j}^{(\nu)}$ und betrachte das entsprechend gebildete $C_{\mu \nu}^{P}\left(x_{j}^{(\nu)}\right)$. Die Determinante dieser Matrix ist ein Polynom in den Variablen $x_{j}^{(\nu)}$, und zwar nicht das Nullpolynom, denn beispielsweise tritt das Monom

$$
\prod_{\nu \in M ; j}\left(x_{j}^{(\nu)}\right)^{\nu_{j}}
$$

nur in dem Term auf, der von der Diagonalen der Matrix herrührt. Mithin kann man für $x_{j}^{(\nu)}$ Werte finden (sogar in beliebiger Nähe der ursprünglichen), so daß die Determinante nicht verschwindet und damit $C_{\mu \nu}^{P}$ invertierbar wird. Startet man aber in (7.3.54) mit den geänderten Werten statt der ganzzahligen $\nu_{j}^{ \pm}$, dann verläuft die Konstruktion wie zuvor.

Insgesamt ist die Matrix $C_{\mu \nu}^{0}$ also (ohne Einschränkung) invertierbar. Da sowohl die Determinante als auch die Komponenten der inversen Matrix stetig in den Einträgen von 
$C$ sind, ist für genügend kleine $E r$ und große $E$ auch $C_{\mu \nu}(E, r)$ invertierbar, und die in Lemma 7.16 erwähnte Konstante $d$ kann gleichmäßig gewählt werden. Man kann den in Abschnitt 7.3.1 skizzierten Gedankengang also anwenden:[5 dabei wählt man den Exponenten $\bar{\gamma}$ so, daß

$$
\bar{\gamma} \geq \Theta(\mu) \quad \forall \mu \in M .
$$

Die Menge $M$ kann noch geeignet gewählt werden, um $\bar{\gamma}$ möglichst klein werden zu lassen (sie muß mindestens $N+1$ Multiindizes enthalten). Um das Ergebnis einfacher formulieren zu können, numerieren wir nun auch die $\tau_{\mu}$ und $f_{\mu}^{*}$ mit natürlichen Zahlen $j$ statt Multiindizes, wobei $\Theta(j)$ wieder monoton wachsen soll - das ist wegen $\vartheta^{ \pm}(j) \rightarrow \infty(j \rightarrow \infty)$ stets möglich. Unter Präzisierung der bisherigen Formulierung „für große $E$ und kleine $E r^{\text {“ }}$ erhalten wir dann folgende Aussage:

Satz 7.19. Wir betrachten ein Modell der freien Feldtheorie, das die Eigenschaft 7.17 besitzt, etwa die Theorie eines reellen skalaren Feldes in $s \geq 2$ Raumdimensionen. Zu $N \in \mathbb{N}$ gibt es dann Konstanten $E_{0}, w_{0}$ und $c$, so daß für $E \geq E_{0}, E r \leq w_{0}$ und beliebige Rang-N-Abbildungen $\psi_{N} \in \bar{\Psi}_{0}$ gilt:

$$
\left\|\Xi-\psi_{N}\right\|_{E, r} \geq c \cdot(E r)^{\Theta(N+1)} .
$$

Es sei bemerkt, daß die oben definierte Funktion $\Theta(j)$ mit derjenigen aus Abschnitt 7.2 übereinstimmt, vorausgesetzt, daß die $\vartheta^{ \pm}$in Eigenschaft 7.1 und Eigenschaft 7.17 ebenfalls gleich gewählt werden können (wie im Beispiel des reellen skalaren Feldes).

Wir können nun die „oberen Abschätzungen“ aus Abschnitt 7.2 und die „unteren Abschätzungen" aus diesem Abschnitt vergleichen und damit den Feldinhalt der Theorie vollständig bestimmen:

Erfüllt die betrachtete Theorie die vorausgesetzten Eigenschaften im Einteilchenraum, dann folgt zunächst aus Abschnitt 7.2, daß

$$
\operatorname{dim} \Phi_{\bar{\gamma}}<N, \quad \text { falls } \Theta(N) \geq \bar{\gamma} ;
$$

denn dann können wir nach Satz 7.5 eine Rang- $(N-1)$-Abbildung $\Psi_{N-1}$ finden, so daß $\gamma\left(\Xi-\psi_{N-1}\right) \geq \bar{\gamma}$.

Die Abschätzung in die andere Richtung folgt so: Nach Satz 4.2 wissen wir, daß

$$
\gamma\left(\left\|\Xi-p_{\bar{\gamma}} \Xi\right\|_{E, r}\right) \geq \bar{\gamma}
$$

bei festgehaltenem $E$. Dabei ist $p_{\bar{\gamma}}$ eine reguläre Projektion auf $\Phi_{\bar{\gamma}}$, weshalb $p_{\bar{\gamma}} \Xi \in \bar{\Psi}_{0}$ eine Abbildung vom Rang $\operatorname{dim} \Phi_{\bar{\gamma}}$ wird. Aus Satz 7.19 wissen wir aber, daß eine solche Approximation durch Rang- $(N-1)$-Operatoren zumindest für große $E$ nicht möglich ist, falls $\Theta(N)<\bar{\gamma}$; deshalb gilt

$$
\operatorname{dim} \Phi_{\bar{\gamma}} \geq N, \quad \text { falls } \Theta(N)<\bar{\gamma} .
$$

Aus (7.3.61) und (7.3.63) erhält man unmittelbar, daß

$$
d(\bar{\gamma}) \equiv \operatorname{dim} \Phi_{\bar{\gamma}}=\max \{N \mid \Theta(N)<\bar{\gamma}\} .
$$

15 Dazu müssen die Funktionale $\tau_{\mu}$ noch normiert und die Energieskala um einen konstanten Faktor geändert werden. Dies läßt sich aber in den im Endergebnis erwähnten Konstanten auffangen. 
Man sieht hieran noch einmal explizit die linksseitige Stetigkeit von $d(\bar{\gamma})$.

Damit haben wir nicht nur $\operatorname{dim} \Phi_{\bar{\gamma}}$ bestimmt, sondern auch den Raum $\Phi_{\bar{\gamma}}$ selbst: Da per Definition

$$
\Theta(d(\bar{\gamma})+1) \geq \bar{\gamma}
$$

liefert uns Satz 7.5 eine Abbildung $\psi$ vom Rang $\operatorname{dim} \Phi_{\bar{\gamma}}$, die $\gamma(\Xi-\psi) \geq \bar{\gamma}$ erfüllt und deren Rechts-Bild daher mit $\Phi_{\bar{\gamma}}$ übereinstimmt. Explizit hat man

$$
\Phi_{\bar{\gamma}}=\operatorname{Span}\left\{\phi_{j}\right\}_{j=1}^{d(\bar{\gamma})}
$$

die in Abschnitt 7.2 berechneten $\phi_{j}$ bilden also gerade den Feldinhalt der Theorie.

Ein explizites Beispiel für die ersten Terme der Approximationsreihe und für die zugehörigen $\Theta(N)$ und $d(\bar{\gamma})$ ist in Abschnitt 7.4 .2 angegeben.

\subsection{A Ein Hilfssatz im Einteilchenraum}

Im Zusammenhang mit unserer Analyse im Einteilchenraum der Theorie betrachten wir folgenden Ausdruck: Sei $\kappa$ ein $s$-stelliger Multiindex, dann ist

$$
\Delta(\kappa):=\int_{|\vec{x}|=1} d \Omega(x) x^{\kappa}
$$

das Integral läuft über die Einheitssphäre im $\mathbb{R}^{s}$. Wir bemerken folgende Eigenschaften:

$$
\begin{gathered}
\Delta(\kappa) \geq 0 \\
\Delta(\kappa)=0 \Leftrightarrow \kappa \text { besitzt einen ungeraden Eintrag, } \\
\text { insbesondere: } \Delta(2 \kappa)>0 .
\end{gathered}
$$

Das folgende Lemma stellt sich für unsere Anwendungen als wichtig heraus.

Lemma 7.20. Sei $M \subset \mathfrak{M}^{s}$ eine endliche Teilmenge; dann ist die Matrix

$$
B_{\kappa \lambda}=\frac{\Delta(\kappa+\lambda)}{s+|\kappa|+|\lambda|}, \quad \kappa, \lambda \in M
$$

positiv definit, insbesondere ist sie invertierbar.

Beweis. Man betrachte die Funktionen

$$
f_{\kappa}(\vec{x})=x^{\kappa} \chi_{[0,1]}(|\vec{x}|)=\left\{\begin{array}{ll}
x^{\kappa} & \text { für }|\vec{x}| \leq 1, \\
0 & \text { sonst; }
\end{array} \quad \kappa \in M .\right.
$$

Sie sind offenbar linear unabhängig. Bezeichnet $\langle\cdot \mid \cdot\rangle$ das gewöhnliche $L^{2}\left(\mathbb{R}^{s}\right)$-Skalarprodukt, dann gilt

$$
\left\langle f_{\kappa} \mid f_{\lambda}\right\rangle=B_{\kappa \lambda}
$$

wie man leicht berechnet. $B_{\kappa \lambda}$ ist also die Matrixdarstellung des Skalarprodukts auf dem Teilraum $\operatorname{Span}\left\{f_{\kappa}\right\}$ und deshalb positiv definit. 


\subsection{Freie Wightman-Felder}

Wir haben bisher das betrachtete Modell der freien Feldtheorie vollständig im algebraischen Rahmen formuliert und das Verhalten der Theorie „am Punkt" analysiert, wodurch wir dann in Kapitel 3 lokale Punktfelder am Koordinatenursprung erhalten haben. Historisch wurde die freie Feldtheorie aber zuerst durch Punktfelder (bzw. durch "ausgeschmierte“ operatorwertige Distributionen) definiert und später auf die algebraische Theorie übertragen.

Wir geben in diesem Abschnitt zunächst einen Überblick über die Formulierung der freien Feldtheorie im Wightman'schen Rahmen und zeigen, wie die algebraische Sichtweise daraus hervorgeht. Dabei beschränken wir uns auf das reelle skalare Feld; nur dort hatten wir den Einteilchenraum und die „lokalen“ Unterräume $\mathcal{L}^{ \pm}(r)$ konkret definiert. Anschließend geben wir die ersten Terme der in Abschnitt 7.2 .6 hergeleiteten Reihenentwicklung explizit an; wir deuten die darin vorkommenden quadratischen Formen als Punktfelder und zeigen, daß man nach Ausintegration tatsächlich das Feld $\phi(f)$ zurückerhält, mit dessen Hilfe die Theorie definiert wurde.

\subsubsection{Das reelle skalare Feld im Wightman-Rahmen}

Ein reelles skalares Feld ist in der Wightman'schen Quantenfeldtheorie eine hermitesche operatorwertige Distribution

$$
\phi(f)^{*} \supset \phi(\bar{f}), \quad f \in \mathcal{S}(\mathcal{M})
$$

Das Feld $\phi$ soll (im Sinne von Distributionen) die Klein-Gordon-Gleichung erfüllen:

$$
\left(\square+m^{2}\right) \phi=0 .
$$

Genügt ein solches Feld allen in Abschnitt 1.3.2 aufgeführten Wightman-Axiomen, einschließlich der dort genannten Vollständigkeitsbedingung, dann erfüllt es notwendigerweise [Rob62] die Vertauschungsrelationen

$$
[\phi(f), \phi(g)]=\left(\langle f \mid g\rangle_{+}-\langle g \mid f\rangle_{+}\right) \cdot \mathbf{1}
$$

mit

$$
\langle f \mid g\rangle_{+}=\int d^{s+1} x \int d^{s+1} y f(x) g(y) \Delta_{+}(x-y)
$$

hierbei ist $\Delta_{+}$die Distribution

$$
\Delta_{+}(z)=(2 \pi)^{-s} \int d^{s} p \delta\left(p^{2}-m^{2}\right) \theta\left(p_{0}\right) e^{-i p z} .
$$

Dadurch ist das Feld $\phi(f)$ bis auf Unitäräquivalenz eindeutig festgelegt. Es kann in der üblichen Fockraumdarstellung konstruiert werden [\$0s65]; dort läßt es sich in die bekannten Erzeugungs- und Vernichtungsoperatoren $a^{*}$ und a zerlegen:

$$
\phi(f)=a^{*}(f)+a(f), \quad f \in \mathcal{S}_{\mathbb{R}}(\mathcal{M}) .
$$


Um die lokalen Algebren $\mathfrak{A}(\mathcal{O})$ zu erhalten, muß man beschränkte Funktionen der $\phi(f)$ betrachten. Da die $\phi(f)$ für reellwertiges $f$ sogar (wesentlich) selbstadjungiert sind, bieten sich dazu die unitären Weyl-Operatoren

$$
W(f)=e^{i \phi(f)}, \quad f \in \mathcal{S}_{\mathbb{R}}(\mathcal{M})
$$

an..$^{[6]}$ Sie erfüllen wegen (7.4.3) die Relation

$$
W(f) W(g)=e^{-i \operatorname{Im}\langle f \mid g\rangle_{+}} W(f+g) .
$$

Die in $\mathcal{O}$ lokalisierten Observablen erhält man nun, indem man die im Ortsraum im Gebiet $\mathcal{O}$ lokalisierten reellwertigen Testfunktionen $f$ und Funktionen der zugehörigen Felder $\phi(f)$ betrachtet, also

$$
\mathfrak{A}(\mathcal{O})=\left\{W(f) \mid f \in \mathcal{D}_{\mathbb{R}}(\mathcal{O})\right\}^{\prime \prime} .
$$

Es ist dabei aber nicht notwendig, $f$ tatsächlich durch ganz $\mathcal{D}_{\mathbb{R}}(\mathcal{O})$ laufen zu lassen. Man bemerkt nämlich

$$
\phi\left(\left(\square+m^{2}\right) f\right)=\left(\left(\square+m^{2}\right) \phi\right)(f) \stackrel{([\overrightarrow{r .4 .2})}{=} 0 \quad \forall f \in \mathcal{D}_{\mathbb{R}}(\mathcal{M}) ;
$$

folglich reicht es aus, Restklassen $[f]$ aus dem Quotientenraum $\mathcal{D}_{\mathbb{R}}(\mathcal{O}) /\left(\square+m^{2}\right) \mathcal{D}_{\mathbb{R}}(\mathcal{M})$ zu betrachten. Einem solchen $[f]$ können wir seine $s$-dimensionale Fouriertransformierte zuordnen:

$$
\tilde{f}(\vec{p}):=(2 \pi)^{-s / 2} \int d^{s+1} x f(x) e^{i\left(\omega(\vec{p}) x^{0}-\vec{p} \vec{x}\right)} \quad\left(\omega(\vec{p})=\sqrt{\vec{p}^{2}+m^{2}}\right) .
$$

Diese Zuordnung $[f] \mapsto \tilde{f}$ ist wohldefiniert und injektiv, denn für $f \in \mathcal{D}_{\mathbb{R}}(\mathcal{M})$ gilt

$$
\int d^{s+1} x f(x) e^{i\left(\omega x^{0}-\vec{p} \vec{x}\right)}=0 \forall \vec{p} \quad \Leftrightarrow \quad f \in\left(\square+m^{2}\right) \mathcal{D}_{\mathbb{R}}(\mathcal{M}) .
$$

(Die Richtung „ $\Rightarrow$ “ folgt dabei mit Hilfe funktionentheoretischer Argumente - man beachte, daß wegen der Trägereigenschaften von $f$ seine $(s+1)$-dimensionale Fouriertransformierte ganz analytisch ist.)

Wir zerlegen $\tilde{f}$ dann in $J$-invariante Funktionen ( $J$ wird wie in (7.1.5) definiert):

$$
\tilde{f}=\tilde{f}^{+}+i \omega \tilde{f}^{-} \quad \text { mit } \quad \tilde{f}^{+}=\frac{1}{2}(\tilde{f}+J \tilde{f}), \tilde{f}^{-}=\frac{1}{2 i \omega}(\tilde{f}-J \tilde{f}) ;
$$

diese Zerlegung ist eindeutig. Wichtig ist nun, daß sich die Lokalisierung von $f$ an $\operatorname{den} \tilde{f}^{ \pm}$ ablesen läßt: Es sei speziell $\mathcal{O}=\mathcal{O}_{r}$ ein Standard-Doppelkegel; dann gehören vorgegebene $J$-invariante Testfunktionen $\tilde{f}^{ \pm}$genau dann zu einem $[f] \in \mathcal{D}_{\mathbb{R}}\left(\mathcal{O}_{r}\right) /\left(\square+m^{2}\right) \mathcal{D}_{\mathbb{R}}(\mathcal{M})$, wenn die Träger der Fourierrücktransformierten

$$
f^{ \pm}(\vec{x})=(2 \pi)^{-\frac{s}{2}} \int d^{s} p e^{i \vec{p} \vec{x}} \tilde{f}^{ \pm}(\vec{p})
$$

\footnotetext{
${ }^{16}$ Die Einschränkung auf reellwertige Testfunktionen bedeutet keinen „Verlust an Information“, da sich jede komplexwertige Funktion als Linearkombination zweier reellwertiger schreiben läßt.
} 
innerhalb der Kugel $|\vec{x}|<r$ liegen. Wir erhalten also eine eineindeutige Zuordnung $[f] \mapsto$ $\tilde{f}=\tilde{f}^{+}+i \omega \tilde{f}^{-}$, wobei die $\tilde{f}^{ \pm}$die genannten Eigenschaften haben. Betrachtet man das Feld nun als Funktion von $\tilde{f}$ statt $[f]$, dann lautet die Weylrelation (7.4.8)

$$
W(\tilde{f}) W(\tilde{g})=e^{-i \operatorname{Im}\langle\tilde{f} \mid \tilde{g}\rangle} W(\tilde{f}+\tilde{g})
$$

mit

$$
\langle\tilde{f} \mid \tilde{g}\rangle=\int \frac{d^{s} p}{2 \omega(\vec{p})} \bar{f}(\vec{p}) \tilde{g}(\vec{p})=\langle f \mid g\rangle_{+} .
$$

Zur Vereinfachung der Rechnungen ziehen wir den Faktor $(2 \omega)^{-1}$ im Integrationsmaß noch zu den Funktionen, d.h. wir setzen

$$
\hat{f}(\vec{p}):=(2 \omega)^{-\frac{1}{2}} \tilde{f}(\vec{p})
$$

und erhalten

$$
W(\hat{f}) W(\hat{g})=e^{-i \operatorname{Im}\langle\hat{f} \mid \hat{g}\rangle} W(\hat{f}+\hat{g}) \quad \operatorname{mit} \quad\langle\hat{f} \mid \hat{g}\rangle=\int d^{s} p \overline{\hat{f}(\vec{p})} \hat{g}(\vec{p})
$$

auf eine Neubezeichnung des Skalarprodukts verzichten wir dabei. Die lokale Algebra $\mathfrak{A}(r)$ wird nun erzeugt von den Weyl-Operatoren

$$
W(\hat{f}), \quad \hat{f}=\omega^{-\frac{1}{2}} \hat{f}^{+}+i \omega^{\frac{1}{2}} \hat{f}^{-},
$$

wobei $\hat{f}^{ \pm}$im Ortsraum reell und in $|\vec{x}|<r$ lokalisiert sind. Das stimmt bis auf Bildung topologischer Abschlüsse mit unserer bisherigen Definition in Abschnitt 7.1 überein.

\subsubsection{Rekonstruktion des Feldes}

Wir hatten unter gewissen Voraussetzungen im Einteilchenraum in Abschnitt 7.2 eine Reihenentwicklung der Abbildung $\Xi$ hergeleitet,

$$
\Xi=\sum_{j} \phi_{j} \sigma_{j}
$$

und in Abschnitt 7.3 gezeigt, daß die $\phi_{j}$ den Feldinhalt der Theorie bilden. Für das reelle skalare Feld hatten wir die Einteilchenraum-Eigenschaften explizit nachgeprüft. Es ist instruktiv, die ersten Terme dieser Reihe explizit auszurechnen. Um die Notation aus Abschnitt 7.2 etwas zu vereinfachen, setzen wir $h=h_{\kappa=0}^{+}$und $g=g_{\kappa=0}^{+}$. Man erhält dann im 
Fall physikalischer Raumzeit:ㅍ

$$
\begin{array}{rlr}
\Xi_{E, r}= & (\Omega|\cdot| \Omega) \\
& +\frac{1}{2}((h|\cdot| \Omega)+(\Omega|\cdot| h)) & \cdot P(E)\left(a(g)+a^{*}(g)\right) P(E) \\
& +\frac{1}{2} \sum_{j=1}^{3}\left(\left(x_{j} h|\cdot| \Omega\right)+\left(\Omega|\cdot| x_{j} h\right)\right) \cdot P(E)\left(a\left(-i p_{j} g\right)+a^{*}\left(-i p_{j} g\right)\right) P(E) \\
& +\frac{1}{2 i}\left(\left(\omega^{-1} h|\cdot| \Omega\right)-\left(\Omega|\cdot| \omega^{-1} h\right)\right) \cdot P(E)\left(a(i \omega g)+a^{*}(i \omega g)\right) P(E) \\
& +\sigma_{Q}(\cdot) & \cdot P(E)\left(a(g)^{2}+a^{*}(g)^{2}+2 a^{*}(g) a(g)\right) P(E) \\
& +\ldots
\end{array}
$$

Die Schreibweise $x_{j} h$ ist dabei etwas symbolisch und müßte genauer $\omega^{-\frac{1}{2}} x_{j} \omega^{+\frac{1}{2}} h$ heißen. Das mit $\sigma_{Q}$ abgekürzte lineare Funktional lautet explizit

$$
\sigma_{Q}(\cdot)=\frac{1}{4 \sqrt{2}}((h \otimes h|\cdot| \Omega)+(\Omega|\cdot| h \otimes h))+\frac{1}{4}(h|\cdot| h)-\frac{\|h\|^{2}}{4}(\Omega|\cdot| \Omega) .
$$

Intuitiv erkennt man in den Approximationstermen bereits das Punktfeld $\phi(0)$ wieder; in etwas suggestiver Schreibweise lautet die obige Gleichung

$$
\begin{aligned}
& \Xi=(\Omega|\cdot| \Omega) \quad \cdot \mathbf{1} \quad(\gamma=0) \\
& +\frac{1}{2}((h|\cdot| \Omega)+(\Omega|\cdot| h)) \quad \cdot \phi(0) \quad(\gamma=1) \\
& +\frac{1}{2} \sum_{j=1}^{3}\left(\left(x_{j} h|\cdot| \Omega\right)+\left(\Omega|\cdot| x_{j} h\right)\right) \cdot \partial_{j} \phi(0) \quad(\gamma=2) \\
& +\frac{1}{2 i}\left(\left(\omega^{-1} h|\cdot| \Omega\right)-\left(\Omega|\cdot| \omega^{-1} h\right)\right) \cdot \partial_{t} \phi(0) \quad(\gamma=2) \\
& +\sigma_{Q}(\cdot) \quad \cdot: \phi^{2}:(0) \quad(\gamma=2) \\
& +\ldots
\end{aligned}
$$

Wir haben hier explizit $\operatorname{dim} \Phi_{1}=1, \operatorname{dim} \Phi_{2}=2, \operatorname{dim} \Phi_{3}=7, \Theta\left(N_{j}+1\right)=j$. Die Terme zu $\gamma \geq 3$ sind nicht aufgeführt. In der Reihenentwicklung treten neben dem Feld $\phi$ auch seine räumlichen und zeitlichen Ableitungen sowie das Wickprodukt : $\phi^{2}$ : auf. Bei den höheren (nicht gezeigten) Termen handelt es sich entsprechend um höhere Ableitungen des Feldes (auch gemischte räumliche und zeitliche) sowie deren Wickprodukte. Zweite oder höhere Zeitableitungen treten allerdings nicht auf - das ist Ausdruck der Feldgleichung (7.4.2).

Man beachte, daß die hier aufgeschriebenen ersten Terme der Reihenentwicklung mit dem Ergebnis der heuristischen Rechnung in [H()96] im wesentlichen übereinstimmen; die Form der Funktionale $\sigma_{j}$ ist jedoch aufwendiger als dort vermutet.

Die Schreibweise $a^{*}(g)+a(g)=\phi(0)$ usw., zu verstehen im Sinne von Linearformen auf $\bar{\Sigma}$, ist zwar heuristisch unmittelbar einleuchtend; streng genommen bleibt aber noch zu

\footnotetext{
17 Für $s>3$ ändert sich die Reihenfolge der Terme; der Term mit dem Wick-Quadrat des Feldes gehört zu $\gamma=s-1$, während die Terme mit den ersten Ableitungen $\gamma=1+\frac{s-1}{2}$ aufweisen.
} 
zeigen, daß die aus den $\phi_{j}$ nach Integration (Abschnitt 3.4) erhaltenen Wightman-Felder tatsächlich mit dem definierenden Feld $\phi(f)$ und seinen Ableitungen übereinstimmen. Wir führen den Beweis hier für das Feld selbst (2. Term der Entwicklung (7.4.23) ); für die Ableitungen und Wickprodukte läßt sich eine analoge Argumentation durchführen. Im folgenden sei wieder $s \geq 3$ beliebig.

$\mathrm{Zu}$ zeigen ist die Gleichheit der quadratischen Form $a^{*}(g)+a(g)$ mit dem Feld $\phi(f)$ nach Ausintegration mit einer reellwertigen Testfunktion $f$, und zwar im Sinne von Operatoren auf $\mathcal{C}^{\infty}(\mathcal{H})$. Es reicht jedoch aus, die Identität auf einer dichten Menge im Sinne von quadratischen Formen nachzuprüfen. Seien also

$$
\xi=b_{1} \otimes \cdots \otimes b_{m} \in P(E) \mathcal{H}, \quad \xi^{\prime}=c_{1} \otimes \cdots \otimes c_{n} \in P(E) \mathcal{H}
$$

dabei sind $b_{j}, c_{k} \in Q(E) \mathcal{K}$. Wir haben zu zeigen, daß

$$
\int\left(\xi \mid U(x)\left(a^{*}(g)+a(g)\right) U(x)^{*} \xi^{\prime}\right) f(x) d^{s+1} x=\left(\xi \mid \phi(f) \xi^{\prime}\right) .
$$

Schreibt man die rechte Seite nach (7.4.6) als Erzeuger und Vernichter aus, dann reicht es, folgendes nachzuweisen:

$$
\int d^{s+1} x f(x)\left(U(x)^{*} \xi \mid a^{\sharp}(g) U(x)^{*} \xi^{\prime}\right)=\left(\xi \mid a^{\sharp}(\hat{f}) \xi^{\prime}\right) .
$$

Dabei ist $a^{\sharp}$ entweder $a$ oder $a^{*}$; wir behandeln den Fall $a^{\sharp}=a^{*}$, der andere ergibt sich entsprechend. Interessant ist dann nur $m=n+1$, andernfalls verschwinden beide Seiten von (7.4.26). Man hat nun

$$
\begin{gathered}
\int d^{s+1} x f(x)\left(U(x)^{*} \xi \mid a^{*}(g) U(x)^{*} \xi^{\prime}\right) \\
=\int d^{s+1} x f(x)\left(\operatorname{Symm} \otimes_{j} U_{\mathcal{K}}(-x) b_{j} \mid a^{*}(g) \operatorname{Symm} \otimes_{k} U_{\mathcal{K}}(-x) c_{k}\right) \\
\stackrel{\sqrt{7.1 .10}}{=} \sqrt{n+1} \int d^{s+1} x f(x)\left(\operatorname{Symm} \otimes_{j} U_{\mathcal{K}}(-x) b_{j} \mid \operatorname{Symm}\left(g \otimes \otimes_{k} U_{\mathcal{K}}(-x) c_{k}\right)\right) \\
=\sqrt{n+1} \operatorname{Symm}\left(\prod_{j=1}^{n}\left\langle b_{j} \mid c_{j}\right\rangle \cdot \int d^{s+1} x f(x) \int d^{s} p e^{i\left(\omega x^{0}-\vec{p} \vec{x}\right)} \overline{b_{n+1}(\vec{p})}(2 \pi)^{-\frac{s}{2}}(2 \omega)^{-\frac{1}{2}} \chi_{E}(\vec{p})\right) .
\end{gathered}
$$

Die Symmetrisierung läuft dabei über die Indizes der $b_{j}$. Da das Integral über $d^{s} p$ nur über ein endliches Gebiet läuft und der Integrand auch bezüglich $x$ genügend schnell abfällt $(f$ ist Testfunktion), kann die Integrationsreihenfolge vertauscht werden. Außerdem kann die Multiplikation mit $\chi_{E}(\vec{p})$ entfallen, da bereits $b_{n+1} \in Q(E) \mathcal{K}$. Man erhält so

$$
\begin{aligned}
& \int d^{s+1} x f(x)\left(U(x)^{*} \xi \mid a^{*}(g) U(x)^{*} \xi^{\prime}\right) \\
& =\sqrt{n+1} \operatorname{Symm}\left(\prod_{j=1}^{n}\left\langle b_{j} \mid c_{j}\right\rangle \cdot \int d^{s} p \overline{b_{n+1}(\vec{p})} \hat{f}(\vec{p})\right) \\
& =\sqrt{n+1} \operatorname{Symm}\left(\prod_{j=1}^{n}\left\langle b_{j} \mid c_{j}\right\rangle \cdot\left\langle b_{n+1} \mid \hat{f}\right\rangle\right)=\left(\xi \mid a^{*}(\hat{f}) \xi^{\prime}\right),
\end{aligned}
$$


wobei

$$
\hat{f}(\vec{p})=(2 \omega)^{-\frac{1}{2}}(2 \pi)^{-\frac{s}{2}} \int d^{s+1} x f(x) e^{i\left(\omega x^{0}-\vec{p} \vec{x}\right)} .
$$

Damit ist schließlich gezeigt, daß

$$
\int d^{s+1} x f(x) U(x)\left(a^{*}(g)+a(g)\right) U(x)^{*}=\phi(f)
$$

im bereits diskutierten Sinne.

\subsection{Produktentwicklung und Normalproduktraum}

Um die Berechnung der Operatorproduktentwicklung zu illustrieren, betrachten wir wiederum den Fall eines reellen skalaren freien Feldes in $s=3$ räumlichen Dimensionen. Wir untersuchen dort das "Quadrat" des Feldes, also das Produkt $\Pi=\phi \otimes \phi$.

Dazu benötigen wir zunächst eine reguläre Projektion $p_{\bar{\gamma}}$ auf $\Phi_{\bar{\gamma}}$, wobei $\bar{\gamma}$ geeignet groß gewählt werden muß. Wir werden unten explizit sehen, daß $\bar{\gamma}=3$ für die Operatorproduktentwicklung zur Genauigkeit $\beta=0$ ausreichend ist. Im Raum $\Phi_{3}$ wählen wir die Basis

$$
\left\{\phi_{j}\right\}_{j=1}^{7}=\left\{\mathbf{1}, \phi, \partial_{1} \phi, \partial_{2} \phi, \partial_{3} \phi, \partial_{t} \phi,: \phi^{2}:\right\}
$$

Um die gewünschte Projektion zu konstruieren, müssen wir energiebeschränkte Funktionale $\sigma_{j}$ finden, so daß

$$
\sigma_{j}\left(\phi_{k}\right)=\delta_{j k}
$$

Heuristisch liegt es nahe, hierzu die in (7.4.23) aufgeführten linearen Funktionale zu verwenden. Tatsächlich stellt man fest, daß sie $\sigma_{j}\left(\phi_{k}\right)=\delta_{j k}$ erfüllen. Sie sind jedoch nicht energiebeschränkt, da die Funktion $\omega^{-\frac{1}{2}} h$ im Ortsraum kompakten Träger besitzt.18 Wir werden deshalb die Funktion $h$ modifizieren und wählen dazu eine Testfunktion $f \in \mathcal{S}\left(\mathbb{R}^{s}\right)$ mit kompaktem Träger, so daß

$$
\int f(\vec{p}) d^{s} p=(2 \pi)^{\frac{s}{2}}, \quad \int p_{j} f(\vec{p}) d^{s} p=0 \quad \forall j \in\{1, \ldots, s\} .
$$

(Das kann man z.B. mit einer geeigneten rotationssymmetrischen Funktion $f$ sicher erreichen.) Wir setzen dann

$$
\check{h}(\vec{p})=(2 \omega)^{\frac{1}{2}} f(\vec{p})
$$

und bezeichnen mit $\check{\sigma}_{j}$ die Funktionale, die man aus $\sigma_{j}$ erhält, indem man $h$ durch $\check{h}$ ersetzt, also

$$
\check{\sigma}_{1}=(\Omega|\cdot| \Omega), \quad \check{\sigma}_{2}=\frac{1}{2}((\check{h}|\cdot| \Omega)+(\Omega|\cdot| \check{h})), \quad \ldots, \quad \check{\sigma}_{7}=\check{\sigma}_{Q} .
$$

\footnotetext{
18 Zwar konvergiert $h$ im Limes eines großen „Abschneideradius“ gegen die Delta-Funktion im Impulsraum, aber in diesem Limes bleibt die Norm von $h$ nicht beschränkt. Rechnet man trotzdem formal mit dem „nicht regulären“ Projektor $\sum_{j} \sigma_{j}(\cdot) \phi_{j}$, dann erhält man zwar endliche Ergebnisse, in höheren Ordnungen der Produktentwicklung ergeben sich jedoch Widersprüche zur Lorentz-Kovarianz der Koeffizientenfunktionen.
} 
Mit Hilfe der Relationen

$$
\langle\check{h} \mid g\rangle=1, \quad\left\langle x_{j} \check{h} \mid-i p_{k} g\right\rangle=\delta_{j k}, \quad\left\langle\check{h} \mid-i p_{j} g\right\rangle=0=\left\langle x_{j} \check{h} \mid g\right\rangle
$$

rechnet man leicht nach, daß tatsächlich

$$
\check{\sigma}_{j}\left(\phi_{k}\right)=\delta_{j k}
$$

also ist $p_{\bar{\gamma}}=\sum_{j} \check{\sigma}_{j}(\cdot) \phi_{j}$ eine Projektion mit den gewünschten Eigenschaften.

Wir betrachten nun das raumartige Produkt

$$
\Pi(x, y)=\phi(x) \phi(y)=U(x) \phi U(y-x) \phi U(-y) \quad\left(x, y \in \mathcal{M},(x-y)^{2}<0\right),
$$

das man als holomorphe Fortsetzung in der Variablen $z$ des Produkts

$$
\Pi_{0}(z)=U(x) \phi U(z) \phi U(-z-x) \quad\left((\operatorname{Re} z)^{2}<0, \operatorname{Im} z \in V_{+}\right)
$$

erhält (im Sinne von Linearformen auf $\bar{\Sigma}$ ). Wir berechnen $p_{\bar{\gamma}} \Pi(x, y)$ und setzen dazu $\Pi_{0}(z)$ in die Funktionale $\check{\sigma}_{j}$ ein. Eine kurze Rechnung zeigt, daß

$$
\left(\Omega\left|\Pi_{0}(z)\right| \Omega\right)=\Delta_{+}(-z)
$$

was tatsächlich eine Fortsetzung auf reelle raumartige $z=y-x$ besitzt (siehe (7.5.17) unten). Weiter stellt man fest, daß

$$
\sigma_{j}\left(\Pi_{0}(z)\right)=0 \text { für } j \in\{2, \ldots, 6\}
$$

und daß nach Fortsetzung gilt

$$
\begin{aligned}
\check{\sigma}_{Q}(\Pi(x, y))= & \frac{1}{4}\left\langle\check{h} \mid e^{i p x} g\right\rangle\left\langle\check{h} \mid e^{i p y} g\right\rangle+\frac{1}{4}\left\langle e^{i p x} g \mid \check{h}\right\rangle\left\langle\check{h} \mid e^{i p y} g\right\rangle \\
& +\frac{1}{4}\left\langle\check{h} \mid e^{i p x} g\right\rangle\left\langle e^{i p y} g \mid \check{h}\right\rangle+\frac{1}{4}\left\langle e^{i p x} g \mid \check{h}\right\rangle\left\langle e^{i p y} g \mid \check{h}\right\rangle \\
= & \operatorname{Re}\left(\left\langle\check{h} \mid e^{i p x} g\right\rangle\right) \cdot \operatorname{Re}\left(\left\langle\check{h} \mid e^{i p y} g\right\rangle\right) .
\end{aligned}
$$

Hierbei hat man

$$
\left\langle\check{h} \mid e^{i p x} g\right\rangle=(2 \pi)^{-\frac{s}{2}} \int f(\vec{p}) e^{i p x} d^{s} p .
$$

Da dieses Integral nur über ein kompaktes Gebiet läuft, sieht man explizit, daß eine holomorphe Fortsetzung von (7.5.12) auf beliebige $x \in \mathbb{C}^{s+1}$ existiert (ebenso in $y$ ). Weiter folgt nach dem Satz von der majorisierten Konvergenz

$$
\left\langle\check{h} \mid e^{i p x} g\right\rangle \underset{x \rightarrow 0}{\longrightarrow}\langle\check{h} \mid g\rangle=1
$$

weshalb wir erhalten:

$$
\check{\sigma}_{Q}(\Pi(x, y)) \underset{x, y \rightarrow 0}{\longrightarrow} 1 .
$$


Die Produktentwicklung lautet damit insgesamt

$$
p_{\bar{\gamma}} \Pi(x, y)=\Delta_{+}(x-y) \cdot \mathbf{1}+\rho(x, y) \cdot: \phi^{2}:
$$

mit einer Funktion $\rho(x, y)$, die für $x, y \rightarrow 0$ gegen 1 konvergiert; der Faktor $\Delta_{+}(x-y)$ läßt sich auch schreiben als

$$
\Delta_{+}(x-y)=\left\{\begin{array}{ll}
\frac{m}{4 \pi^{2} \sqrt{-(x-y)^{2}}} K_{1}\left(m \sqrt{-(x-y)^{2}}\right) & (m>0), \\
-\frac{1}{4 \pi^{2}(x-y)^{2}} & (m=0)
\end{array} \text { für }(x-y)^{2}<0,\right.
$$

woraus man die Divergenz des Koeffizienten am Koordinatenursprung ablesen kann. $\left(K_{1}(z)\right.$ ist hier eine modifizierte Besselfunktion.)

Wir wollen nun explizit zeigen, daß $p_{\bar{\gamma}} \Pi(x, y)$ das Produkt $\Pi$ im raumartigen Limes approximiert. Dazu schreiben wir per Wick-Ordnung

$$
\begin{aligned}
\Pi(x, y) & =: \phi(x) \phi(y):+(\Omega|\phi(x) \phi(y)| \Omega) \cdot \mathbf{1} \\
& =: \phi(x) \phi(y):+\Delta_{+}(x-y) \cdot \mathbf{1}
\end{aligned}
$$

wobei nach dem Wick'schen Theorem gilt

$$
: \phi(x) \phi(y): \quad \underset{\mathrm{R}}{\longrightarrow}: \phi^{2}:
$$

Zusammen mit (7.5.16) folgt daraus offenbar

$$
\left\|\Pi(x, y)-p_{\bar{\gamma}} \Pi(x, y)\right\|_{E} \underset{\mathrm{R}}{\longrightarrow} 0 \quad \forall E>0,
$$

d.h. $\bar{\gamma}=3$ war genügend groß gewählt, und die Operatorproduktentwicklung ist konsistent mit der Wick-Ordnung.

Aus (7.5.16) läßt sich auch der Normalproduktraum von $\Pi=\phi \otimes \phi$ ablesen: Er liegt sicher in der linearen Hülle von 1 und : $\phi^{2}$ : ; andererseits gibt es offenbar keinen eindimensionalen Raum, der für $\phi \otimes \phi$ raumartig approximierend ist, weshalb wir

$$
\mathrm{N}[\phi \otimes \phi]=\operatorname{Span}\left\{\mathbf{1}, \phi^{2}:\right\}
$$

erhalten. 



\section{Kapitel 8}

\section{Erweiterungen und Ausblick}

Die im vorigen Kapitel analysierte freie Feldtheorie zeigt die prinzipielle Anwendbarkeit unseres Verfahrens zur Punktfeldanalyse. Wünschenswert ist aber eine Anwendung des Formalismus auf physikalisch realistischere Modelle. Wir diskutieren hier, inwieweit die in der freien Feldtheorie gefundene Situation auch auf wechselwirkende Modelle extrapoliert werden kann, und welche Aussagen sich unabhängig von konkreten Modellen - nur aufgrund der allgemeinen Eigenschaften einer relativistischen Quantentheorie und des Phasenraumkriteriums - treffen lassen.

Wir behandeln dazu zunächst Verallgemeinerungen des in Kapitel 7 entwickelten Verfahrens für das reelle skalare freie Feld. Auf diese Weise untersuchen wir unter anderem, ob der mathematische Rahmen auch Teilchen von höherem Spin, die Behandlung von Fermionen oder die Analyse von Modellen in niedrigen Raum-Zeit-Dimensionen zuläßt. Wir diskutieren kurz ein Netz von Algebren, das nicht aus Punktfeldern aufgebaut ist und trivialen Feldinhalt besitzt [L.1.97].

Weiter führen wir aus, unter welchen physikalischen Voraussetzungen das Phasenraumkriterium in Modellen gültig sein sollte, und welche Konsequenzen daraus für die Struktur der Theorie folgen. Wir skizzieren ein Kriterium, um Theorien, die von lokalen Punktfeldern erzeugt werden, von solchen mit nicht punktartig lokalisierten Observablen zu unterscheiden: Ist der Feldinhalt „ausreichend groß“, dann kann das lokale Netz vollständig aus ihm rekonstruiert werden. Dies liefert eine Charakterisierung von Eichtheorien, die sich allein auf die Betrachtung observabler Größen stützt. 


\subsection{Weitere Aspekte der freien Theorie}

Aufbauend auf der Behandlung des reellen skalaren freien Feldes in Kapitel 7 untersuchen wir die Übertragbarkeit der Analyse auf weitere freie Theorien, insbesondere solche mit anderem Einteilchenraum und in anderen Raum-Zeit-Dimensionen.

Allgemeine freie bosonische Modelle Es stellt sich zunächst die Frage, ob das Phasenraumkriterium auch in Theorien mit anderer Teilchenstruktur als der relativ simplen des reellen skalaren Feldes etabliert werden kann. Hierzu bemerkt man, daß sowohl der Nachweis des Phasenraumkriteriums wie auch die Bestimmung des Feldinhalts auf gewisse Eigenschaften im Einteilchenraum der Theorie zurückgeführt wurden; sind diese Eigenschaften gegeben, dann läuft das folgende Konstruktionsverfahren („zweite Quantisierung“") unabhängig von den Details des Modells. Man sieht leicht, daß die besagten Eigenschaften 7.1, 7.2 und 7.17 bei der Bildung direkter Summen von Einteilchenräumen erhalten bleiben; auf diese Weise läßt sich unsere Analyse also auch auf Theorien mit

- mehreren (jedoch endlich vielen) Teilchensorten,

- Bosonen von höherem Spin,

- geladenen Teilchen (komplexe Felder)

übertragen.

Allerdings ist das asymptotische Phasenraumkriterium in Theorien mit unendlich vielen Teilchensorten, unabhängig von deren Masse, nicht erfüllt. Während andere in der Literatur bekannte Phasenraumbedingungen solche Theorien zumindest teilweise noch zulassen [BP90], fallen sie aus unserem Rahmen aufgrund ihres Kurzabstandsverhaltens heraus: Mit Hilfe der Argumentation aus Abschnitt 7.3 folgt, daß die Dimension der Räume $\Phi_{\bar{\gamma}}$ beliebig groß sein müßte, da jedes unabhängige Feld mit einem linear unabhängigen Anteil $\propto(E r)^{\frac{s-1}{2}} \mathrm{zu}$ den unteren Schranken beiträgt. Insofern ist das asymptotische Phasenraumkriterium im Bereich kleiner Skalen sensitiver als die bekannten Nuklearitäts- oder Kompaktheitsbedingungen.

Fermionen Unser Verfahren zur Konstruktion von Quantenfeldern haben wir bisher ausschließlich für bosonische Felder formuliert: Wir betrachten nur Größen, die sich als Grenzwerte lokaler Observablen darstellen lassen; nicht-observable Fermifelder können im berechneten Feldinhalt also nicht auftreten. Allerdings sollten dort observable Funktionen dieser Felder sichtbar sein, etwa $: \overline{\psi(x)} \gamma^{\mu} \psi(x)$ : im Fall eines Dirac-Feldes $\psi(x)$.

Um fermionische Modelle direkt zu analysieren, kann man den Formalismus jedoch abändern und statt der Observablenalgebren $\mathfrak{A}(\mathcal{O})$ die Feldalgebren $\mathfrak{F}(\mathcal{O})$ betrachten [BR81, sect. 5.4.3]. Die Vertauschungsrelationen der so erhaltenen Punktfelder, die ja bisher asymptotisch aus den Vertauschungsrelationen in den $\mathfrak{A}(\mathcal{O})$ gewonnen wurden, würden dann automatisch auch Antikommutatorrelationen beinhalten. Außerdem müßte die Symmetrieeigenschaft der $\mathfrak{A}(\mathcal{O})$ unter $\mathfrak{L}$ entsprechend durch eine Darstellung der Überlagerungsgruppe $\mathrm{SL}(2, \mathbb{C})$ auf den $\mathfrak{F}(\mathcal{O})$ ersetzt werden; das sollte aber mit Hilfe der in Abschnitt 4.2 entwickelten Methoden kein Hindernis beim Nachweis der Wightman-Axiome darstellen. 
In derselben Weise kann das Verfahren auch auf bosonische, aber nicht observable Felder ausgedehnt werden, z.B. auf Eichfelder (entsprechende Phasenraumeigenschaften vorausgesetzt).

2+1-dimensionale Modelle Da Informationen über konkrete Modelle, insbesondere in der konstruktiven Quantenfeldtheorie, hauptsächlich in niedrigen Raum-Zeit-Dimensionen vorliegen, ist es wünschenswert, die Anwendbarkeit unseres Formalismus auch im Fall niederdimensionaler freier Feldtheorien zu untersuchen. Wir betrachten dabei zunächst die $2+1$-dimensionale Theorie, die z.B. im Hinblick auf das wechselwirkende $\phi_{3}^{4}$-Modell [G.J87, sect. 23.1] von Interesse ist.

Man bemerkt hier, daß im Fall des reellen skalaren Feldes - der nach obigen Überlegungen als generisch anzusehen ist - die für die Feldstruktur wesentlichen EinteilchenraumEigenschaften 7.1 und 7.17 auch in $s=2$ Raumdimensionen noch erfüllt sind.

Probleme bereitet nur die Nuklearität bei festem $E$ und $r$, wie sie in Eigenschaft 7.2 gefordert wird; hier divergieren die Abschätzungen für $s=2$. Man kann vermuten, daß es sich dabei um ein rein technisches Problem handelt, da die Entwicklung bei festem $E$ und $r$ eher den Charakter einer Hilfsaussage hat und zur eigentlichen asymptotischen Approximation der Abbildung $\Xi$ nicht beiträgt. Diese Schwierigkeiten konnten jedoch bisher nicht überwunden werden.

Um das Phasenraumkriterium hier exakt behandeln zu können, kann man allerdings den massiven Fall näher betrachten. Vergleicht man mit den Abschätzungen in Anhang 7.2.C, dann sieht man, daß man zwar für die Spurnorm der dort behandelten Operatoren $T^{+}$ keine „skaleninvariante“ Abschätzung der Form

$$
\left\|T^{+}\right\|_{1} \leq E^{-\frac{1}{2}}(E r)^{\alpha} \cdot \text { const. }
$$

mehr erhält, wohl aber eine Abschätzung mit einem „etwas schlechteren Hochenergieverhalten":

$$
\left\|T^{+}\right\|_{1} \leq E^{-\frac{1}{2}} E^{\alpha} r^{\alpha-\epsilon} \cdot \text { const. }
$$

Modifiziert man nun die Kurzabstandsanalyse derart, daß statt

$$
\sup _{E \cdot r \leq w}\|\Xi-\psi\|_{E, r}
$$

der Ausdruck

$$
\sup _{E^{1+\epsilon} \cdot r \leq w}\|\Xi-\psi\|_{E, r}
$$

für ein gewisses $\epsilon>0$ im Limes $w \rightarrow 0$ analysiert wird, dann ist das resultierende Phasenraumkriterium auch in der $2+1$-dimensionalen massiven Theorie noch erfüllt, allerdings nicht mehr im masselosen Fall. Die anschließende Konstruktion der Punktfelder verläuft analog auch mit dem modifizierten Kriterium.円

\footnotetext{
${ }^{1}$ Man bemerkt, daß eine Theorie, die das ursprüngliche asymptotische Phasenraumkriterium erfüllt, auch dem modifizierten Kriterium genügt, denn das Gebiet in der E-r-Ebene, über das sich das zu bildende Supremum erstreckt, wird durch die Modifikation verkleinert. Die Menge der konstruierten Punktfelder ist dann in beiden Fällen dieselbe, da sie mit dem in (3.4.1) unabhängig vom Kriterium definierten Raum $\Phi_{\mathrm{FH}}$ übereinstimmt.
} 
Es wirkt sicher unnatürlich, hier die Analyse auf „Skalierungsorbits“ $E r \leq w$ zugunsten einer nicht-skaleninvarianten Form $E^{1+\epsilon} r \leq w$ aufzugeben. Man mag dies als eine Konsequenz der unphysikalischen Eigenschaften der dreidimensionalen Theorie verstehen, die im masselosen Fall zumindest bei festem $E$ und $r$ ein singuläres Phasenraumverhalten zeigt.

1+1-dimensionale Modelle Noch singulärer sind die Phasenraumeigenschaften von Theorien in nur einer Raumdimension. Hier bereitet nicht nur die Nuklearität bei festem $E$ und $r$ Probleme, sondern auch die asymptotischen Abschätzungen für Eigenschaft 7.1 lassen sich nicht mehr etablieren: Neben technischen Schwierigkeiten (Integraldivergenzen) wäre auch die bei formal gleichen Ergebnissen zu erwartende Funktion $\vartheta^{+}$nicht mehr strikt positiv (man hat $\vartheta^{+}(\kappa)=|\kappa|+\frac{s-1}{2}$ ); infolgedessen erhielte man in der Reihenentwicklung (7.2.60) beliebig viele Terme zum Er-Verhalten $\gamma=0$, entsprechend den Wick-Potenzen $: \phi^{n}$ : des Feldes. Die Räume $\Phi_{\bar{\gamma}}$ sind „unendlichdimensional“; das asymptotische Phasenraumkriterium kann nicht erfüllt sein.

Einen eventuellen Ausweg bietet die masselose $1+1$-dimensionale Stromalgebra, wie sie von Fredenhagen und Jörß [F.J96] im Zusammenhang mit Punktfeldeigenschaften betrachtet wurde. Hier wird das lokale Netz nur von den Strömen eines freien masselosen Feldes generiert (z.B. durch Restriktion des Einteilchenraums). Auf diese Weise tritt das beschriebene Problem der Wickprodukte gleichen Kurzabstandsverhaltens nicht auf. Im Prinzip ist es in diesem Fall also denkbar, endlichdimensionale Räume $\Phi_{\bar{\gamma}}$ zu erhalten. Allerdings läßt sich die spezielle Konstruktion der Einteilchenraum-Funktionen $g_{\kappa}^{ \pm}, h_{\kappa}^{ \pm}$aus Abschnitt 7.2 nicht direkt übertragen, sondern müßte geeignet modifiziert werden; abgesehen davon bleibt auch das Problem der Nuklearität bei festem $E$ und $r$ bestehen.

Modelle mit trivialem Feldinhalt Das in dieser Arbeit formulierte Phasenraumkriterium garantiert nur die Wohldefiniertheit („Endlichkeit“) des Feldinhalts einer Theorie; es macht keine Aussagen über dessen Umfang oder gar darüber, ob die algebraische Theorie vollständig aus ihm rekonstruiert werden kann. So könnten die lokalen Algebren durchaus Observablen beschreiben, die in endlich ausgedehnten Gebieten lokalisiert und im Phasenraumlimes nicht sichtbar sind. Ein Modell, das dieses Verhalten exemplarisch zeigt, wurde von Lutz betrachtet [Lut.97]. Es basiert auf einer freien Theorie (genauer einem reellen skalaren masselosen Feld in zunächst $(s+1)+1$ Raum-Zeit-Dimensionen), bei der jedoch die Algebren $\mathfrak{A}(r)$ für kleine $r$ quasi „ausgedünnt" werden: Man hat ${ }^{2}$

$$
\mathcal{L}^{ \pm}(r)=\overline{\omega^{\mp \frac{1}{2}}\left(\frac{\partial^{2}}{\partial x_{s+1}^{2}}\right)^{n(r)} \mathcal{D}_{\mathbb{C}}\left(\mathcal{O}_{r}\right)}
$$

mit einer Funktion $n: \mathbb{R}^{+} \rightarrow \mathbb{N}$, die für $r \rightarrow 0$ beliebig wächst. Die Koordinate $x_{s+1}$ ist hier eine „Hilfsdimension“; das Netz wird dann auf $s$ Raumdimensionen eingeschränkt.

In diesem Modell brechen die unteren Abschätzungen aus Abschnitt 7.3 - mangels verfügbarer Funktionen $f_{j}^{ \pm}-$zusammen, d.h. eine Mindestdimension der $\Phi_{\bar{\gamma}}$ größer als 1 kann nicht sichergestellt werden. Tatsächlich sollte sich das Phasenraumkriterium wie folgt etablieren lassen: Durch partielle Integration in (7.2.9) sieht man, daß der Beitrag von $h_{\kappa}^{ \pm}$ in der Einteilchenraum-Entwicklung verschwindet, falls $\kappa_{s+1}<2 n(r)$. Somit entfällt außer

\footnotetext{
${ }^{2}$ Die Notation ist gegenüber [Lı1.97] leicht verändert und unseren Konventionen angepaßt.
} 
dem Anteil des Einsoperators jeder festgehaltene Term $\sigma_{\mu} \phi_{\mu}$ in der Reihenentwicklung für $\Xi_{E, r}$, wenn man $r$ genügend klein wählt; man erhält

$$
\delta(\Xi-(\Omega|\cdot| \Omega) \mathbf{1})=0 .
$$

Das asymptotische Phasenraumkriterium ist trivialerweise erfüllt; wir erhalten $\Phi_{\bar{\gamma}}=\mathbb{C} \mathbf{1}$ für alle $\bar{\gamma}>0$. Die Phasenraumstruktur dieses Netzes führt also zu einem trivialen Punktfeldinhalt der Theorie. Wie in [Lut.97] gezeigt, ist auch der Skalenlimes des Netzes trivial.

\subsection{Wechselwirkung, Eichtheorien}

Wie wir gesehen haben, lassen sich viele qualitative Aspekte des Punktfeldinhalts und des Phasenraumverhaltens durch Betrachtung freier Modelle diskutieren. Wünschenswert wäre allerdings auch eine direkte Analyse realistischer wechselwirkender Theorien. Da es bisher nicht gelungen ist, Quantenfeldtheorien in physikalischer Raumzeit rigoros zu konstruieren, ist eine „konkrete“ Analyse zum momentanen Zeitpunkt nicht möglich. Der entwickelte Formalismus versetzt uns jedoch in die Lage, Aussagen ohne Kenntnis spezieller Modelle für alle relativistischen Quantentheorien zu treffen, die das asymptotische Phasenraumkriterium erfüllen.

Das betrachtete Phasenraumkriterium wurde mit physikalisch plausiblen Annahmen über das Kurzabstandsverhalten der Theorie motiviert. Wie explizit gezeigt, ist es in der freien Feldtheorie erfüllt. Seine Formulierung verwendet aber keine speziellen „freien“ Begriffe wie Wickprodukt, Fockraum oder Teilchenzahl, sondern sie basiert auf einem qualitativen Bild des Verhaltens der Theorie bei kleinen Wirkungen. Insofern scheint es plausibel, daß das Kriterium in jedem Modell mit „sinnvollem Punktfeldinhalt“ erfüllt ist. Genauer erwartet man dies zumindest in asymptotisch freien Theorien, deren Kurzabstandsverhalten dem einer freien Theorie entsprechen sollte; allgemeiner wird man ein analoges Verhalten in jedem Modell mit Ultraviolett-Fixpunkt der Renormierungsgruppe vermuten.

Setzen wir das asymptotische Phasenraumkriterium als physikalisch sinnvolle Annahme voraus, dann ist automatisch garantiert, daß die Theorie einen wohldefinierten Punktfeldinhalt aus Wightman-Feldern besitzt, der in einer aufsteigenden Kette von endlichdimensionalen Räumen $\Phi_{\bar{\gamma}}$ vorliegt; der Parameter $\bar{\gamma}$ klassifiziert dabei das Kurzabstandsverhalten der Felder. Zwischen den Feldern existiert stets eine Operatorproduktentwicklung in einem mathematisch wohldefinierten Sinn, unabhängig von der Störungstheorie. Allerdings können wir über die Größe des Feldinhalts a priori keine Aussagen machen.

Aus den Erfahrungen der Störungstheorie heraus vermutet man in wechselwirkenden Theorien, daß sich die Struktur der Räume $\Phi_{\bar{\gamma}}$ folgendermaßen von der in freien Theorien unterscheidet: Die Entartung des Kurzabstandsverhaltens der Felder wird beim „Einschalten der Kopplung“ aufgehoben; die Funktion $\operatorname{dim} \Phi_{\bar{\gamma}}$ weist deutlich mehr Sprungstellen auf, und diese werden dann auch nicht mehr bei ganz- oder halbzahligen Werten liegen, sondern bei allgemeinen reellen Werten („anomale Dimensionen“).

Es ist im allgemeinen nicht klar, ob der von uns konstruierte Satz von Punktfeldern in dem Sinne „vollständig“ ist, daß man aus ihm das lokale Netz $\mathcal{O} \mapsto \mathfrak{A}(\mathcal{O})$ vollständig rekonstruieren kann - für ein reelles skalares freies Feld ist dies der Fall, aber das oben erwähnte Modell von Lutz [Lut.97] bildet ein Gegenbeispiel. Wir können zur Klassifikation folgendes Schema angeben, das hier nur skizziert werden soll: 
Der Feldinhalt $\Phi_{\mathrm{FH}}$ erzeugt, wie man mit Hilfe der in [DSW86] entwickelten Methoden sehen sollte, folgendermaßen ein lokales Netz: Ist $\mathcal{P}(\mathcal{O})$ die von den Feldern mit in $\mathcal{O}$ lokalisierten Testfunktionen erzeugte Polynomialalgebra, d.h.

$$
\mathcal{P}(\mathcal{O})=\left\{\phi_{1}\left(f_{1}\right) \cdot \ldots \cdot \phi_{n}\left(f_{n}\right) \mid \phi_{j} \in \Phi_{\mathrm{FH}}, \operatorname{supp} f_{j} \subset \mathcal{O}, n \in \mathbb{N}\right\},
$$

und $\mathcal{P}(\mathcal{O})^{w}$ die schwache Kommutante dieser Operatoren, also

$$
\mathcal{P}(\mathcal{O})^{w}=\left\{B \in \mathfrak{B}(\mathcal{H}) \mid\left(\xi \mid B \phi \xi^{\prime}\right)=\left(\xi \mid \phi B \xi^{\prime}\right) \forall \xi, \xi^{\prime} \in \mathcal{C}^{\infty}(\mathcal{H}), \phi \in \mathcal{P}(\mathcal{O})\right\},
$$

dann definiert

$$
\mathfrak{A}_{\mathrm{F}}(\mathcal{O}):=\left(\mathcal{P}(\mathcal{O})^{w}\right)^{\prime}
$$

ein neues lokales Netz, wobei man sich auf bestimmte Gebiete $\mathcal{O}$ beschränkt, nämlich Doppelkegel, Keilgebiete und deren kausale Komplemente. Offenbar gilt

$$
\mathfrak{A}(\mathcal{O})^{\prime} \subset \mathcal{P}(\mathcal{O})^{w} \quad \Rightarrow \quad \mathfrak{A}_{\mathrm{F}}(\mathcal{O}) \subset \mathfrak{A}(\mathcal{O}),
$$

wobei die Inklusion im allgemeinen echt sein kann. Es ist klar, daß das Netz $\mathfrak{A}_{\mathrm{F}}$ ebenfalls das asymptotische Phasenraumkriterium erfüllt. Die Felder sind auch zu den $\mathfrak{A}_{\mathrm{F}}(\mathcal{O})$ affiliiert (der Beweis zu Lemma 3.16 überträgt sich); durch die in Satz 3.17 etablierten Approximationseigenschaften wissen wir dann, daß das ,verkleinerte“ Netz zum selben Feldinhalt führt wie das ursprüngliche:

$$
\Phi_{\mathrm{FH}}\left(\mathfrak{A}_{\mathrm{F}}\right)=\Phi_{\mathrm{FH}}(\mathfrak{A}) .
$$

Das Netz $\mathfrak{A}_{\mathrm{F}}$ ist in diesem Sinne ein „Fixpunkt unserer Konstruktion“.

Wir können also in $\mathfrak{A}$ ein von Punktfeldern erzeugtes Unternetz $\mathfrak{A}_{F}$ finden. Ist die Menge $\Phi_{\mathrm{FH}}$ von Feldern zusätzlich irreduzibel, gilt nach dem Reeh-Schlieder-Theorem also

$$
\overline{\mathcal{P}(\mathcal{O}) \Omega}=\mathcal{H} \text { für alle offenen Gebiete } \mathcal{O},
$$

dann kann man mit den in [BW75, BW76] entwickelten Techniken zeigen, daß das Netz $\mathfrak{A}_{\mathrm{F}}$ für Doppelkegel und Keilgebiete die Dualitätsbedingung erfüllt:

$$
\mathfrak{A}_{\mathrm{F}}\left(\mathcal{O}^{c}\right)=\mathfrak{A}_{\mathrm{F}}(\mathcal{O})^{\prime}
$$

wobei $\mathcal{O}^{c}$ das kausale Komplement von $\mathcal{O}$ bezeichnet. Aus

$$
\mathfrak{A}_{\mathrm{F}}\left(\mathcal{O}^{c}\right) \subset \mathfrak{A}\left(\mathcal{O}^{c}\right)
$$

folgt dann

$$
\mathfrak{A}_{\mathrm{F}}(\mathcal{O})=\mathfrak{A}_{\mathrm{F}}(\mathcal{O})^{\prime \prime}=\mathfrak{A}_{\mathrm{F}}\left(\mathcal{O}^{c}\right)^{\prime} \supset \mathfrak{A}\left(\mathcal{O}^{c}\right)^{\prime} \supset \mathfrak{A}(\mathcal{O}) \quad \Rightarrow \quad \mathfrak{A}_{\mathrm{F}}(\mathcal{O})=\mathfrak{A}(\mathcal{O}) .
$$

Die Netze $\mathfrak{A}$ und $\mathfrak{A}_{\mathrm{F}}$ stimmen also überein.

Auch der umgekehrte Schluß läßt sich durchführen: Ist $\overline{\mathcal{P}(\mathcal{O}) \Omega}$ für ein $\mathcal{O}$ ein echter Teilraum $\mathcal{H}_{\mathrm{F}} \subsetneq \mathcal{H}$, dann lassen die Operatoren $B \in \mathfrak{A}_{\mathrm{F}}(\mathcal{O})$ das orthogonale Komplement von $\mathcal{H}_{\mathrm{F}}$ stabil. Dasselbe gilt dann für jedes beschränkte $\mathcal{O}$ und damit auch für die von den 
$\mathfrak{A}_{\mathrm{F}}(\mathcal{O})$ erzeugte Algebra $\mathfrak{A}_{\mathrm{F}}^{\text {lok }}$. Da wir aber vorausgesetzt hatten, daß $\mathfrak{A}^{\text {lok }} \Omega$ dicht in $\mathcal{H}$ ist, muß $\mathfrak{A}_{\mathrm{F}}^{\text {lok }} \neq \mathfrak{A}^{\text {lok }}$ gelten, also folgt $\mathfrak{A}_{\mathrm{F}}(\mathcal{O}) \subsetneq \mathfrak{A}(\mathcal{O})$ zumindest für gewisse $\mathcal{O}$.

(Diese Argumente sollten natürlich genauer ausgearbeitet werden; es sei aber darauf hingewiesen, daß die Situation in unserem Fall deutlich „besser" ist als in [BW75, BW76, DSW86] angenommen, da wir über schärfere (polynomiale) Energieschranken und nach den Ergebnissen aus Abschnitt 3.4 auch über genaue Informationen über die Approximation von Feldern durch beschränkte Operatoren verfügen.)

Wir haben damit also ein Kriterium zur Klassifikation von lokalen Netzen anhand ihres Punktfeldinhalts erhalten: Im Fall

$$
\overline{\mathcal{P}(\mathcal{O}) \Omega}=\mathcal{H}
$$

stimmen $\mathfrak{A}$ und $\mathfrak{A}_{\mathrm{F}}$ überein, d.h. die Theorie wird vollständig durch ihren Punktfeldinhalt bestimmt. Ist aber

$$
\overline{\mathcal{P}(\mathcal{O}) \Omega} \subsetneq \mathcal{H} \text { für ein } \mathcal{O}
$$

dann gilt $\mathfrak{A}_{\mathrm{F}} \subsetneq \mathfrak{A}$; damit sind nicht-punktartig lokalisierte Observablen zur Beschreibung der Theorie wesentlich, etwa Mandelstam-Strings oder Wilson-Loops. Dies bietet eine Möglichkeit, um z.B. Eichtheorien von Punktfeld-Theorien nur durch Betrachtung observabler Größen zu unterscheiden.

Das ursprüngliche Fernziel von Haag und Ojima [H(.96] war nicht nur eine Klassifikation von Modellen, sondern auch eine Definition von Theorien direkt im algebraischen Rahmen. Dieses scheint jetzt insofern nähergerückt, als die Begriffe der "heuristischen“ Feldtheorie, wie Punktfelder, Normalprodukte und Feldgleichungen, nun im algebraischen Rahmen zur Verfügung stehen. Dabei darf allerdings nicht vergessen werden, daß auch die in der vorliegenden Arbeit beschriebene Methode nur auf eine Klassifikation und Analyse gegebener Modelle hinzielt, auf eine Auszeichnung einer Klasse von physikalisch sinnvollen Theorien im allgemeinen Rahmen. Die globalen Strukturen der Theorie, insbesondere auch der Generator der Zeittranslationen, werden stets als gegeben vorausgesetzt. Eine tatsächliche „Definition einer Theorie“ würde aber die Rekonstruktion eines Netzes aus gegebenem Punktfeldinhalt und der dort vorhandenen Produktstruktur erfordern (eventuell unter Zuhilfenahme einer Form von „Eichprinzip“), ohne dabei die Symmetrietransformationen a priori zu kennen. Ob dieses möglich ist und inwieweit es einen sinnvollen Rahmen zur Definition von Modellen bietet, ist momentan nicht absehbar. 



\section{Notationskonventionen}

Maßeinheiten Die Längen-, Massen- und Energieskalen sind anhand der Konvention $\hbar=1, c=1$ gewählt.

Minkowskiraum-Notation Wir verwenden den Minkowskiraum $\mathcal{M}=\mathbb{R}^{s+1}$; die Lorentzmetrik $\eta^{\mu \nu}$ habe die Signatur $(+--\ldots-)$. Elemente $x, p \in \mathcal{M}$ werden als $\left(x^{0}, \vec{x}\right)$ bzw. $\left(p^{0}, \vec{p}\right)$ geschrieben. Das indefinite Skalarprodukt lautet $p x=p_{\mu} x^{\mu}=p^{0} x^{0}-\vec{p} \vec{x}, p^{2}=\left(p^{0}\right)^{2}-$ $\vec{p}^{2}$. Dagegen bezeichnet $\|p\|=\left(\left(p^{0}\right)^{2}+\vec{p}^{2}\right)^{\frac{1}{2}}$ die euklidische Norm. Differentialoperatoren sind häufig abgekürzt als

$$
\partial_{\mu}=\frac{\partial}{\partial x^{\mu}},
$$

wobei die Ableitung $\partial_{0}$ nach der Zeitkoordinate auch als $\partial_{t}$ notiert wird.

Fouriertransformation Die Fouriertransformation schreiben wir mit folgender Vorzeichenkonvention:

$$
\begin{aligned}
\mathcal{F}: L^{2}\left(\mathbb{R}^{s}, d^{s} x\right) & \rightarrow L^{2}\left(\mathbb{R}^{s}, d^{s} p\right) ; & f(\vec{x}) & \mapsto \tilde{f}(\vec{p})=(2 \pi)^{-s / 2} \int e^{-i \vec{p} \vec{x}} f(\vec{x}) d^{s} x \\
\mathcal{F}^{-1}: L^{2}\left(\mathbb{R}^{s}, d^{s} p\right) & \rightarrow L^{2}\left(\mathbb{R}^{s}, d^{s} x\right) ; & \tilde{f}(\vec{p}) & \mapsto f(\vec{x})=(2 \pi)^{-s / 2} \int e^{+i \vec{p} \vec{x}} \tilde{f}(\vec{p}) d^{s} p
\end{aligned}
$$

Um die Notation nicht zu überfrachten, ist im Text die Tilde über der Fouriertransformierten nur selten notiert; manchmal bezeichnet sie auch die Rücktransformierte. Aus dem Zusammenhang und besonders aus der Bezeichnung des Funktionsarguments sollte stets klar sein, ob Orts- oder Impulsraum gemeint ist.

Kommutative Diagramme In Diagrammen von Abbildungen verwenden wir folgende Konventionen für die Form der Pfeile:

$\longrightarrow$ steht für eine allgemeine Abbildung,

$\hookrightarrow$ steht für eine Inklusionsabbildung (allgemeiner eine injektive Abbildung),

$\longleftrightarrow$ steht für eine surjektive Abbildung (Projektion),

$\longleftrightarrow$ steht für eine Bijektion,

......> steht für eine „zurückgezogene Abbildung“ (Wahl eines Urbilds),

$\longmapsto \quad$ bezeichnet wie üblich die Abbildung einzelner Elemente,

- - verbindet Paare dualer Räume (keine Abbildung).

Kommutative Kästchen in solchen Diagrammen kennzeichnen wir mit dem Symbol \#. Kästchen, die dieses Symbol nicht enthalten, müssen nicht notwendig kommutativ sein. 
Multiindizes An diversen Stellen des Textes, hauptsächlich in Kapitel 7, verwenden wir Multiindex-Schreibweisen; sie sind in Anhang 7.2.A erläutert.

Asymptotisches Verhalten von Funktionen Es seien $f(x), g(x)$ zwei Funktionen auf $\mathbb{R}^{+}$(oder auf einer geeigneten Nullumgebung) und $\rho=\left(x_{n}\right)$ eine Nullfolge. Wir schreiben

$f \leq \frac{\rho}{\rho} g, \quad$ falls $f\left(x_{n}\right) \leq c \cdot g\left(x_{n}\right)$ mit einer Konstanten $c$;

$f \geq g, \quad$ falls $f\left(x_{n}\right) \geq c \cdot g\left(x_{n}\right)$ mit einer Konstanten $c$;

$f \underset{\rho}{\sim} g, \quad$ falls $f \underset{\rho}{\geq} g$ und $f \underset{\rho}{\leq} g$

$f \underset{\rho}{\ll} g$, falls $\frac{f\left(x_{n}\right)}{g\left(x_{n}\right)} \underset{n \rightarrow \infty}{\longrightarrow} 0 ;$

$f \underset{\rho}{\gg} g$, falls $\frac{g\left(x_{n}\right)}{f\left(x_{n}\right)} \underset{n \rightarrow \infty}{\longrightarrow} 0$.

Gelten diese Abschätzungen unabhängig von einer speziellen Folge, dann notieren wir $f \leq \underset{\text { as }}{\leq} g$ usw. Siehe dazu auch Anhang 2.D.

Symbole und Kurzschreibweisen Im folgenden sind die im Text häufig verwendeten Symbole und Bezeichnungen aufgelistet. Zahlen in Klammern verweisen auf Gleichungsnummern, solche ohne Klammern auf Abschnitte oder Unterabschnitte.

\begin{tabular}{|c|c|c|}
\hline Symbol & eschreibung & Referenz \\
\hline$a^{*}(f), a(f)$ & rzeugungs- bzw. Vernichtungsoperator auf $\mathcal{H}$ & 7.1 .2 \\
\hline $\mathfrak{A}(\mathcal{O})$ & lokale Algebra zum Gebiet $\mathcal{O}$ & \\
\hline $\mathfrak{A}(r)$ & lokale Algebra zum Doppelkegel $\mathcal{O}_{r}$ & 1.3 .1 \\
\hline $\mathfrak{A}^{\text {lok }}$ & lokale Algebra aller beschränkten Gebiete & 1.3. \\
\hline$\overline{\mathfrak{A}}$ & Präkogarbe der lokalen Algebren & $(2.3 .1), 2 . \mathrm{A}$ \\
\hline$\overline{\mathfrak{A}}_{*}$ & Prägarbe der lokalisierten Funktionale & (2.A.1) \\
\hline $\mathfrak{B}(\mathcal{H})$ & Raum der beschränkten linearen Operatoren auf $\mathcal{H}$ & 1.3 .1 \\
\hline $\operatorname{Bild}_{L}(\ldots)$ & Links-Bild einer bilinearen Abbildung & (2.B.7) \\
\hline $\operatorname{Bild}_{R}(\ldots)$ & Rechts-Bild einer bilinearen Abbildung & (2.B.7) \\
\hline$d(g)$ & Ausdehnung des Trägers von Testfunktionen $g$ & $(5.5 .2$ \\
\hline$d(z)$ & $z$ zum Rand von $\mathcal{M}_{\mathrm{DR}}^{n}$ & $(5.2 .13)$ \\
\hline$\hat{d}(z)$ & Abstand von $z$ zum Rand von $\mathcal{M}_{\mathrm{R}}^{n+1}$ & $(5.2 .27)$ \\
\hline$D_{\mu}$ & Differentialoperator auf $\bar{\Sigma}$ & $(4.4 .8)$ \\
\hline $\mathcal{D}$ & Definitionsbereich der Wightman-Felder & 1.3 .2 \\
\hline $\mathcal{D}_{\mathbb{C}}(r)$ & komplexwertige Testfunktionen, Träger in $|\vec{x}|<r$ & \\
\hline $\mathcal{D}_{\mathbb{R}}(\mathcal{O})$ & eellwertige Testfunktionen mit Träger in $\mathcal{O}$ & \\
\hline $\mathfrak{D}_{k}$ & Raum der Differentialoperatoren $k$-ter Ordnung & (4.4.18) \\
\hline
\end{tabular}




\begin{tabular}{|c|c|c|}
\hline $\mathcal{F}$ & Operator der Fouriertransformation & siehe oben \\
\hline$H$ & Energieoperator (Hamiltonoperator) auf $\mathcal{H}$ & 1.3 .1 \\
\hline $\mathcal{H}$ & Vakuum-Hilbertraum & 1.3 .1 \\
\hline $\mathcal{H}_{n}$ & $n$-Teilchen-Raum & $(\sqrt{7.1 .6})$ \\
\hline $\mathcal{H}^{0}$ & Raum der Vektoren endlicher Teilchenzahl & $(7.1 .8)$ \\
\hline$J$ & antiunitäre Involution auf auf $\mathcal{K}$ & 7.1 .1 \\
\hline $\mathcal{K}$ & Einteilchenraum & 7.1 .1 \\
\hline $\operatorname{Kern}_{L}(\ldots)$ & Links-Kern einer bilinearen Abbildung & (2.B.7) \\
\hline $\operatorname{Kern}_{R}(\ldots)$ & Rechts-Kern einer bilinearen Abbildung & (2.B.7) \\
\hline $\mathfrak{L}$ & Lorentzgruppe & $1.3 .1, \mathrm{Fn} . \overline{1}$ \\
\hline $\mathcal{L}(\mathcal{O}), \mathcal{L}^{ \pm}(\mathcal{O})$ & „lokale“ Unterräume von $\mathcal{K}$ & 7.1 .3 \\
\hline $\mathcal{L}(r), \mathcal{L}^{ \pm}(r)$ & $=\mathcal{L}^{( \pm)}\left(\mathcal{O}_{r}\right)$ & 7.1 .3 \\
\hline $\mathcal{M}$ & Minkowskiraum $\left(=\mathbb{R}^{s+1}\right)$ & 1.3 .1 \\
\hline $\mathcal{M}_{\mathrm{R}}^{n+1}$ & Menge raumartig getrennter Vektoren in $\mathcal{M}^{n+1}$ & $(5.2 .4)$ \\
\hline $\mathcal{M}_{\mathrm{DR}}^{n}$ & Differenzen raumartig getrennter Vektoren $\left(\subset \mathcal{M}^{n}\right)$ & (5.2.11) \\
\hline $\mathfrak{M}^{n}$ & Menge der $n$-stelligen Multiindizes & 7.2.A \\
\hline $\mathfrak{M}^{\infty}$ & Menge der Multiindizes beliebiger Stellenzahl & $7.2 . \mathrm{A}$ \\
\hline $\mathrm{N}[\cdot]$ & Normalproduktraum & 6.2 .9 \\
\hline $\mathbb{N}$ & $=\{1,2,3, \ldots\}$ & \\
\hline $\mathbb{N}_{0}$ & $=\{0,1,2, \ldots\}$ & \\
\hline $\mathcal{O}$ & offenes Gebiet im Minkowskiraum $\mathcal{M}=\mathbb{R}^{s+1}$ & \\
\hline $\mathcal{O}_{r}$ & Standard-Doppelkegel (Mittelpukt 0, Radius $r$ ) & $(1.3 .7)$ \\
\hline$P_{\mu}$ & Impulsoperatoren auf $\mathcal{H} ; P_{0}=H$ & 1.3 .1 \\
\hline$P(E)$ & Spektralprojektor des Hamiltonoperators $H$ & 3. \\
\hline$P_{\mathcal{L}}^{ \pm}(r)$ & Projektor auf $\mathcal{L}^{ \pm}(r)$ & 7.1 .3 \\
\hline $\mathfrak{P}$ & Poincaré-Gruppe & 1.3.1, Fn. 1 \\
\hline$Q(E)$ & Spektralprojektor von $\omega$ & 7.1 .1 \\
\hline$R$ & $=(1+H)^{-1}$ & $(3.3 .9)$ \\
\hline $\mathbb{R}^{+}$ & $=\{x \in \mathbb{R} \mid x>0\}$ & \\
\hline $\mathbb{R}_{0}^{+}$ & $=\{x \in \mathbb{R} \mid x \geq 0\}$ & \\
\hline$V_{+}, \bar{V}_{+}$ & offener bzw. abgeschlossener Vorwärtslichtkegel & 1.3 .1 \\
\hline$s$ & Zahl der räumlichen Dimensionen & \\
\hline $\mathcal{S}\left(\mathbb{R}^{n}\right)$ & Raum der Schwartz'schen Testfunktionen auf $\mathbb{R}^{n}$ & \\
\hline $\mathcal{S}_{\mathbb{R}}\left(\mathbb{R}^{n}\right)$ & reellwertige Funktionen in $\mathcal{S}\left(\mathbb{R}^{n}\right)$ & \\
\hline $\operatorname{Span}(\ldots)$ & lineare Hülle einer Menge von Vektoren & \\
\hline $\operatorname{supp} f$ & Träger einer Funktion $f$ & \\
\hline $\operatorname{Symm}(\ldots)$ & Symmetrisierung im Fockraum & 7.1 .2 \\
\hline $\operatorname{tr} A$ & Spur eines Operators $A$ & \\
\hline$U(x, \Lambda)$ & Darsteller der Poincarétransformationen auf $\mathcal{H}$ & $(1.3 .5)$ \\
\hline$U_{\mathcal{K}}(x, \Lambda)$ & Darsteller der Poincarétransformationen auf $\mathcal{K}$ & 7.1 .1 \\
\hline$W(f)$ & Weyloperator & (7.1.11) \\
\hline
\end{tabular}


$\alpha(x, \Lambda) \quad$ Darsteller der Poincarégruppe auf $\mathfrak{A}(\mathcal{O})$ bzw. $\mathfrak{B}(\mathcal{H})$

$\gamma(\cdot) \quad$ asymptotischer Exponent

(1.3.4)

$\delta(\cdot) \quad$ Pseudometrik auf $\bar{\Psi}$

$\Delta_{+}(z) \quad$ Green'sche Funktion des Klein-Gordon-Operators

$\eta^{\mu \nu} \quad$ Lorentzmetrik; $\left(\eta^{\mu \nu}\right)=\operatorname{diag}(1,-1, \ldots,-1)$

$\Lambda \quad$ Lorentztransformation, $\Lambda \in \mathfrak{L}$

$\Xi \quad$ Phasenraum-Abbildung: $\Xi(\sigma, A)=\sigma(A)$

$\Sigma \quad$ Raum der normalen Funktionale auf $\mathfrak{B}(\mathcal{H})$

$\Sigma(E) \quad=P(E) \Sigma P(E)$

$\Sigma_{\bar{\gamma}} \quad$ Zustandshalm

2.3.16, 2.D

(7.4.5)

$\bar{\Sigma} \quad$ Präkogarbe der energiebeschränkten Funktionale

$\bar{\Sigma}^{*} \quad$ Prägarbe der Linearformen auf $\bar{\Sigma}$

$\bar{\Psi} \quad$ Raum bilinearer stetiger Abbildungen auf $\bar{\Sigma} \times \overline{\mathfrak{A}}$

$\bar{\Psi}_{0} \quad$ Abbildungen aus $\bar{\Psi}$ von asymptotisch endlichem Rang

$\bar{\Psi}_{0}^{P} \quad$ Abbildungen aus $\bar{\Psi}_{0}$ mit polynom. Energieschranken

$\Phi_{\bar{\gamma}} \quad$ Feldhalm (Raum von Punktfeldern)

$\Phi_{\mathrm{FH}} \quad$ Fredenhagen-Hertel-Feldinhalt

$\omega \quad$ Energieoperator im Einteilchenraum $\mathcal{K}$

$\Omega \quad$ Vakuumvektor in $\mathcal{H}$

(2.3.2), 2.A

(2.A.1)

$2.3,2 . \mathrm{A}$

$2.3,2 . \mathrm{C}$

1.3 .1

1 Einsoperator, Identität

$\square \quad$ D'Alembert-Operator; $\square=\partial_{\mu} \partial^{\mu}=\partial_{0}^{2}-\sum_{j=1}^{s} \partial_{j}^{2}$

$\triangle \quad$ Laplace-Operator; $\Delta=\sum_{j=1}^{s} \partial_{j}^{2}$

$\langle\cdot \mid \cdot\rangle \quad$ Skalarprodukt in $\mathcal{K}$

7.1 .1

$(\cdot \mid \cdot) \quad$ Skalarprodukt in $\mathcal{H}$

1.3 .1

$[\cdot, \cdot] \quad$ Kommutator; $[A, B]=A B-B A$

$\|\cdot\| \quad$ (Supremums-)Norm eines Operators; allg.: Norm

$\|\cdot\|_{1} \quad$ Spurnorm eines Operators; $L_{1}$-Norm einer Funktion

$\|\cdot\|_{2} \quad$ Hilbert-Schmidt-Norm eines Operators

$\|\cdot\|_{E} \quad\|\phi\|_{E}=\| \phi\left\lceil\Sigma(E) \| \quad\left(\phi \in \bar{\Sigma}^{*}\right)\right.$

(2.D.24)

$\|\cdot\|_{r} \quad\|\sigma\|_{r}=\| \sigma\left\lceil\mathfrak{A}(r) \| \quad\left(\sigma \in \overline{\mathfrak{A}}_{*}\right)\right.$

$\|\cdot\|_{E, r} \quad\|\psi\|_{E, r}=\| \psi\lceil\Sigma(E), \mathfrak{A}(r) \| \quad(\psi \in \bar{\Psi})$

(2.3.11), (2.D.21)

$\bar{M}^{w} \quad$ schwacher Abschluß einer Menge $M \subset \mathfrak{B}(\mathcal{H})$

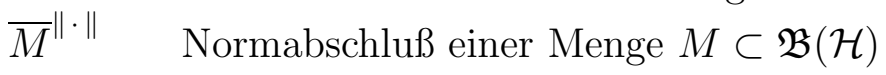

$\overrightarrow{\mathrm{R}} \quad$ raumartiger Limes

$\psi_{\mathrm{L}} \quad$ Linksadjungierte einer bilinearen Abbildung $\psi$

$\psi_{\mathrm{R}} \quad$ Rechtsadjungierte einer bilinearen Abbildung $\psi$

$\psi_{E, r} \quad$ Einschränkung eines $\psi \in \bar{\Psi}$ auf festes $E$ und $r$

$\mathfrak{B}_{1} \quad$ Einheitskugel eines Banachraums $\mathfrak{B}$

$\lceil\quad$ Einschränkung einer Abbildung

(2.3.7), (2.B.2)

(2.3.8), (2.B.4) 


\section{Literaturverzeichnis}

[Ara63] H. Araki: A Lattice of von Neumann Algebras Associated with the Quantum Theory of a Free Bose Field. J. Math. Phys. 4, 1343-1362 (1963).

[Bau75] K. Baumann: On Local Field Products in Special Wightman Theories. Commun. Math. Phys. 43, 73-87 (1975).

[BDF87] D. Buchholz, C. D'Antoni, K. Fredenhagen: The Universal Structure of Local Algebras. Commun. Math. Phys. 111, 123-135 (1987).

[BDH95] D. Brydges, J. Dimock, T. R. Hurd: The Short Distance Behaviour of $\left(\phi^{4}\right)_{3}$. Commun. Math. Phys. 172, 143-186 (1995).

[BDLR92] D. Buchholz, S. Doplicher, R. Longo, J. E. Roberts: A New Look at Goldstone's Theorem. Rev. Math. Phys. (Special Issue), 49-83 (1992).

[BDLW75] C. Bernard, A. Duncan, J. LoSecco, S. Weinberg: Exact spectral-function sum rules. Phys. Rev. D12(3), 792-804 (1975).

[BF77] D. Buchholz, K. Fredenhagen: Dilations and interactions. J. Math. Phys. 18, 1107-1111 (1977).

[BF87] D. Buchholz, K. Fredenhagen: Locality and the Structure of Particle States. Commun. Math. Phys. 85, 1-54 (1987).

[BJ86] D. Buchholz, P. Junglas: Local properties of equilibrum states and the particle spectrum in quantum field theory. Lett. Math. Phys. 11, 51-58 (1986).

[BJ87] D. Buchholz, P. Jacobi: On the Nuclearity Condition for Massless Fields. Lett. Math. Phys. 13, 313-323 (1987).

[BJ89] D. Buchholz, P. Junglas: On the Existence of Equilibrum States in Local Quantum Field Theory. Commun. Math. Phys. 121, 255-270 (1989).

[Boe55] H. Boerner: Darstellungen von Gruppen. Springer, Berlin, 1955.

[Bos98] H. Bostelmann: Zustandskeime in der lokalen Quantenfeldtheorie. Diplomarbeit, Universität Göttingen, Mai 1998.

[BP90] D. Buchholz, M. Porrmann: How small is the phase space in quantum field theory? Ann. Inst. H. Poincaré 52, 237-257 (1990). 
[BPS91] D. Buchholz, M. Porrmann, U. Stein: Dirac versus Wigner: Towards a universal particle concept in local quantum field theory. Phys. Lett. 267B, 377-381 (1991).

[BR79] O. Bratteli, D. W. Robinson: Operator Algebras and Quantum Statistical Mechanics, Band I. Springer, New York, 1979.

[BR81] O. Bratteli, D. W. Robinson: Operator Algebras and Quantum Statistical Mechanics, Band II. Springer, New York, 1981.

[Bra67] R. A. Brandt: Derivation of Renormalized Relativistic Perturbation Theory from Finite Local Field Equations. Annals of Physics 44, 221-265 (1967).

[Bra70] R. A. Brandt: Field Equations in Quantum Electrodynamics. Fortschr. Phys. 18, 249-283 (1970).

[Buc95] D. Buchholz: Unveröffentlichte Arbeit, 1995. Es handelt sich um die in [H()96] als Ref. 7 zitierten „unpublished notes“.

[Buc96] D. Buchholz: Phase space properties of local observables and structure of scaling limits. Ann. Inst. H. Poincaré 64, 433-459 (1996).

[BV95] D. Buchholz, R. Verch: Scaling Algebras and Renormalization Group in Algebraic Quantum Field Theory. Rev. Math. Phys. 7, 1195-1239 (1995).

[BW75] J. J. Bisognano, E. H. Wichmann: On the duality condition for a Hermitean scalar field. J. Math. Phys. 16, 985-1007 (1975).

[BW76] J. J. Bisognano, E. H. Wichmann: On the duality condition for quantum fields. J. Math. Phys. 17, 303-321 (1976).

[BW86] D. Buchholz, E. H. Wichmann: Causal Independence and the Energy-Level Density of States in Local Quantum Field Theory. Commun. Math. Phys. 106, 321-344 (1986).

[BW92] H. Baumgärtel, M. Wollenberg: Causal Nets of Operator Algebras. Akademie Verlag, Berlin, 1992.

[BY90] H.-J. Borchers, J. Yngvason: Positivity of Wightman Functionals and the Existence of Local Nets. Commun. Math. Phys. 127, 607-615 (1990).

[CG73] C. G. Callan, D. J. Gross: Bjorken Scaling in Quantum Field Theory. Phys. Rev. D8(12), 4383-4394 (1973).

[Cor84] J. F. Cornwell: Group Theory in Physics, Band II. Academic Press, London, 1984 .

[DF77] W. Driessler, J. Fröhlich: The reconstruction of local observable algebras from the Euclidean Green's functions of relativistic quantum field theory. Ann. Inst. H. Poincaré 27, 221-236 (1977). 
[DHR74] S. Doplicher, R. Haag, J. E. Roberts: Local Observables and Particle Statistics II. Commun. Math. Phys. 35, 49-85 (1974).

[DSW86] W. Driessler, S. J. Summers, E. H. Wichmann: On the Connection Between Quantum Fields and Von Neumann Algebras of Local Operators. Commun. Math. Phys. 105, 49-84 (1986).

[Fed61] P. G. Federbush: Operator Equations in Two Field Theory Models. Progr. Theoret. Phys. 26, 148-156 (1961).

[FH81] K. Fredenhagen, J. Hertel: Local Algebras of Observables and Pointlike Localized Fields. Commun. Math. Phys. 80, 555-561 (1981).

[FJ96] K. Fredenhagen, M. Jörß: Conformal Haag-Kastler Nets, Pointlike Localized Fields and the Existence of Operator Product Expansions. Commun. Math. Phys. 176, 541-554 (1996).

[FL94] W. Fischer, I. Lieb: Funktionentheorie. Vieweg, Braunschweig, 7. Auflage, 1994.

[GJ87] J. Glimm, A. Jaffe: Quantum Physics - A functional integral point of view. Springer, New York, second edition, 1987.

[Haa93] R. Haag: Local Quantum Physics and Models. Commun. Math. Phys. 155, 199-204 (1993).

[Haa96] R. Haag: Local Quantum Physics. Springer, Berlin, 2nd edition, 1996.

[HK64] R. Haag, D. Kastler: An algebraic approach to quantum field theory. J. Math. Phys. 5, 848-861 (1964).

[HO96] R. Haag, I. Ojima: On the problem of defining a specific theory within the frame of local quantum physics. Ann. Inst. H. Poincaré 64, 385-393 (1996).

[HS65] R. Haag, J. A. Swieca: When Does a Quantum Field Theory Describe Particles? Commun. Math. Phys. 1, 308-320 (1965).

[Jaf67] A. M. Jaffe: High-Energy Behaviour in Quantum Field Theory. I. Strictly Localizable Fields. Phys. Rev. 158(5), 1454-1461 (1967).

[Joh61] K. Johnson: Solution of the Equations for the Green's Functions of a two Dimensional Relativistic Field Theory. Nuovo Cimento 20, 773-790 (1961).

[Jos65] R. Jost: The General Theory of Quantized Fields. American Mathematical Society, Providence (Rhode Island), 1965.

[Jör96] M. Jörß: The Construction of Pointlike Localized Charged Fields from Conformal Haag-Kastler Nets. Lett. Math. Phys. 38, 257-274 (1996).

[Kat84] T. Kato: Perturbation Theory for Linear Operators. Springer, New York, 2nd corrected printing of the second edition, 1984. 
[KK92] G. Keller, C. Kopper: Perturbative Renormalization of Composite Field Operators via Flow Equations I. Commun. Math. Phys. 148, 445-467 (1992).

[KK93] G. Keller, C. Kopper: Perturbative Renormalization of Composite Field Operators via Flow Equations II: Short Distance Expansion. Commun. Math. Phys. 153, 245-276 (1993).

[Kos84] H. Kosaki: On the Continuity of the $\operatorname{Map} \varphi \rightarrow|\varphi|$ from the Predual of $a W^{*}$ Algebra. J. Funct. Anal. 59, 123-131 (1984). Hier: Lemma 3, p. 125.

[Low70] J. H. Lowenstein: Normal Products in the Thirring Model. Commun. Math. Phys. 16, 265-289 (1970).

[Low71] J. H. Lowenstein: Normal-Product Quantization of Currents in Lagrangian Field Theory. Phys. Rev. D4(8), 2281-2290 (1971).

[LS71] J. H. Lowenstein, B. Schroer: Locally Generated Dilation Charge in the Thirring Model. Phys. Rev. D3(8), 1981-1988 (1971).

[Lüs76] M. Lüscher: Operator Product Expansions on the Vacuum in Conformal Quantum Field Theory in Two Spacetime Dimensions. Commun. Math. Phys. 50, 23-52 (1976).

[Lut97] M. Lutz: Ein lokales Netz ohne Ultraviolettfixpunkte der Renormierungsgruppe. Diplomarbeit, Universität Hamburg, 1997.

[Mac77] G. Mack: Convergence of Operator Product Expansions on the Vacuum in Conformal Invariant Quantum Field Theory. Commun. Math. Phys. 53, 155-184 (1977).

[OZ72] P. Otterson, W. Zimmermann: On the Directional Dependence of Composite Field Operators. Commun. Math. Phys. 24, 107-132 (1972).

[Por99] M. Porrmann: The Concept of Particle Weights in Local Quantum Field Theory. Dissertation, Universität Göttingen, 1999.

[Rob62] D. W. Robinson: Support of a field in momentum space. Helv. Phys. Acta 35, 403-413 (1962).

[RS75] M. Reed, B. Simon: Methods of Modern Mathematical Physics, Band II: Fourier Analysis, Self-Adjointness. Academic Press, San Diego, 1975.

[RW86] J. Rehberg, M. Wollenberg: Quantum fields as pointlike localized objects. Math. Nachr. 125, 259-274 (1986).

[Som63] C. M. Sommerfield: On the Definition of Currents and the Action Principle in Field Theories of One Spatial Dimension. Annals of Physics 26, 1-43 (1963).

[SSe73] S. Schlieder, E. Seiler: Remarks Concerning the Connection between Properties of the 4-Point-Function and the Wilson-Zimmermann Expansion. Commun. Math. Phys. 31, 137-159 (1973). 
[SSV75] B. Schroer, J. A. Swieca, A. H. Völkel: Global operator expansions in conformally invariant relativistic quantum field theory. Phys. Rev. D11(6), 1509-1520 (1975).

[Sum87] S. J. Summers: From algebras of local observables to quantum fields: generalized $H$ bounds. Helv. Phys. Acta 60, 1004-1023 (1987).

[SW64] R. F. Streater, A. S. Wightman: PCT, Spin and Statistics, and All That. Benjamin, New York, 1964.

[Val54] J. G. Valatin: On the propagation functions of quantum electrodynamics. Proc. Roy. Soc. London A225, 535-548 (1954).

[Wei96] S. Weinberg: The Quantum Theory of Fields, Band II: Modern Applications. Cambridge University Press, 1996.

[WG65] A. Wightman, L. Gårding: Field as operator-valued distributions in relativistic quantum theory. Arkiv Fys. 28, 129-184 (1965).

[Wil69] K. G. Wilson: Non-Lagrangian Models of Current Algebra. Phys. Rev. 179(5), 1499-1512 (1969).

[Wil70a] K. G. Wilson: Anomalous Dimensions and the Breakdown of Scale Invariance in Perturbation Theory. Phys. Rev. D2(8), 1478-1493 (1970).

[Wil70b] K. G. Wilson: Operator-Product Expansions and Anomalous Dimensions in the Thirring Model. Phys. Rev. D2(8), 1473-1477 (1970).

[Wol85] M. Wollenberg: On the relation between quantum fields and local algebras of observables. Rep. Math. Phys. 22, 409-417 (1985).

[WZ72] K. G. Wilson, W. Zimmermann: Operator Product Expansions and Composite Field Operators in the General Framework of Quantum Field Theory. Commun. Math. Phys. 24, 87-106 (1972).

[Zim67] W. Zimmermann: Local Field Equations for $A^{4}$-Coupling in Renormalized Perturbation Theory. Commun. Math. Phys. 6, 161-188 (1967). Siehe auch die Ergänzung in Commun. Math. Phys. 10, 325-329 (1968).

[Zim70] W. Zimmermann: Local Operator Products and Renormalization in Quantum Field Theory. In: S. Deser, M. Grisaru, H. Pendleton (Herausgeber): Lectures on Elementary Particles and Quantum Field Theory, Band 1, Cambridge, 1970. MIT Press. 



\section{Danksagung}

Mein Dank gilt Prof. D. Buchholz für lange Diskussionen, sein kompromißloses Beharren auf Verbesserungen und dafür, daß er eine zügige Durchführung des Vorhabens möglich gemacht hat.

Für eine Diskussion, die am Beginn dieser Arbeit stand, geht mein Dank an Prof. R. Haag und Prof. I. Ojima.

Herr Prof. H.-J. Borchers (em.) hat sich kurzfristig bereit erklärt, das Korreferat der Dissertation zu übernehmen, wofür ich ihm sehr verbunden bin.

Die Arbeit wurde finanziell unterstützt im Rahmen des Promotionsschwerpunkts „Wechselwirkung“ des Evangelischen Studienwerks, Villigst, sowie durch Reisemittel aus den Stiftungen Dr. Berliner / Dr. Ungewitter an die Universität Göttingen. 



\title{
Lebenslauf
}

Henning Bostelmann, geboren am 19. Juli 1974 in Soltau

\author{
Mai 1993 Abitur am Gymnasium Soltau \\ Oktober 1993 Studium der Physik an der \\ bis Juli 1998 Georg-August-Universität Göttingen \\ Juni 1995 Diplomvorprüfung \\ Juli 1998 Diplomprüfung; Titel der Diplomarbeit: \\ "Zustandskeime in der lokalen Quantenfeldtheorie", \\ Anleitung: Prof. D. Buchholz \\ seit Doktorand am Institut für Theoretische Physik \\ Oktober 1998 der Universität Göttingen; \\ Betreuer der Dissertation: Prof. D. Buchholz
}

